Aus dem Institut für Sport und Sportwissenschaft (ISSW)

der Ruprecht-Karls-Universität Heidelberg

Geschäftsf. Leiter: Univ. Prof. Dr. Rüdiger Heim

\title{
Evaluation eines ambulanten psychoedukativen Bewegungsprogramms für Männer und Frauen mit Adipositas
}

an der Fakultät für Verhaltens- und Empirische Kulturwissenschaften zur Erlangung des akademischen Grades eines

Doktors der Philosophie (Dr. phil.)

genehmigte Dissertation

vorgelegt von

Jannis Alexandridis

aus Thessaloniki

Rimsting 2007 


\section{Erklärung}

Erklärung gemäß $\S 8$ Abs. 1 Buchst. b) der Promotionsordnung der Universität Heidelberg für die Fakultät für Verhaltens- und Empirische Kulturwissenschaften.

Ich erkläre, dass ich die vorgelegte Dissertation selbstständig angefertigt, nur die angegebenen Hilfsmittel benutzt und die Zitate gekennzeichnet habe.

Erklärung gemäß $\S 8$ Abs. 1 Buchst. c) der Promotionsordnung der Universität Heidelberg für die Fakultät für Verhaltens- und Empirische Kulturwissenschaften

Ich erkläre, dass ich die vorgelegte Dissertation in dieser oder einer anderen Form nicht anderweitig als Prüfungsarbeit verwendet oder einer anderen Fakultät als Dissertation vorgelegt habe.

Rimsting, im April 2007

Jannis Alexandridis 


\section{Vorwort und Danksagung}

An dem Zustandekommen der vorliegenden Arbeit war eine Reihe von Menschen beteiligt. Auch wenn ich an dieser Stelle nicht alle nennen kann, so gilt doch allen mein herzlicher Dank.

Die Daten für die vorliegende Untersuchung wurden neben meiner Tätigkeit als Sport- und Bewegungstherapeut in der Medizinisch-Psychosomatischen Klinik Roseneck in Prien am Chiemsee erhoben. Dem ärztlichen Leiter und Chefarzt, Herrn Prof. Dr. Manfred Fichter, und Chefarzt Dr. Ulrich Cuntz möchte ich an dieser Stelle für die guten wissenschaftlichen Rahmenbedingungen und die wertwollen Anregungen danken, die zum Gelingen meines Forschungsvorhabens beigetragen haben.

Mein besonderer Dank gilt der oberärztlichen Abteilungsleitung Dr. Christian Ehrig für die motivationale Unterstützung bei der Anfertigung meiner Arbeit.

Bei Prof. Dr. Gerhard Huber möchte ich mich für die engagierte und geduldige Betreuung bedanken.

Frau Dr. Katrin Wambach, Frau Dr. Silke Naab, Herrn Markus Fumi und Herrn Dr. Marian Cebulla gilt mein herzlicher Dank für die vielen inhaltlichen Diskussionen sowie nachhaltige aufmunternde und vorantreibende Unterstützung in den verschiedenen Phasen dieses Forschungsprojekts.

Danken möchte ich auch Herrn Dietrich Rötger, der das Manuskript Korrektur gelesen hat.

Dank gebührt der Firma Biospace für die Ausleihe des KörperzusammensetzungAnalysegeräts InBody 3.0.

Auch möchte ich allen Probanden(innen) Männer und Frauen, die als Freiwillige an der Untersuchung teilgenommen haben, für ihre Zeit und Mühe danken.

Der ganz besondere Dank gilt meiner Frau Katharina und meinen Kindern Konstantinos, Eleni und Anthea Zoi, die mir immer wieder Kraft gegeben haben. Jeder hat auf seine Weise dafür gesorgt, dass diese Dissertation entstehen konnte.

Rimsting, April 2007

Jannis Alexandridis 


\section{Inhaltsverzeichnis}

Tabellenverzeichnis

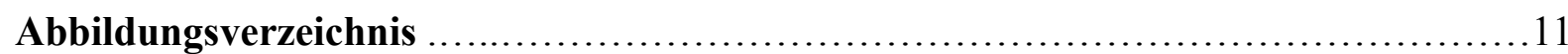

Abkürzungsverzeichnis ....................................................... 13

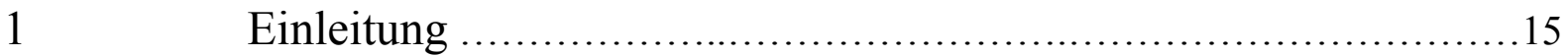

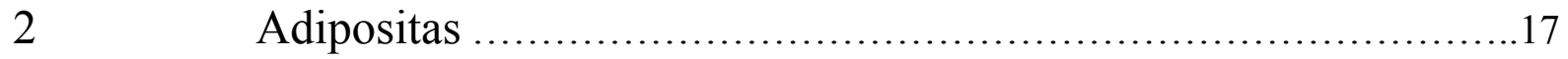

$2.1 \quad$ Bestimmung der Körperzusammensetzung ..............................19

2.1.1 Bioelektrische Impedanzanalyse (BIA) ................................21

$2.2 \quad$ Adipositas als Krankheit .................................................

Epidemiologie und Prävalenz der Adipositas .............................23

$2.4 \quad$ Sozioökonomische Faktoren der Adipositas ............................26

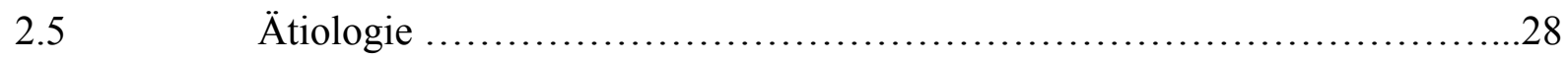

2.5.1 Körperliche Aktivität als Entstehungsursache der Adipositas ................33

2.5.2 Energieverbrauch und die Entstehung von Übergewicht .....................34

2.6 Medizinische Konsequenzen von Adipositas ...............................36

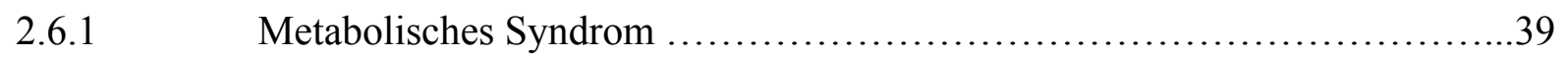

2.7 Psychosoziale Konsequenzen der Adipositas .............................40

2.7.1 Adipositas und gesundheitsbezogene Lebensqualität (HRQL) ...............42

$2.8 \quad$ Psychopathologische Aspekte ........................................45

2.8.1 Adipositas und Binge Eating Disorder (BED) ..........................46

2.9 Körperbild und Selbstkonzept bei Menschen mit Adipositas .................48

2.9.1 Körperbild und Motivation zur Gewichtsabnahme .........................54

2.9.2 Die Behandlung der Unzufriedenheit mit dem Körperbild bei Menschen mit Adipositas ............................................54

$3 \quad$ Therapieansätze bei Adipositas ........................................57

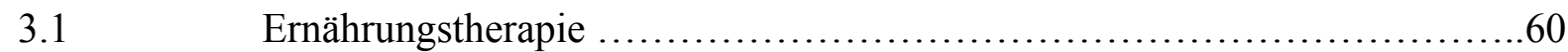

Medikamentöse Therapie .........................................62

Chirurgische Therapie ............................................64

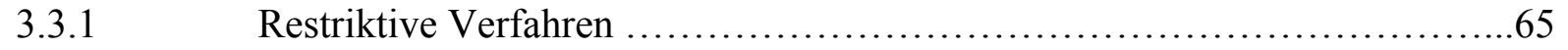

3.3.2 Magen-Bypass (Gastric-Bypass) und kombinierte Verfahren ................66

3.3.3 Plastisch-chirurgische Verfahren ......................................67

3.3.4 Zusammenfassende Bewertung chirurgischer Verfahren ...................67 


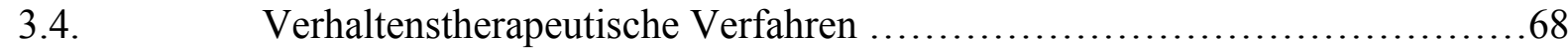

3.4.1 Prinzipien der Verhaltenstherapie bei Adipositas .........................71

3.4.2 Techniken der Verhaltenstherapie ................................... 73

3.4.3 Rahmenbedingungen der Verhaltenstherapie bei Adipositas ..................77

3.4.4 Verhaltenstherapeutische Techniken zur Bewegungssteigerung ..............78

4 Bewegungstherapie bei Adipositas ...............................83

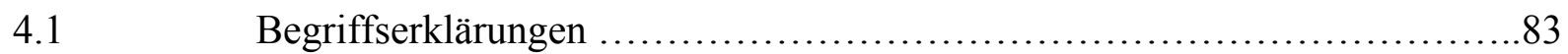

4.2 Bewegungsverhalten bei Adipositas .................................. 85

4.3 Bewegung als Ursache, Folge, Therapie und

4.4 Probleme bei der Erfassung von körperlicher

Aktivität bei Adipositas .................................................88

4.4.1 Kritik an den bestehenden Messverfahren ................................89

4.4.2 Methodische Probleme bei der Erfassung von körperlicher Aktivität ...........................................91

4.5 Bewegungstherapie und Gewichtskontrolle .............................992

4.6 Wirkungsmechanismen der körperlichen Aktivität bei der Gewichtskontrolle ............................................95

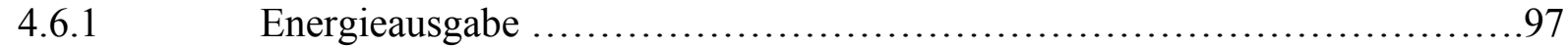

4.6.2 Appetit, Nahrungsaufnahme und Nahrungszusammensetzung ...............98

4.6.3 Körperzusammensetzung ............................................. 98

4.6.4 Grundumsatz ...................................................... 99

4.6.5 Psychologische Mechanismen ..................................... 100

4.6.5.1 Körperbild ....................................................... 102

4.6.5.2 Selbstwertgefühl und Selbstwirksamkeit ................................103

4.6.5.3 Bewältigungsstrategien ........................................... 105

4.6.5.4 Spekulativer Charakter der psychologischen Mechanismen ..................106

4.7 Weitere gesundheitliche Effekte der körperlichen

Aktivität bei Adipositas ...............................................107

4.7.1 Diabetes mellitus und Glykoseintoleranz .............................. 108

4.7.2 Verbesserungen des Lipidprofils .................................. 109

4.7.3 Abnahme von Blutdruck ............................................ 109

4.7.4 Verbesserung des Herz- Kreislaufsystems ............................109

$4.8 \quad$ Verordnung der körperlich-sportlichen Aktivität bei der

Behandlung von Adipositas 
4.9 Inhalte und Methoden des Trainings bei Adipositas .....................113

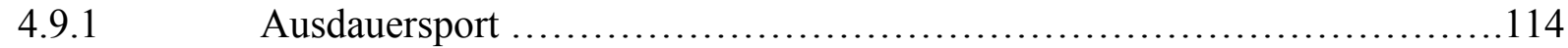

4.9.2 Krafttraining ...................................................... 115

4.9.3 „Lifestyle Physical Activity“ versus Bewegungsprogramme ................116

4.9.4 Heimtraining versus klinische Bewegungsprogramme ................... 120

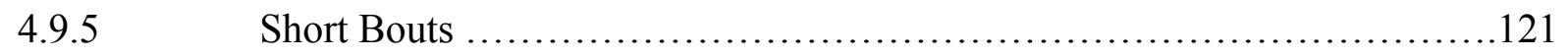

4.9.6 Die Individualisierung des Bewegungsprogramms .....................122

4.9.7 Zielsetzung eines Bewegungsprogramms .............................124

4.9.8 Die Bindung an das Bewegungsprogramm ............................ 125

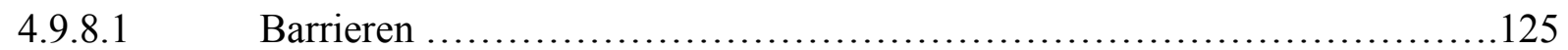

4.9.8.2 Rückfallprophylaxe und Bewältigungsstrategien ........................131

4.9.8.3 Verhaltenstherapeutische Strategien zur

Unterstützung der Bindung ........................................131

$5 \quad$ Methodik

5.1 Fragestellungen und Hypothesen der Untersuchung .

5.2 Die ambulanten Bewegungsprogramme als standardisierte achtwöchige Programme ............................................139

5.2.1 Methodik und Didaktik der Interventions- und Kontrollgruppe .............140

5.2.2 Zielsetzung des Treatments .........................................143

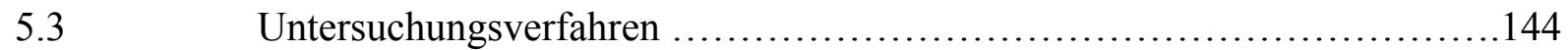

5.3.1 Fragebogen zu persönlichen Daten ................................. 145

5.3.2 Körpergewicht, Body Mass Index und Körperzusammensetzung ............145

5.3.3 Die Fahrradergometrische Belastungsuntersuchung .....................146

5.3.4 Fragebogen zum Gesundheitszustand (SF-36) .........................147

5.3.5 Das Frankfurter Körperkonzeptinventar/die Frankfurter

Körperkonzeptskalen FKKS nach Deusinger (1998) ......................148

5.3.6 Beck-Depressions-Inventar (BDI) .................................149

5.3.7 Das strukturierte Inventar für anorektische und bulimische

Essstörungen (SIAB) ................................................. 151

5.3.8 Der Dortmunder Fragebogen zur Bewegungstherapie .....................152

5.3.9 Fragebogen zur sportlichen und körperlichen Aktivität ...................152

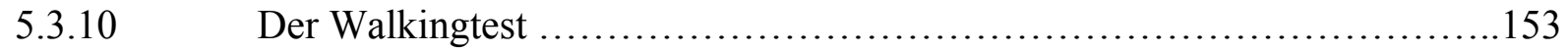

5.3.11 Der Bewegungskoordinationstest für die Praxis der Therapiekontrolle BKT-Kur nach Bös, Wydra und Karisch (1992) ..................155

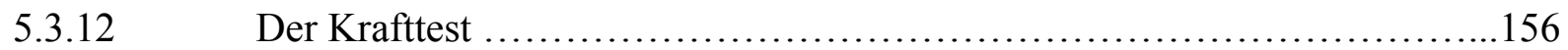




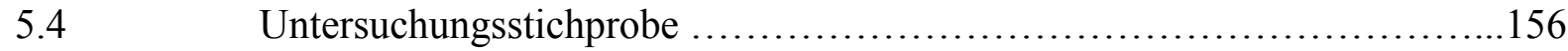

5.5 Methodische Überlegungen zu dem Untersuchungsdesign ..................157

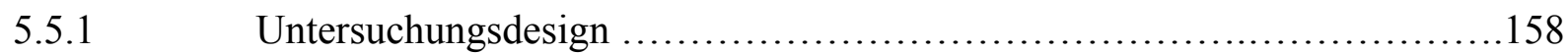

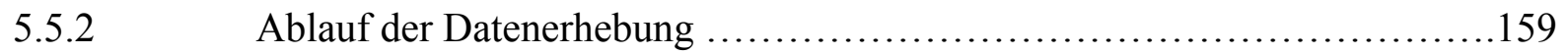

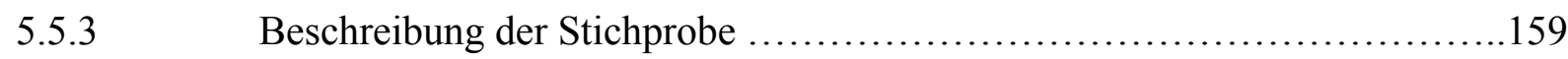

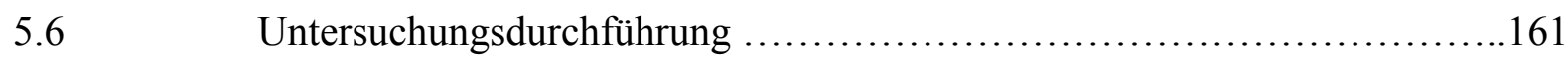

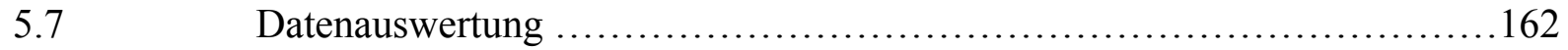

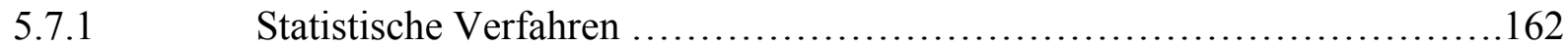

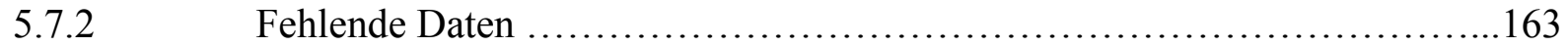

$6 \quad$ Untersuchungsergebnisse ..................................... 165

6.1 Körpergewicht, Body Mass Index und Körperzusammensetzung ….........165

6.2 Erfassung der gesundheitsbezogenen Lebensqualität .......................169

6.3 Erfassung des Körperkonzepts anhand

der Frankfurter Körperkonzeptskalen (FKKS) .............................176

6.4 Erfassung der Depressivität anhand

des Beck-Depressions-Inventars (BDI) ….............................. 183

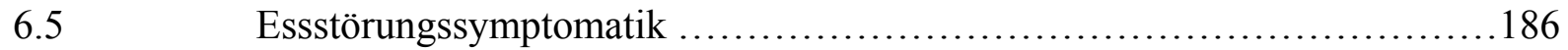

Wirkfaktoren des Behandlungsprogramms gemessen mit
dem Dortmunder Fragebogen zur Bewegungstherapie (DFBT) .............186

6.7 Die fahrradergometrische Belastungsuntersuchung ........................188

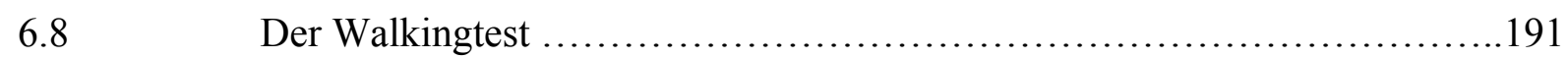

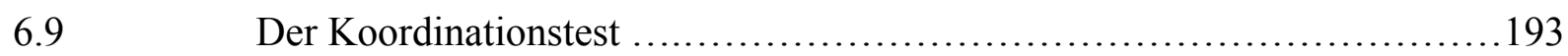

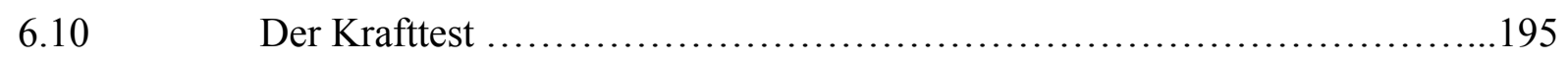

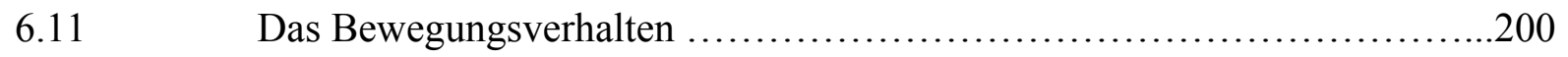

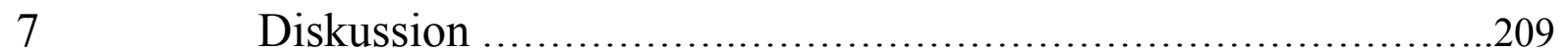

7.1 Körpergewicht, Body Mass Index und Körperzusammensetzung ...........209

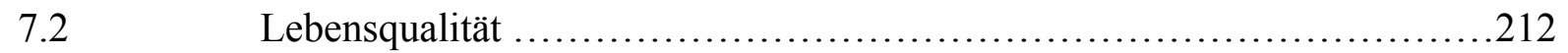

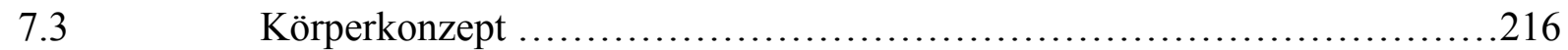

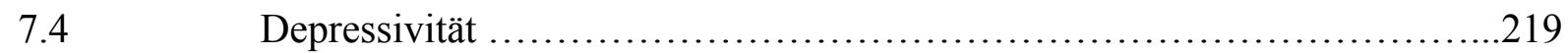

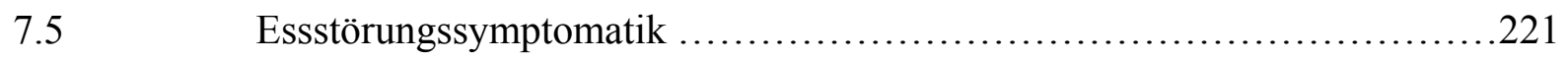

7.6 Subjektive Einschätzung der Wirkfaktoren der

Bewegungsprogramme durch die Teilnehmer ............................222

7.7 Allgemeine Ausdauerleistungsfähigkeit gemessen durch

fahrradergometrische Belastungsuntersuchung und Walkingtest .............224 


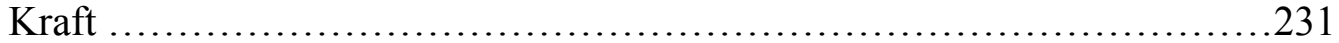




\section{Tabellenverzeichnis}

Tabelle 1: $\quad$ Gewichtsklassifikation bei Erwachsenen anhand des BMI (WHO, 2000, zitiert nach Deutsche Adipositas Gesellschaft, 2006, 6)

Tabelle 2: $\quad$ Labor- und Feldmethoden zur Bestimmung der

Körperzusammensetzung (Wagner, 2005, 9)

Tabelle 3: $\quad$ Prävalenz von Übergewicht und Adipositas in Deutschland (nach Ehrsam, 2004, 280)

Tabelle 4: $\quad$ Korrelate von Übergewicht und Adipositas oder Körpergewicht und Körperfettzunahme über die Zeit (Bouchard, 2000, 8)

Tabelle 5: $\quad$ Mit der Adipositas assoziierte Krankheiten (Wirth, 2003, 105) 38

Tabelle 6: $\quad$ Kriterien für die Diagnose des Metabolischen Syndroms nach AHA/NHLBI (Grundy et al., 2005, zitiert nach DAG, 2006, 9)

Tabelle 7: Interventionen und verhaltenstherapeutische Prinzipien in der Adipositasbehandlung (nach Munsch \& Margraf, 2002, 226) ....

Tabelle 8: Definition von körperlicher Aktivität und verwandter Begriffe nach dem Europäischen Informationszentrum für Lebensmittel, (EUFIC; European Food Information Council) (2006)

Tabelle 9: $\quad$ Zusammenfassung der Wirkungsmechanismen und ihre wissenschaftliche Evidenz (nach Baker und Brownell, 2000, 315)

Tabelle 10: Methoden und Inhalte von Experimental- und Kontrollgruppe

Tabelle 11: $\quad$ Subskalen und Itemanzahl des SF 36-Fragebogens (Bullinger \&

Kirchberger, 1998)

Tabelle 12: Die neun Skalen der Frankfurter Körperkonzeptskalen, die Kürzel und die zugehörigen Items (Deusinger, 1998)

Tabelle 13: Einteilung der depressiven Symptomatik nach Summenwert im BDI

(nach Hautzinger et al., 1995)

Tabelle 14: Normwerte für den Bewegungskoordinationstest $(\mathrm{N}=1831)$ nach

Bös et al., (1992) in Abhängigkeit von Geschlecht und Alter .155

Tabelle 15: Chronologie der Datenerhebung

Tabelle 16: Soziodemographische und anthropometrische Daten im

Gruppenvergleich

Tabelle 17: Zusätzliche Informationen, Medikation und Vorbehandlung

Tabelle 18: Mittelwerte und Standardabweichungen des Körpergewichts und

Body Mass Indexes der beiden Gruppen zu allen Messzeitpunkten, $\mathrm{t} 0=$ bei Aufnahme, $\mathrm{t} 1=4$ Wochen nach Aufnahme (Kursbeginn), t2 $=8$ Wochen nach Kursbeginn (Kursende), t3 $=6$ Monaten nach Kursende 
Tabelle 19: $\quad$ Signifikante Ergebnisse der zweifaktoriellen Varianzanalyse mit

Messwiederholungen für den $\mathrm{BMI}(\mathrm{N}=58)$....

Tabelle 20: $\quad$ Mittelwerte und Standardabweichungen der fettfreien

Körpermasse und des Körperfettanteils in kg der beiden Gruppen

zu allen Messzeitpunkten, $\mathrm{t} 0=$ bei Aufnahme, $\mathrm{t} 1=4$ Wochen nach

Aufnahme (Kursbeginn), t $2=8$ Wochen nach Kursbeginn

(Kursende), $\mathrm{t} 3=6$ Monate nach Kursende

Tabelle 21: $\quad$ Signifikante Ergebnisse der zweifaktoriellen Varianzanalyse mit

Messwiederholungen für den Körperfettanteil $(\mathrm{N}=58)$

Tabelle 22: Mittelwerte und Standardabweichungen der SF-36 Skalenwerte

der beiden Gruppen zu allen Messzeitpunkten, t $0=$ bei Aufnahme,

$\mathrm{t} 1=4$ Wochen nach Aufnahme (Kursbeginn), $\mathrm{t} 2=8$ Wochen nach

Kursbeginn (Kursende), t3 $=6$ Monate nach Kursende

Tabelle 23: $\quad$ Ergebnisse der zweifaktoriellen Varianzanalyse mit

Messwiederholungen für den SF-36 Skalenwert über 4

Messzeitpunkte

Tabelle 24: $\quad$ Mittelwerte und Standardabweichungen der körperlichen und psychischen Summenskalen für die deutsche Normstichprobe und beide Gruppen der Untersuchung zu allen Messzeitpunkten, $\mathrm{t} 0=$ bei Studieneinschluss, $\mathrm{t} 1=$ Kursbeginn, $\mathrm{t} 2=8$ Wochen nach Kursbeginn (Kursende), t3 $=6$ Monate nach Kursende

Tabelle 25: $\quad$ Signifikante Ergebnisse der zweifaktoriellen Varianzanalyse mit

Messwiederholungen für die körperlichen und psychischen

Summenskalenwerte über 4 Messzeitpunkte

Tabelle 26: $\quad$ Ergebnisse der Frankfurter Körperkonzeptskalen (FKKS) der

beiden Gruppen zu den vier Messzeitpunkten, $\mathrm{t} 0=$ bei

Studienbeginn, $\mathrm{t} 1=4$ Wochen nach Kursbeginn, $\mathrm{t} 2=8$ Wochen

nach Kursbeginn (Kursende), $\mathrm{t} 3=6$ Monate nach Kursende

Tabelle 27: $\quad$ Signifikante Ergebnisse der zweifaktoriellen Varianzanalyse mit

Messwiederholungen für die FKKS Skalenwerte $(\mathrm{N}=58)$

Tabelle 28: $\quad$ Ergebnisse des Beck-Depressions-Inventars (BDI) der beiden

Gruppen zu den vier Messzeitpunkten, $\mathrm{t} 0=$ bei Aufnahme, $\mathrm{t} 1=4$

Wochen nach Aufnahme (Kursbeginn), t $2=8$ Wochen nach

Kursbeginn (Kursende), t3 = 6 Monate nach Kursende und einer

gesunden Probandengruppe (Hautzinger et al., 1995)

Tabelle 29: $\quad$ Ergebnisse der zweifaktoriellen Varianzanalyse mit

Messwiederholungen für den BDI über die Messzeitpunkte

$\mathrm{t} 1-\mathrm{t} 2 / \mathrm{t} 2-\mathrm{t} 3,(\mathrm{~N}=58)$

Tabelle 30: Verlauf der BDI-Kategorien über die 4 Messzeitpunkte

Tabelle 31: Teilnehmer der Kontroll- und Experimentalgruppe, die die

Kriterien einer Binge Eating Disorder nach DSM - IV erfüllen

über 4 Messzeitpunkte 
Tabelle 32: Die 4 Skalen des DFBT mit ihren Mittelwerten,

Standardabweichungen für die Versuchsgruppe und für die

Kontrollgruppe zum Messzeitpunkt t2 (Ende des Treatments)

Tabelle 33: Subskala des DFBT „Erleben von Bewegung und Wohlbefinden“

im Gruppenvergleich bei Behandlungsende (t2)

Tabelle 34: $\quad$ Mittelwerte und Standardabweichungen der absoluten (L) und der gewichtsbezogenen (L/Gew) Leistungsfähigkeit bei einer Herzfrequenz von 130 Schlägen über 4 Messzeitpunkte $(\mathrm{t} 0=$ bei Aufnahme, $\mathrm{t} 1=4$ Wochen nach Aufnahme (Kursbeginn), $\mathrm{t} 2=8$

Wochen nach Kursbeginn (Kursende), t3 = 6 Monate nach

Kursende); $(\mathrm{N}=58)$

Tabelle 35: Die signifikanten Ergebnisse der zweifaktoriellen Varianzanalyse mit Messwiederholung auf dem Faktor Zeit ( $\mathrm{t} 0=$ bei Aufnahme, $\mathrm{t} 1=4$ Wochen nach Aufnahme (Kursbeginn), $\mathrm{t} 2=8$ Wochen nach Kursbeginn (Kursende), t3 $=6$ Monate nach Kursende) für die absolute (L) und gewichtsbezogene (L/Gew) Leistungsfähigkeit bei einer Herzfrequenz von 130 Schlägen; $(\mathrm{N}=58)$

Tabelle 36: Mittelwerte und Standardabweichung des Walkingtest-Index der beiden Gruppen zu den vier Messzeitpunkten, t0 = bei Aufnahme, $\mathrm{t} 1=4$ Wochen nach Aufnahme (Kursbeginn), $\mathrm{t} 2=8$ Wochen nach Kursbeginn (Kursende), t3 $=6$ Monate nach Kursende

Tabelle 37: $\quad$ Signifikante Ergebnisse der zweifaktoriellen Varianzanalyse mit Messwiederholungen auf dem Faktor Zeit (t0-t1; t1-t2, t2-t3) für den Walkingtest-Index

Tabelle 38: Klassifikation des Walkingtest-Index (WI) in 5 Stufen

(Bös et al., 1992, 173)

Tabelle 39: $\quad$ Ergebnisse des BKT-Kur der beiden Gruppen zu den vier

Messzeitpunkten, $\mathrm{t} 0=$ bei Aufnahme, $\mathrm{t} 1=4$ Wochen nach

Aufnahme (Kursbeginn), t $2=8$ Wochen nach Kursbeginn

(Kursende), $\mathrm{t} 3=6$ Monate nach Kursende

Tabelle 40: $\quad$ Signifikante Ergebnisse der zweifaktoriellen Varianzanalyse mit

Messwiederholungen der Koordinationsfähigkeit nach dem

BKT-Kur über 4 Messzeitpunkte

Tabelle 41: Normwerte für den Bewegungskoordinationstest, $\mathrm{N}=1831$,

(Bös et al., 1992, 160)

Tabelle 42: Ergebnisse der 3 Krafttestaufgaben der beiden Gruppen zu den vier Messzeitpunkten, $\mathrm{t} 0=$ bei Aufnahme, $\mathrm{t} 1=4$ Wochen nach Aufnahme (Kursbeginn), t $2=8$ Wochen nach Kursbeginn (Kursende), $\mathrm{t} 3=6$ Monate nach Kursende

Tabelle 43: $\quad$ Signifikante Ergebnisse der zweifaktoriellen Varianzanalyse mit Messwiederholungen der 3 Krafttestaufgaben über 4

Messzeitpunkte 
Tabelle 44: Relative und absolute Häufigkeit der sportlichen Aktivität der beiden Gruppen bei Aufnahme (t0) und 6 Monate nach Ende des

Programms (t3)

Tabelle 45: Relative und absolute Intensität der sportlichen Aktivität der

„Sportler" beider Gruppen bei Aufnahme (t0) und 6 Monate nach

Ende des Programms (t3)

Tabelle 46: Relative und absolute Häufigkeit der sportlichen Aktivität der

„Sportler“ beider Gruppen bei Aufnahme (t0) und 6 Monate nach

Ende des Programms (t3)

Tabelle 47: $\quad$ Relative und absolute Häufigkeit der Dauer der Übungseinheiten

der „Sportler“ beider Gruppen bei Aufnahme (t0) und 6 Monate

nach Ende des Programms (t3)

Tabelle 48: Relative und absolute Häufigkeit der Sportarten beider Gruppen

bei Aufnahme (t0) und 6 Monate nach Ende des Programms (t3);

$(\mathrm{N}=58)$

Tabelle 49: Relative und absolute Häufigkeit der Sportlertypen in beiden

Gruppen bei Aufnahme (t0) und 6 Monate nach Ende des

Programms (t3)

Tabelle 50: $\quad$ Relative und absolute Häufigkeit der habituellen sportlichen

Aktivität beider Gruppen bei Aufnahme (t0)

Tabelle 51: Relative und absolute Häufigkeit des Ausmaßes der sportlichen

Aktivität vor zehn Jahren (Teilnehmer, die vor 10 Jahren sportlich

aktiv waren) der beiden Gruppen bei Aufnahme (t0)

Tabelle 52: Relative und absolute Häufigkeit der habituellen Sportlertypen

beider Gruppen bei Aufnahme (t0)

Tabelle 53: Relative und absolute Häufigkeit der täglich zurückgelegten

Fußstrecke (werktags) der beiden Gruppen bei Aufnahme (t0)

und 6 Monate nach Ende des Programms (t3)

Tabelle 54: Relative und absolute Häufigkeit Außersportliche Benutzung des

Fahrrades(werktags) der beiden Gruppen bei Aufnahme (t0),

und 6 Monate nach Ende des Programms (t3)

Tabelle 55: Relative und absolute Häufigkeit der Teilnehmer beider Gruppen

die körperlich anstrengende Freizeittätigkeit ausführen

bei Aufnahme (t0) und 6 Monate nach Ende des Programms (t3) 


\section{Abbildungsverzeichnis}

Abbildung 1: Körperzusammensetzung nach dem 4-Kompartimentmodell

(Biospace, o.J.)

Abbildung 2: Biopsychosoziales Modell der Entstehung und Aufrechhaltung

von Übergewicht/Adipositas (Lehrke \& Laessle, 2003, 511)

Abbildung 3: Verschiedene Adipositaskausalitäten gruppiert nach

Verhalten, Stoffwechsel und biologischen

Einflussgrößen (Salbe \& Ravussin, 2000, 71)

Abbildung 4: Versuch einer Strukturierung des Gesamtkomplexes

Körpererfahrung (nach Bielefeld 1991, 17) ...

Abbildung 5: Elemente eines typischen Programms zur Verhaltensmodifikation

(nach Wirth, 2003, 132)

Abbildung 6: Vorgeschlagene Mechanismen und mögliche Pfadmodelle der

Verbindung körperliches Training und Gewichtskontrolle

(nach Baker und Brownell 2000, 315)

Abbildung 7: Wochenplan des psychoedukativen Bewegungsprogramms

Abbildung 8: Wochenplan des Fitnesstrainingsprogramms

Abbildung 9: Gewichtsbezogene Sollwerte der pulsbezogenen Leistungsfähigkeit

für Männer und Frauen (nach Rost, Heck \& Hollmann, o.J., 30)

Abbildung 10: Berechnungsformel des Walking-Index für Frauen und Männer

nach (Bös et al., 1992)

Abbildung 11: Graphische Darstellung des Untersuchungsdesigns

Abbildung 12: Verlauf des BMI der Experimental $(\mathrm{n}=30)$ und Kontrollgruppe $(\mathrm{n}=28)$ über 4 Messzeitpunkte im Gruppenvergleich

Abbildung 13: Verlauf der fettfreien Körpermasse und Fettmasse der

Experimentalgruppe $(\mathrm{n}=30)$ und Kontrollgruppe $(\mathrm{n}=28)$ anhand

der Mittelwerte über 4 Messzeitpunkte im Gruppenvergleich

Abbildung 14: Veränderungen der SF-36 Skalenwerte der Experimentalgruppe $(\mathrm{n}=30)$ und Kontrollgruppe $(\mathrm{n}=28)$ über 4 Messzeitpunkte im

Gruppenvergleich

Abbildung 15: Veränderungen der körperlichen und psychischen Summenskalen der Experimentalgruppe $(n=30)$ und Kontrollgruppe $(n=28)$ über 4 Messzeitpunkte im Vergleich mit der deutschen Normstichprobe

Abbildung 16: Veränderungen des Körperkonzepts (FKKS) der

Experimentalgruppe $(\mathrm{n}=30)$ und Kontrollgruppe $(\mathrm{n}=28)$

über 4 Messzeitpunkte im Gruppenvergleich

Abbildung 17: Ausrichtung des Körperkonzepts nach Deusinger

auf den einzelnen Subskalen 
Abbildung 18: Veränderungen der depressiven Symptomatik (BDI) der

Experimentalgruppe $(n=30)$ und Kontrollgruppe $(n=28)$ über 4

Messzeitpunkte im Gruppenvergleich

Abbildung 19: Verteilung auf die Kategorien im BDI der Experimentalgruppe

$(n=30)$ und Kontrollgruppe $(n=28)$ über 4 Messzeitpunkte im

Gruppenvergleich

Abbildung 20: Die 4 Subskalen des Dortmunder Fragebogens (DFBT) der

Experimentalgruppe $(n=29)$ und Kontrollgruppe $(n=28)$ zum

Messzeitpunkt t2 im Gruppenvergleich

Abbildung 21: Veränderungen der absoluten Leistungsfähigkeit (L) bei einer

Herzfrequenz von 130 Schlägen der Experimental- und

Kontrollgruppe über 4 Messzeitpunkte im Gruppenvergleich;

$(\mathrm{N}=58)$

Abbildung 22: Veränderungen der gewichtsbezogenen Leistungsfähigkeit (L/Gew)

bei einer Herzfrequenz von 130 Schlägen der Experimental- und

Kontrollgruppe über 4 Messzeitpunkte im Gruppen- und

Sollwertvergleich; $(\mathrm{N}=58)$

Abbildung 23: Verlauf des Walkingtest-Leistungsindex der Experimentalgruppe

$(n=29)$ und Kontrollgruppe $(n=25)$ über 4 Messzeitpunkte im

Gruppenvergleich

Abbildung 24: Verlauf der Koordinationsfähigkeit nach dem BKT-Kur der

Experimentalgruppe $(n=30)$ und Kontrollgruppe $(n=28)$ über 4

Messzeitpunkte im Gruppenvergleich

Abbildung 25: Verlauf der der Kraftaufgabe „Sit-ups“ der Experimentalgruppe

$(n=30)$ und Kontrollgruppe $(n=28)$ über 4 Messzeitpunkte im

Gruppenvergleich

Abbildung 26: Verlauf der Kraftaufgabe „Liegestütze“ der Experimentalgruppe

$(n=30)$ und Kontrollgruppe $(n=28)$ über 4 Messzeitpunkte im

Gruppenvergleich

Abbildung 27: Verlauf der Kraftaufgabe „Jump and reach“ der Experimentalgruppe

$(n=30)$ und Kontrollgruppe $(n=28)$ über 4 Messzeitpunkte im

Gruppenvergleich

Abbildung 28: Verhältnis von „Sportler“ und „Nicht-Sportler“ in der

Experimentalgruppe $(\mathrm{n}=30)$ und Kontrollgruppe $(\mathrm{n}=28)$ bei

Aufnahme (t0) und 6 Monate nach Ende des Programms (t3)

im Gruppenvergleich 


\section{$\underline{\text { Abkürzungsverzeichnis }}$}

$\alpha$ :

Cronbachs Alpha

ACSM: American College of Sports Medicine

AHA:

American Heart Association

AIT:

aktivitätsinduzierte Thermogenese

APA:

American Psychiatric Association

BED:

Binge Eating Disorders

BIA:

Bioelektrische Impedanzanalyse

BDI:

Beck Depressions Inventory

BKT-Kur:

Bewegungskoordinationstest - Kur

BMI:

Body Mass Index

CDC:

Centers for Disease Control and Prevention

DAG:

Deutsche Adipositas Gesellschaft

DFBT:

Dortmunder Fragebogen zur Bewegungstherapie

DFG:

Deutsche Gesellschaft für Ernährung

DSM:

Diagnostisches und Statistisches Manual psychischer Störungen

DVGS: Deutscher Verband für Gesundheitssport und Sporttherapie

EG: $\quad$ Experimentalgruppe

et al.: $\quad$ et altera (und andere)

FFM: fettfreie Masse

FKKS: $\quad$ Frankfurter Körperkonzeptskalen

HDL: High density lipoprotein

HRQL: health-related quality of life, Gesundheitsbezogene Lebensqualität

Hrsg: Herausgeber

IASO: International Association for the Study of Obesity

ICD: International Classification of Diseases

ITT: Intent to Treat

Kcal: Kilocalories

KG: Kontrollgruppe

LCD: $\quad$ Low-calorie -diets

LDL: $\quad$ Low density lipoprotein

MANOVA: Multiple Analysis of Variance

MET: $\quad$ Metabolische Einheit

NHLBI: $\quad$ National Heart, Lung and Blood Institute 


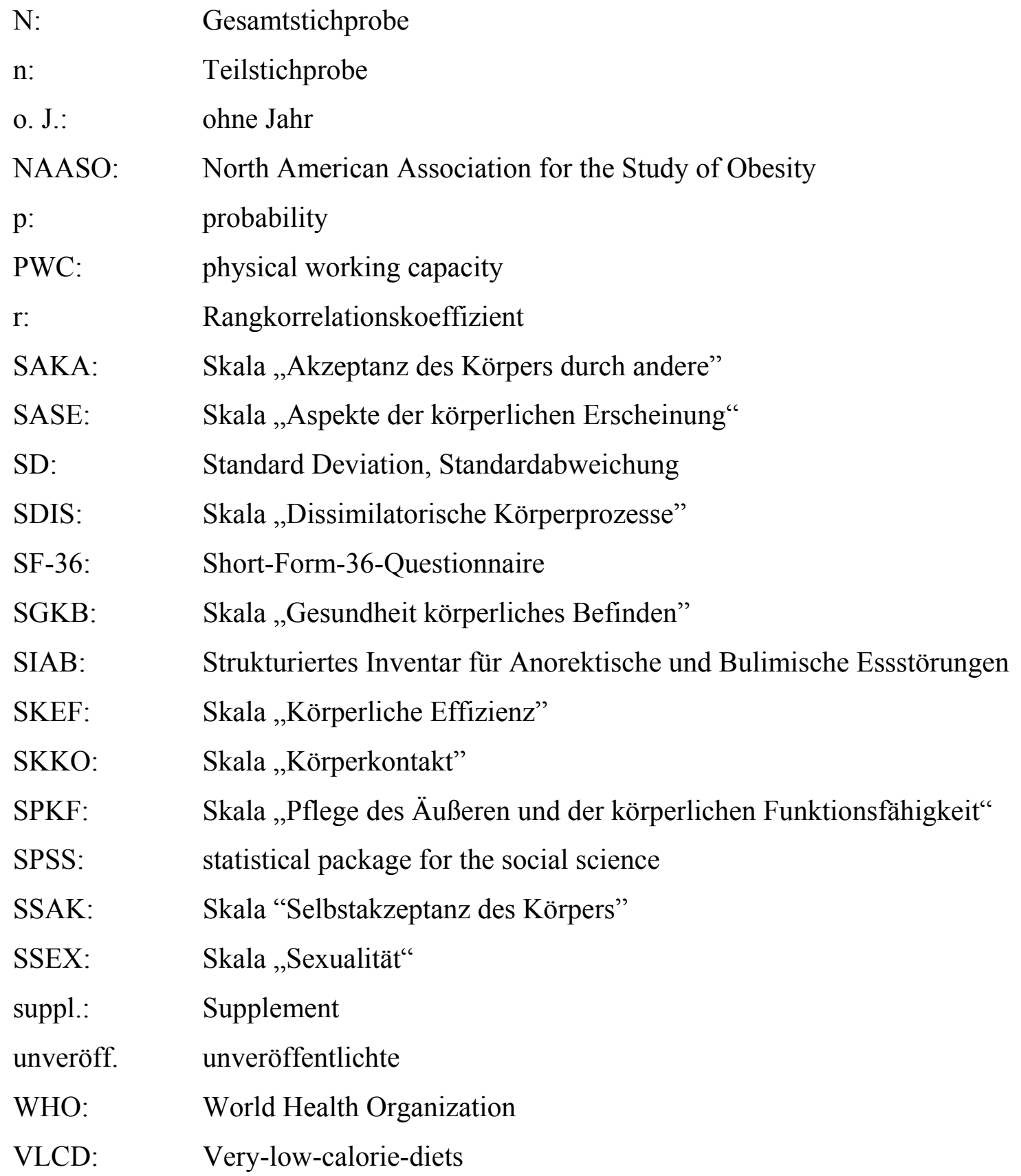




\section{Einleitung}

Epidemiologische Studien beschreiben eine auffallend hohe Prävalenz von Übergewicht und Adipositas sowohl bei Erwachsenen als auch bereits bei Kindern und Jugendlichen. Durch die mit der Adipositas verbundenen Folgekrankheiten ergeben sich immense Kosten durch Gesundheitsausgaben, Arbeitsunfälle und frühzeitige Berentung. Effektive und effiziente Programme für die Behandlung von Adipositas werden benötigt, um die Kosten und den Leidensdruck der Betroffenen zu mindern.

Das übergeordnete Ziel der Adipositastherapie ist die Gewichtsabnahme, um über eine Reduktion des Körpergewichts die Morbidität und Mortalität zu senken. Bewegungstherapeutische Maßnahmen sind feste Bestandteile der Adipositasbehandlung und sollen traditionell durch eine Erhöhung des Kalorienverbrauchs die Gewichtsreduktion unterstützen. Die Probleme einer solchen einseitigen Adipositasbehandlung sind hinreichend bekannt. Jo-Jo-Effekte, wie das im Englischen so bezeichnete „Weight Cycling“ folgen in den meisten Fällen einer anfänglichen Gewichtsreduktion.

Die Aufrechterhaltung eines bewegungsreichen Alltags bzw. sportlicher Aktivitäten ist daher von großer Bedeutung für das langfristige Gewichtsmanagement. Oft endet jedoch die körperliche Aktivität mit Beendigung von organisierten Bewegungsprogrammen. Eine langfristige Zunahme körperlicher Aktivität bei Menschen mit Adipositas ist nur schwer zu etablieren. In Anbetracht dieser Tatsache ist es notwendig, bestehende Bewegungsprogramme zu evaluieren, entsprechend zu modifizieren und gegebenenfalls neu zu entwickeln.

Neue Forschungsergebnisse geben hier richtungweisende Empfehlungen. Zum einen haben Untersuchungen gezeigt, dass die positiven Effekte bezogen auf Mortalität und Morbidität unabhängig von einer Gewichtsreduktion durch eine gesteigerte Fitness erreicht werden können. Zum anderen stellen psychosoziale Effekte wichtige Ressourcen bei der Verbesserung des Gesundheitsniveaus dar. Der Wirkung bewegungstherapeutischer Interventionen in Bezug auf psychologische Parameter wurde in der Adipositasforschung bisher wenig Aufmerksamkeit geschenkt (Blair \& Leermakers, 2004; Baker \& Brownell, 2000; Wing \& Jakicic, 2000).

Die vorliegende Untersuchung unternimmt, beeinflusst von einer psychosomatischmedizinischen Betrachtungsweise und der Verhaltenstherapie, einen Schritt, diese 
psychologische Wirkungsweise bewegungstherapeutischer Maßnahmen in den Vordergrund $\mathrm{zu}$ rücken. Vor dem Hintergrund langjähriger klinischer Erfahrung, verbunden mit einer bewegungswissenschaftlichen und psychotherapeutischen theoretischen Fundierung, wurde ein spezielles bewegungstherapeutisches Programm entwickelt. Dieses Programm bereitet sowohl geeignete Inhalte der Körper- und Bewegungserfahrung als auch verhaltenstherapeutische Verfahren den Bedürfnissen von Menschen mit Adipositas entsprechend methodisch auf und wird bezüglich seiner Wirksamkeit untersucht. So konnte eine spezifische standardisierte Behandlungsmethode entwickelt werden, die als solche einer wissenschaftlichen Evaluation zugänglich ist. In dieser randomisierten kontrollierten Studie sollen durch den Vergleich mit einem konventionellen Fitnesstrainingsprogramm die psychischen und die physischen Effekte von Bewegungstherapie bei Adipositas untersucht werden.

Im Theorieteil dieser bewegungswissenschaftlichen Arbeit wird zunächst das Krankheits- und Erscheinungsbild der Adipositas beschrieben. Die besondere Bedeutung der körperlich-sportlichen Aktivität und des Körperbildes werden in ihrer Relevanz für die Behandlung von Adipositas dargestellt. Es wird über die gängigen Behandlungsansätze im Sinne eines multimodalen Therapiekonzeptes berichtet, wobei die Verhaltenstherapie mit ihren Verfahren hier eine besondere Gewichtung erfährt. Die Bewegungstherapie wird zunächst ausgeklammert und anschließend in einem gesonderten Kapitel ausführlich dargestellt.

In der Literatur beschriebene bewegungstherapeutische Maßnahmen werden übersichtlich zusammengefasst und theoretisch eingeordnet. Auch in diesem Abschnitt werden die psychologischen Gesichtspunkte der sportlichen Aktivitäten und ihre mögliche Verknüpfung mit verhaltenstherapeutischen Verfahren in den Vordergrund gestellt. Wichtige Forschungsergebnisse aus dem Themenkreis des Sports und der Bewegungspsychotherapie bei Adipositas ergänzen das Kapitel. Im empirischen Teil werden die untersuchten ambulanten Bewegungsprogramme, die Stichprobe und die eingesetzten Untersuchungsverfahren beschrieben und anschließend diskutiert, um die therapeutische Relevanz des psychoedukativen Bewegungsprogramms im Rahmen eines Konzepts zur langfristig erfolgreichen Adipositastherapie einzuordnen. 


\section{Adipositas}

Die Begriffe „Übergewicht“, „Adipositas“, „Fettsucht“ und „Fettleibigkeit“ werden in der Fach- wie in der Laienliteratur häufig unklar voneinander abgegrenzt.

Fachlich von Bedeutung wäre vor allem die Unterscheidung von zwei verschiedenen Konzepten. Zum einen das Überschreiten des Standardlevels für die jeweilige Größe und das Alter, welches korrekter Weise mit Übergewicht bezeichnet werden sollte und nicht zwangsläufig einer Adipositas entsprechen muss.

Zum anderen die Adipositas, definiert als eine über das Normalmaß hinausgehende Vermehrung des Körperfettes.

Übergewicht kann auch durch Muskelhypertrophie wie bei aktiven Kraftsportlern entstehen. Aus dem Grund können Individuen und Athleten als übergewichtig, aber nicht adipös klassifiziert werden. Ist der menschliche Körper nicht extremen Trainingsreizen ausgesetzt, kann man davon ausgehen, dass das Mehr an Gewicht eine Adipositas ist.

Im medizinischen Sinn spricht man von Adipositas, wenn ein bestimmtes Ausmaß an Körpergewicht erreicht ist. Der Bereich des Adipösen wird dann in unterschiedliche Schweregrade eingeteilt. Berechnungsgrundlage für diese Klassifikation ist der Körpermassenindex [Body-Mass-Index (BMI)]. Der BMI ist der Quotient aus Gewicht und Körpergröße zum Quadrat.

$$
\mathrm{BMI}=\frac{\text { Körpergewicht in } \mathrm{kg}}{(\text { Körpergröße in } \mathrm{m})^{2}}
$$

Adipositas beginnt bei einem BMI $\geq 30 \mathrm{~kg} / \mathrm{m}^{2}$ und wird dann in Fünferschritten in drei Ausprägungsgrade unterteilt.

Die Gewichtsklassifikation bei Erwachsenen wird von der Weltgesundheitsorganisation (WHO) anhand des BMI in der folgenden Tabelle dargestellt: 
Tabelle 1: Gewichtsklassifikation bei Erwachsenen anhand des BMI (WHO, 2000, zitiert nach Deutsche Adipositas Gesellschaft, 2006, 6)

\begin{tabular}{|l|l|l|}
\hline Kategorie & BMI & $\begin{array}{l}\text { Risiko für Begleiterkrankungen } \\
\text { des Übergewichts }\end{array}$ \\
\hline Untergewicht & $<18.5$ & niedrig \\
\hline Normalgewicht & $18.5-24,9$ & durchschnittlich \\
\hline Übergewicht & $>25.0$ & durchschnittlich \\
Präadipositas & $25-29,9$ & gering erhöht \\
Adipositas Grad I & $30-34,9$ & erhöht \\
Adipositas Grad II & $35-39,9$ & hoch \\
Adipositas Grad III & $\geq 40$ & sehr hoch \\
\hline
\end{tabular}

Diese Klassifizierung auf der Grundlage des BMI wird besonders bei der Untersuchung großer Populationen in der Epidemiologie angewendet und dient einer guten Vorabeinteilung (Melanson, McInnis \& Rippe, 2003).

Der BMI gilt im Allgemeinen aufgrund seiner mathematischen Eigenschaften, der geringen Korrelation zur Körpergröße, hohen Korrelation zur Fettmasse und Einfachheit der Berechnung als valides Maß für die Schätzung der Fettmasse bei Erwachsenen und Kindern (Lehrke \& Laessle, 2003).

Der BMI ist nur eingeschränkt verwendbar bei Schwangeren oder Kindern im Wachstumsalter. Die Formel gibt keinen Aufschluss, über die Körperzusammensetzung. Sie unterscheidet nicht zwischen Muskel- und Fettgewebe und durch sie können Übergewichtige nicht von Adipösen unterschieden werden.

Deshalb können die durch den BMI errechneten Werte nur als Orientierung gelten, insbesondere für die sporttreibende Bevölkerung (Melanson et al., 2003). Eine falsche Klassifizierung könnte ein potentielles Problem bei nicht klinischen Settings sein (Field, Barnoya \& Colditz, 2004).

Zur genaueren Bestimmung von Adipositas empfiehlt sich daher neben einer relativen Gewichtsmessung auch die Bestimmung weiterer Kriterien, wie die des Körperfettanteils und dessen Verteilung, sowie die Berücksichtigung des Alters und Geschlechts (Lehrke \& Laessle, 2003; Björntorp, 2002). So können Fitness und Belastbarkeit für eine adäquate Therapie oder differenzierte Bewertung epidemiologischer Daten berücksichtigt werden. 


\subsection{Bestimmung der Körperzusammensetzung}

Die Körperzusammensetzung gilt als wesentlicher Parameter zur Bewertung des Gesundheits- und Ernährungszustandes. Das gewichtsabhängige Gesundheitsrisiko kann durch die Erfassung der Körperzusammensetzung differenziert werden (Müller, Mast, Bosy-Westphal, \& Danielzik, 2003; Bosy-Westphal et al., 2006).

Die Bestimmung der Körperzusammensetzung ist aus klinischer Sicht vorwiegend aus zwei Gründen notwendig. Erstens ist für die Entstehung kardiovaskulärer Risikofaktoren nicht das Körpergewicht, sondern die Körperfettmasse bzw. die Fettverteilung (viszerale Fettmasse) entscheidend (Deutsche Adipositas Gesellschaft, 2006). Zweitens spielt die Fettverteilung bezüglich der Morbidität eine wichtige Rolle (Wirth, 2000; Lohman, 2002).

Die Bestimmung weiterer Komponenten der Körperzusammensetzung, wie die der fettfreien Masse (FFM) und der Körperzellmasse (BCM, Body Cell Mass), ist für die Adipositasbehandlung genauso sinnvoll. Auf diesem Wege können die Effekte von Diäten (z.B. unerwünschter Verlust an Körperzellmasse bei drastischer Gewichtsreduktion) genau beurteilt werden oder auch die spezifische Wirkung eines Bewegungsprogramms (z.B. Zunahme von Körperzellmasse und fettfreier Masse bei gleichzeitiger Reduktion der Fettmasse) erfasst werden (Müller et al., 2003).

Die Erfassung der Körperzusammensetzung ist heute durch eine Vielzahl von Methoden möglich. Die folgende Tabelle fasst die gebräuchlichsten Labor- und Feldmethoden zusammen: 
Tabelle 2: Labor- und Feldmethoden zur Bestimmung der Körperzusammensetzung (Wagner, 2005, 9)

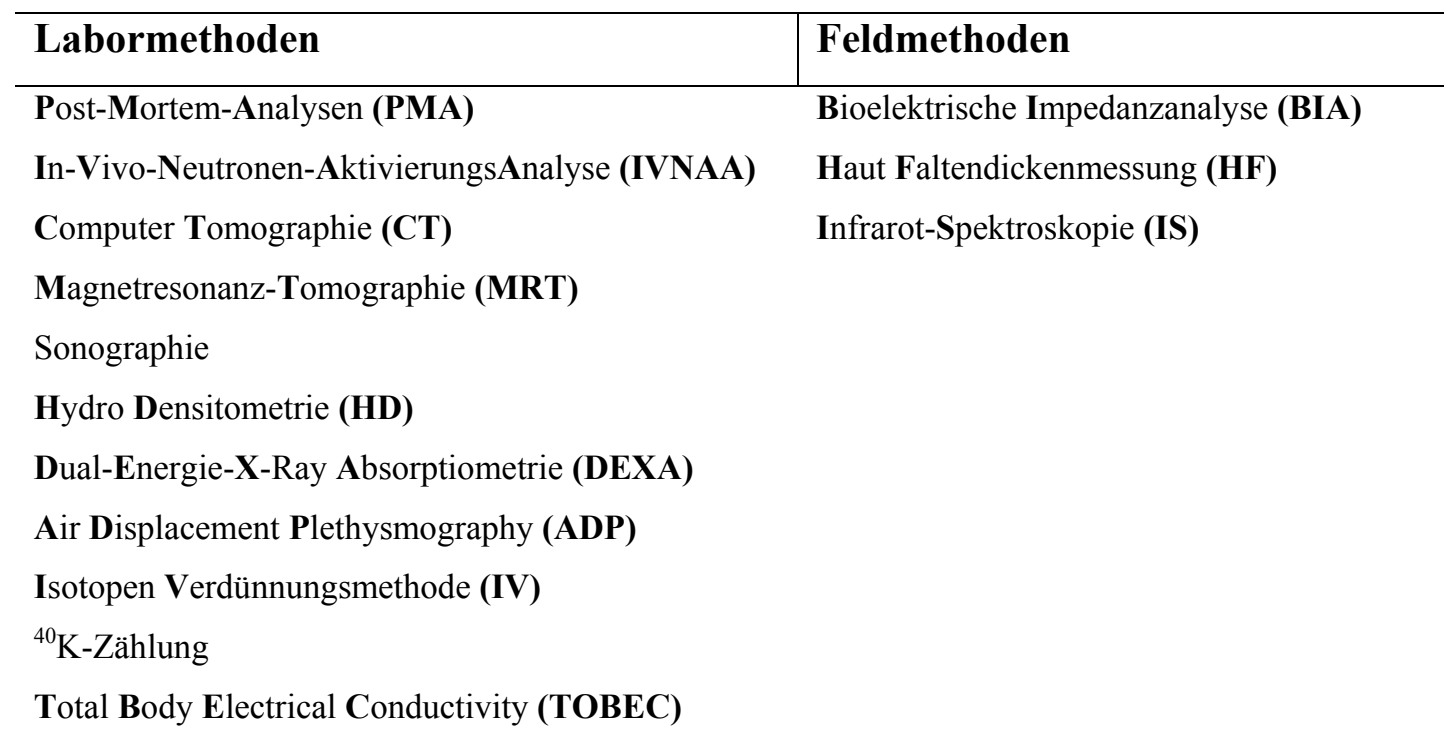

Einzelne dieser Methoden beziehen sich auf unterschiedliche Modelle der Körperzusammensetzung wie anatomische, biochemische, elementare und metabolische. Diese Modelle unterteilen den menschlichen Körper in Kompartimente. So entstehen 2-, 3-, oder 4-Kompartimentenmodelle.

Grundsätzlich werden für die Berechnung der Körperzusammensetzung physikalische Kenngrößen, wie z.B. Dichte oder Widerstand verschiedener Gewebe, gemessen. Mit Hilfe spezieller Regressionsgleichungen, die neben den Messwerten zusätzliche Parameter, wie z.B. Alter oder Geschlecht, berücksichtigen, können die einzelnen Kompartimente des Körpers berechnet werden.

Die Wahl der Methode zur Erfassung der Körperzusammensetzung hängt von der jeweiligen Zielgröße, aber auch von den Rahmenbedingungen ab. Routineerfassungen sollten einfach, schnell und preiswert sein. Weit verbreitet sind die Anthropometrie sowie die Bioimpedanzanalyse (BIA). Die anthropometrischen Messverfahren berücksichtigen Körperbaumaße und Hautfaltendicke (BosyWestphal et al., 2006). Aufwändigere und kostenintensivere Verfahren, wie die Neutronenaktivierung, werden vornehmlich im Rahmen wissenschaftlicher Untersuchungen angewandt (Wirth, 2000; Munsch, 2002).

Die Densitometrie, Isotopendilution und DEXA sind heute die allgemein akzeptierten Referenzmethoden (Goldstandards) für so genannte indirekte Methoden wie z. B. die Anthropometrie oder BIA (Müller et al., 2003; Lohman, 2002). Diese 
sind leicht durchzuführen, vereinfachen die Körperzusammensetzung aber stark. Durch die Kombination verschiedener Methoden können die Schwächen der einzelnen kompensiert werden.

$\mathrm{Da}$ in der vorliegenden Arbeit die BIA angewandt wurde, wird diese Methode im Folgenden theoretisch dargestellt.

\subsubsection{Bioelektrische Impedanzanalyse (BIA)}

Ein zuverlässiges Standardverfahren zur Bestimmung der Körperzusammensetzung ist die Bioelektrische Impedanzanalyse (BIA). Sie ermöglicht im Rahmen des 3Kompartimentenmodells eine Differenzierung zwischen intra- und extrazellulärer Flüssigkeit. Gemessen wird der Widerstand des Körpers gegen Wechselstrom. Dabei stellt die fettfreie Masse durch ihren Anteil an Wasser und Elektrolyten einen guten Leiter dar. Der Widerstand ist somit sehr gering. Die Fettmasse leitet den Strom hingegen nur sehr schlecht. Damit verbunden ist ein hoher elektrischer Widerstand.

Die BIA- Methode ist indirekt und nicht invasiv und für klinische Zwecke gut geeignet. Sie hat sich etabliert, da sie relativ genau, reproduzierbar, schnell, angenehm und preisgünstig ist (Wirth, 2000; Bosy-Westphal et al., 2006). Sie wurde aufgrund der Studienanforderungen sowie der Erfahrungen des Untersuchers angewendet.

Den Messungen durch das in der Studie verwandte Gerät liegt das 4Kompartimentenmodell zugrunde. Die folgende Abbildung stellt die Kompartimente wie auch die angenommen Mittelwerte dar: 


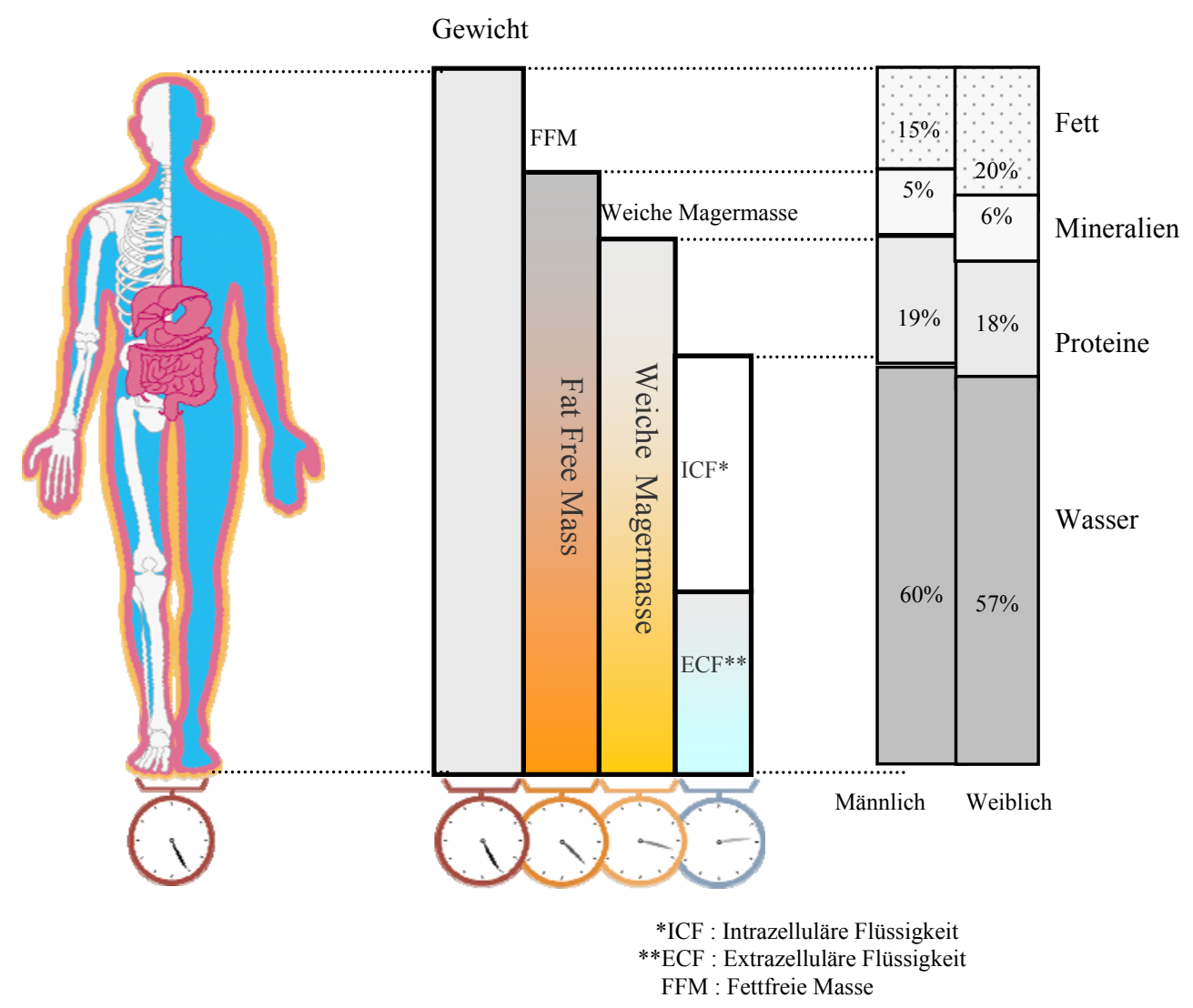

Abbildung 1: Körperzusammensetzung nach dem 4-Kompartimentmodell (Biospace 2001)

Schwäche der BIA- Methode ist die vereinfachte Annahme, der Körper sei ein Zylinder und werde gleichmäßig von angelegtem Strom durchdrungen. Der Strom fließt nur zu ca. 10 \% durch den Rumpf, d.h. es werden nur einzelne Körperareale erfasst, deren Ergebnisse auf den gesamten Körper bzw. auf bestimmte Körperkompartimente (z.B. Körperfettmasse) extrapoliert werden (Wirth, 2000; Wagner, 2005).

Ein weiteres Problem bei der Verwendung der Methode ist die Wahl einer Regressionsgleichung zur Berechnung der Körperzusammensetzung aus den Widerstandswerten. Es muss aus einer Vielzahl von in der Literatur angebotenen Gleichungen eine passende für die Studienpopulation gewählt werden. Die Ergebnisse variieren in Abhängigkeit der gewählten Regressionsgleichung. Nach Wirth (2000) sollen Formeln eingesetzt werden, die zum einen an Adipösen evaluiert wurden und zum anderen geschlechtspezifisch sind.

Die Definition der Adipositas soll aber nicht auf die medizinische Sichtweise mit alleiniger Betrachtung des Körpergewichts und der Körperzusammensetzung und 
deren Messung beschränkt bleiben. Adipositas ist ein komplexer Zustand mit psychosozialen Dimensionen, eine chronische Krankheit mit eingeschränkter Lebensqualität und hohem Morbiditäts- und Mortalitätsrisiko, die eine langfristige Betreuung erfordert (WHO, 2000).

\subsection{Adipositas als Krankheit}

Obwohl Adipositas von der WHO als eine „chronische Krankheit“ bezeichnet wird, wird sie in der Medizin über die weltweit üblichen Diagnoseschlüssel (DSM-IV und ICD-10) nicht als eine eindeutige Krankheit definiert (Kossmann, Aidelsburger, \& Wasem, 2006).

Da ihrer Entstehung grundsätzlich keine psychopathologischen Faktoren zugeordnet werden können, gilt sie im Gegensatz zur Anorexia nervosa und Bulimia nervosa nicht als Essstörung (ICD-10, Kapitel V). Sie wird in Kapitel IV als „Adipositas und sonstige Überernährung“ unter E65 bis E68 genannt (WHO, ICD-10, 2006).

„Die überwiegende Anzahl von Leitlinien bezeichnet Adipositas als Krankheit, andere beschreiben Adipositas zumindest als Risikofaktor für schwerwiegende Erkrankungen und zeigen darüber hinaus oft die gesellschaftliche Bedeutung der ansteigenden Prävalenz der Adipositas auf. Wenn die Aussage begründet wird, dass es sich bei Adipositas um eine Krankheit handelt, fallen vorwiegend Argumente, dass bei Vorliegen einer Adipositas das Risiko schwerwiegende Erkrankungen zu erleiden steigt und das Mortalitätsrisiko stark erhöht ist“ (Kossmann et al., 2006, S. 10).

Nach Hebebrand et al., (2004) fasst die Medizin Adipositas primär als einen Risikofaktor auf und weniger als eigenständige Krankheit.

Aktuell ergibt sich in der Literatur kein klares Bild zum Status der Adipositas.

\subsection{Epidemiologie und Prävalenz der Adipositas}

Übergewicht und Adipositas sind in der Bevölkerung weit verbreitet. Etwa jeder dritte erwachsene Bundesbürger ist deutlich übergewichtig und sollte aus medizinischen Gründen an Gewicht abnehmen (DAG, 2006).

"Das Durchschnittsgewicht der Deutschen hat in den letzten Jahren weiter zugenommen; in Deutschland ist jeder 2. Erwachsene übergewichtig und jeder 5. adipös“ (Wirth 2000, S. 56).

Diese Fakten verdeutlichen die Dimensionen der Adipositas auf nationaler Ebene. 
Nach der WHO sind global viele Teile der Welt von Adipositas ("globesity") betroffen. Wird nicht sofort etwas unternommen, werden Millionen unter einer Reihe von ernsthaften Erkrankungen leiden.

Im Jahr 1995 wurde die Zahl der adipösen Erwachsenen weltweit auf 200 Millionen geschätzt und 18 Millionen Kinder unter 5 Jahren waren als übergewichtig klassifiziert. Im Jahr 2000 übertraf die Anzahl der erwachsenen Menschen mit Adipositas die 300 Millionen.

Im Gegensatz zu der bislang verbreiteten Ansicht beschränkt sich die Adipositasepidemie nicht nur auf die industrialisierten Gesellschaften. Es wird geschätzt, dass über 115 Millionen Menschen in Entwicklungsländern unter mit Adipositas verbundenen Gesundheitsproblemen leiden. Während das Problem jahrzehntelang mehr oder weniger auf die wohlhabenden Industrieländer beschränkt gewesen sei, beobachte man in jüngster Zeit einen Anstieg der ernährungsbedingten Krankheiten in Ländern wie Indien und China. Dort seien vor allem die Mittel- und Oberschicht betroffen. Die Ursachen seien ähnlich wie in den Industrienationen: zu wenig körperliche Bewegung und ein Überangebot an Lebensmitteln (WHO, 2003; Munsch, 2002). 2003 litten weltweit rund eine Milliarde Patienten an starkem Übergewicht, teilte die WHO in Genf mit.

Obwohl Männer im Allgemeinen höhere Raten an Übergewicht haben, haben Frauen häufiger Adipositas (WHO, 2003; Munsch, 2002).

Die Angaben zur Prävalenz des Übergewichts und der Adipositas schwanken je nach Land, Alter, Geschlecht und Untersuchungsmethoden stark. Befragungen ergeben stets niedrigere Werte als Messungen, weil die Befragten ihr Körpergewicht oft unterschätzten und ihre Körpergröße überschätzten. Beides führt zu einer Unterschätzung des BMI, der zur Bestimmung des Übergewichts und der Adipositas herangezogen wird (Ehrsam, Stoffel, Mensink \& Melges, 2004).

Betrachtet man verschiedene epidemiologische Untersuchungen in Deutschland in den letzten 20 Jahren (vgl. Tabelle 3), stellt man fest, dass die Prävalenz der Adipositas (BMI $\geq 30)$ kontinuierlich zunimmt. 
Tabelle 3: Prävalenz von Übergewicht und Adipositas in Deutschland (nach Ehrsam, 2004, 280); Normalschrift: Studien, bei denen Messdaten erhoben wurden

Kursiv gedruckt: Studien, die auf Befragungen beruhen

\begin{tabular}{|l|c|c|c|c|c|}
\hline Deutschland & Jahr & \multicolumn{4}{|c|}{ Prävalenz } \\
\hline & & \multicolumn{2}{|c|}{ BMI 25-29,9 } & \multicolumn{2}{|c|}{ BMI $\geq 30$} \\
\hline & & Männer & Frauen & Männer & Frauen \\
\hline $\begin{array}{l}\text { PROCAM } \\
\text { PROspective CArdiovascular } \\
\text { Münster Study (1,2) }\end{array}$ & 1985 & 44,0 & 24,6 & 8,6 & 9,3 \\
\hline $\begin{array}{l}\text { DHP:NUS } \\
\text { Deutsche Herz-Kreislauf- } \\
\text { Präventions-Studie: Nationaler } \\
\text { Untersuchungssurvey }\end{array}$ & 1986 & 50,5 & 32,7 & 15,5 & 17,0 \\
\hline $\begin{array}{l}\text { DHP:NUS } \\
\text { Deutsche Herz-Kreislauf- } \\
\text { Präventions-Studie: Nationaler } \\
\text { Untersuchungssurvey }\end{array}$ & 1991 & 49,7 & 32,4 & 17,5 & 19,6 \\
\hline $\begin{array}{l}\text { NUSow } \\
\text { Nationaler Untersuchungssurvey } \\
\text { Ost West }\end{array}$ & 1992 & 49,4 & 32,2 & 18,1 & 20,9 \\
\hline $\begin{array}{l}\text { BGS } \\
\text { Bundes - Gesundheitssurvey }\end{array}$ & 1998 & 48,8 & 31,3 & 19,1 & 21,4 \\
\hline $\begin{array}{l}\text { Mikrozensus } \\
\text { Fragen zur Gesundheit }\end{array}$ & 1999 & 44,8 & 28,3 & 12,6 & 11,4 \\
\hline $\begin{array}{l}\text { Mikrozensus } \\
\text { Fragen zur Gesundheit }\end{array}$ & 2003 & 44,4 & 28,3 & 14,1 & 12,5 \\
\hline
\end{tabular}

Nach der aktuellsten für Deutschland repräsentativen Datenerhebung im Rahmen des Telefonischen Gesundheitssurvey von 2003 sind etwa $50 \%$ der erwachsenen Männer mit einem BMI $\geq 25$ übergewichtig und ca. $17 \%$ mit einem BMI $\geq 30$ adipös. Bei den erwachsenen Frauen sind etwa $31 \%$ übergewichtig und knapp $20 \%$ adipös. Derzeit sind etwa $70 \%$ der Männer und $50 \%$ der Frauen übergewichtig oder adipös (Mensink, Lampert \& Bergmann, 2005).

Anhand der vorliegenden statistischen Angaben kann zusammenfassend festgestellt werden, dass die Prävalenz der Übergewichtigen in Deutschland bei den Männern und den Frauen über die Jahre hinweg etwa gleich geblieben ist. Der Anteil der Adipösen mit einem BMI $\geq 30$ hat jedoch deutlich zugenommen. Nach den o.g. Quellen hat sich die Prävalenz adipöser Männer und Frauen innerhalb von 20 Jahren verdoppelt.

Die Weltgesundheitsorganisation (WHO) warnt davor, dass die Zahl der adipösen Patienten weltweit in der nächsten Dekade um bis zu 50 Prozent ansteigen dürfte. 
Die WHO rechnet vor, dass, sollte sich der derzeit beobachtete Trend fortsetzen, im Jahr 2015 bereits mehr als 2,3 Milliarden Erwachsene weltweit stark übergewichtig und 700 Millionen adipös sein werden. Neben den damit verbreiteten gesundheitlichen Problemen ergeben sich somit immense finanzielle Belastungen, wenn es nicht gelingt diesem Trend entgegenzuwirken (WHO, 2006).

In Deutschland besteht ein enger Zusammenhang zwischen dem Gewicht und dem sozialen Status, wie in anderen Industriestaaten auch. Je niedriger die soziale Schicht und der Bildungsgrad, desto höher ist das Gewicht. Hiervon sind vor allem Frauen betroffen: Frauen mit Hauptschulabschluss weisen vier Mal häufiger eine Adipositas auf als Frauen mit Abitur oder vergleichbarem Schulabschluss (Mensink, et al. 2005; Bergmann, Bergmann, \& Richter 2005; Valdez \& Williamson, 2002).

Um die gesellschaftliche und gesundheitspolitische Bedeutung der Adipositas besser einschätzen zu können, bedarf es neben der Kenntnis um die epidemiologischen Daten der Berücksichtigung der entstehenden Kosten durch die Erkrankung.

\subsection{Sozioökonomische Faktoren der Adipositas}

Nach der WHO führt Adipositas zu einer beträchtlichen ökonomischen Belastung. durch Produktivitätsausfälle und Einkommensverluste und ist für 2-8 \% der gesamten Gesundheitsausgaben verantwortlich (WHO, 2005).

Nach eine US Studie liegen die medizinischen Behandlungskosten bei Menschen mit Adipositas um 50 \% über denen von Normalgewichtigen (Hebebrand, 2004).

Hauptkostenfaktor im Gesundheitswesen stellen dabei die große Anzahl der Präadipösen, Adipösen und deren erhöhte Komorbidität (siehe S. 38, Tabelle 5) sowie die beeinträchtigte Lebensqualität und die damit verbundene vorzeitige Berentung dar.

D.h. Kosten entstehen zum einem durch die Inanspruchnahme medizinischer Leistungen und zum anderen durch krankheitsbedingte Arbeitsausfälle. Hinzu kommen die Ausgaben, die die Patienten selbst tätigen, z.B. für Gewichtsabnahmeprogramme, Diätprodukte oder Schlankheitsmittel.

Die Folgekosten lassen sich wie folgt klassifizieren:

- Direkte Kosten (Ressourcenverbrauch): Sie stehen durch diagnostische, therapeutische, präventive und rehabilitative Maßnahmen in unmittelbarem Zusammenhang mit der Krankheit. 
- Indirekte Kosten (Ressourcenverlust): Arbeitsunfähigkeit, vorzeitige Berentung und vorzeitiger Tod mindern die Ressourcen einer Gesellschaft. $\mathrm{Zu}$ erfassen sind hierbei auch geringere berufliche Aufstiegschancen oder der Zeitaufwand der Angehörigen für die Versorgung des erkrankten Angehörigen.

- Intangible Kosten: Psychische und soziale Auswirkungen einer Krankheit, verminderte Lebensqualität, Befindlichkeitsstörungen und Schmerzen verursachen Kosten beim Betroffenen und dessen Angehörigen (Lauterbach, Hauner, Westenhöfer \& Wirth, 1998; Wolf, 2002).

In den bisherigen durchgeführten internationalen Studien zu Krankheitskosten wurden die direkten Behandlungskosten vollständig und die indirekten Kosten nur unvollständig berechnet. Die intangiblen Kosten wurden nicht berücksichtigt. Dies führt zu einer systematischen Unterschätzung der adipositasbedingten Kosten.

Wirth (2000) bemängelt, dass es über die Höhe der Gesundheitskosten in Deutschland keine verlässlichen Angaben gibt. Die Kosten werden von Gesundheitsökonomen nach unterschiedlichen Modellen berechnet. Außerdem berücksichtigen veröffentlichte Kosten über ernährungsbedingte Krankheiten den Aspekt Adipositas ungenügend, so dass hierzu keine fundierten Aussagen gemacht werden können. Es werden die Kosten der Folgekrankheiten aufgeführt, nicht jedoch die Adipositas selbst. Selbst eine Auswertung von Krankenakten würde wenig aufschlussreich sein, da die Diagnose Adipositas nur ganz selten explizit genannt wird (Wirth 2000).

So gibt es bislang in Deutschland nur eine Veröffentlichung zu den Krankheitskosten der Adipositas (Schneider, 1996, zitiert nach Lauterbach et al., 1998). Auch diese wird als nur eingeschränkt aussagekräftig beurteilt (Lauterbach et al., 1998). In Schneiders Studie werden die Kosten aufgrund mangelhafter Datenlagen bzgl. der Prävalenzen, der Begleit- und Folgeerkrankungen und den attributablen Risiken auf der Basis zweier Modellvarianten geschätzt. Schätzungen der direkten und indirekten Krankheitskosten der Adipositas und der Folge- bzw. Begleiterkrankungen für 1995 ergeben je nach Modellvariante zwischen 7,75 und 13,55 Milliarden Euro, das sind 3,1 \% bis 5,5\% der Gesamtkosten (Benecke \& Vogel, 2003).

Wirth (2003) überträgt aufgrund vergleichbarer Prävalenzen Schätzungen und Berechnungen amerikanischer Studien auf Deutschland und kommt auf 
adipositasbedingte Gesundheitskosten von ca. 18 Mrd. Euro. Das macht ca. $6 \%$ der Gesamtkosten im Gesundheitssystem aus (Wirth, 2003).

Die Ergebnisse dieser Schätzungen stimmen relativ gut mit internationalen Krankheitskostenstudien zur Adipositas überein. Nach diesen Studien wurde der Anteil der adipositasbezogenen Kosten an den gesamten Gesundheitsausgaben auf zwischen 2-8 \% berechnet (Lauterbach et al., 1998).

Eine weitere amerikanische Veröffentlichung von Thompson und Wolf (2001) zeigt geschätzte direkt adipositasbezogene Kosten aus dem Jahr 1995 in Höhe von 70 Milliarden Dollar im Vergleich zu den Kosten anderer Krankheiten, wie koronarer Herzerkrankungen (50,8 Mrd.), Bluthochdruck, (15,6 Mrd.), Schlaganfall (18,1 Mrd.) und Typ-2-Diabetes-mellitus (53,2 Mrd.). Es wird deutlich, dass die durch Adipositas verursachten Kosten deutlich höher liegen. Auch für Deutschland ist es nach Hebebrand (2004) offensichtlich, dass adipositasassoziierte Kosten das Gesundheitssystem zu sprengen drohen.

Die medizinischen und volkswirtschaftlichen Kosten sind immens und es ist unverständlich, dass die Adipositas als Krankheit nicht voll anerkannt und ungenügend oder oft sogar gar nicht behandelt wird.

\section{5 Ätiologie}

Die ätiologischen Modellvorstellungen zur Entstehung einer Adipositas haben sich in den letzen Jahren geändert. Genetisch orientierte Ansätze werden aktuell genau so diskutiert wie die früheren dominierenden psychogenen Annahmen. Die dichotome Denkweise zur Genese der Adipositas im Sinne von „nature versus nurture“ ist zunehmend einem multifaktoriellen Genesemodell gewichen (Herpertz \& Senf, 2003). Der Phänotyp Adipositas wird heute als Ergebnis der Interaktion von genetischer bzw. evolutionärer Prädisposition und Umweltfaktoren verstanden.

Zunächst ist Adipositas die Konsequenz einer anhaltenden positiven Energiebilanz.

Die einfache mathematische Formel zur Beschreibung einer ausgeglichenen Energiebilanz lautet:

$$
\text { Energieaufnahme }=\text { Energieabgabe }
$$

In der Adipositas besteht hier ein Ungleichgewicht. Es spielen sowohl bei der Energieaufnahme als auch in der Energieabgabe multiple Faktoren eine Rolle. Diese Faktoren werden durch verhaltensbezogene und biologische Einflüsse gesteuert 
(Herpertz \& Senf, 2003). So werden in dem aktuellen Erklärungsansatz nach Lehrke und Laessle (2003) genetische Ursachen, die positive Energiebilanz, das Essverhalten sowie die körperliche Aktivität zu einem biopsychosozialen Modell der Adipositasgenese zusammengefasst (siehe Abbildung 2).

Bei der Entstehung der Adipositas ergibt sich ein Zusammenspiel von nicht veränderbarer individueller genetischer Prädisposition und im Gegensatz dazu beeinflussbaren Faktoren.

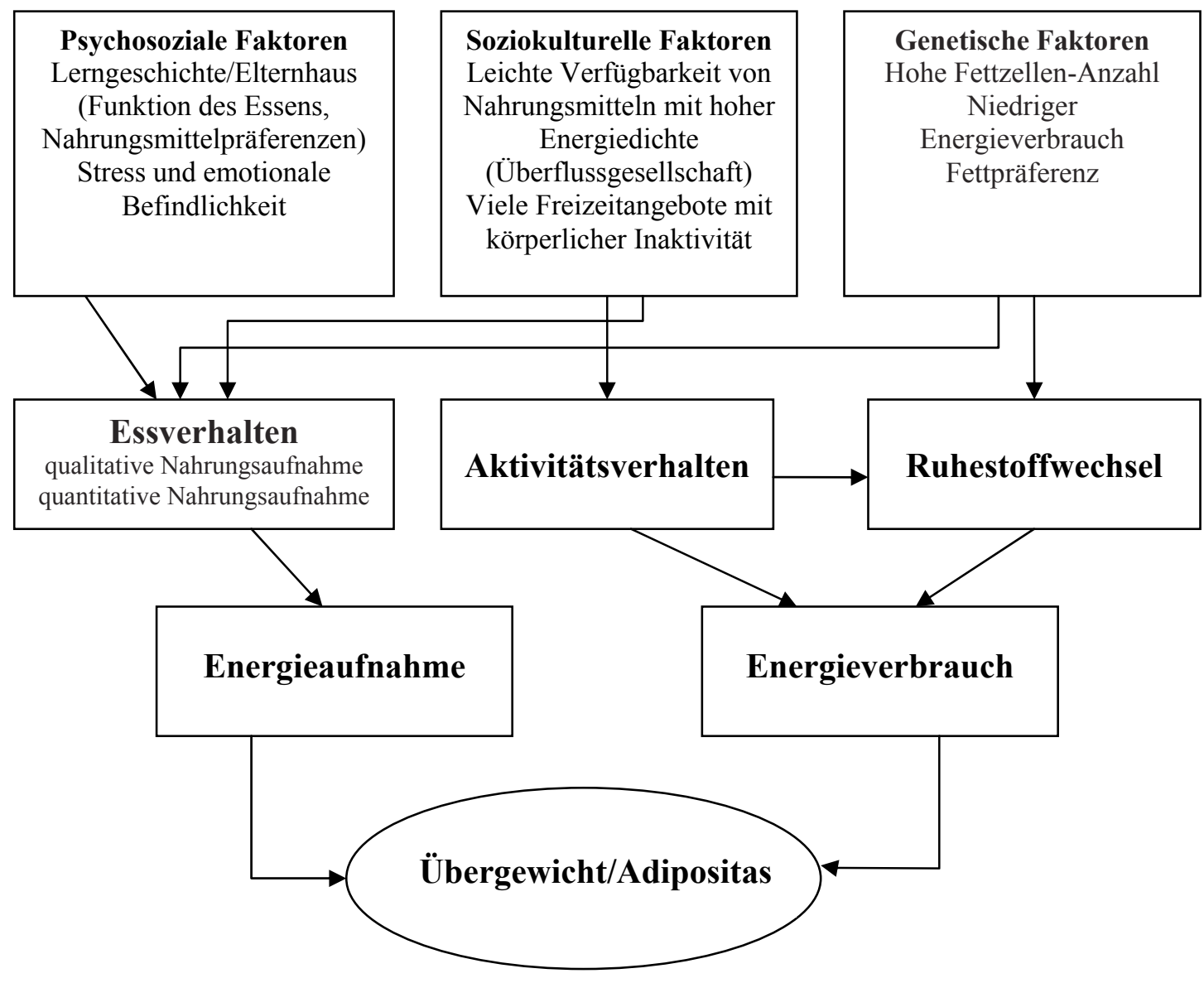

Abbildung 2: Biopsychosoziales Modell der Entstehung und Aufrechterhaltung von Übergewicht/Adipositas (Lehrke \& Laessle, 2003, 511)

Nach einem weiteren Erklärungsansatz von Salbe und Ravussin (2000) werden die Ursachen einer Adipositas in verhaltensbezogene, metabolische und biologische Kategorien eingeteilt. 


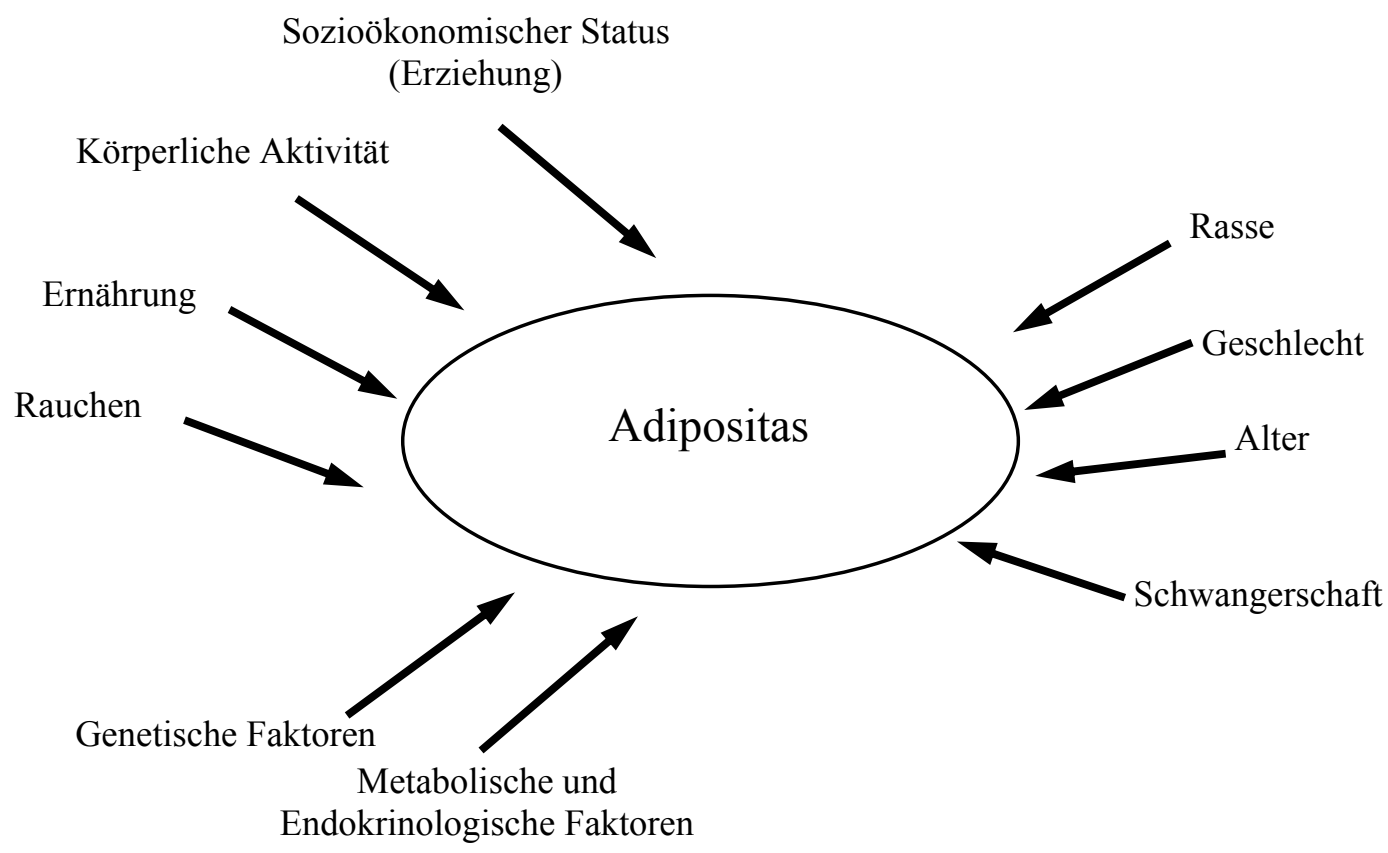

Abbildung 3: Verschiedene Adipositaskausalitäten gruppiert nach Verhalten, Stoffwechsel und biologischen Einflussgrößen (Salbe \& Ravussin, 2000, 71)

Bouchard (2000) listet eine Reihe von Einflussgrößen auf, die eine enge Beziehung zur Adipositas haben und kommentiert diese. Einige sind Prädiktoren für eine Gewichtszunahme und werden als Risikofaktoren definiert. Bei anderen Faktoren ist die Unterscheidung einer primären oder nur sekundären Kausalität nicht möglich. Viele dieser Korrelate können als Folge eines Adipositasstatus gesehen werden. 
Tabelle 4: Korrelate von Übergewicht und Adipositas oder Körpergewicht und Körperfettzunahme über die Zeit (Bouchard, 2000, 8)

\begin{tabular}{|c|c|}
\hline Variable & Erläuterung \\
\hline Alter & $\begin{array}{l}\text { - Adipositas im Kindesalter ist ein Risikofaktor für } \\
\text { Adipositas im Erwachsenenalter. } \\
\text { - Der Körperfettgehalt steigt während des } \\
\text { Erwachsenenalters. } \\
\text { - Maximale Höhe des Übergewichts und Adipositas } \\
\text { werden im Alter von } 55 \text { - 65 Jahren erreicht. }\end{array}$ \\
\hline Geschlecht & $\begin{array}{l}\text { - Frauen haben mehr Körperfett. } \\
\text { - Die Geschlechterunterschiede in der Prävalenz } \\
\text { von Adipositas variieren sowohl in der } \\
\text { Bevölkerung als auch zwischen den ethnischen } \\
\text { Gruppen. }\end{array}$ \\
\hline Soziökonomischer Status & $\begin{array}{l}\text { - Mehr Adipöse in Schichten mit hohem } \\
\text { sozioökonomischem Status in armen Ländern. } \\
\text { - Mehr Adipöse in Schichten mit niedrigem } \\
\text { sozioökonomischem Status in reichen Ländern. }\end{array}$ \\
\hline Energieaufnahme & $\begin{array}{l}\text { - Überernährung verursacht Gewichtzunahme und } \\
\text { führt zur Adipositas. }\end{array}$ \\
\hline $\begin{array}{l}\text { Aufnahme von } \\
\text { Nahrungsfett }\end{array}$ & $\begin{array}{l}\text { - Nahrungsfett steht mit der Prävalenz von } \\
\text { Übergewicht in engem Zusammenhang. } \\
\text { - Diäten mit hohem Fettgehalt verursachen } \\
\text { Gewichtszunahme. } \\
\text { - Diäten mit niedrigem Fettgehalt reduzieren das } \\
\text { Körpergewicht. }\end{array}$ \\
\hline Grundumsatz & $\begin{array}{l}\text { - Ein im Verhältnis zu Körpermasse und } \\
\text { Körperzusammensetzung niedriger Grundumsatz } \\
\text { ist ein Prädiktor für eine Gewichtszunahme, hier } \\
\text { gibt es jedoch widersprüchliche Ergebnisse. } \\
\text { - Übergewichtige und Adipöse haben einen höheren } \\
\text { absoluten Grundumsatz. }\end{array}$ \\
\hline Thermogenese & $\begin{array}{l}\text { - Es ist immer noch umstritten, ob und in welchem } \\
\text { Umfang bei Adipösen eine verminderte } \\
\text { nahrungsinduzierte Wärmebildung vorliegt. }\end{array}$ \\
\hline $\begin{array}{l}\text { Ausmaß körperlicher } \\
\text { Aktivität }\end{array}$ & $\begin{array}{l}\text { - Ein niedriger Level an körperlicher Aktivität ist } \\
\text { ein Risikofaktor für eine Gewichtszunahme. } \\
\text { - Das Ausmaß an Inaktivität ist bei den Adipösen } \\
\text { höher. } \\
\text { - Regelmäßige körperliche Aktivität verändert die } \\
\text { Körperzusammensetzung. } \\
\text { - Hohe Level körperlicher Aktivität steigern die } \\
\text { Sympathikusaktivität und den Grundumsatz. } \\
\text { - Regelmäßige körperliche Aktivität trägt zur } \\
\text { Gewichtsabnahme und Gewichtsaufrecherhaltung } \\
\text { bei. }\end{array}$ \\
\hline
\end{tabular}




\begin{tabular}{|c|c|}
\hline Fettoxidationsrate & $\begin{array}{l}\text { - Eine Körperfettzunahme mindert den } \\
\text { Respiratorischen Quotienten. } \\
\text { - Ein hoher Respiratorischer Quotient ist ein } \\
\text { Risikofaktor für eine Gewichtszunahme, hier gibt } \\
\text { es jedoch widersprüchliche Ergebnisse. } \\
\text { - Ehemalige Adipöse haben einen höheren } \\
\text { Respiratorischen Quotienten als Menschen, die nie } \\
\text { adipös waren. }\end{array}$ \\
\hline Leptinspiegel im Blut & $\begin{array}{l}\text { - Niedrige Leptinspiegel werden schwach mit einer } \\
\text { Gewichtszunahme verbunden. Hier gibt es jedoch } \\
\text { sich widersprechende Ergebnisse. } \\
\text { - Die meisten Adipösen haben hohe Leptinspiegel. }\end{array}$ \\
\hline Sympatikusakt & $\begin{array}{l}\text { - Niedrige Aktivität des sympathischen } \\
\text { Nervensystems könnte ein Risikofaktor für eine } \\
\text { Gewichtszunahme sein. } \\
\text { - Die Aktivität des sympathischen Nervensystems } \\
\text { steigt mit Überernährung und Gewichtszunahme. }\end{array}$ \\
\hline Wachstu & $\begin{array}{l}\text { - Niedrige Wachstumshormonspiegel sind ein } \\
\text { Risikofaktor für eine Gewichtszunahme. } \\
\text { - Die meisten Adipösen haben niedrige } \\
\text { Wachstumshormonspiegel. }\end{array}$ \\
\hline Insul & $\begin{array}{l}\text { - Bei Adipösen besteht oft Insulinresistenz und } \\
\text { Hyperinsulämie. } \\
\text { - Die Insulinresistenz schützt vor einer } \\
\text { Gewichtszunahme, hier gibt es jedoch } \\
\text { widersprüchliche Ergebnisse. }\end{array}$ \\
\hline $\begin{array}{l}\text { Hypothalamus - } \\
\text { Hypophysen- } \\
\text { Nebennieren-Achse } \\
\text { (HPA-Achse) und } \\
\text { Cortisolspiegel }\end{array}$ & $\begin{array}{l}\text { - Adipöse haben im Allgemeinen eine } \\
\text { überreagierende und hyperaktive HPA-Achse. } \\
\text { Adipöse haben eine erhöhte Cortisolausschüttung, } \\
\text { aber auch einen beschleunigten Cortisolabbau. }\end{array}$ \\
\hline Gesc & $\begin{array}{l}\text { - Adipöse Männer haben häufig niedrige } \\
\text { Androgenspiegel. } \\
\text { - Adipöse Frauen haben häufig hohe } \\
\text { Androgenspiegel und eine erhöhte Stimulation des } \\
\text { Adrenocortikotropen Hormons (ACTH) }\end{array}$ \\
\hline $\begin{array}{l}\text { Meta } \\
\text { Fettg }\end{array}$ & 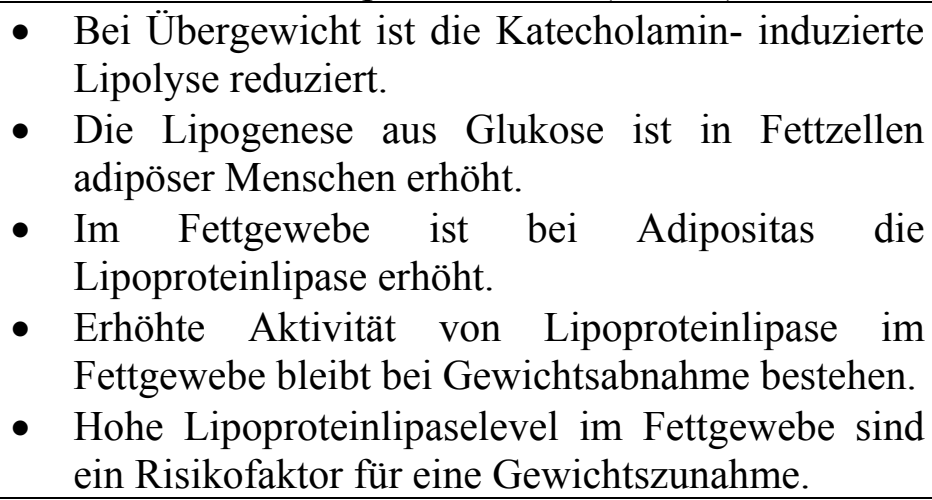 \\
\hline $\begin{array}{l}\text { Metabolismus der } \\
\text { Skelettmuskulatur }\end{array}$ & $\begin{array}{l}\text { - Der Anteil von Muskelfasern vom Typ I wird } \\
\text { nicht von derAdipositas beeinträchtigt. } \\
\text { - } \quad \text { Der Anteil von Muskelfasern vom Typ II b ist }\end{array}$ \\
\hline
\end{tabular}




\begin{tabular}{|ll|}
\hline & \multicolumn{1}{|l|}{ häufig bei Adipositas erhöht. } \\
& Oxidative Enzymmarker in der Skelettmuskulatur \\
& stehen im umgekehrten Verhältnis zur Adipositas. \\
& Die Aktivität der Lipoproteinlipasen in der \\
& Skelettmuskulatur ist bei Adipositas niedrig. \\
\hline $\begin{array}{l}\text { Energie und Nährstoffe } \\
\text { Aufteilung }\end{array}$ & Bei positiver Energiebilanz wandeln manche \\
& Personen mehr Kohlenstoff aus der Nahrung in \\
& Proteine um. \\
& Eine hohe Rate des Lipidzuwachs kann ein \\
& Risikofaktor für eine weitere Gewichtszunahme \\
& sein. \\
\hline Rauchen & Rauchen ist mit niedrigem Körpergewicht \\
& assoziiert. \\
& Beenden des Rauchens erhöht bei den meisten \\
& Menschen das Körpergewicht. \\
\hline
\end{tabular}

Das Ausmaß der körperlichen Bewegung ist der im Zusammenhang mit der vorliegenden Arbeit bedeutendste Aspekt und wird aus diesem Grund im Folgenden ausführlicher dargestellt.

\subsubsection{Körperliche Aktivität als Entstehungsursache der Adipositas}

Einer der wichtigsten ätiologischen Faktoren, welcher in jedem Erklärungsansatz erwähnt wird, ist die niedrige körperliche Aktivität. Geringe körperliche Aktivität als Adipositasursache ist eine einleuchtende wie facettenreiche Hypothese. Sie gehört zu den so genannten Umweltfaktoren, deren Steuerung verhaltensbezogen ist. Geringe Aktivitätslevel werden in erster Linie als Ursache per se genannt, können jedoch auch als Folge der Adipositas gesehen werden (Platte, 2003). Die Energieausgabe durch körperliche Aktivität ist variabel. Ansteigende Adipositas verläuft parallel zu einer inaktiven, sitzenden Lebensweise.

Als Evidenz für diese These können die Befunde einer Studie von Prentice und Jepp (1995) aus Großbritannien herangezogen werden. Ihrer Studie zufolge nahm in den letzten drei Dekaden die Adipositasrate trotz einer nachweisbaren Abnahme der Energiezufuhr dramatisch zu. Die Forscher erklären dieses Paradoxon dadurch, dass in der gleichen Zeitspanne die Minderung an körperlichen Aktivitäten noch größer war als die Minderung der Energiezufuhr. Sie belegen dieses mit Daten, die für die Aktivität bzw. Inaktivität sprechen, wie Anzahl der Stunden des Fernsehens und die Anzahl der Autos pro Haushalt. Beide Größen sind in dieser Zeitspanne angestiegen. 
Atkinson (1999) kommt zu einer ähnlichen Schlussfolgerung. Das Ausmaß an körperlicher Aktivität trägt deutlich zu der Aufrechthaltung des Körpergewichts bei. Adipöse sind weniger aktiv als magere Menschen. Der Faktor, der am höchsten mit dem Adipositasgrad korreliert, ist Inaktivität. Der Grad der Adipositas korreliert negativ mit der täglichen körperlichen Aktivität.

In einer Studie von Schulz und Schoeller (1994) mit über 200 Probanden wurde eine weite Variabilität des Gesamtenergieverbrauchs und so der körperlichen Aktivität gefunden. Auch diese Autoren konnten zeigen, dass die körperliche Aktivität bei übergewichtigen oder adipösen Probanden herabgesetzt war.

Unter körperlicher Aktivität wird nicht nur die formale freiwillige sportliche Aktivität verstanden, sondern alle körperlichen spontanen Alltagsaktivitäten (Gehen, Treppesteigen etc.). Auch „unproduktive“ muskuläre Aktivitäten wie Herumzappeln, Schaudern und Zittern werden mitberücksichtigt. Spontane alltägliche körperliche Aktivitäten spielen genau wie sportliche Aktivitäten eine wichtige Rolle beim Energieverbrauch und können für erhebliche Gewichtsunterschiede verantwortlich sein. Erhöhte spontane körperliche Aktivität nimmt eine präventive Rolle bei der Entwicklung von Adipositas ein (Levine, Eberhardt \& Jensen, 1999).

\subsubsection{Energieverbrauch und die Entstehung von Übergewicht}

Der Energieverbrauch durch die körperliche Aktivität ist die variabelste der drei Komponenten des Gesamtenergieverbrauchs:

- passive Thermogenese

- aktivitätsinduzierte Thermogenese (AIT)

- spontane körperliche Aktivitäten.

Die aktivitätsinduzierte Thermogenenese (AIT) liegt zwischen $15 \%$ und $50 \%$ der gesamten Energieabgabe und hängt von dem Ausmaß (Art, Dauer und Intensität) der ausgeführten körperlichen Aktivität und der Körpermasse des Individuums ab. Der AIT-Prozentsatz macht bei passiven Erwachsenen nur 20-30 \% der Gesamtenergieausgabe aus. (Tataranni \& Ravussin, 2004). Spontane körperliche Aktivitäten bestimmen wiederum $8-15 \%$ des totalen täglichen Energieverbrauchs (Tataranni \& Ravussin, 2004). 
Verschiedene Autoren machen unterschiedliche Angabe bezüglich des Ausmaßes des Energieverbrauchs (AIT). Die Messung der AIT ist komplizierter als die Messung des Grundumsatzes (GU) und der diätinduzierten Thermogenese (DIT).

Es wird bemängelt, dass es keine befriedigende Methode gibt, die den Einfluss der körperlichen Aktivität auf den täglichen Gesamtenergieverbrauch genau misst. Die zur Zeit beste Methode zur Messung des Gesamtenergieverbrauchs bei freien Lebensbedingungen ist die Doppel-Isotopen-Methode (doubly labeled water method). Die Methode ist sehr teuer und aus diesem Grund in großen Studien nicht einsetzbar. Es besteht allerdings Hoffnung, dass es durch diese Methode in der näheren Zukunft möglich wird, die Interaktion zwischen Energieverbrauch bei alltäglichen körperlichen Aktivitäten und der Entwicklung von Adipositas im Kindes- und Erwachsenenalter besser zu verstehen (Tataranni \& Ravussin, 2004).

Black, Coward, Cole und Prentice (1996) erstellten ein Review von 547 Messungen, die mit der Doppel-Isotopen-Methode erhoben wurden. Sie analysierten die Datenlage:

- um die Grenze der dauerhaften menschlichen Energieausgabe festzulegen,

- um Mittelwerte und Umfänge der habituellen Energieausgabe im Verhältnis zum Alter und Geschlecht festzulegen

- und um Lebensstile und aktive Verhaltensmuster zu beschreiben, die mit verschiedenen Niveaus körperlicher Aktivitäten verbunden sind.

Sie stellten fest, dass der habituelle Gesamtenergieverbrauch bei Adipositas steigt, da sich mit dem Übergewicht gleichzeitig die freie Körperfettmasse erhöht.

Die extensive Adipositas ausgenommen sei der Energieverbrauch dem Aufwand der körperlichen Aktivität in den verschiedenen Gewichtsklassen und BMI-Kategorien entsprechend gleich (Black, Coward, Cole \& Prentice, 1996).

Diese These wird von Melby, Ho und Hill (2000) nicht durchgehend unterstützt. Sie betonen die Rolle der Körpergröße für den Energieverbrauch in Aktivität. Die umgesetzte Energie bei 1 Meile Walking sei für einen Menschen mit einem Körpergewicht von $100 \mathrm{~kg}$ größer als für eine Person von $60 \mathrm{~kg}$ Körpergewicht.

Eine kleine magere Person die moderat aktiv ist, könne in etwa die gleichen Kalorien am Tag wie eine große adipöse Person, die inaktiv ist, verbrauchen (Melby, Ho \& Hill, 2000). 
Da die Energieausgabe sowohl bei einer Person als auch zwischen den Menschen sehr variabel ist, bedarf ihre Rolle für die Körpergewichtsregulation und die Ätiologie der Adipositas einer näheren Untersuchung (Melby, Ho \& Hill, 2000).

Um die Meinung der meisten Wissenschaftler, dass ein niedriges körperliches Aktivitätsniveau ein Haupt- und Prädispositionsfaktor für eine Gewichtszunahme bei Individuen und großen Populationen ist, eindeutig zu belegen, bedarf es prospektiver Studien. Diese sollten die körperliche Aktivität insbesondere bei freien Lebensbedingungen berechnen (Sundquist \& Johansson, 1998).

Trotz der beschriebenen Erkenntnisse hinsichtlich der Entstehung von Übergewicht ist die Adipositasgenese nicht abschließend geklärt und weitere Forschung ist indiziert. Das vereinfachte Prinzip der positiven Energiebilanz, das die Adipositas ausschließlich aufgrund einer zu hohen Energieaufnahme erklärt, wird dem komplexen Phänomen der Adipositas nicht gerecht. Die Berücksichtigung der Energieabgabe ist ein weiterer wichtiger Faktor.

Eine genetische Prädisposition heißt nicht, dass Adipositas unvermeidbar ist, denn Umweltfaktoren können entscheidend für das Nach- außen- Treten und die Durchsetzung dieses genetischen Potentials sein. Es gibt verschiedene Faktoren, wie Ernährung, Bewegungsverhalten, endokrinologische oder metabolische Erkrankungen und Pharmaka, die zur einer Adipositas führen können, jedoch manipulierbar sind (Hill, 2002).

\subsection{Medizinische Konsequenzen von Adipositas}

Nach Atkinson (1999) ist Adipositas eine außerordentlich komplexe Gruppe von Erkrankungen, die als ein Syndrom charakterisiert werden könnten.

Adipositas bekommt ihren Krankheitswert durch eine Vielzahl von Folgeerkrankungen, die erhebliche Auswirkungen auf die Morbidität der Bevölkerung haben. Die Auswirkungen des Übergewichts auf die Morbidität und Mortalität waren vor mehr als 2000 Jahren bekannt. Hippokrates stellte fest, dass "sudden death is more common in those who are naturally fat than in the lean." (Hippokrates, zitiert nach Bray, 2000, S. 31).

Wirth, (2003) gibt eine ausführliche Auflistung der mit Adipositas assoziierten Krankheiten. Assoziiert bedeutet, dass die Krankheit überzufällig häufig bei 
Adipösen vorkommt. Aus seiner somatisch- medizinischen Sicht werden von der Adipositas viele Organsysteme tangiert.

Vorwiegend betroffen sind der Stoffwechsel, die Atmung, die tragenden Gelenke und hormonabhängige maligne Zellveränderungen. Besonders eng mit der Adipositas verknüpft sind der Diabetes mellitus, der Bluthochdruck, die Fettstoffwechselstörungen, das Gallensteinleiden und die Schlafapnoe. 
Tabelle 5: Mit der Adipositas assoziierte Krankheiten (Wirth, 2003, 105)

1. Kardiovaskuläres System

- Hypertonie

- Koronare Herzkrankheit

- Linksvertikuläre Hypertrophie

- Herzinsuffizienz

- Venöse Insuffizienz

- Apoplex

2. Metabolische und hormonelle Funktion

- Diabetes mellitus Typ 2

- Dyslipidämien

- Hyperurikämie

3. Hämostase

- Hyperfibrinogenämie

- Erhöhter Plasminogen-Aktivator-Inhibitor

4. Respiratorisches System

- Schlafapnoe

- Pickwick-Syndrom

5. Gastrointestinales System

- Choleszystolithiasis

- Fettleber

- Refluxösophagitis

6. Haut

- Intertrigo

- Hirsutismus, Striae

7. Bewegungsapparat

- Koxarthrose

- Gonathrose

- Fersensporn

- Wirbelsäulensyndrome

8. Neoplasien

- Erhöhtes Risiko für Endometrium-, Mama-, Zervix-, Ösophagus u. Nierenzellkarzinom

9. Sexualfunktion

- Reduzierte Fertilität

- Komplikationen bei Geburt und post partum

- Polyzystisches Ovar-Syndrom

10. Verschiedenes

- Erhöhtes Operations- und Narkoserisiko

- Erhöhte Verletzungsgefahr

- Erschwerte Untersuchungsbedingungen

- Verminderte Beweglichkeit und Ausdauer 
Große prospektive Studien haben gezeigt, dass ein steigender BMI mit einer zunehmenden Verkürzung der Lebenserwartung verbunden ist (National Heart, Lung, and Blood Institute [NHLBI] 1998; DAG, 2006).

Es muss berücksichtigt werden, dass die Wirkungen der Adipositas auf die Gesundheit häufig nicht isoliert gesehen werden können. Komorbiditäten und Risikofaktoren (z.B. körperliche Inaktivität, Rauchen), die sich gegenseitig beeinflussen, sind bei adipösen Personen zu finden.

Weiterhin ist es nicht einfach zu definieren, ab welchem Punkt des menschlichen Körpergewichtes das Risiko beginnt (Manson, Skerrett \& Willett, 2002). Es gilt, dass die Mortalitätsrate steigt, je höher das erreichte Gewicht ist (Pi-Sunyer, 1999). Ab einem BMI $>25$ ist eine erhöhte Mortalität zu verzeichnen. Der Mortalitätsanstieg ist bis $\mathrm{zu}$ einem $\mathrm{BMI}$ von 30 nur gering. $\mathrm{Ab}$ einem $\mathrm{BMI}>30$ ist die Mortalitätszuwachsrate beträchtlich. Vor allem Patienten mit kardiovaskulären Erkrankungen sind stark betroffen. Ihr Mortalitätsrisiko liegt im Vergleich zu Personen mit einem BMI im Normbereich um 50-100 Prozent höher (Lehrke \& Laessle, 2003).

Epidemiologische Studien zeigen, dass Adipositas mehr Einwirkung auf die Mortalität bei jüngeren als bei älteren Personen hat. Mit höherem Lebensalter schwächt sich das Mortalitätsrisiko von adipösen Personen ab (Calle, Thun, Petrelli, Rodriguez \& Heath, 1999, zitiert nach DAG, 2006). Nach Flegal, Graubard, Williamson \& Gail (2005) hat sich die Wirkung der Adipositas auf die Mortalität in den letzten 30 Jahren verringert (Flegal et al., 2005, zitiert nach DAG, 2006).

\subsubsection{Metabolisches Syndrom}

Besonders bedrohlich für die Gesundheit der Betroffenen sind die Stoffwechselveränderungen, von denen das Metabolische Syndrom, das die Vorstufe des Diabetes mellitus Typ II bildet, besonders wichtig ist. Das Metabolische Syndrom zeigt die Entwicklung eines Diabetes mellitus Typ II an und ist mit einem hohen Atheroskleroserisiko assoziiert. Nach Grundy et al., (2005) liegt ein Metabolisches Syndrom vor, wenn 3 der 5 folgenden 5 Diagnosekriterien erfüllt sind. 
Tabelle 6: Kriterien für die Diagnose des Metabolischen Syndroms nach AHA/NHLBI (Grundy et al., 2005, zitiert nach DAG, 2006, 9)

Erhöhter Taillenumfang

Erhöhte Triglyzeride (nüchtern)

Bluthochdruck

Erhöhte Nüchternblutglukose
Männer $\geq 102 \mathrm{~cm}$

Frauen $\geq 88 \mathrm{~cm}$

$\geq 150 \mathrm{mg} / \mathrm{dl}(1,7 \mathrm{mmol} / \mathrm{L})$

oder

Medikamenteneinnahme zur Behandlung erhöhter

Triglyzeride

Niedriges HDL-Cholesterin (nüchtern)

Männer $<40 \mathrm{mg} / \mathrm{dl}(1,0 \mathrm{mmol} / \mathrm{L})$

Frauen $<50 \mathrm{mg} / \mathrm{dl}(1,3 \mathrm{mmol} / \mathrm{L})$

oder

Medikamenteneinnahme zur Behandlung von niedrigem

HDL- Cholesterin

$\geq 130 \mathrm{~mm} \mathrm{Hg}$ systolischer Blutdruck

$$
\text { oder }
$$

$\geq 85 \mathrm{~mm} \mathrm{Hg}$ diastolscher Blutdruck

oder

Medikamenteneinnahme zur Behandlung bestehenden

Bluthochdrucks

$\geq 100 \mathrm{mg} / \mathrm{dl}(5,6 \mathrm{mmol} / \mathrm{L})$

oder

Medikamenteneinnahme zur Behandlung erhöhter

Blutglukose

Eine Gruppe von Experten rät davon ab, das Metabolische Syndrom als eigenständiges Syndrom zu betrachten. Das so genannte „Metabolische Syndrom“ wird von diesen Fachleuten als ein Cluster kardiovaskulärer Risikofaktoren gesehen, deren klinischer Marker über die Diagnosekriterien hinaus eine muskuläre Unterempfindlichkeit gegenüber dem körpereigenen Insulin ergibt. Durch eine erworbene Insulinresistenz infolge des Übergewichts und des Bewegungsmangels wird diese bei vielen Patienten verstärkt (Mehnert, 2000).

\subsection{Psychosoziale Konsequenzen der Adipositas}

Die Konsequenzen der Adipositas beschränken sich nicht auf den somatischen Bereich. Weitere medizinische und psychosoziale Komplikationen, wie sie beispiels- 
weise von der Deutschen Adipositas Gesellschaft (DAG) genannt werden, vervollständigen das Bild der Auswirkungen und verdeutlichen die Komplexität der Erkrankung:

- Allgemeinbeschwerden (z. B. verstärktes Schwitzen, Gelenkbeschwerden, Belastungsdyspnoe),

- Einschränkung der Aktivitäten des täglichen Lebens (ADL),

- verminderte Lebensqualität,

- erhöhtes Unfallrisiko,

- erhöhtes Komplikationsrisiko während der Schwangerschaft (z.B. Eklampsie, Gestationsdiabetes) und vor und nach der Entbindung (z.B. Sektiorate, Nachblutungen),

- psychosoziale Konsequenzen mit erhöhter Depressivität und Ängstlichkeit, soziale Diskriminierung, Selbstwertminderung, soziale Isolation

(DAG, 2006, S. 8).

Die psychosozialen Folgeerscheinungen der Adipositas sind neben den medizinischen und somatischen sehr belastend für die Betroffenen.

Nach Wirth sind psychosoziale Nachteile oft gravierender als Krankheiten (Wirth, 2003). Häufig werden Menschen mit Adipositas sowohl im beruflichen als auch im privaten Bereich diskriminiert und sozial ausgegrenzt.

Die Ursache liegt für Pudel (1998) in dem Erscheinungsbild der Adipositas. Die Fettleibigkeit lässt sich nicht verstecken wie andere Erkrankungen, sondern ist stets offen für alle zu sehen. Hinzu kommt, „dass Adipositas nicht als Krankheit, sondern als schuldhaft selbst verursachter Zustand bewertet wird“ (Pudel, 1998, S. 200).

Gesellschaftliche Vorurteile gegenüber Menschen mit Übergewicht beginnen bereits im Kindesalter. Sehr aussagekräftig ist die Studie von Staffieri (1967), der die Einstellung zu adipösen Jugendlichen in den 60er Jahren untersuchte (Staffieri, 1967, zitiert nach Wadden, Womble, Stunkard \& Anderson, 2004). Nach seiner Studie werden adipöse Kinder von präpubertären Kindern als „faul“, „hässlich“, „dumm“, „dreckig“ und „verlogen“ bezeichnet. Diese negative Attitüde setzt sich ins Erwachsenenalter fort. Dickere Menschen gelten als unattraktiv, kommen weniger für ein Rendezvous in Frage und ihr Übergewicht wird als ein bedeutsames Hindernis für die Ehe angesehen (Lehrke \& Laessle, 2003; Wadden et al., 2004). 
Die Vorurteile gegen Personen mit Adipositas weiten sich bis in medizinische Berufe hinein aus. Zahlreiche Studien berichteten, dass Mediziner negative Einstellungen gegenüber Patienten mit Adipositas haben (Puhl \& Brownell, 2002; Wadden et al., 2004).

Diskriminierung zeigt sich auch am Arbeitsplatz. Es werden weniger adipöse Frauen eingestellt, ihre Leistungsfähigkeit wird negativer bewertet, und sie werden seltener befördert (Puhl \& Brownell, 2002; Wadden et al., 2004).

In einer Studie von Falkner et al., (1999) mit einer Stichprobengröße von etwa 1000 Personen, die an einem präventiven Gewichtsreduktionsprogramm teilnahmen, berichten $22 \%$ der Frauen und $17 \%$ der Männer von sozialer Diskriminierung aufgrund ihres Gewichtes.

Auch eine Untersuchung von Gortmaker, Must, Perrin, Sobol und Dietz (1993) zeigt die soziale Benachteiligung adipöser Menschen sehr deutlich. Bei den Frauen sind die Auswirkungen der Adipositas offensichtlicher als bei den Männern. Nach den Ergebnissen dieser Studie verfügen Menschen mit Übergewicht über eine niedrigere Schulbildung, haben ein geringeres Einkommen und Schwierigkeiten eine Anstellung zu finden. Auch Gortmarkers Studie zeigt, dass Adipöse nicht die gleichen Chancen wie Normalgewichtige bezüglich des beruflichen Aufstiegs haben.

Im Hinblick auf den privaten Bereich wurde gezeigt, dass übergewichtige Frauen etwa $20 \%$ und übergewichtige Männer etwa $11 \%$ seltener heirateten als Normalgewichtige.

Ebenso frühere Untersuchungen von Wadden und Stunkard (1985) und Holme und Sorensen (1986) belegen deutlich, dass adipöse Menschen in der heutigen Gesellschaft öffentlich diskriminiert werden und Vorurteilen ausgesetzt sind (Puhl \& Brownell, 2002; Wadden et al., 2004).

Katamnestische Untersuchungen nach einer Gewichtsreduktion zeigen fast einheitlich, dass psychosoziale Probleme überwiegend Folge und nicht Ursache der Adipositas sind, da eine Gewichtsreduktion allein zu einer drastischen Verbesserung psychischer Symptome führt (Herpertz \& Senf, 2003).

\subsubsection{Adipositas und gesundheitsbezogene Lebensqualität (HRQL)}

Lebensqualität ist ein breiter Begriff, der physische, psychische und soziale Gesundheit einschließt. 
Gesundheitsbezogene Lebensqualität (health-related quality of life HRQL) ist ein engerer Begriff. Fontaine und Bartlett (2003, S. 59) geben folgende Definition: „HRQL reflects an individual's subjective evaluation and reaction to health or illness “. Die gesundheitsbezogene Lebensqualität wird durch Selbstratingfragebögen oder Interviews erfasst. Es handelt sich um subjektive Aussagen aus der Sicht der Befragten. Der Patient wird gefragt, was er tun kann (Funktionsfähigkeit) und wie er sich fühlt (Wohlbefinden), (Seidell \& Tijhuis, 2002; Fontaine \& Bartlett, 2003). Informationen über die gesundheitsbezogene Lebensqualität können die Entwicklung klinischer Prozesse, Leistungserbringungen, Ausgaben im Gesundheitswesen sowie die Politik des Gesundheitswesens beeinflussen (Kaukua, 2004).

Eine Reihe von generischen, so genannten krankheitsübergreifenden, sowie für Adipositas spezifischen Fragebögen sind entwickelt worden. Sie untersuchen verschiedene Aspekte der gesundheitsbezogenen Lebensqualität übergewichtiger Personen. Diese Fragebögen erforschen in an die Belange der Adipositas angepasster Weise die Funktionsfähigkeit und die Gesundheit in den physischen, psychischen und sozialen Bereichen (Seidell \& Tijhuis, 2002; Fontaine \& Bartlett, 2003).

Es muss jedoch betont werden, dass der Aspekt „Lebensqualität bei Adipositas“ im Gegensatz $\mathrm{zu}$ den meisten anderen chronischen Störungen bzw. Erkrankungen jahrzehntelang missachtet und wenig beforscht wurde. Erst in den letzten Jahren wurde der Faktor Lebensqualität bei Adipositas näher untersucht (Hebebrand et al., 2004).

Aktuelle Literaturübersichten (Kolotkin, Meter \& Williams, 2001; Kushner \& Foster, 2000; Kaukua, 2004) beschreiben die möglichen negativen Auswirkungen der Adipositas auf die Lebensqualität deutlich.

In Beobachtungsstudien ist Normalgewicht mit guter Lebensqualität assoziiert. Personen mit Adipositas zeigen hingegen eine verminderte Lebensqualität.

Ein hoher BMI geht mit einer niedrigen Lebensqualität einher und es wurde festgestellt, dass Adipositas größere Auswirkungen auf die physische Funktionsfähigkeit als auf die psychosozialen Aspekte bezüglich der gesundheitsbezogenen Lebensqualität hat (Fontaine \& Bartlett, 2003; Field, Barnoya \& Colditz, 2004).

Die vermehrte Körperfettmasse reduziert die körperliche Funktionsfähigkeit. Weiterhin fallen alltagsmotorische Handlungen wie Gehen, Treppensteigen, Bücken, Knien etc. schwerer oder sind gar unmöglich (Wirth, 2003). Bei Adipösen schweren 
Grades, bei Adipösen mit einem chronifizierten medizinischen Status oder in Kombination mit einer Schmerz- oder Essstörung scheint auch das psychische Befinden und die sozialen Beziehungen beeinträchtigt (Kaukua, 2004).

Verschiedene Variablen, wie Geschlecht, Alter, sozioökonomischer Status und Lebensführung, verändern das Verhältnis von Adipositas und Lebensqualität. Bei Menschen mit Adipositas, die eine Gewichtsreduktion anstreben, kommt es bei einem Ansteigen des Adipositasgrades zu umfangreichen Verschlechterungen in allen Bereichen ihrer Lebensqualität. Besonders betroffen von diesen Verschlechterungen sind Frauen. Bei den für Adipositas spezifischen Bereichen der Lebensqualität sind Männern und Frauen gleichermaßen betroffen (Kolotkin, Crosby \& Williams, 2002).

Adipöse Personen, die vor einer operativen Behandlung stehen, weisen die schlechtesten Werte bzgl. ihrer Lebensqualität auf (Seidell \& Tijhuis, 2002).

Die psychosozialen Probleme unter den morbiden Adipösen, die nach eine Therapie suchen, können ebenfalls sehr schwer sein. Häufig tritt auch eine Kombination mit einer depressiven Symptomatik auf oder es zeigen sich Zeichen einer Angststörung (Kaukua, 2004).

Es scheint, dass eine Gewichtsreduktion, erzielt durch Interventionen wie Lifestiländerungen, Diäten, Medikamente und chirurgische Behandlungsansätze, in der Lage ist, die verminderte Lebensqualität zu verbessern (Seidell \& Tijhuis, 2002; Fontaine \& Bartlett, 2003). Diese Verbesserung ist hierbei vom Umfang des Gewichtsverlustes abhängig. Je größer der Gewichtsverlust, desto größer die Verbesserung (Kaukua, 2004). Allerdings halten manche Verbesserungen nur flüchtig an. Verbesserungen in den psychosozialen Bereichen sind kurzlebig, während Verbesserungen der physikalischen Funktionsfähigkeit, Mobilität und Vitalität länger andauern (Seidell \& Tijhuis, 2002).

Neben den oben genannten Maßnahmen zur Gewichtsreduktion können auch andere Faktoren, wie die Teilnahme an Interventionsprogrammen zu einem Abbau der Komorbiditäten oder zur Steigerung der körperlichen Aktivität, die gesundheitsbezogene Lebensqualität verbessern (Fontaine \& Bartlett, 2003).

Chirurgische Verfahren führen zu einer merklichen und nachhaltigen Gewichtsreduktion und damit zu einer Verbesserung der Lebensqualität (Fontaine \& Bartlett, 
2003). Die Effekte einer Gewichtsreduktion durch Pharmakotherapie auf die Lebensqualität sind weitgehend unbekannt (Wirth, 2003).

\subsection{Psychopathologische Aspekte}

Frühere Studien fanden interessanterweise wenig signifikante Unterschiede bzgl. der Psychopathologie adipöser Menschen im Vergleich zu Normalgewichtigen (Wadden \& Phelan, 2002). Studien neueren Datums benutzen ein anspruchvolleres Untersuchungsdesign, haben zum Vergleich Kontrollprobanden, verwenden standardisierte Fragebögen und/oder Interviews und unterscheiden zwischen adipösen Menschen mit oder ohne Anliegen einer Gewichtsreduktion (Friedman \& Brownell, 2002; Herpertz \& Senf, 2003).

Diese Studien zeigen, dass das Übergewicht bei Frauen mit einem hohen Risiko für Major Depression, Suizidversuche und Suizidgedanken assoziiert ist. Bei Männern hingegen verringern sich durch Übergewicht das Risiko einer Depression und die Suizidalität.

Ein erhöhtes Niveau an Depression und anderer Psychopathologie ist bei adipösen Männern und Frauen mit dem Anliegen einer Gewichtsreduktionsbehandlung deutlicher als bei Normalgewichtigen oder Adipösen ohne Wunsch nach Gewichtsreduktion (Wadden \& Phelan, 2002; Herpertz \& Senf, 2003).

Auch Untersuchungen an klinischen Populationen, wie die von Fitzgibbon, Stolley und Kirschenbaum (1993) zeigen, dass adipöse Personen mit einem Therapieanliegen höhere Raten an Psychopathologie aufweisen als adipöse Personen in der allgemeinen Bevölkerung.

Bedeutungsvoller emotionaler Disstress, d.h. Symptome einer Depression oder Angst, sind wahrscheinlich Faktoren, die Menschen veranlassen professionelle Hilfe zu suchen.

Zusätzlich zu den affektiven Störungen scheint es, dass die Patienten im klinischen Stichproben wahrscheinlich häufiger als adipöse Personen in der allgemeinen Bevölkerung unter einer Essstörung im Sinne einer „Binge Eating Disorder“ (BED) oder einer deutlichen Unzufriedenheit mit dem Körperbild (body image dissatisfaction) leiden (Wadden et al., 2004; Friedman \& Brownell, 2002).

Eine Gewichtsreduktion führt in der Regel $\mathrm{zu}$ einer Besserung psychischer Symptome, insbesondere der Angst und Depressivität. Untersuchungen aus der 
Adipositaschirurgie bieten zurzeit die besten Hinweise. Die Adipositaschirurgie bei extrem adipösen Menschen (BMI $\left.>40 \mathrm{~kg} / \mathrm{m}^{2}\right)$ reduziert das Körpergewicht drastisch und bessert fast alle psychosozialen Parameter. Diese Effekte sind noch Jahre nach der Operation $\mathrm{zu}$ beobachten und dienen als Hinweis dafür, dass psychische Morbidität assoziiert mit Adipositas wahrscheinlich eher eine Folge als eine Ursache von Adipositas ist (Kaukua, 2004).

Die Effekte einer Gewichtsreduktion auf die Stigmatisierung und Diskriminierung sind weitgehend unbekannt.

\subsubsection{Adipositas und Binge Eating Disorder (BED)}

Bereits 1959 wurde von Albert Stunkard auffälliges Essverhalten bei übergewichtigen Personen beschrieben. In seiner seitdem häufig zitierten Arbeit „Eating Patterns and Obesity“ beschreibt Stunkard Essanfälle übergewichtiger Personen als ,eating, in a discrete periode of time . ., an amount of food that is definitely larger than most individuals would eat under similar circumstances“ (American Psychiatric Assosiation [APA], 1994, zitiert nach Stunkard, 2004).

Die Binge Eating Disorder (BED) oder Störung mit Essanfällen wurde 1997 als vorläufige psychiatrische Diagnose in das Diagnostische Statistische Manual psychischer Störungen (DSM-IV) aufgenommen.

BED als eine Essstörung wird vergleichbar mit der Bulimia Nervosa durch das Auftreten regelmäßiger Essanfälle charakterisiert. Ein Essanfall liegt vor, wenn innerhalb eines umschriebenen Zeitraums mehr gegessen wird, als andere Personen in einem ähnlichen Zustand und unter ähnlichen Bedingungen $\mathrm{zu}$ sich nehmen würden, und wenn die Nahrungsaufnahme mit einem Gefühl von Kontrollverlust einhergeht (de Zwaan, 2002; Stunkard, 2004).

Ein weiteres Diagnosekriterium ist eine Häufigkeit der Heißhungerattacken von mindestens 2-mal in der Woche über einen Zeitraum von 6 Monaten.

Im Unterschied zur Bulimia nervosa treten jedoch keine Ausgleichsmaßnahmen zur Gegensteuerung einer Gewichtszunahme (z. B. durch selbstinduziertes Erbrechen, die Einnahme von Laxantien/Diuretika oder Sport) in Verbindung mit den Essanfällen auf. Die Kriterien für die Diagnose einer BED sind in o.g. Weise festgelegt, werden gegenwärtig jedoch noch kontrovers diskutiert. In der International 
Classification of Diseases (ICD-10) wird die BED unter F50.9, als ,nicht näher bezeichnete Essstörungen“" vermerkt (WHO, 2006).

Obwohl die BED nicht nur bei Adipösen vorkommt, sind im klinischen Alltag die meisten Betroffenen übergewichtig. Die Prävalenz der BED gemessen anhand von Selbsteinschätzung beträgt bei übergewichtigen Personen, die an Gewichtsreduktionsprogrammen teilnehmen, um die 30 \% (Wadden, et al., 2004; de Zwaan, 2002). Wird BED von Experten diagnostiziert, erscheint die Prävalenz 10-15 \% niedriger zu sein. In der Allgemeinbevölkerung wurde eine Prävalenz von $2 \%$ gefunden. Diese Prävalenzunterschiede legen nahe, dass die von BED Betroffenen behandlungsmotivierter sind als Adipöse ohne BED (Wadden et al., 2004).

Nach de Zwaan sind Frauen etwa 1,5-mal häufiger betroffen als Männer (de Zwaan, 2002; Stunkard, 2004).

Innerhalb der Population der Adipösen mit dem Anliegen einer Gewichtsreduktion scheint es, dass die Binge Eating Disorder (BED) zu einer Erhöhung der Depressionsraten führt. Etliche Studien zeigten, dass Patienten mit einer schweren BED von einer größeren depressiven Symptomatik berichteten als Adipöse mit einer moderaten oder gar ohne BED (Wadden et al., 2004). Die überwältigende Mehrheit der Forschung auf diesem Gebiet zeigt, dass im Vergleich zu den adipösen Personen ohne BED adipöse Personen mit BED nicht nur erhöhte Symptome einer Depression, sondern auch ein niedriges Selbstwertgefühl, mehrere Symptome einer Borderline Persönlichkeitsstörung und eine signifikant größere lebenslange Prävalenz jeglicher psychischer Störungen der Achse $\mathrm{I}^{1}$ aufweisen; den Abusus von Substanzen oder Abhängigkeitserkrankungen mit eingeschlossen (Wadden et al., 2004; Stunkard, 2004).

Die Befunde hinsichtlich BED machen deutlich, wie wichtig es ist, zwischen adipösen Personen mit oder ohne Essstörung zu unterscheiden.

Die Mehrheit der adipösen Menschen leidet nicht unter einer Essstörung. Sie hat somit im Wesentlichen eine normale psychische Funktionsfähigkeit. Psychische Einschränkungen bei Adipositas finden sich in klinischen Stichproben und bei Menschen, die gleichzeitig an einer BED erkrankt sind. Adipöse Personen die unter

1 DSM IV- Achse I: Hauptsächlich Zustandsstörungen, schwere mentale Fehlstörungen und Lernunfähigkeiten (Beispiele: Schizophrenie, Depression, Angststörungen, Störungen der Impulskontrolle, Essstörungen) 
eine Essstörung leiden, berichten vermehrt von psychischen Komplikationen unabhängig davon, ob diese Personen in klinischen Settings oder in der allgemeinen Bevölkerung anzutreffen sind (Wadden et al., 2004).

Die Beziehung zwischen Adipositas, Binge Eating und Depression ist noch nicht vollständig verstanden. Aus einer klinischen Sicht ist BED ein zuverlässiges Merkzeichen für Symptome einer Depression und weist auf das Erfordernis einer genauen Abklärung der Achse I - Störungen hin (Wadden et al., 2004).

\subsection{Körperbild und Selbstkonzept bei Menschen mit Adipositas}

Das Körperbild (body image) kann als ein Teil des Selbstkonzepts aufgefasst werden, welches sich bei verschiedenen Altersgruppen und je nach Geschlecht unterschiedlich stark auf das Selbstwertgefühl auswirken kann.

Das Körperbild wird auch als Teilbereich des Körpererlebens/Körpererfahrung definiert (Petermann \& Winkel, 2003; Bielefeld, 1991).

Bielefeld (1991) unternimmt den Versuch, die Fülle der Begriffe im Bereich der Körpererfahrung auf zwei sich wechselseitig beeinflussenden Schienen zu sortieren. Nach dieser Unterteilung des Gesamtkomplexes Körpererfahrung auf den perzeptivkognitiven Zweig einerseits und emotional-affektiven Zweig andererseits. Der Begriff des Körperbildes wird dem emotional-affektiven Zweig zugeordnet (Bielefeld, 1991). 


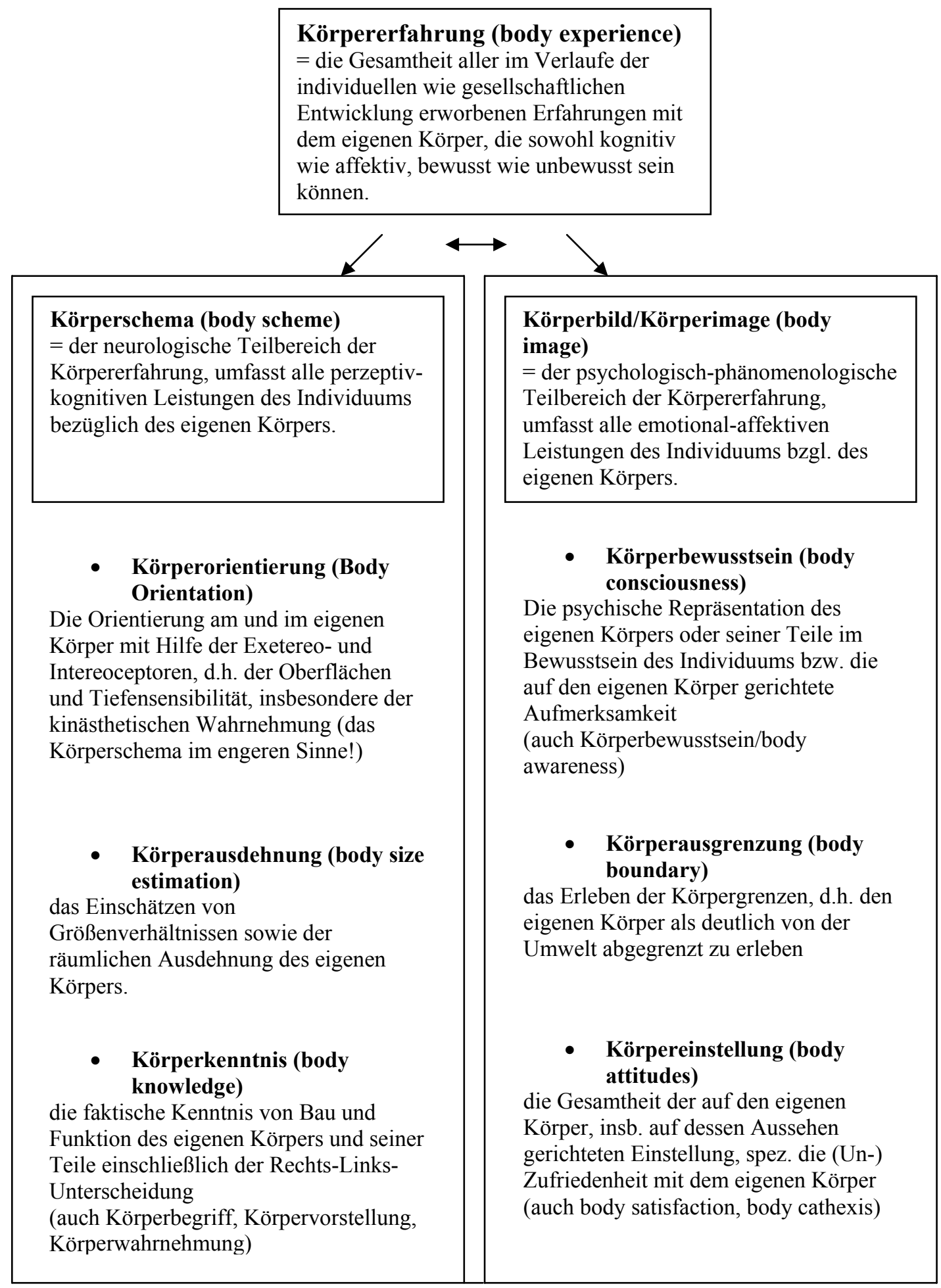

Abbildung 4: Versuch einer Strukturierung des Gesamtkomplexes Körpererfahrung (nach Bielefeld 1991, 17) 
Körperbild (Body Image) wird als der psychologisch-phänomenologische Teilbereich der Körpererfahrung definiert, der alle emotional-affektiven Leistungen des Individuums bzgl. des eigenen Körpers umfasst (Bielefeld, 1991).

Dieser emotionale Zweig der Körperwahrnehmung ist nach Petermann und Winkel (2003) aus therapeutischer Sicht relevanter als die perzeptuelle Wahrnehmung. Nach Petermann sind Störungen auf der perzeptuellen Ebene der Körperwahrnehmung sehr stabil, kaum durch therapeutische Interventionen beeinflussbar und das Wohlbefinden wenig beeinflussend.

Das Körperbild, welches die affektiven Einstellungen zum Körper umfasst, beeinflusst das emotionale Wohlbefinden hingegen stark und ist durch therapeutische Maßnahmen veränderbar (Petermann \& Winkel, 2003). Deusinger (1998) favorisiert in ihrem körperbezogenen Fragebogen zum Selbstkonzept den Begriff Körperkonzept. In diesem Kontext können die Begriffe Körperimage und Körperkonzept durchaus synonym verwandt werden.

In welcher Weise die Adipositas die Selbstwahrnehmung der Betroffenen, insbesondere das Körperbild als wichtigen Aspekt des Selbstkonzepts bestimmt, ist ein therapierelevanter Aspekt der Adipositasforschung.

Schon Stunkard und Mendelson (1967) berichten, dass adipöse Personen unter einer Körperbildstörung leiden. Diese Körperbildstörung wird nicht durchgängig bei allen adipösen Menschen betrachtet. Eher betroffen sind Personen, die schon als Kind adipös waren. Sie zeigen eine emotionale Störung und sind nach den Autoren durch ihr engeres familiäres Umfeld (Eltern, Geschwister) und gleichaltrige Freunde negativen Beurteilungen ausgesetzt (Stunkard \& Mendelson, 1967, zitiert nach Sarwer \& Thompson, 2004).

Überraschenderweise wurden diese anfänglichen Befunde über Jahrzehnte nicht weiter verfolgt. Erst ab 1990 wurde dem Thema der Unzufriedenheit mit dem äußeren Erscheinungsbild bei Menschen mit Adipositas wieder Aufmerksamkeit in der empirischen Forschung geschenkt.

Die aktuellen Forschungsarbeiten in diesem Gebiet konzentrieren sich auf die Prävalenz, die Spezifität, den Schweregrad und die klinische Signifikanz der Unzufriedenheit mit dem eigenen Körperbild.

In einer Gesellschaft, in der Dünnsein hoch geschätzt wird, ist es nicht überraschend, dass übergewichtige und adipöse Personen von einer erhöhten Unzufriedenheit mit ihrer körperlichen Erscheinung berichten. 
Genauso wenig überrascht es, dass sich die Forschung mehr auf die weibliche als auf die männliche Körperbildunzufriedenheit konzentriert. Der gesellschaftliche Druck bezüglich der äußeren Erscheinung ist für Frauen nach wie vor stärker als für Männer, obwohl sich der geschlechtsspezifische Unterschied zu verringern scheint (Sarwer \& Thompson, 2004; Johnson, 2004; Rosen, 2002).

Die Zusammenhänge zwischen Adipositas und Unzufriedenheit mit dem eigenen Körper konnten vielfach nachgewiesen worden.

Eine Körperbildunzufriedenheit (Body Image Dissatisfaction) ist weit häufiger bei Menschen mit Adipositas als eine Binge Eating Störung. Junge Menschen und Frauen scheinen besonders gefährdet zu sein (Wadden et al., 2004).

Es ist jedoch eine Tatsache, dass eine Unzufriedenheit mit dem äußeren Erscheinungsbild auch dann besteht, wenn Frauen und Mädchen normalgewichtig sind. Wie Forschungsergebnisse der letzten 10 Jahre aus vielen verschiedenen Ländern zeigen, ist das Phänomen des negativen Körperbildes und des Körpergewichts ein Charakteristikum moderner Gesellschaften.

Das Phänomen der Unzufriedenheit mit dem eigenen Körper ist so verbreitet, dass es auch als „normative Unzufriedenheit“ (normative discontent) beschrieben wird, und es ist heute so aktuell wie noch nie zuvor (Rodin, Silberstein \& Striegel-Moore, 1985, zitiert nach Sarwer \& Thompson, 2004).

Der Grad der Unzufriedenheit mit dem eigenen Körper scheint nach Forschungsergebnissen in keiner Beziehung zu dem Ausmaß des Übergewichts zu stehen (Sarwer \& Thompson, 2004).

Diese fehlende Beziehung zwischen dem negativen Körperbild und dem Grad der Adipositas unterstützt Theorien über das Körperbild, die besagen, es bestünde generell eine schwache Beziehung zwischen dem, was jemand über seinen Körper denkt und fühlt, und der objektiven Realität seiner Erscheinung.

Es wird jedoch ein Reizschwelleneffekt beschrieben. Demnach setzt eine Körperunzufriedenheit ab einem gewissen Grad der Gewichtszunahme manchmal schon im Bereich des Übergewichts ein. Diese Unzufriedenheit scheint sich aber mit dem Grad der Adipositas nicht weiter zu steigern (Cash, 1990; Sarwer, Wadden, Pertschuk \& Whitaker, 1998, zitiert nach Sarwer \& Thompson, 2004).

Sarwer \& Thompson (2004) empfehlen Vorsicht bei der Interpretation solcher Ergebnisse und heben die negativen Einflüsse der Körperbildunzufriedenheit auf das Verhalten hervor. 
Ein signifikant höherer Prozentsatz adipöser Frauen als nicht adipöser Frauen berichtet, dass sich die negative Einstellung zum Körper auf der sozialen Ebene manifestiert. Sie verstecken ihren Körper in weiter Kleidung, ändern ihre Körperhaltung oder Motorik, vermeiden es ihren Körper anzuschauen und sind emotional belastet, wenn sie über ihre Erscheinung nachdenken.

Ein ähnlich großer Prozentsatz adipöser Frauen berichtet von Gefühlen der sozialen Unsicherheit, wie Scham, Peinlichkeits- und Verlegenheitsgefühlen, aufgrund ihres Körpergewichts in alltäglichen sozialen Situationen wie bei der Arbeit oder auf Festen (Sarwer, Wadden \& Foster, 1998).

Körpergewicht scheint weiterhin auch andere profunde Verhaltensweisen zu beeinflussen. So fand Garner (1997) im Body Image Survey, dass ungefähr 50 \% der Frauen und $30 \%$ der Männer rauchen und weiter rauchen wollten, um dadurch ihr Gewicht zu kontrollieren. Mehrere Frauen in der Studie berichteten, dass ihre Besorgnis bzgl. einer Gewichtszunahme ihre Entscheidung, Kinder zu bekommen, beeinflusse.

Die beeindruckendste aller Aussagen war, dass $24 \%$ der Frauen und $17 \%$ der Männer mehr als 3 Jahre ihres Lebens aufgeben würden, um ihr Wunschgewicht zu erreichen (Garner, 1997).

Sarwer \& Thompson (2004) betonen, dass die Erfassung der Zufriedenheit mit dem Gewicht und der Körperform ein angemessenes Screeninginstrument für Menschen mit Adipositas sein könne. Die Ergebnisse solch einer Messung verschafften einen vielseitigen Eindruck des Körperbildes eines adipösen Menschen. Bei der Auswahl des Instruments empfiehlt es sich, das Geschlecht sowie die ethnische bzw. kulturelle Zugehörigkeit des Untersuchten zu berücksichtigen.

Die pathologische Bedeutung des negativen Körperbildes ergibt sich aus den Verbindungen zu anderen psychischen Symptomen und Erkrankungen.

Studien konnten den Zusammenhang eines negativen Körperbildes mit einer klinisch signifikanten Dysphorie bei adipösen Frauen nachweisen.

Studien an adipösen Frauen mit einem Therapieanliegen fanden einen Zusammenhang zwischen einem negativen Körperbild einerseits und einem niedrigen Selbstwertgefühl und mit einer erhöhten depressiven Symptomatik andererseits (Sarwer \& Thompson, 2004). So berichten in der Untersuchung von Sarwer et al. (1998) adipöse Frauen von einer signifikant höheren Unzufriedenheit mit ihrer 
körperlichen Erscheinung als die Kontrollgruppe der normalgewichtigen Frauen. Ein Drittel bis die Hälfte dieser adipösen Frauen ist so unzufrieden mit ihrer Körperform und ihrer äußeren Erscheinung, dass ihr soziales Leben beeinträchtigt wurde. Diese Unzufriedenheit und die damit verbundenen Folgen werden von den Forschern als ein subklinisches Syndrom bezeichnet. Es wird durch regelmäßige negative Äußerungen über die eigene Person, Vermeidung gesellschaftlicher Aktivitäten und durch das Verstecken von Körperteilen durch sehr weite Kleidung charakterisiert.

Eine kleine Minderheit adipöser Menschen mit einem Therapieanliegen leidet wahrscheinlich unter einem so deutlich negativen Körperbild, dass es mit einer klinisch signifikanten Depression oder ähnlichen Komplikationen assoziiert wird.

Stunkard und Mendelson (1961) beschreiben schon eine Gruppe von Patienten mit einem negativen Körperbild, die glaubten ihre Körper wären „hässlich“ und „verabscheuungswürdig“ und angaben, ihnen würde von ihren Mitmenschen mit „Feindseligkeit, Ablehnung und Missachtung“ begegnet.

„Ihr gestörtes Körperbild nimmt die Form einer überwältigenden Besorgnis über die eigene Fettleibigkeit an, welche oftmals andere persönliche Merkmale ausblendet.

Es kann z.B. keine Rolle mehr spielen, ob eine Person auch talentiert, wohlhabend oder intelligent ist. Ihr Körpergewicht ist das einzige, was sie interessiert, und sie sieht die ganze Welt unter dem Aspekt des Körpergewichts“ (Stunkard \& Mendelson, 1961, S. 328, zitiert nach Wadden et al., 2004).

Sarwer \& Thompson (2004) fassen zusammen, dass bei adipösen Menschen das Ausmaß der Adipositas nicht den Schweregrad der Unzufriedenheit mit dem eigenen Körper beeinflusst; und somit auch nicht das Ausmaß der psychischen Symptome.

Nach Sarwer et al. (1998) gibt es jedoch eine kleine Minderheit (8 \%) von adipösen Frauen, die unter einem extrem negativen Körperbild leiden, welches signifikant psychopathologisch ist. Das Ausmaß eines derart negativen Körperbildes erfüllt die diagnostischen Kriterien einer Körperdysmorphen Störung nach DSM IV (300.7, F45.2). 


\subsubsection{Körperbild und Motivation zur Gewichtsabnahme}

Weltweit werden für Produkte zur Gewichtsabnahme Milliarden von Dollar ausgegeben. Die Mehrheit der Menschen tut dieses, um ihre körperliche Erscheinung $\mathrm{zu}$ verbessern. Auch extrem adipöse Menschen, die unter erheblichen mit dem Übergewicht assoziierten Gesundheitsproblemen (wie Hypertonie, Diabetes und Osteoarthritis) leiden, sowie die Personen mit dem Anliegen eines bariatrischchirurgischen Eingriffs haben als primäres Ziel nicht die Verbesserung ihrer Gesundheit. Die Hauptmotivation für den Gewichtsverlust ist der Wunsch die körperliche Erscheinung zu verbessern (Sarwer \& Thompson, 2004).

Obwohl ein negatives Körperbild bei der Mehrheit der Betroffenen nicht mit einer signifikanten klinischen Psychopathologie einhergeht, scheint die Einstellung zum eigenen Körper eine bedeutende Rolle bei der Motivation zur Gewichtsabnahme zu spielen.

Eine Gewichtsabnahme ist vielleicht die wirkungsvollste und am weitesten verbreitete Therapie gegen ein negatives Körperbild. Nach dem aktuellen Stand der Forschung sind dauerhafte Gewichtsabnahmen nur zu einem Prozentsatz von ca. $5 \%$ möglich. Demnach sind größere Veränderungen des Körperbildes nicht realistisch, so dass andere Behandlungsmethoden zur Behandlung der Körperbildstörung nötig sind (Forster \& Wadden, 2002; Rosen, 2002).

\subsubsection{Die Behandlung der Unzufriedenheit mit dem Körperbild bei Menschen mit Adipositas}

Die therapeutischen Interventionen einer Körperbildunzufriedenheit bei Menschen mit Adipositas befinden sich noch in den Anfängen.

Obwohl Störungen im Körperbild seit langem als hauptsächlich Besorgnis erregende psychologische Faktoren für Menschen mit Adipositas bekannt sind, fingen Forscher und Therapeuten erst in den letzten Jahren an, zu erkennen, wie nötig die Integration einer systematischen Evaluation dieser Komponente in die Interventionsprogramme ist (Thompson \& Tantleff-Dunn, 1998; Sarwer \& Thompson, 2004).

Bis 1990 gibt es in der Literatur keine Studie zu psychologischen Interventionen bei Adipositas, welche vorrangig auf die Behandlung des Körperbildes abzielen. Dies ist einerseits überraschend, wenn man bedenkt, dass Stunkard und Mendelson schon 1967 die körperbezogene Unzufriedenheit bei Menschen mit Adipositas feststellten. 
Andererseits erklärt die Tatsache, dass Adipositas und ihre Behandlung üblicherweise unter physiologischen Gesichtspunkten gesehen wird, während die Körperbildtherapie psychotherapeutisch oder körpertherapeutisch gesehen wird, dass das Körperbild unterrepräsentiert ist.

Die Rolle einer Körperbildbehandlung im Rahmen der Adipositasbehandlung ist noch nicht völlig bestimmt (Sarwer \& Thompson, 2004). Untersuchungen zeigten, dass das Körperbild sich nach einer Gewichtsreduktion verbessert, aber es gibt auch Publikationen, die eine Verbesserung des Körpergefühls ohne einen Gewichtsverlust belegen. Meist handelt es sich dann um psychotherapeutisch orientierte Interventionen, die zur Behandlung des Körperbildes Verfahren der Kognitiven Verhaltenstherapie, wie Selbstmonitoring, Desensibilisierung etc. anwenden (Rosen, 2003; Sarwer \& Thompson, 2004). Cooper und Fairburn (2004) schlagen vor, die Therapie des Körperbildes der Gewichtsreduktion folgen zu lassen. Ziel sei es, in der Stabilisierungsphase einer Behandlung das langfristige Halten des Gewichts zu unterstützen. Nach den beiden Forschern scheitern traditionelle Gewichtsreduktionsprogramme häufig daran, dass sie kognitive Prozesse, die Erwartungen zur Verbesserung des Körperbildes eingeschlossen, nicht ausreichend berücksichtigen. Aus dem Grund sollen Aufrechterhaltungsprogramme auf die Änderung der Gedanken und Verhaltensweisen bzgl. des Körperbildes zielen. Sie sollen dadurch den Patienten helfen die realistischen Veränderungen wertzuschätzen.

Die beiden Forscher stellen die Hypothese auf, dass eine Behandlung des Körperbildes im Rahmen der Gewichtsstabilisierungsphase der entscheidende Mechanismus für eine erfolgreiche langfristige Aufrechterhaltung des Gewichts sein kann (Cooper \& Fairburn, 2004).

Nach Sarwer \& Thompson (2004) gibt es eine anwachsende Anzahl von Ansätzen, die Menschen helfen ihr Körperbild zu verbessern. Sie sind von Änderung des Körpergewichts oder der Körperform unabhängig. Ihre endgültige Nützlichkeit für Menschen mit Adipositas ist allerdings noch nicht bestimmt.

Kritisch zu bemerken ist, dass die Körperbildtherapie bei Patienten mit starken medizinischen Problemen nicht das vorrangige Therapieziel sein sollte. Aus gesundheitspolitischer Sicht ist die Gewichtsabnahme vorrangig, da sie in direktem Zusammenhang mit der Mortalität und Morbidität bei übergewichtigen oder adipösen Menschen steht. 
Wenn führende Forscher trotzdem die Einbeziehung der Körperbildbehandlung als eine Komponente eines Gewichtsprogramms fordern, liegt es daran, dass Forschungsergebnisse zeigen:

- Eine körperbezogene Unzufriedenheit führt zu einer Wiederzunahme an Gewicht.

- Biologische Faktoren machen es praktisch unmöglich, die aktuellen kulturellen Erscheinungsbildideale zu erreichen

- Der einzige bleibende psychologische Unterschied zwischen adipösen und normalgewichtigen Menschen kann auf dem Gebiet des Körperbildes bestehen (Thompson, 2003, S. 6).

Darüber hinaus stellt Thompson die Hypothese auf, dass Menschen, die eine Körperbildtherapie bekommen, auch bei realistischen Gewichtsverlusten eine positive Veränderung in Bezug auf ihr Selbstbild erfahren und so motivierter an weiteren Gewichtsveränderungen arbeiten als Menschen, die keine deutlichen Effekte der Gewichtsabnahme auf die Selbstwahrnehmung spüren (Sarwer \& Thompson, 2004).

In der Bewegungstherapie wird die verbesserte Körperakzeptanz oft als Ziel der bewegungstherapeutischen Inhalte angegeben, ist für Adipöse aber nicht wissenschaftlich belegt. Ob die verbesserte Selbstakzeptanz als ein automatisches Produkt der Bewegung oder über bestimmte Prozesse und eine gezielte Methodik erreicht werden soll, bleibt offen. 


\section{Therapieansätze bei Adipositas}

Unter der Überschrift „Adipositastherapie“ wurde in den letzten 30 Jahren eine beinahe zahllose Vielfalt von Methoden und Maßnahmen entwickelt.

Daraus entstanden für Prävention und Therapie eine Vielzahl von Konzepten, die sich zum einem in ihrer wissenschaftlichen Basis, zum anderen in ihrer Struktur-, Prozess- und Ergebnisqualität, vor allem aber in ihren Inhalten unterscheiden.

Obwohl durch einige Behandlungen eine signifikante kurzfristige Gewichtsreduktion möglich ist, bleibt die Gewichtsabnahme von einem optimalen Maß entfernt, und es ist schwierig sie langfristig aufrechtzuerhalten.

Adipositasbehandlung ist mehr und mehr Gegenstand von genauen Untersuchungen und Kritik geworden. Es werden zunehmend Qualitätsanforderungen an die Therapieansätze gestellt, und es kommt dazu, dass die Betroffenen wachsam gegenüber unseriösen Angeboten werden und die Fachleute in ihrer Arbeit vermehrt auf Effektivität achten (Grillo, 2003).

Nach Ellrott (2003) erfolgt die medizinische Behandlung von Übergewicht und Adipositas beispielsweise im Vergleich zur koronaren Herzerkrankung wenig differenziert. Noch fehlen die Prädiktoren für den zu erwartenden individuellen Therapieerfolg in einem standardisierten Behandlungskonzept. Individualisierte Therapiekonzepte werden benötigt und stehen noch in der Anfangsphase (Ellrott, 2003).

Das wichtigste Kriterium für die Therapieindikation und die Auswahl eines Therapieverfahrens ist bislang der Body Mass Index (BMI) sowie vorhandene Komorbiditäten oder durch das Übergewicht entstehende Risiken für Gesundheitskomplikationen.

Nach den Leitlinien der Deutsche Adipositas Gesellschaft (2006) ist grundsätzlich eine Adipositasbehandlung ab einem BMI von $\geq 30 \mathrm{~kg} / \mathrm{m}^{2}$ indiziert. Bei Übergewicht mit einem BMI zwischen 25 und 29,9 ist eine therapeutische Intervention angezeigt, wenn zusätzlich zu dem Übergewicht folgende Merkmale vorliegen:

- übergewichtsbedingte Gesundheitsstörungen (z.B. Hypertonie, Typ-2 Diabetes) oder

- abdominale Fettverteilungsmuster oder

- Erkrankungen, die durch Übergewicht verschlimmert werden oder

- hoher psychosozialer Leidensdruck (DAG, 2006). 
Es ist ersichtlich, dass die angezeigte Therapiestrategie stark vom BMI des Patienten abhängt. Auch in Deutschland sind die differentialtherapeutischen Empfehlungen in einem Flussdiagramm „Adipositasprävention und -therapie“ (siehe Anhang) von der Deutschen Adipositas- Gesellschaft (DAG) nach dem BMI zusammengefasst.

„Je höher der Ausgangs-BMI, desto höher das Risiko für Komorbiditäten und desto größer wird der notwendige therapeutische Aufwand (Kosten/Jahr); desto geringer ist aber die therapeutische Verfügbarkeit“ (Ellrott, 2003, S. 186).

Die Therapieempfehlungen in den Vereinigten Staaten sind den deutschen sehr ähnlich (siehe Anhang). Das „National Heart, Lung, and Blood Institute (NHLBI) und die „North American Association for the Study of Obesity“ (NAASO) veröffentlichten 2000 in dem Bericht „Practical Guide to the Identification, Evaluation and Treatment of Overweight and Obesity in Adults" u.a. die aktuellen therapeutischen Richtlinien für die amerikanische Bevölkerung (NHLBI \& NAASO, 2000).

Sehr charakteristisch für beide Therapieempfehlungen ist das so genannte „Basisprogramm“ zum Gewichtsmanagement. Es handelt sich um einen multimodalen Interventionsansatz, welcher aus drei verschiedenen Behandlungsformen: Ernährungs-, Verhaltens- und Bewegungstherapie, besteht und konstant bei jeder Adipositasstufe an erster Stelle empfohlen wird (DAG, 2006; NHLBI \& NAASO, 2000; Lehrke \& Laessle, 2003).

Anzumerken ist auch, dass körperliche Aktivität als eine feste therapeutische Intervention von größter Bedeutung anerkannt ist. Sie ist unerlässlich für jede Adipositasbehandlung. Dabei geht es nicht nur um die Gewichtsreduktion oder Gewichtsstabilisierung, sondern auch um die Auswirkungen auf Komorbiditäten anderer Erkrankungen und Gesundheitsrisiken.

Die verschiedenen Ansätze ergänzen sich gegenseitig, so dass durch eine Kombination der verschiedenen Verfahren eine Optimierung des Therapieerfolges erreicht werden kann.

Durch diese Dreifachkombination lassen sich die größten und stabilsten Therapieeffekte erzielen (NHLBI \& NAASO, 2000, Lehrke \& Laessle, 2003).

Als oberste Prämisse gilt, den Patienten individuell der für ihn vermeidlich bestmöglichen Behandlungsform zuzuweisen. Aus diesem Grund sollen neben dem medizinischen Zustand (BMI-Wert, Risikofaktoren, Komorbiditäten) auch die Motivation und Bereitschaft des Patienten erfragt und berücksichtigt werden (Weinsier \& Kushner, 2002; NHLBI, 1998). 
Der Behandlungsplan soll den Vorstellungen und Lebensgewohnheiten des Patienten entsprechen. Der Patient soll in die Therapieplanung mit einbezogen werden.

Im Folgenden werden die wichtigsten, gängigsten derzeit zur Verfügung stehenden ernährungswissenschaftlichen, somatisch-medizinischen und verhaltenstherapeutischen Therapiemethoden vorgestellt.

Die medizinische Behandlung wurde ausgewählt, weil sie einen hohen Stellenwert hat, und weil Ärzte häufig die erste Anlaufstelle für Menschen sind, die etwas gegen ihr Übergewicht unternehmen wollen. Außerdem legen Ärzte von sich aus vielen ihrer Patienten im Hinblick auf Abwendung einer Gesundheitsgefährdung durch Übergewicht eine Gewichtsreduktion nahe.

Die Verhaltenstherapie wird dargestellt, weil ihr die gängigen psychologischen Therapiemethoden bei Adipositas zuzuordnen sind und sie im Konzept dieser Arbeit von größerer Bedeutung ist als tiefenpsychologisch orientierte Konzepte. In der Literatur ist die Verhaltenstherapie bei Adipositas häufig untersucht, über die Anwendung psychoanalytisch orientierter Ansätze bei der Adipositasbehandlung wird dagegen meist nur in Form von Kasuistiken berichtet (Tuschhoff, BeneckeTimp \& Vogel, 1995).

Da die Bewegungs- und Sporttherapie im Mittelpunkt der Untersuchung steht, wird diese anschließend ausführlich und in einem gesonderten Kapitel behandelt. 


\subsection{Ernährungstherapie}

Die Ernährungstherapie wird von Expertengremien aller bisherigen Therapierichtlinien als Eckpfeiler des Langzeitgewichtsmanagements adipöser Patienten bezeichnet (DAG, 2006). Die Ernährungstherapie (Diätetische Maßnahmen) ist weit verbreitet und wird in unterschiedlichen Formen ausgeführt. Sie umfasst verschiedene Stufen bzw. Strategien. Der Einstieg ist auf jeder Stufe möglich. Nach Abschätzung des individuellen Risikoprofils sowie Berücksichtigung der individuellen Gegebenheiten wird mit der geeigneten Form begonnen (DAG, 2006).

Das Behandlungsprinzip der Ernährungstherapie beruht auf einer Reduktion der Energieaufnahme. Dadurch kann eine wirksame negative Energiebilanz und somit eine Gewichtsabnahme erzielt werden.

Von großer Bedeutung ist nach einer initialen Gewichtsreduktion die Gewichtsstabilisierung bzw. die Minimierung einer erneuten Gewichtszunahme. Dieses kann nur durch eine langfristige Ernährungsumstellung erreicht werden und setzt eine hohe Compliance seitens des Patienten voraus.

Hinsichtlich der Ernährungstherapie werden von der Deutschen Adipositas Gesellschaft folgende Grundsätze empfohlen:

- In der Praxis sollte bei der Ernährungsumstellung das gesamte Umfeld des Patienten einbezogen werden, um die Kurz- und Langzeitcompliance zu verbessern.

- Der Patient muss über die Prinzipien der Ernährungsumstellung gut informiert sein.

- Ein tägliches Energiedefizit von 500-1000 kcal sollte angestrebt werden, um einen Gewichtsverlust von $0,5-1,0 \mathrm{~kg} /$ Woche und insgesamt von $10 \%$ des Ausgangsgewichts zu erzielen (Lauterbach et al., 1998; DAG, 2006).

Dieses Energiedefizit soll vor allem durch eine Reduktion der Nahrungsfette erreicht werden. Diese sollten auf etwa $30 \%$ der Gesamtkalorien begrenzt werden. Die Deutsche Gesellschaft für Ernährung (DGE) und die Deutsche Adipositas Gesellschaft postulieren eine energiereduzierte, fettarme, kohlenhydrat- und ballaststoffreiche Ernährung mit einer Nährstoffrelation von 15-20 \% Proteine, 25-30 \% Fette und 50-60 \% Kohlenhydrate (Lauterbach et al., 1998; DAG, 2006).

Es gibt eine Reihe von Diäten (Blitz-, Crash- und Hungerdiäten), die nicht als ernst zu nehmende Therapien in Frage kommen (Ellrott \& Pudel, 1998). Nach den Richtlinien der Deutschen Adipositas Gesellschaft sind solche Außenseiterdiäten aus 
unterschiedlichen Gründen (fehlende wissenschaftliche Belege, hohe medizinische Risiken) gefährlich, ungeeignet und nicht zu empfehlen (DAG, 2006).

Derzeit lassen sich die therapeutisch relevanten diätetischen Maßnahmen grob in zwei Kategorien einteilen: die fettarme Mischkost (ohne Kalorienbegrenzung) und die kalorienreduzierte Mischkost (Diäten mit sehr niedriger Kalorienzufuhr).

Das Prinzip der fettarmen Mischkost beruht auf der Begrenzung der Fettaufnahme auf etwa 60-80 g/tgl. (entspricht in etwa den DGE- Empfehlungen zur täglichen Fettzufuhr). Die Kalorienberechungen (Abwiegen, Protokollieren) für die Nahrungsaufnahme durch den Betroffenen entfällt und macht die praktische Umsetzung leichter. Bei der Behandlungsmethode geht es ausschließlich um die Beschränkung der Fettaufnahme. Da viele epidemiologische Studien in den letzten Jahren einen positiven Zusammenhang zwischen Fettverzehr und Körpergewicht belegen, wurde verstärkt empfohlen nur die Fettaufnahme zu reduzieren, die Kohlenhydrataufnahme aber ,ab libitum“ zu erlauben (Wilhelm, Strütt-Neeb, Opielka \& Cuntz, 2002; DAG, 2006).

Bei der kalorienreduzierten Mischkost wird die tägliche Kalorienaufnahme je nach Alter, Körpergröße und Gewicht reduziert. Verschiedene dieser Diäten sind die niedrig kalorische Kost (Low Caloric Diet LCD, 700-1000 kal/Tag) und die extrem niedrig kalorische Kost (Very Low Caloric Diet VLCD, 450-700 kal/Tag). LCD und VLCD sind drastisch kalorienreduzierte Ernährungsformen und werden meist als Formuladiäten durchgeführt. Es handelt sich um Nahrungssubstrate, die nach einer ernährungsphysiologischen Formel zusammengesetzt sind. Meistens werden sie in Form von Nährstoff- Drinks (Milchshakes) oder einer drastisch energiereduzierten meist proteinreichen Kost (Riegel) eingenommen und induzieren eine rasche Gewichtsabnahme. Diese Diäten scheinen aber für eine langfristige Gewichtskontrolle wenig geeignet zu sein. Aufgrund des erheblichen Nährstoffdefizits sollen sie nur für eine begrenzte Zeit (2 bis maximal 12 Wochen) angewendet werden. Wegen des schnellen initialen Gewichtsverlustes sind solche Diätprogramme nur bei stark adipösen Patienten indiziert, die aus medizinischen Gründen (z.B. präoperative Phase) kurzfristig Gewicht verlieren sollen.

Formuladiäten sollten im Rahmen einer umfassenden Adipositastherapie angewandt werden. Sie sind nur in Kombination mit einem umfassenden Verhaltenstraining mit 
der Zielsetzung, Ernährungs- und Bewegungsgewohnheiten langfristig zu verändern, sinnvoll (Lauterbach et al., 1998; Lehrke \& Laessle, 2003).

Bei einer längeren Anwendung solcher drastischen Diätprogramme treten nicht unerhebliche Nebenwirkungen auf. Aus diesem Grund sollten sie besonders qualifizierten Therapeuten vorbehalten bleiben oder nur in entsprechenden Spezialeinrichtungen eingesetzt werden (Lauterbach et al., 1998; Lehrke \& Laessle, 2003).

Ein weiterer Kritikpunkt ist, dass bei allen rein diätetischen Ansätzen situative, soziale, emotionale und sensorische Einflussfaktoren auf das Konsum- und Verzehrverhalten weitgehend ignoriert werden.

Des Weiteren sind Erfolge in kurzfristigen Gewichtsreduktionsphasen unter Alltagsbedingungen selten aufrechtzuerhalten. In der langfristigen Aufrechterhaltung von Therapieerfolgen zeigen sich allerdings erst Qualität und Effektivität des Behandlungsansatzes. Nur selten werden während der diätetischen Gewichtsreduktionsphase auch Strategien zur Gewichtserhaltung besprochen oder eingeübt (Wilhelm et al., 2002).

\subsection{Medikamentöse Therapie}

Bisherige Ansätze zur medikamentösen Adipositastherapie sind mit hohen Nebenwirkungsraten, übermäßigem Gebrauch oder Missbrauch der Medikamente sowie Rückfällen assoziiert.

Neuere Medikamente, die über die Inhibition von Magen- und Pankreaslipasen wirken, erzielten zunächst vielversprechende Ergebnisse und machten weniger abhängig. Aber auch hier zeigten sich negative Folgen für die Gesundheit und es ergaben sich Rückfälle, wenn die medikamentöse Behandlung beendet wurde (Aronne, 2002).

Eine zusätzliche Pharmakotherapie zur Gewichtssenkung ist dann zu empfehlen, wenn:

- die Behandlung der Adipositas mit dem Basisprogramm (Lebensstiländerung) langfristig eine geringe Erfolgsrate hat;

- sich mit zunehmender Dauer und dem Schweregrad der Adipositas Krankheiten summieren (Wirth, 2003). 
Die genauen Voraussetzungen für eine medikamentös begleitete Therapieindikation werden gemäß der „National Task Force on the Prevention and Treatment of Obesity“ 1996 formuliert (DAG, 2006).

Die Pharmakotherapie soll zwei Ziele haben: Zum einen soll sie kurzfristig die Gewichtsabnahme begünstigen und zum anderen zur langfristigen Gewichtsstabilisierung beitragen. So wird medikamentöse Therapie als eine adjuvante sowie additive Maßnahme im Rahmen eines umfassenden Therapiekonzepts, bestehend aus diätetischen und verhaltenstherapeutischen Maßnahmen, befürwortet (NHLBI \& NAASO, 2005; DAG, 2006). Ein alleiniger medikamentöser Ansatz zur Adipositastherapie kann zum heutigen Zeitpunkt nicht als sinnvoll angesehen werden. Die Pharmakotherapie ersetzt weder eine Ernährungsumstellung noch eine Bewegungstherapie, da sie nur solange erfolgreich ist, wie das Medikament verabreicht wird (Lehrke \& Laessle, 2003).

Derzeit stehen zwei Gruppen von Medikamenten zur Verfügung:

- die im Zentralnervensystem wirksamen Substanzen, die als Appetitzügler bzw. Sättigungsverstärker wirken und

- die in der Peripherie wirksamen Assimilationshemmer.

Entsprechend dieser zwei o.g. Gruppen sind zurzeit zwei gewichtssenkende Substanzen (Antiadiposita) zugelassen und sollen daher im Folgenden kurz beschrieben werden.

\section{Sibutramin:}

Die Substanz gehört der Gruppe der zentralnervös wirkenden Medikamente an. Bei Sibutramin handelt es sich um einen selektiven Serotonin- und NoradrenalinWiederaufnahmehemmer. Sibutramin bewirkt eine vorzeitige Beendigung der Nahrungsaufnahme, es verlängert nicht die Abstände zwischen den Mahlzeiten. Die Substanz wirkt als ein Sättigungsverstärker und nicht als ein Appetithemmer, zusätzlich stimuliert sie den Energieverbrauch. So hat Sibutramin einen dualen Wirkmechanismus, es reduziert die Energieaufnahme und stimuliert den Energieverbrauch (Wirth, 2003).

Die wichtigsten Nebenwirkungen sind Mundtrockenheit, Obstipation, Schwindel, Schlafstörungen, außerdem Anstieg der Blutdruckwerte und der Herzfrequenz von 35 Schlägen/min. Wichtige Kontraindikationen sind Hypertonie, Koronare Herzkrankheit, Glaukom und Herzrhythmusstörungen (DAG, 2006). 


\section{Orlistat:}

Orlistat ist ein Inhibitor von Magen- und Pankreaslipasen, die eine dominierende Rolle in der Fettverdauung spielen. Orlistat wirkt fast ausschließlich im Gastrointestinaltrakt und hemmt dosisabhängig die Fettabsorption bis zu $30 \%$ und senkt dadurch das Körpergewicht.

Die wichtigsten Nebenwirkungen beschränken sich auf das Gastrointestinalsystem. Vermehrtes Auftreten weicher Stühle, Anstieg der Defäkationsfrequenz, Flatulenz, und Steatorrhoe sind zu nennen (Wirth, 2003). Zwischen 5 und $15 \%$ der Patienten zeigen eine verminderte Absorption fettlöslicher Vitamine, deren klinische Bedeutung ungeklärt ist (DAG, 2006).

Die Deutsche Adipositas Gesellschaft bleibt bzgl. der Pharmakotherapie kritisch und empfiehlt von einer längeren Anwendung abzusehen, da bisher Erfahrungen zum klinischen Einsatz der beiden Substanzen nur für eine begrenzte Dauer von 2 bis 4 Jahren vorliegen. Weiterhin fehlen für die beiden Wirkstoffe prospektive Studien zu kardiovaskulären Risiken. Mögliche Nebenwirkungen wie eine negative Beeinflussung von adipositasassoziierten Krankheiten sollten bei dem Einsatz der Medikamente mit berücksichtigt werden.

Der Nutzen einer Kombination von Orlistat mit Sibutramin könnte nur theoretisch von Vorteil sein, denn er ist derzeit noch nicht ausreichend belegt (Toplak, 2003; DAG, 2006).

Es gibt eine Reihe anderer Medikamente (wie z.B. Diuretika, Amphetamine, Dexamphetamin und Thyroxin) mit gewichtssenkendem Potential. Diese kommen aus unterschiedlichen Gründen - Wirkungslosigkeit oder gefährliche Nebenwirkungen - für die Adipositasbehandlung nicht in Frage und sollten daher in Gewichtsmanagementprogrammen nicht verwendet werden (DAG, 2005).

\subsection{Chirurgische Therapie}

Bariatrische Chirurgie setzt sich als Methode zur Behandlung massiven Übergewichts $\left(B M I \geq 40 \mathrm{~kg} / \mathrm{m}^{2}\right)$ in den letzten Jahren zunehmend durch. Neue operative Methoden mit geringerem Komplikationsrisiko entwickelten sich zu einer maßgeblichen und einzig langzeitig effektiven Therapie für die extreme Adipositas. 
Chirurgische Eingriffe gewannen vermehrt die Akzeptanz der Mediziner und der Öffentlichkeit (Latifi, Kellum, De Maria \& Sugerman, 2004; Pories \& Beshay, 2002; Wirth, 2003).

Kriterien für die Indikation eines bariatrisch-chirurgischen Eingriffs sind in den Leitlinien der Deutschen Adipositas Gesellschaft (2006) definiert und stimmen mit den internationalen Kriterien überein:

- Der Patient sollte einen BMI $\geq 40$ oder 35 mit erheblichen Komorbiditäten bzw. Risikofaktoren haben.

- Die konservativen Behandlungsmöglichkeiten unter professioneller Aufsicht waren langfristig ohne Erfolg.

- Das Operationsrisiko darf nicht inakzeptabel hoch sein. Die Nutzen - RisikoAbwägung muss eindeutig positiv sein.

- Die Patienten müssen ausreichend motiviert und vollständig aufgeklärt sein sowie ihr Einverständnis gegeben haben (DAG, 2006).

Um das Operationsrisiko gering zu halten, sollten die Kliniken spezialisiert sein und möglichst das ganze Spektrum der operativen adipositasspezifischen Techniken anbieten sowie Rezidiveingriffe durchführen. Der laparoskopische Zugang wird, wenn immer möglich, empfohlen, damit die Patienten weniger Belastung durch die Operation erfahren. Aus einer Reihe verschiedener Techniken zur chirurgischen Adipositasbehandlung werden aktuell vor allem drei Verfahren angewandt (Kielmann \& Herpertz, 2002).

\subsubsection{Restriktive Verfahren}

Restriktive Methoden sind zurzeit die gängigen Methoden und arbeiten nach dem Prinzip, die Menge der aufgenommenen Nahrung zu vermindern. Gastric Banding (GB), Vertikal Banded Gastroplasty (VBG), Silastic Ring Vertical Gastroplasty $(\mathrm{SRVG})$ stellen einen Vormagen (Pouch = Magentasche) her, der nur eine geringe Nahrungsaufnahme (15-30 ml) pro Mahlzeit erlaubt. Nur durch eine Öffnung (Stoma) von $12 \mathrm{~mm}$ bleibt der Vormagen mit dem Restmagen verbunden. Der Nahrungsbrei muss diese kleine Öffnung zur weiteren Verdauung passieren. Durch diese Maßnahme wird bereits nach geringer Nahrungszufuhr ein Sättigungsgefühl 
vermittelt. Auf diesem Wege kann aber nur die Menge der Nahrung und nicht der Kaloriengehalt kontrolliert werden (Husemann 1997; Pories \& Beshay, 2002).

Derzeit werden die Eingriffe am Magen bevorzugt. Sie haben ein geringes Risiko und lassen sich, wie das anpassbare Magenband, auch laparoskopisch durchführen. Damit ist die Erholungszeit des Patienten wesentlich kürzer als bei Laparotomie (Bauchschnitt). Die Rate an unmittelbaren postoperativen Komplikationen ist außerordentlich gering (Latifi et al., 2004).

\subsubsection{Magen-Bypass (Gastric-Bypass) und kombinierte Verfahren}

Das Magen-Bypass-Verfahren ist heute mit Abstand die meist ausgeführte Operation in den Vereinigten Staaten. Es wird in der bariatrischen Chirurgie wegen der niedrigen Mortalitätsrate und der exzellenten Ergebnisse als „Gold Standard“ akzeptiert (Pories \& Beshay, 2002). Da zur Durchführung dieser Operation der Bauchraum in Vollnarkose eröffnet werden muss, wurde die laparoskopische Rouxen-Y-Gastric-Bypass-Operation (Magenbypass) entwickelt.

Der Magen-Bypass kann auch als eine Kombination aus restriktivem und malabsorptivem Verfahren operiert werden. $\mathrm{Zu}$ diesen kombinierten Verfahren zählen Biliopancreatic Diversion (BPD) und Distal Gastric Bypass (DGBP) (Kielmann \& Herpertz, 2002). Bei diesen Operationen wird durch eine Klammernaht ein $50 \mathrm{ml}$ fassender Magenteil vom Restmagen getrennt. Dieser Magenanteil wird mit einer Dünndarmschlinge (Jejunum-Schlinge) verbunden. Wie viel Dünndarm von der Verdauung ausgeschaltet wird, kann operativ festgelegt werden. Die Nahrung passiert nach der Speiseröhre den kleinen neu gebildeten Magen und fließt direkt in den verkürzten Dünndarm. So wird die Malabsorption der verringerten Nahrungsmenge erreicht (Wirth, 2003).

Diesen Verfahren ist gemeinsam, dass unterschiedliche Dünndarmteile, größer als beim Magen-Bypass funktionell ausgeschaltet werden. Die Länge des funktionierenden Dünndarmes entscheidet über das Ausmaß der Malabsorption, die im Vergleich zum Magen-Bypass erheblich höher ist (DAG, 2006).

Er wird in der Regel erst bei Patienten ab einem BMI von $50 \mathrm{~kg} / \mathrm{m}^{2}$ angewandt oder wenn restriktive Verfahren bei einem Patienten keinen Erfolg brachten oder aufgrund der Aufnahme kalorienreicher Getränke oder Süßwaren nicht sinnvoll angewandt werden können (DAG, 2006). 
Hinsichtlich der Gewichtsabnahme sind die kombinierten Verfahren die effektivsten bariatrischen Methoden (Pories \& Beshay, 2002).

\subsubsection{Plastisch-chirurgische Verfahren}

Plastisch-chirurgische Verfahren wie Liposuktion oder Dermolipektomie sind keine Verfahren, um generalisierte Adipositas zu behandeln.

Fettabsaugung (Liposuktion) kann zur Entfernung überschüssiger lokaler Fettdepots eingesetzt werden. Sie hat eine rein kosmetische Indikation und ist somit zur Behandlung von Adipositas nicht geeignet. „Ein Nutzen dieser Technik für die langfristige Gewichtssenkung ist nicht belegt, die Risiken dieses Eingriffs sind schlecht dokumentiert, aber nicht unerheblich“ (DAG, 2006, S. 18).

Dermolipektomie kann nach erfolgreicher Gewichtsabnahme erforderlich sein, um überschüssige Haut zu entfernen und dabei das Risiko für intertriginöse Hautinfektionen zu beseitigen (Husemann, 1998; Wirth, 2003).

\subsubsection{Zusammenfassende Bewertung chirurgischer Verfahren}

Operative Methoden sind bezüglich der Gewichtsabnahme allgemein sehr erfolgreich.

Das Ausmaß des zu erwartenden Gewichtsverlustes nach chirurgischer Adipositasbehandlung hängt von der gewählten Operationsform ab. Der Langzeit- Gewichtsverlust beim Gastric Bypass und den kombinierten Verfahren ist höher als der bei den rein restriktiven Verfahren (DAG, 2006; Miller \& Hell, 2002).

Nach bariatrischen Eingriffen wird der Gewichtsverlust erleichtert und gewährleistet eine Besserung und Heilung der zahlreichen Komorbiditäten. Dadurch wird aber nicht die Adipositas als chronische Krankheit mit multifaktorieller Genese geheilt. Eine monotherapeutische Sichtweise auch im Rahmen der Adipositaschirurgie greift zu kurz. Bei der Adipositaschirurgie kommt insbesondere der Nachsorge eine wichtige Bedeutung zu. Alle bariatrisch-chirurgischen Methoden erfordern eine konsequente Nachbehandlung der Patienten um Komplikationen zu vermeiden bzw. diese zu erkennen. Für einen Langzeiterfolg muss die Nachbehandlung in der Regel über mehrere Jahre erfolgen. Aus diesem Grund sollten die Patienten in ein lang- 
jähriges interdisziplinäres Therapiekonzept integriert werden (DAG 2006; Lehrke \& Laessle, 2003; Kielmann \& Herpertz 2002).

\subsection{Verhaltenstherapeutische Verfahren}

Verhaltenstherapeutische Strategien zur Adipositasbehandlung werden seit den 60er Jahren angewandt. Die Ziele eines verhaltenstherapeutischen Treatments zur Gewichtsreduktion (Behavioral Weight Loss (BWL) Treatment) sind primär die Modifikation der Essgewohnheiten und des Ausmaßes an körperlicher Aktivität der betroffenen Personen. Der Schwerpunkt bei der Verhaltensänderung liegt in einer kalorien- reduzierten Nahrungsaufnahme und in einer Erhöhung des Energieverbrauchs durch körperliche Aktivität. Dies führt zu einer negativen Energiebilanz und in Konsequenz dieser $\mathrm{zu}$ einer Gewichtsreduktion. Verhaltenstherapeutische Methoden sind intensiver erforscht und ihre Effekte sind gründlicher dokumentiert als jeder andere therapeutische Ansatz der Adipositastherapie. Behandlungsprinzipien und Methoden sind in ,anwenderfreundlichen“ Behandlungsmanualen dargestellt. Dies hat zur Folge, dass verhaltenstherapeutische Behandlungstechniken weitgehend verbreitet und akzeptiert sind. Die verhaltenstherapeutische Intervention ist eine unerlässliche Komponente jeder angemessenen modernen Adipositasbehandlung (Wilson \& Brownell, 2002; Lehrke \& Laessle, 2003).

Betrachtet man den Einsatz psychotherapeutischer Maßnahmen genau und detailliert, so lassen sich grundsätzlich zwei Zielrichtungen unterscheiden:

„Zum einen die direkte Behandlung der Adipositas, d. h. die Gewichtsreduktion soll unmittelbar durch psychologische Methoden herbeigeführt werden. Zum anderen die indirekte Behandlung der Adipositas, die bei den angenommenen psychischen (Mit-) Auslösern der Störung, aufrechterhaltenden Bedingungen oder Folgeproblemen ansetzt. Dadurch sollen dann mittelbar positive Effekte auch bei der Gewichtsreduktion erzielt oder zumindest erleichtert werden “ (Margraf, 2002).

Allerdings wird hier von Margraf (2002) kritisch angemerkt, dass bisher valide empirische Belege für die „,indirekte“ psychotherapeutische Gewichtsreduktion weitgehend fehlen. Psychotherapeutische Verfahren ohne konkrete Ziele im Ernährungs-, Gewichts- und Körperaktivitätsbereich kommen also als Behandlungsmethode erster Wahl nicht in Frage. Symptomferne psychotherapeutische Verfahren haben ihre 
Bedeutung eher bei der Bewältigung von psychischen Folgeproblemen der Adipositas. Diese sind vor allem bei jungen Frauen stark ausgeprägt (vgl. Kap. 2.9). Nach dem aktuellen Forschungsstand haben sich bei der direkten psychotherapeutischen Behandlung der Adipositas kognitive und verhaltenstherapeutische Methoden im Hinblick auf allgemeine Wirksamkeit am besten bewährt. Zentrale Aspekte der Adipositas wie Essen, Trinken und Bewegung sind auf der Verhaltensebene angesiedelt, so dass eine direkte verhaltenstherapeutische Intervention auf dieser Ebene ansetzt und plausibel erscheint.

Die Ergebnisse verhaltenstherapeutischer Behandlungsprogramme hinsichtlich der Gewichtsreduktion stimmen größtenteils überein. Es lassen sich im Durchschnitt Gewichtsreduktionen von 7-10 \% des initialen Körpergewichts über eine Behandlungszeitspanne von 16 bis zu 24 Wochen erzielen. Allerdings gilt auch für diese wie für jede andere bisher erforschte Adipositastherapie, dass nach dieser kurzfristigen Gewichtsabnahme die langfristige Stabilität der Gewichtsreduktion über mindestens fünf Jahre nicht ausreichend erreicht werden kann (Wilson \& Brownell 2002; Margraf, 2002). Neueste Studien fanden, dass Patienten im Durchschnitt um ein Drittel bis zur Hälfte ihres Gewichtsverlustes ein Jahr nach der Behandlung wieder zugenommen hatten. Dabei verläuft die Gewichtsabnahme bei den meisten Patienten kontinuierlich, so dass in 5 Jahren der gesamte Gewichtsverlust wiedererlangt wird (Wilson \& Brownell, 2002; Grillo, 2003).

Verhaltenstherapeutische Adipositasprogramme beinhalten in der Regel:

- eine ausführliche kognitive Vorbereitung und Psychoedukation der Patienten (Vermittlung von Ernährungswissen, Erklärungsmodell der Störung, realistische Ziele, Motivationsstärkung),

- eine ausführliche Verhaltens- oder Problemanalyse (auslösende und aufrechterhaltende Bedingungen, genaue Beschreibung des Problemverhaltens),

- direkte Maßnahmen zur Veränderung problematischer Verhaltensweisen (z.B. Selbstkontrolltechniken, Verhaltenstraining) und Einstellungen (z.B. kognitive Interventionen) (Margraf, 2002 ). 
Wirth (2003) benennt die Elemente eines typischen Programms zur Verhaltensmodifikation wie folgt:

1. kognitive Vorbereitung des Patienten: Eruierung von Gründen zur Gewichtsreduktion; Bedeutung von Ernährung und Bewegung im Lebenskontext; Therapieziele; Motivationsverstärkung; Gestaltung des Therapie-Settings.

2. Analyse der kognitiven, affektiven und motorischen Bedingungen zur Entstehung der Adipositas; Erfahrungen; Einstellungen; Gewohnheiten; Gefühle; Rahmenbedingungen.

3. Informationen: Ursachen und Folgen der Adipositas; Ernährungsphysiologie; Lebensmittelkunde; Kochkunde; sportmedizinische Grundlagen; Bewegungsarten.

4. Vermittlung von praktischen Fähigkeiten: Einkaufen; Kochen; Sport; Argumentationshilfen gegenüber Angehörigen, Partnern, Arbeitskollegen.

5. Implementierung von Reizkontrolltechniken; Einkaufsliste; Essensplanung; EssArrangement; Einladungen; Urlaub; Sondersituationen.

6. Kognitive Umstrukturierung; negative Gedanken durch positive ersetzen; Imperative wie „nie und „immer“ vermeiden; Orientierung an realistischen Zielen und Erfolgen.

7. Verstärkung des neuen Verhaltens: Protokolle für Gewicht, Ernährung und Bewegung; Belohnungen für erreichte Ziele; Verstärkerrolle der Gruppe, von Angehörigen, dem Partner.

8. Rückfallprophylaxe: Identifizierung risikobehafteter Situationen; Erarbeitung von Problemlösungsstrategien; Bearbeiten von Versagens- und Schuldgefühlen.

Abbildung 5: Elemente eines typischen Programms zur Verhaltensmodifikation (nach Wirth, 2003, 132) 


\subsubsection{Prinzipien der Verhaltenstherapie bei Adipositas}

Die wichtigsten Prinzipien verhaltenstherapeutischer Interventionen bei Adipositas basieren auf dem Effekt des klassischen Konditionierens und weisen einen ziel- und prozessgerichteten Charakter auf (Munsch \& Margraf, 2003).

$\mathrm{Zu}$ Beginn der verhaltenstherapeutischen Behandlung werden durch eine Verhaltensanalyse ungünstige Verhaltensmuster, die zur Entstehung des Übergewichts beigetragen haben, bezüglich ihrer auslösenden und aufrechterhaltenden Bedingungen analysiert (Lehrke \& Laessle, 2003).

Die adipösen Patienten formulieren daraufhin Ziele und Zwischenziele. In Form eines Selbstmanagementtrainings werden Techniken vermittelt, um diese Ziele zu erreichen. Gelingt es nicht ein neues Verhalten im Alltag zu implementieren, so werden Hindernisse identifiziert und das Vorgehen daraufhin angepasst. Als Zielvorgaben werden jeweils kleine Veränderungsschritte angestrebt. Eine realistische Zielsetzung wie die Unterteilung in kleine überschaubare Teilziele, macht es möglich, dass schnelle Erfolge erlebt werden, so dass die Motivation gefördert wird. Erfolge wirken verstärkend und daher verhaltensstabilisierend, wohingegen Misserfolge das Verhalten destabilisieren. Das Fördern günstigen und der Abbau ungünstigen Verhaltens basiert auf den allgemeinen Gesetzmäßigkeiten von Verhaltensänderungen (Munsch \& Margraf, 2003).

Der Patient soll in die Lage versetzt werden, seine Umwelt so umzugestalten, dass er die neuen Verhaltensweisen langfristig durchhalten kann, so dass die zunächst notwendige therapeutische Kontrolle allmählich in eine Selbstkontrolle des Patienten übergeht (Lehrke \& Laessle, 2003).

Es muss betont werden, dass die Selbstmanagement - Therapie im Hinblick auf Adipositastherapie von wesentlicher Bedeutung ist (Kanfer, Reinecker \& Schmelzer, 2006). Die Patienten müssen ein Vorgehen lernen, mit dem sie sich ein Leben lang in Bezug auf ihr Gewicht helfen können (Benecke, 2002).

In der folgenden Tabelle werden die verhaltenstherapeutischen Prinzipien, die verhaltenstherapeutischen Interventionen sowie deren Einsatzbereich und Effektivität dargestellt: 
Tabelle 7: Interventionen und verhaltenstherapeutische Prinzipien in der Adipositasbehandlung (nach Munsch \& Margraf, 2002, 226)

\begin{tabular}{|c|c|c|c|}
\hline Interventionen & Prinzipien & Einsatzbereich & Wirksamkeitsnachweis \\
\hline $\begin{array}{l}\text { Selbst- } \\
\text { beobachtung }\end{array}$ & $\begin{array}{l}\text { - Erfassung und Analyse } \\
\text { reaktionskontingenter } \\
\text { Bedingungen } \\
\text { - Unterbrechen } \\
\text { automatischen Verhaltens } \\
\rightarrow \text { Operantes Lernen }\end{array}$ & $\begin{array}{l}\text { Ess-und } \\
\text { Ernährungs- } \\
\text { Verhalten } \\
\text { Bewegungs- } \\
\text { verhalten }\end{array}$ & $\begin{array}{l}\text { Selbstbeobachtung korreliert } \\
\text { mit langfristiger } \\
\text { Gewichtsreduktion } \\
\text { (Wadden, 1995; Baker, 1993; } \\
\text { Guare et al., 1989; Cooper \& } \\
\text { Fairburn 2001) }\end{array}$ \\
\hline Stimuluskontrolle & $\begin{array}{l}\text { - Veränderung der } \\
\text { Auslösereize, Abbau } \\
\text { problematischen/Aufbau } \\
\text { erwünschten Verhaltens } \\
\rightarrow \text { Klassisches } \\
\text { Konditionieren, Extriktion, } \\
\text { Operantes Lernen } \\
\end{array}$ & $\begin{array}{l}\text { Essverhalten } \\
\text { Bewegungs- } \\
\text { verhalten }\end{array}$ & $\begin{array}{l}\text { Verbesserung der } \\
\text { Selbstkontrolle des } \\
\text { Essverhaltens durch externe } \\
\text { Stimuluskontrolle } \\
\text { (Ferster, 1962) }\end{array}$ \\
\hline $\begin{array}{l}\text { Motivationale } \\
\text { Strategien } \\
\text {-Selbstver- } \\
\text { stärkung }\end{array}$ & $\begin{array}{l}\text { - Darbieten eines } \\
\text { offenen/verdeckten Stimulus } \\
\text { als Konsequenz eines } \\
\text { Verhaltens, zwecks } \\
\text { Frequenzsteigerung } \\
\text { erwünschten Verhaltens } \\
\rightarrow \text { Operantes Lernen, } \\
\text { Selbstregulation }\end{array}$ & $\begin{array}{l}\text { Selbstbeobachtung, } \\
\text { Ess- und } \\
\text { Ernährungs- } \\
\text { verhalten } \\
\text { Bewegungs- } \\
\text { verhalten }\end{array}$ & $\begin{array}{l}\text { Selbstverstärkung fördert } \\
\text { langfristiges Bemühen um } \\
\text { Zielerreichung (Mitchell, } \\
\text { 1980; Flanery \& } \\
\text { Kirschenbaum, 1986) }\end{array}$ \\
\hline -Selbstbestrafung & $\begin{array}{l}\text { - Senkung der zukünftigen } \\
\text { Auftretenswahrscheinlichkeit } \\
\text { unerwünschten Verhaltens } \\
\rightarrow \text { Operantes Lernen, } \\
\text { Selbstregulation }\end{array}$ & $\begin{array}{l}\text { Bestrafung als } \\
\text { diskriminativer } \\
\text { Reiz für alter- } \\
\text { natives Verhalten }\end{array}$ & $\begin{array}{l}\text { Selbstbestrafung unterstützt } \\
\text { in Zusammenhang mit } \\
\text { Selbstverstärkung die } \\
\text { Gewichtsreduktion } \\
\text { (Mahoney, Moura \& Wade, } \\
\text { 1973; Agathon, 1976) }\end{array}$ \\
\hline $\begin{array}{l}\text {-Externe } \\
\text { Verstärker }\end{array}$ & $\begin{array}{l}\text {-Fremdverstärkung } \\
\text { erwünschten Verhaltens } \\
\rightarrow \text { Operantes Lernen }\end{array}$ & $\begin{array}{l}\text { Unterstützen } \\
\text { gewichtsredu- } \\
\text { zierender } \\
\text { Verhaltensweisen } \\
\text { Motivations- } \\
\text { steigerung }\end{array}$ & $\begin{array}{l}\text { Kein Effekt z.B. mittels } \\
\text { finanzieller Anreize (Jeffrey } \\
\text { et al., 1993; Jeffrey, Wing, } \\
\text { Thorson \& Burton, 1998) }\end{array}$ \\
\hline $\begin{array}{l}\text {-Soziale } \\
\text { Unterstützung }\end{array}$ & $\begin{array}{l}\text {-Motivationssteigerung, } \\
\text { Verbesserung der Selbst- } \\
\text { akzeptanz, fördern sozialer } \\
\text { Fertigkeiten } \\
\rightarrow \text { Operantes Lernen, } \\
\text { Transfer in den Alltag, } \\
\text { Schaffen vermehrter } \\
\text { diskriminativer Reize für } \\
\text { Verhaltensänderung }\end{array}$ & $\begin{array}{l}\text { Soziale } \\
\text { Unterstützung bei } \\
\text { gewichts- } \\
\text { reduzierenden Ver- } \\
\text { haltensweisen }\end{array}$ & $\begin{array}{l}\text { Soziale Unterstützung fördert } \\
\text { den Gewichtsverlust und } \\
\text { dessen Stabilisierung (Wing } \\
\text { \& Jeffrey, 1999; Foreyt \& } \\
\text { Goodrick, 1991) }\end{array}$ \\
\hline
\end{tabular}


Fortsetzung Tabelle 7: Interventionen und verhaltenstherapeutische Prinzipien in der Adipositasbehandlung (nach Munsch \& Margraf, 2002, 226)

\begin{tabular}{|c|c|c|c|}
\hline Kognitive & & & \\
\hline $\begin{array}{l}\text { - Realistische } \\
\text { Gewichtsziele }\end{array}$ & $\begin{array}{l}\text { - Verändern unrealistischer } \\
\text { Erwartungen, } \\
\text { Rückfallprophylaxe } \\
\rightarrow \text { Kognitive Umstrukturierung, } \\
\text { Steigerung der } \\
\text { Selbstwirksamkeit }\end{array}$ & $\begin{array}{l}\text { Realistische } \\
\text { Zielsetzungen, } \\
\text { Verbessern der } \\
\text { Motivationslage }\end{array}$ & $\begin{array}{l}\text { Training im Setzen } \\
\text { realistischer Gewichtsziele } \\
\text { verbessert Zufriedenheit mit } \\
\text { Gewichtsreduktion } \\
\text { (Smith, 1999) }\end{array}$ \\
\hline $\begin{array}{l}\text {-Kognitive Ver- } \\
\text { haltenssteuerung }\end{array}$ & $\begin{array}{l}\text { - Unerwünschte Gedanken } \\
\text { und Verhaltensweisen steuern } \\
\rightarrow \text { Kognitive Kontrolle }\end{array}$ & $\begin{array}{l}\text { Flexible } \\
\text { Kontrolle des } \\
\text { Essverhaltens }\end{array}$ & $\begin{array}{l}\text { Training in flexibler } \\
\text { Kontrolle korreliert mit } \\
\text { niedrigerem BMI und } \\
\text { erhöhtem Gewichtsverlust } \\
\text { (Westenhoefer, Stunkard\& } \\
\text { Pudel, 1999) }\end{array}$ \\
\hline $\begin{array}{l}\text { Rückfall- } \\
\text { prophylaxe } \\
\text { - Stressmanage- } \\
\text { ment }\end{array}$ & $\begin{array}{l}\text { - Entspannungsmethoden als } \\
\text { alternative Reaktionen auf } \\
\text { Stressoren } \\
\rightarrow \text { Psychophysiologische } \\
\text { Spannungsreduktion fördert } \\
\text { Inkompatibles Verhalten }\end{array}$ & $\begin{array}{l}\text { Bewältigung von } \\
\text { Risikosituationen }\end{array}$ & $\begin{array}{l}\text { Stressmanagement } \\
\text { verbessert langfristige } \\
\text { Stabilisation des reduzierten } \\
\text { Gewichts (Foreyt, 1994; } \\
\text { Foreyt \& Poston, 1998) }\end{array}$ \\
\hline $\begin{array}{l}\text { - Rückfall- } \\
\text { prophylaxe }\end{array}$ & $\begin{array}{l}\text { - Antizipieren vom Risiko- } \\
\text { situationen und deren } \\
\text { Bewältigung } \\
\rightarrow \text { Konfrontation in sensu, } \\
\text { antizipierte Bewältigung } \\
\text { verbessert } \\
\text { Selbstwirksamkeitseinschätzung }\end{array}$ & $\begin{array}{l}\text { Verhindern und } \\
\text { Bewältigen von } \\
\text { Risikosituationen }\end{array}$ & $\begin{array}{l}\text { Isoliertes Training in } \\
\text { Rückfallprophylaxe wenig } \\
\text { effektiv, Wirkung nur in } \\
\text { Kombination mit sozialer } \\
\text { Unterstützung (Perri, } \\
\text { Shapiro, Ludvig, } \\
\text { Twentymann \& Mc Adoo, } \\
\text { 1984; Baum, Clark \& } \\
\text { Sandler, 1991) }\end{array}$ \\
\hline
\end{tabular}

\subsubsection{Techniken der Verhaltenstherapie}

Im Folgenden werden die in der verhaltenstherapeutischen Praxis etablierten Therapietechniken kurz beschrieben.

- Selbstbeobachtung (Self-monitoring)

Die Selbstbeobachtung problematischen und unerwünschten Verhaltens stellt eine wichtige Grundlage der Verhaltenstherapie bei der Adipositas dar (Munsch \& 
Margraf, 2003; Wirth, 2000). Es bildet die Vorraussetzung für die Selbstregulation. Das Problemverhalten wird detailliert definiert und anschließend in seiner Häufigkeit, Intensität und Dauer protokolliert. Über diese Rückmeldeschleife wird erreicht, dass unerwünschtes, automatisches Verhalten bereits vor der Durchführung registriert und damit der Ausführungsprozess unterbrochen werden kann.

In der Praxis der Adipositastherapie bezieht sich die Selbstbeobachtung auf das Essund Bewegungsverhalten. Mit Hilfe systematischer "Tagebücher" protokolliert der Patient seine Nahrungsaufnahme und seine körperliche Aktivität sowie die damit einhergehenden Reaktionen, Gedanken, Anlässe, Beurteilungen etc..

- Stimuluskontrolle

Die Stimuluskontrolle als Strategie zählt zu den ersten verhaltenstherapeutischen Konzepten der Adipositasbehandlung. Mit Hilfe von Stimuluskontrolltechniken verändert eine Person ihre physikalische oder soziale Umgebung so, dass das Zielverhalten häufiger bzw. das Problemverhalten seltener auftritt (Munsch \& Margraf, 2003)

Der Einsatz von Stimuluskontrolltechniken basiert auf der Annahme, dass Übergewichtige bei ihrer Nahrungsaufnahme von Antezedenzen bestimmt werden. Es wird davon ausgegangen, dass bei Adipösen zu viele falsche Stimuli existieren, die eine erhöhte Nahrungsaufnahme zur Folge haben (www.adipositas-online.de, 2006).

Durch Kontrolle auslösender Reize sollen Gelegenheiten zum übermäßigen Essen vermieden werden. So sollte z.B. das Einkaufen nur mit vollem Magen und vorher erstellten Einkaufslisten erfolgen, Mahlzeiten sollten geplant und $\mathrm{zu}$ festgelegten Zeiten an definierten "Essensplätzen" eingenommen werden etc.

Die Stimuluskontrolle allein erweist sich zur stabilen Veränderung problematischen Verhaltens als nicht ausreichend. Es wird in der Regel in Kombination mit weiteren Methoden zur Selbstkontrolle angewandt (Munsch \& Margraf, 2003).

- Motivationale Strategien

Motivationale Strategien umfassen verhaltenstherapeutische Interventionen wie die Selbst- und Fremdverstärkung; Selbstbestrafung und die soziale Unterstützung (Munsch \& Margraf, 2003). 
Bei den Verstärkungsstrategien handelt es sich um die positive bzw. negative Verstärkung eines Verhaltens. Bei den Fremdverstärkungstechniken entscheidet der Therapeut darüber, ob verstärkt wird, während bei den Selbstverstärkungstechniken der Klient selbst bestimmt, ob er sich einen Verstärker appliziert. Sowohl über die Fremd- wie auch die Selbstverstärkung ergibt sich eine Erhöhung der Rate des erwünschten Verhaltens und eine Bereitschaft Neues zu lernen bzw. eine Verbesserung der Motivation.

Beide Strategien erweisen sich generell genauso effektiv (Munsch \& Margraf, 2003). Insbesondere sollte jedoch die Selbstverstärkung als eine bewährte verhaltenstherapeutische Technik bei der Gewichtskontrolle kurz erläutert werden. Sie stellt die letzte Phase nach der Selbstbeobachtung und Selbstbewertung zur Erhöhung der Selbstkontrolle dar. Mit gezielter Selbsteinflussnahme werden eigene Leistungen in Richtung auf das therapeutische Ziel anerkannt und selbst belohnt. Die Betroffenen lernen allmählich auf Grund angenehmer Folgen eines offenen bzw. verdeckten Stimulus, das erwünschte Verhalten häufiger zu zeigen (www.adipositas-online.de, 2006).

Im Gegensatz zur Selbstbelohnung wird bei der Selbstbestrafung als Folge eines unerwünschten Verhaltens ein leicht aversiver Reiz angewendet. Diese Selbstkontrollprozedur soll die Auftretenswahrscheinlichkeit des unerwünschten Verhaltens senken. Dieses Verfahren wurde bereits in den 70er Jahren eingesetzt und untersucht. In der Adipositasbehandlung wird heutzutage überwiegend positiv verstärkt, um ein bestimmtes Essverhalten zu erlernen bzw. seine Auftrittswahrscheinlichkeit zu erhöhen. Positive Verstärkungstechniken werden auch beim Aufbau von Bewegungsverhalten eingesetzt.

Ein weiterer wichtiger motivationaler Faktor für die langfristige Gewichtsreduktion ist soziale Unterstützung. Personen, die auf ein höheres Maß an sozialer Unterstützung zurückgreifen können, zeigen in Gewichtsreduktionsprogrammen bessere Ergebnisse. Der Begriff der sozialen Unterstützung umfasst dabei sowohl den Einschluss von Familienmitgliedern in die Behandlung als auch den Grad der Integration in ein soziales Netz. Soziale Unterstützung fördert gewichtsreduzierende Verhaltensweisen und unterstützt im Sinne einer positiven Rückmeldung an die eigene Person die Selbstakzeptanz. Sie führt zu intensiveren sozialen Kontakten und fördert die Bewältigung von psychosozialen Konflikten (Foreyt \& Poston, 1998). 
- Kognitive Techniken

Adipöse Patienten haben oft falsche, festgefahrene und irrationale Vorstellungen über die Ursachen des Übergewichts und die Möglichkeiten der Veränderung. Kognitive Techniken wie die kognitive Umstrukturierung unrealistischer Gewichtsziele machen solche Gedanken bewusster und damit einer Veränderung zugänglich (Benecke, 2002).

Die Umstrukturierung geschieht im Hinblick auf das funktionale Einsetzen hilfreicher Gedanken und das Erarbeiten von Strategien, mit deren Hilfe die Verhaltenskette, die zu ungünstigem (Ess-) Verhalten führt, frühzeitig unterbrochen werden kann.

Das Prinzip der kognitiven Kontrolle des Essverhaltens zählt zu den Kognitiven Techniken. Das Denken adipöser Personen wird häufig durch rigides kontrollmechanisches dichotomes Denken (Schwarz-Weiß-Denken) charakterisiert. Dies äußert sich im typischen Diätdenken und -verhalten, bei dem bestimmte Lebensmittel, zum Beispiel Süßigkeiten, verboten sind. In der Therapie angestrebt wird eine flexible Kontrolle, bei der es sich um eine langfristige Kontrolle der Frequenz und Quantität, mit der verschiedene Nahrungsmittel verzehrt werden, handelt (Petermann \& Häring, 2003).

- Rückfallprophylaxe

Ein Rückfall kann definiert werden als eine schrittweise Wiederzunahme an Gewicht nach initialer Gewichtsabnahme. Wenn man die Aufrechterhaltung der Gewichtsabnahme als Anwendung der Regeln eines adäquaten Essverhaltens definiert, ist ein Rückfall weiterhin charakterisierbar als die Unterbrechung der Anwendung einer oder mehrerer dieser Regeln (Marlatt, 1995).

Gegenstand eines Rückfallverhütungstrainings ist die Tatsache, dass sich früher oder später alle Patienten einmal überessen. Damit diese Ereignisse Einzelfälle bleiben und nicht zur vollständigen Aufgabe der Gewichtskontrollstrategien führen, werden spezifische Techniken benötigt, deren Vermittlung in einem Rückfallverhütungstraining stattfindet (www.adipositas-online.de, 2006; Perri, 2002).

Psychosozialer Stress ist ein Prädiktor für Rückfälle in alte automatisierte Verhaltensmuster und Gewichtszunahme. Im Stressmanagement Training werden den Patienten Entspannungsmethoden, zum Beispiel progressive Muskelrelaxation 
oder Autogenes Training, als Strategien vermittelt. In Stresssituationen sollen diese eine unmittelbare Spannungsreduktion bewirken. Diese und weitere Stressbewältigungskompetenzen verhindern, dass üblicherweise durchgeführtes, ungünstiges Verhalten (z. B. unkontrolliertes Essen) stattfinden kann (Munsch \& Margraf, 2003). Strategien zum Stressmanagement sind insbesondere zur Aufrechterhaltung der erreichten Gewichtsreduktion wirksam (Foreyt \& Poston, 1998).

Weiterhin kommen beim Training der Rückfallprophylaxe verhaltenstherapeutische (Bewältigungsplan für Risikosituationen) und kognitive Strategien (kognitive Umstrukturierung) zur Anwendung (Perri, 2002).

Ein alleiniges Rückfallverhütungstraining ohne Nachbehandlungskontakt scheint allerdings nicht effizient zu sein. Wird eine Nachsorge in Form eines therapeutischen Kontakts durchgeführt, so bringt ein Rückfallverhütungstraining sehr gute Ergebnisse (www.adipositas-online.de, 2006; Perri, 2002).

\subsubsection{Rahmenbedingungen der Verhaltenstherapie bei Adipositas}

Durchgeführt wird die verhaltenstherapeutische Adipositastherapie meist ein- bis zweimal in der Woche in Form von Gruppensitzungen (6-10 Personen) über einer Dauer von 90 Minuten. Gruppensitzungen sind kosteneffektiv und bieten soziale Unterstützung. Die Sitzungen sollten von ausgebildeten Verhaltenstherapeuten geleitet werden.

Wirth (2003) betont, dass die Gruppenleiter Kenntnisse von allen Aspekten der Adipositas, insbesondere der Ernährungs- und Bewegungstherapie besitzen sollen. Die Dauer der Behandlung schwankt zwischen einigen Wochen und einem Jahr, wobei zunehmend längere Betreuungszeiträume als günstig erachtet werden. Den größten langfristigen Erfolg haben verhaltenstherapeutische Interventionen dann, wenn sie mit anderen Maßnahmen wie fettreduzierter Ernährung, Bewegungssteigerung und langfristiger persönlicher Nachsorge kombiniert werden (Margraf, 2002). 


\subsubsection{Verhaltenstherapeutische Techniken zur Bewegungssteigerung}

Die Vermittlung einer körperlich aktiveren Lebensweise gehört zu den Hauptzielen einer Adipositasbehandlung. Diese Zielsetzung wird auch von der Verhaltenstherapie verfolgt und erfährt seit 1995 eine besondere Beachtung. Dieses liegt daran, dass das Ausmaß der körperlichen Aktivität einen nachweisbaren Prädiktor für eine langfristige Erhaltung der Gewichtsreduktion darstellt (Wing, 2004).

Im Folgenden sollen die für den Aufbau und die Erhaltung eines gesunden Bewegungsverhaltens sinnvollen verhaltenstherapeutischen Techniken im Kontext der Bewegungssteigerung erläutert werden.

Die Informationsvermittlung ist in Bezug auf die verhaltenstherapeutische Behandlung des Bewegungsverhaltens ein sinnvoller Beginn.

Denn nach Grillo et al. (1993) kann fehlendes Wissen die Ursache eines ungünstigen Verhaltens sein, wenn er sagt: „Poor health behaviors can reflect lack of knowledge as well as nonadherence“ (Grillo et al., 1993, S. 266).

Dabei handelt es sich um die Vermittlung relevanter Informationen über den Zusammenhang von Bewegungen bzw. sportlichen Aktivitäten und der Adipositas. Es ist nötig den Betroffenen auf die besondere Bedeutung der verschiedenen Bereiche, der Bewegung als Bewegungstherapie, des Gesundheitssports und der körperlichen Aktivitäten im Alltag aufmerksam $\mathrm{zu}$ machen. Da die meisten Menschen mit Adipositas unangenehme Erfahrungen im Bereich des Sports in der Vergangenheit machten, muss verhindert werden, dass diese negative Erfahrung in einem sehr speziellen Bewegungsbereich die Akzeptanz der therapeutisch relevanten Bewegungsbereiche beeinflusst. Häufige Erfahrungen aus dem Schulsport sind, als Mitspieler nicht erwünscht zu sein, bloßgestellt zu werden und schlechte Schulnoten zu erhalten. Dazu kommt, dass das Verständnis des Leistungssports, gekennzeichnet durch Vergleiche, Gewinner, Verlieren und Rangplätze, als die einzige Form sich zu bewegen gesehen wird (Grillo et al., 1993). Es ist nötig zunächst Informationen über Sportarten, grundlegende Prinzipien der Trainingslehreprinzipien (Häufigkeit, Dauer, Intensität) sowie die Rolle der Alltagsaktivitäten zu geben. Das Wissen um die Auswirkungen körperlicher Aktivitäten auf die psychophysische Gesundheit und das Übergewicht soll die Betroffenen zusätzlich motivieren und den Focus weg von einer kurzfristigen Gewichtsabnahme auf eine langfristige Umstellung des Bewegungsverhaltens hinlenken. Es sollten eigene sportliche Interessen und Bedürfnisse geweckt 
werden, die zu einer Steigerung der Lebensqualität und einen besseren Umgang mit sich selbst führen.

Die verhaltenstherapeutische Technik der Selbstbeobachtung bedeutet in Hinblick auf eine Bewegungssteigerung, dass Bewegungsprotokolle geführt werden. So ist es möglich, eine detaillierte Auflistung jeder einzelnen körperlichen Aktivität und ihrer Merkmale wie Häufigkeit, Dauer und Intensität zu erlangen. Zusätzlich können die Situationen, in denen die motorischen Handlungen stattfinden, dokumentiert sowie die damit einhergehenden Reaktionen, Gedanken, Gefühle, Anlässe, Beurteilungen etc. erfasst werden. Das Verfahren ist die Grundlage für eine Verhaltensanalyse, aus der sich eine weitere Planung der Ziele und Veränderungen ergibt. Weiterhin ist es möglich, die Einstellungen der Betroffenen gegenüber der „Bewegung“ zu erfassen.

Die Bedeutung der Dokumentation des Bewegungsverhaltens ist noch größer, wenn man berücksichtigt, dass adipöse Menschen öfters das Ausmaß körperlicher Aktivität überschätzen (Lichtman et al., 1992, zitiert nach Foreyt \& Poston, 1998).

Das Protokollieren an sich hat schon einen therapeutischen Effekt. Es hat sich gezeigt, dass die Aufzeichnung von Bewegung in selbständig durchgeführten Programmen zu einer erhöhten körperlichen Aktivität führt (King, Taylor, Haskell \& Debusk, 1988, zitiert nach www.adipositas-online.de, 2006).

Neben dem Protokollieren in Worten können auch Grafiken angefertigt werden, welche die erbrachten Leistungen visualisieren und die zusätzlich von anderen Personen (z. B. Bewegungstherapeuten) abgezeichnet werden können.

Auch die Techniken zur Stimuluskontrolle können für den Bewegungsbereich gewinnbringend eingesetzt werden (Knapp, 1988). Reize, die einer erhöhten Aktivität, also dem gewünschten Verhalten, entgegenstehen, werden vermindert. Der Patient setzt sich selbst Reize, die das gewünschte Verhalten auslösen (Brownell, 1995). Erinnerungsstücke für körperliche Bewegung können eingesetzt werden. Beispielsweise, dass die Sportbekleidung für den nächsten Tag am Abend vorher bereitgelegt wird. Die Sporttasche wird an einen sichtbaren Platz (Flur) gestellt oder mit ins Auto genommen, damit eine sportliche Aktivität nach der Arbeit unternommen werden kann. Weiterhin können kleine Erinnerungszettel innerhalb der Wohnung oder im Terminkalender hinterlassen werden. Oder ein Arbeitskollege, der am Fenster vorbeiläuft, wird zum Auslöser des täglichen Spaziergangs vor dem Mittagessen (Fox, 1992). 
Motivationale Strategien wie die Selbstverstärkung können auch in Bezug auf das Bewegungsverhalten angewandt werden. So können eigene sportliche Handlungen, die einer therapeutischen Zielsetzung zugehörig sind, anerkannt und selbst belohnt werden. „Wenn ich mein Walkingtraining absolviert habe, kaufe ich mir meine Lieblings DVD. “Es versteht sich von selbst, dass sportliche Aktivitäten nicht mit Essen belohnt werden (Knapp, 1988).

Bei der Fremdverstärkung ist die Rolle des Bewegungs- und Sporttherapeuten von Bedeutung. Er kann ohnehin während einer sportlichen Aktivität loben und die Teilnehmer individuell motivieren (Wing \& Jakicic, 2000).

Soziale Unterstützung ist eine weitere sehr wertvolle motivationale Strategie zur Steigerung, Stabilisierung und Aufrechterhaltung des Bewegungsverhaltens. Der Grad der Unterstützung durch Ehepartner, Familie und andere wichtige Bezugspersonen kann für das Bewegungsverhalten von Bedeutung sein. Grillo et al. (1993) weisen darauf hin, dass Unterstützung durch den Ehepartner mit einer verbesserten Bewegungscompliance assoziiert ist. Eine weitere Unterstützung erfährt man im Rahmen einer Sportgruppe (z.B. Verein). Hier kann die Motivation für körperliche und sportliche Betätigung über die gemeinsamen sportlichen Aktivitäten in der Gruppe entstehen. Die Wahrscheinlichkeit, dass sportliche Aktivitäten stattfinden und erhalten bleiben, ist im Gruppenverband wesentlich größer als bei individuell ausgeführten Bewegungsformen. Negativer sozialer Support („Sabotage“) bzgl. körperlicher und sportlicher Aktivitäten muss ein Thema in der Therapie sein (Benecke, 2002).

Kognitive Techniken wie die kognitive Umstrukturierung sind auch im Hinblick auf das Bewegungsverhalten sehr nützlich (Knapp, 1988). Adipöse Patienten haben auch in Bezug auf Bewegung und Sport dysfunktionale Gedanken und Meinungen über sich selbst und ihr Gewicht. Sie haben ein niedriges Selbstwertgefühl und ein negatives Körperbild.

Dysfunktionale Denkmuster bezüglich der Bewegung und sportlicher Aktivitäten können sich wie folgt äußern:

- die Einstellung zum Sport und zur Bewegung

(„Sport ist für sportliche dünne Leute gemacht.“ - „Es wird immer verglichen.“ „Sport ist mit Noten bzw. sportlichem Leistungsdruck verbunden.“) 
- die Rechtfertigung des Bewegungsverhaltens

(„Das nutzt sowieso nichts.“ - „Ich bin sowieso gleich außer Atem.“ - „Das brauche ich gar nicht erst zu probieren.")

- das Selbstbild

(„Ich habe eben nicht den nötigen starken Willen.“ - „Ich bin ein Versager, ein Turnbeutelvergesser.“)

- das Körperbild

(„Ich bin eklig.“ - „An mir ist nichts Schönes.“ - „Beim Federballspielen werde ich schwitzen und stinken.")

- das Verhalten anderer

(„Die gucken schon immer so, wenn ich gehe.“ - „Die tuscheln dann über mich und lachen mich aus, wenn ich mit den Nordic-Walking-Stöcken gehe.“)

- die Therapieziele

(„Ich will mindestens $30 \mathrm{~kg}$ abnehmen.“ - „Ich will jetzt ganz viel Sport treiben, jeden Tag eine Stunde.“ - „Wenn ich erst einmal schlank bin, dann habe ich keine Probleme mehr.“ - „Ich werde nie mehr den Fahrstuhl benutzen.“ - „Ich werde immer mit dem Fahrrad fahren.“ (Benecke, 2002).

Das Einsetzen hilfreicher Kognitionen und das Erarbeiten von Strategien sollen die Betroffenen dazu bringen, sich aktiv zu hinterfragen und ihren inneren Dialog zu ändern. Eine Selbstaussage wie „Ich bin so müde, warum muss ich es jetzt tun?“ soll zu einem „Ich weiß, ich fühle mich ganz wohl, wenn ich es gemacht habe.“ geändert werden. Dadurch sollen Kognitionen, die zu einem ungünstigen Bewegungsverhalten führen, frühzeitig unterbrochen werden.

Im Rahmen der Rückfallprophylaxe werden Stressmanagementtechniken angeboten. Es werden traditionelle Entspannungstechniken (Jacobson-Methode, Autogenes Training) vermittelt. Solche Verfahren können Inhalte bewegungstherapeutischer Interventionen sein. Auch andere bewegungs- und sporttherapeutischen Inhalte (z.B. Funktionsgymnastik) bringen neben dem Fitnesseffekt einen Entspannungseffekt mit 
sich. Auch andere körperliche Aktivitäten, wie z.B. ein Spaziergang, können spannungslösende Effekte hervorrufen.

Wie oben erwähnt, zählt die Beibehaltung der körperlichen und sportlichen Aktivitäten zu den besten Indikatoren für eine langfristige Erhaltung der Gewichtsreduktion. Sie bietet sich somit als Strategie zur Rückfallprophylaxe an.

Aber auch für die Bewegung an sich sind verhaltenstherapeutische Maßnahmen für die Vorbeugung eines Rückfalls in die Inaktivität von Bedeutung. Solche Rückfallpräventionsstrategien wurden entwickelt, um mit riskanten Situationen (z.B. Verletzungen), die sich negativ auf das Bewegungsverhalten auswirken, besser umgehen zu können.

Die Betroffenen sollen a) darin geschult sein riskante Situationen zu identifizieren, genau zu bezeichnen und evtl. vorauszusehen, b) in der Lage sein sie zu vermeiden und/oder zu bewältigen. Ihnen sollten c) die positiven Wirkungen der körperlichen Aktivitäten bewusst sein und sie sollten d) einen Plan haben für unvermeidliche Flüchtigkeitsfehler und e) über flexible Regeln für die Aufrechthaltung ihrer körperlichen Aktivitäten verfügen. f) Die körperlichen Aktivitäten sollten so ausgewählt sein, dass sie ihnen Spaß machen (Knapp, 1988).

Ein weiteres wichtiges verhaltenstherapeutisches Verfahren ist die Zielsetzung (goal setting). Es müssen zunächst auch hier im Bezug auf das Ausmaß der körperlichen Aktivitäten wie auch der Trainingsumfänge realistische, realisierbare Ziele erarbeitet werden. Es empfiehlt sich, jeweils kurz- und langfristige Ziele zu setzen. So sollte beispielsweise die Treppe am Arbeitplatz zweimal täglich für die ersten drei Wochen benutzt werden mit dem langfristigen Ziel, nur noch die Treppe zu nutzen.

Als effektiv hat sich auch eine flexible Zielsetzung erwiesen, die mit einer größeren Einhaltung des Bewegungsprogramms assoziiert ist als eine rigide Zielsetzung (Brownell, 1995). Das Walkingtraining sollte 45 min lang dauern, darf aber aus Wettergründen, Gründen der Tagesform etc. an diesem Tag auch mal 30 min dauern. Als Alternative könnte evtl. auf dem Fahrradergometer trainiert werden. Weiterhin sollen kleinere Fehlschläge (z.B. 1 Woche Sportabstinenz durch Erkältung) nicht als allgemeines Scheitern angesehen werden, welches zu einer völligen Aufgabe des Bewegungsprogramms führen könnte. 


\section{Bewegungstherapie bei Adipositas}

Ein weiterer Therapiebaustein der Adipositasbehandlung ist die Bewegungstherapie. So sollte jedes Gewichtsmanagementprogramm die Bewegungssteigerung als Therapiekomponente beinhalten (DAG, 2006; NHLBI \& NAASO, 2000).

Bewegungstherapeutischen Konzeptionen und ihren Zielsetzungen wird in dieser sportwissenschaftlichen Arbeit großer Raum gewährt, wobei zunächst die geläufigsten deutschen und englischen Fachbegriffe für das weite Feld der Bewegungstherapie erklärt werden.

\subsection{Begriffserklärungen}

Im Rahmen dieser Arbeit wird analog zu den Definitionen des Deutschen Verbandes für Gesundheitssport und Sporttherapie (DVGS) Bewegungstherapie als Überbegriff und Sporttherapie als ein ihr untergeordneter Teilbereich verstanden (VandenAbeele \& Schüle, 2004).

Während im deutschsprachigen Raum die therapeutische Funktion und die pädagogisch-psychologischen Gesichtspunkte der Begriffe Bewegungstherapie und Sporttherapie hervorgehoben werden, sind die englischsprachigen Begriffe ohne psychotherapeutische Konnotationen zu verstehen.

In der englischsprachigen Literatur dominieren Termini wie Physical Activity, Exercise, Lifestyle Activity bzw. Lifestyle Physical Activity Interventions.

Physical Activity wird als „any bodily movement produced by skeletal muscles that results in energy expenditure" (Caspersen, Powell \& Christensen, 1985, zitiert nach Sallis \& Owen 1999, S. 10) verstanden.

Es handelt sich um einen globalen Begriff, der ausgeführte körperliche Aktivitäten, die in einer moderaten Intensität ausgeführt werden, mit einbezieht. Dazu zählen körperliche Aktivitäten (Alltagsaktivitäten) wie Gehen, Fahrradfahren, Rasen-mähen, Gartenarbeit, Tanzen, Blätterkehren, Schneeschauffeln etc..

Exercise als ein Aspekt der körperlichen Aktivität wird wie folgt definiert:

„Any planned, structured, and repetitive bodily movement done to improve or maintain one or more components of physical fitness - cardiorespiratory endurance (aerobic fitness), muscular strength, muscular endurance, flexibility and body composition" (Caspersen, Powell \& Christensen, 1985, zitiert nach Sallis \& Owen, 1999, p. 10). 
Einbezogen werden dynamische und andauernde Aktivitäten das Laufen, Schwimmen und Fahrradfahren mit der Zielsetzung einer Verbesserung oder Aufrechterhaltung der körperlichen Fitness oder der Gesundheit (U.S. Department of Health and Human Services, 1996; Sallis \& Owen, 1999).

Lifestyle Physical Activity wird definiert als „... the daily accumulation of at least 30 minutes of self-selected activities, which includes all leisure, occupational, or household activities that are at least moderate to vigorous in their intensity and could be planned or unplanned activities that are part of everyday life (Dunn, Andersen \& Jakicic, 1998).

Sie wird als eine alltägliche durch Muskelkraft bewirkte Bewegung definiert. Alltägliche Aktivitäten, die die Menschen durchführen können, sind beispielsweise: mit dem Hund gehen, Einkäufe tragen, Hausarbeit verrichten, mit den Kindern spielen, das Auto in einiger Entfernung vom Geschäft oder der Arbeitstelle parken etc. (U.S. Department of Health and Human Services, 2005; Catanese, Hyder, Poston \& Foreyt, 2003).

Die Problematik bezüglich der Begrifflichkeit im Bereich von Bewegung und Adipositasbehandlung liegt darin begründet, dass die Literatur vorwiegend aus dem angloame-rikanischen Sprachraum kommt. Die Definitionen von Sport und Training gehen mit denen des Deutschen nicht immer konform. Um Missverständnisse auszuschließen, zeigt die folgende Tabelle thematische Schlüsselbegriffe mit der für diese Arbeit relevanten Definition.

Tabelle 8: Definition von körperlicher Aktivität und verwandter Begriffe nach dem Europäischen Informationszentrum für Lebensmittel, (EUFIC; European Food Information Council) (2006)

Körperliche Aktivität Alle körperlichen Bewegungen, die einen Energieverbrauch zur Folge haben. Sie umfassen tägliche Routinetätigkeiten im Haushalt, beim Einkaufen und bei der Arbeit.

Bewegung Geplante und strukturierte, wiederholte Bewegungen um Fitness und Gesundheit zu verbessern. 
Sport

Körperliche Fitness
Körperliche Aktivitäten, die strukturierte Wettbewerbssituationen einschließen und durch Regeln bestimmt sind. In vielen europäischen Ländern wird der Begriff Sport benützt, um alle Bewegungs- und Freizeitaktivitäten einzuschließen.

Eine Reihe von Eigenschaften wie Ausdauer, Beweglichkeit und Kraft, die sich auf die Fähigkeit beziehen, körperliche Aktivität zu leisten.

\subsection{Bewegungsverhalten bei Adipositas}

Die meisten Experten und die neusten Richtlinien erkennen die Rolle der körperlichen Aktivität für die Ätiologie und die Behandlung der Adipositas an (Blair \& Leermakers, 2004; DAG, 2006; NHLBI \& NAASO, 2000; WHO, 2000).

Auch wenn es kein gesichertes Datenmaterial gibt, welches den genauen Anstieg der Prävalenz von Adipositas in der Welt aktuell belegt, gibt es Hinweise auf die ganz besondere Rolle der Bewegung in Zusammenhang mit der Entstehung und der Therapie von Adipositas (vgl. Kap. 2.3 und 2.5.1).

Adipositas entsteht unvermeidlich, sobald über einen längeren Zeitraum eine positive Energiebilanz besteht, welche die Ansammlung eines Übermaßes an Gewicht zur Folge hat.

Seit einiger Zeit sind die durchschnittliche Kalorienaufnahme und insbesondere der hohe Fettverzehr in Deutschland leicht rückläufig (Hauner \& Berg, 2000). Studien aus den USA zeigen ähnliche Ergebnisse mit einem stabilen oder leichten Anstieg der durchschnittlichen Gesamtenergieaufnahme (Blair \& Leermakers, 2004). Es wird kritisiert, dass keine repräsentativen Daten großer Stichproben vorliegen, die den Energieverbrauch oder die Veränderungen des Energieverbrauches in den letzen Jahren messen (Blair \& Leermakers, 2004; Bensimhon, Kraus \& Donahue, 2006) Vorhandene Daten über die Teilnahme an verschiedenen körperlichen Aktivitäten sind für die Bewertung des gesamten Energieverbrauchs unzureichend.

Aktuelle repräsentative Daten aus der amerikanischen Bevölkerung bezüglich der körperlichen Aktivitätslevel, zusammengefasst in einem Bericht „Physical Activity and Health: A report of the Surgeon General" (U.S. Departement of Health and Human Services, 1996), zeigen, dass es in den letzten Jahren minimale Änderungen 
des Prozentsatzes der Erwachsenen gab, die regelmäßig sportlich aktiv sind oder passiv sind (Blair \& Leermakers, 2004).

Ähnliche Ergebnisse sind in Mitteleuropa und Deutschland $\mathrm{zu}$ finden. Nach den Fragebogenangaben des Bundes Gesundheitssurveys von 1998 ist der Anteil der Inaktiven in der deutschen Bevölkerung, definiert als Menschen, die überhaupt keinen Sport in ihrer Freizeit betreiben, mit 43,8 \% der Männer und 49 \% der Frauen alarmierend hoch. $13 \%$ der Männer und 10,3 \% der Frauen sind wenigstens 2-4 Stunden pro Woche sportlich aktiv. Der Vergleich mit ähnlichen Daten des Nationalen Gesundheitsurveys von 1990 und 1992 zeigt, dass während dieser Jahre der Anteil der Männer, die sich mehr als zwei Stunden pro Woche sportlich betätigen, um 4,0 \% und bei den Frauen sogar um 5,9\% zugenommen hat. Allerdings ist auch der Anteil der Inaktiven unter den Männern um 2,3 \% gestiegen. Bei den Frauen hingegen ist der Anteil der Inaktiven um 3,5 \% gesunken (Mensink, 1999; Mensink, 2003).

\subsection{Bewegung als Ursache, Folge, Therapie und Prävention der Adipositas}

Nach der dargestellten aktuellen Datenlage könnte man die Schlussfolgerung ziehen, dass der totale Energieverbrauch eines Menschen in den letzten Jahren recht konstant geblieben ist (Blair \& Leermakers, 2004).

Dies wird als sehr unwahrscheinlich kritisiert, denn das Lebensumfeld wurde in Bezug auf die körperlichen Aktivitäten „toxisch“ (Brownell, 2002; Horgen \& Brownell, 2004). In der deutschsprachigen Literatur finden sich die ausdruckstarken Termini „obesogenic“ und „,adipogenen“, die auf eine die Adipositas begünstigende Umwelt verweisen (Huber, 2004; Rathmanner et al., 2006).

Deutlich wird die Bewegungsarmut durch die Verbreitung sitzender Tätigkeiten, wie des Surfens im Internet oder des Spielens von Computerspielen. Zu diesen sitzenden Freizeitaktivitäten hinzu kommt die allgegenwärtige Benutzung elektrischer Geräte zur Ausführung von Tätigkeiten, die früher durch menschlichen Energieverbrauch erfolgten. Elektrische Türen, Lifte statt Treppen, Rolltreppen, automatische Fensterheber und andere muskuläre Arbeit sparende Geräte stehen im Überfluss für den Haus- oder Arbeitsgebrauch zur Verfügung. Sie sind Beispiele einer modernisierten, jedoch bewegungsarmen Gesellschaft (Blair \& Leermakers, 2004). 
In einer derartigen Umwelt muss der Mensch bewusst nach Gelegenheiten für körperliche Aktivitäten in seinem Leben suchen (Mannix, Dempsey, Engel, Schneider \& Busk, 1999).

Nach Blair \& Leermakers (2004) und Wirth (2003) änderte sich der menschliche Energieverbrauch, allerdings gäbe es für diese Annahme keine verfügbaren Daten, um dieses zu belegen. Blair \& Leermakers (2004) kritisieren weiter, dass mehr Forschungsgelder für die Entwicklung von Instrumenten, die Energiezufuhr messen, als für die Entwicklung und Validierung von Instrumenten für den Energieverbrauch investiert würden.

Obwohl keine randomisierten, kontrollierten Studien an großen Stichproben vorliegen, die die körperliche Inaktivität als eine Ursache für die Adipositas an großen Bevölkerungszahlen auswerten, gibt es jedoch eine Reihe von Beobachtungsstudien, die einen kausalen Zusammenhang unterstützen. Nach einer Review von 8 prospektiven Studien zusammengetragen in einem Roundtable Consensus Statement der American College of Sports Medicine (ACSM), berichten Forscher über eine gemäßigt starke Beziehung zwischen einem niedrigen körperlichen Aktivitätslevel und dem Risiko der Entwicklung von Adipositas (Grundy et al., 1999, zitiert nach Bensimhon et al., 2006).

Die aktuellsten Daten aus dem deutschen Raum, erfasst durch den telefonischen Gesundheitssurvey 2003, zeigen, dass bei Männern und Frauen die Anteile der Adipösen in der Gruppe der sportlich Aktiven (Sporttreiben $>2$ Stunden/Woche) deutlich unter denen in der Gruppe der weniger Aktiven (Sport treiben $<2$ Stunden/ Woche) liegen (Mensink, Lampert \& Bergmann, 2005).

Folgt man der allgemeinen These, dass übermäßiges inaktives Verhalten ein Risikofaktor für eine Gewichtszunahme bei Erwachsenen darstellt, und die technologische Revolution der letzten Jahre des 20. Jahrhunderts eine wichtige Ursache der Adipositasepidemie in den wohlhabenden Nationen ist, dann ergeben sich folgende ergänzende Fragen:

- Welchen Rang nimmt die körperliche Inaktivität in der Hierarchie der Risikofaktoren ein?

- Sind alle Menschen gleich gefährdet an Gewicht zuzunehmen, wenn sie inaktiv sind? 
- Gibt es besondere Merkmale, die einige Gruppen zu Inaktivität und zu einer damit assoziierten Gewichtszunahme prädisponieren (z.B. niedriger sozialer Status und Bildungsniveau, Alter, Geschlecht und Wohnort)?

- Sind es Gene, die die Menschen zur körperlichen Inaktivität oder zu intensiven Aktivitäten prädisponieren?

- Gibt es einen Grenzwert an körperlicher Aktivität, der vor einer Gewichtszunahme schützt?

- Wenn es so ist, ist dieser für alle Menschen und gegen alle diätetischen Ansätze gleich?

- Ist es in Bezug auf die Gewichtszunahme nötig, zwischen den verschiedenen Intensitäten der körperlichen Aktivitäten zu unterscheiden, oder handelt es sich um eine einfache Kumulation der Energieausgabe?

- Welchen Einfluss hat körperliche Aktivität auf den Zusammenhang von Adipositas und den assoziierten Komorbiditäten?

(Prentice \& Jebb, 2000, S. 247).

\subsection{Probleme bei der Erfassung von körperlicher Aktivität bei Adipositas}

Eine präzise Bemessung der körperlichen Aktivitäten unter Feldbedingungen ist für eine genaue Beschreibung der Epidemiologie von Bewegungsarmut bei Adipositas und für die Entwicklung entsprechender Interventionen zur Förderung einer Lebensstiländerung entscheidend. Es ist notwendig, die körperliche Aktivität zu erfassen, um Ätiologie, Prävention und Therapie der Adipositas zu verstehen (Sallis \& Zabinski, 2002). Körperliche Aktivität ist ein komplexes und multidimensionales Verhalten, dessen genaue Erfassung eine Herausforderung für Fachleute, Forscher und Therapeuten bleibt, insbesondere bei Bevölkerungen unter Feldbedingungen (LaMonte, Ainsworth \& Tudor-Locke, 2003).

Bei der Erforschung körperlicher Aktivität in Bezug auf die Adipositas gibt es widersprüchliche Ergebnisse, die epidemiologische wie experimentelle Einschränkungen aufweisen (Grillo et al., 1993; Prentice \& Jebb, 2000).

Aus diesem Grund sollen kurz die Schwierigkeiten bei der Einschätzung der Beziehung zwischen körperlicher Aktivität und der Adipositas erläutert werden. 


\subsubsection{Kritik an den bestehenden Messverfahren}

Es gibt keine Technik, die als ein goldener Standard für die Erfassung der körperlichen Aktivität oder des Energieverbrauchs für Übergewichtige unter Feldbedingungen gilt. Es gibt Bemühungen zur Entwicklung von besseren Methoden einer quantitativen Bemessung des Gesamtenergieverbrauchs und der körperlichen Verhaltensmuster.

Allgemein ist zu kritisieren, dass sich die derzeit angewandten Verfahren wenig von den Verfahren für Normalgewichtige unterscheiden. Je nach Ausmaß des Übergewichts messen diese Techniken weniger genau als erwartet (LaMonte et al., 2003; Schutz, Weinsier \& Hunter, 2001).

a) Das Metabolische Äquivalent

Prädiktive Kalorienverbrauchsberechnungen, Gleichungssysteme wie das Metabolische Äquivalent (gemessen in MET) wurden für die Einschätzung des Energieverbrauchs aus direkten und indirekten Messungen der körperlichen Aktivität an aktiven weißen Männern und Frauen mit einem durchschnittlichen Body Mass Index und Körperfettanteil entwickelt. Aus dem Grund sind sie für Adipöse inadäquat und neigen dazu, den durch Bewegung tatsächlich entstandenen Energieumsatz übergewichtiger Personen zu unterschätzen. Rasse und Volkszugehörigkeit sind ein wichtiger modifizierender Faktor für den Energieverbrauch durch die Bewegung und den Grad des Körperfettes und sollten bei der Erfassung und Verordnung körperlicher Aktivität und Gewichtsabnahme berücksichtigt werden. Metabolische Äquivalente (METs) geben absolute Skalen für den zugeteilten Intensitätsgrad der körperlichen Aktivitäten. Grundlage sind aber immer noch subjektiv geführte Bewegungs-tagebücher und Fragebogenitems, so dass falsche Schlussfolgerungen für den habituellen Grad des Energieverbrauchs bei den Menschen mit Adipositas gezogen werden können. Der schlechte konditionelle Zustand von Adipösen kann hier eine verzerrende Rolle spielen (Schutz et al., 2001; LaMonte et al., 2003).

b) Technische Geräte zur Bewegungsmessung

Bewegungssensoren wie Pedometer (Schrittmesser) und Accelerometer (Beschleunigungsmesser) sind wenig präzise in der Erfassung langsamer Geschwindigkeiten. Ebenso können sie Abstufungen in der Belastungsintensität nicht erfassen. 
Daher gelten sie als ungenau (LaMonte et al., 2003). Die Geräte sind häufig nicht wasserdicht und unterschätzen Aktivitäten, die nicht durch kräftige vertikale Bewegungen des Rumpfes gekennzeichnet sind, wie Hausarbeit, Radfahren, Gehen mit Gewichten (Sallis \& Zabinski, 2002).

c) Fragebögen

Verfahren, die in großen epidemiologischen Studien gut einsetzbar sind, sind Fragebögen, die Verhaltensmuster der körperlichen Aktivität, Inaktivität und damit den in Beziehung stehenden Energieverbrauch messen. Sie sind jedoch häufig weder präzis noch reliabel. Messungen, die auf Selbstberichten durch kurze häufig einfaktorielle Fragebögen beruhen, haben eine niedrige Validität. Solche Messungen sind sehr anfällig, da die Aktivitäten, insbesondere von übergewichtigen und adipösen Menschen, überschätzt werden (Sallis \& Zabinski, 2002; Prentice, 2002; Lichtman et al., 1992). Dieses Phänomen wird im angloamerikanischen Sprachraum als „overreporting“ bezeichnet.

d) Doppel-Isotopen-Methode

Die Doppel-Isotopen-Methode (double labeled water) gewährleitstet eine durchgängige Messung des Gesamtenergieverbrauchs und ist sehr robust bei der Beobachtung von Effekten. Dadurch wird der genaue Energieverbrauch durch die körperliche Aktivität berechnet. Diese Technik ist jedoch komplex, laborintensiv, teuer und aus dem Grund für Studien mit großen Stichproben nicht durchführbar (Prentice, 2002).

So wird zwischen zwei Extremen, der breiten Einsetzbarkeit bei geringem Aufschlussvermögen und der geringen Einsetzbarkeit bei hohem Aufschlussvermögen, eine Kombination von verschiedenen Methoden empfohlen. Die kombinierte Anwendung verschiedener Verfahren ohne Korrelationsfehler (validiert gegen die Doppel-Isotopen-Methode) und deren Synthese in einem zusammengefassten Aktivitätsindex oder einer multifaktoriellen Analyse ist theoretisch der geeignetste Weg für eine genaue Bemessung der habituellen körperlichen Aktivität (LaMonte et al., 2003; Prentice \& Jebb, 2000). 


\subsubsection{Methodische Probleme bei der Erfassung von körperlicher Aktivität}

a) Körperliche Aktivität ist erst in jüngerer Zeit als eine Variable für die Gesundheitsforschung von Interesse. Forschung an habituellen körperlichen Aktivitätsmustern als ein bestimmender Faktor für die körperliche Fitness und Adipositas hat somit eine kurze Geschichte. Es besteht ein Mangel an Baseline Daten, welcher zu unbefriedigender Ersatzmessung der Inaktivität anhand von Zahlen zu PKW Zulassungen oder der Nutzung des Fernsehers geführt hat (Prentice \& Jebb, 2000).

b) Ein weiteres Problem ist die Schwierigkeit, den passenden Zeitpunkt zur Erfassung der körperlichen Aktivitäten und ihrer Folgen für die Adipositas zu finden. Bewegung zeigt tief greifende gesellschaftliche und individuelle Abweichungen, die Folgevariable Adipositas stellt die kumulativen Effekte von Jahren oder Jahrzehnten eines Ungleichgewichts der Energiebilanz dar. Insbesondere prospektive Studien fanden unterschiedliche Verbindungen zwischen Aktivität und Gewichtszunahme. Sie waren abhängig davon, ob die Aktivität während der Baseline oder der Follow-up Periode oder in einer Kombination von beiden gemessen wurde (Prentice \& Jebb, 2000).

c) Für die Interpretation der Forschungsergebnisse kommt erschwerend hinzu, dass körperliche Aktivität eine sehr komplexe Synthese aus kleinen körperlichen Bewegungen („herumzappeln“), Alltagsbewegungen und motorische Aktivität, die mit Arbeit oder Sport assoziiert wird, ist. Die Forschung befindet sich in einem frühen Stadium des Verstehens der Bedeutung der verschiedenen Komponenten für die Aufrechterhaltung der den Metabolismus betreffenden Gesundheit. Es wird vorgeschlagen, dass Inaktivität nicht einfach als das Gegenteil von Aktivität gesehen werden sollte. Inaktivität (Fernsehen, Computerbenutzung etc.) hat Einfluss auf den Metabolismus und den Gefäßtonus. Sitzende Verhaltensmuster können darüber hinaus mit anderen negativen Verhaltensmustern wie exzessivem ständigem Essen und Alkoholkonsum einhergehen. Der Erfolg von Gewichtsverlustprogrammen für Erwachsene, die stärker auf die Aufhebung der Inaktivität als auf die Förderung eines Trainings zielen, untersteichen die Bedeutung dieser Dimension für das Problem (Prentice \& Jebb, 2000; Saris, 2002). 
Unter Berücksichtigung all dieser methodologischen Schwierigkeiten kommt man zu der Schlussfolgerung, dass die Heterogenität der Forschungsergebnisse das „. . .result from the complex nature of the mechanism underlying weight change and from the limited techniques available for assessing diet and physical activity among the general population" ist (Williamnson, 1966, zitiert nach Prentice \& Jebb, 2000, S. 250).

Trotz aller Widersprüche und der aussageschwachen Qualität der zur Verfügung stehenden Daten gibt die Tatsache, dass körperliche Aktivität stark in Beziehung zu einer erfolgreichen langfristigen Gewichtsaufrechterhaltung steht, einen deutlichen Hinweis auf die Bedeutung der körperlichen Aktivität im Zusammenhang mit Adipositas. Die Tatsache, dass eine derart starke Verbindung aus groben Messungen entstehen konnte, macht diese Schlussfolgerung noch robuster (Prentice \& Jebb, 2000).

Im Weiteren sollen die Auswirkungen der körperlichen Aktivität und des Trainings im Rahmen der Adipositastherapie dargestellt werden. Viele der Ergebnisse und Befunde kommen aus dem amerikanischen Raum. Aus diesem Grund werden die Begriffe Physical Activity oder Exercise verwendet (vgl. Kap. 4.1).

\subsection{Bewegungstherapie und Gewichtskontrolle}

Die Hauptintervention für einen Gewichtsverlust beruht nach wie vor auf der Reduktion der Energiezufuhr und der Steigerung der Energieausgabe. Sportliche und körperliche Aktivitäten sind mit einer Gewichtsabnahme assoziiert. Dieser Zusammenhang ist extensiv mit verschiedenen Studiendesigns untersucht worden. Metaanalysen und Übersichtsarbeiten versuchten die vorhandenen Mengen von Daten und Ergebnissen zusammenfassend zu analysieren.

Zunächst scheint körperliche Aktivität einer Zunahme des initialen Gewichts vorzubeugen. Eine Beziehung zwischen körperlicher Aktivität und Gewichtsverlauf konnte in Längsschnittuntersuchungen von Williamson et al., (1993), French et al., (1994) und DiPietro, Kohl, Barlow \& Blair (1998) gezeigt werden. Die Daten weisen auf die präventive Funktion eines gesunden und aktiven Lebensstils bzgl. einer Gewichtszunahme hin (Blair \& Leermakers, 2004; Jakicic, 2003). 
Körperliche Aktivität als einzelne Intervention erzielt einen geringen Gewichtsverlust. Es bedarf extrem hoher Umfänge an körperlichen Aktivitäten, um eine für eine Gewichtsabnahme notwendige Energieausgabe zu erreichen. Eine allein durch Bewegung induzierte Gewichtsabnahme ist für übergewichtige und vor allem für adipöse Personen eine ungeheuere Herausforderung. Viele der übergewichtigen oder adipösen Personen sind aufgrund ihrer Passivität körperlich nicht leistungsfähig. Sie würden die zur Gewichtsabnahme erforderlichen Umfänge und Intensitäten der sportlichen und körperlichen Aktivitäten nicht ausführen können. Kurz gesagt: „Übergewichtige Personen haben nicht das Leistungsvermögen, solche großen Energiemengen auszugeben“ (Blair \& Leermakers, 2004).

Körperliche Aktivität allein ist kein probates Mittel zur Gewichtsreduktion von 510\% (übliches Therapieziel) des Körpergewichts (Wirth, 2003). Zu diesem Schluss kommen viele empirische Untersuchungen, u. a. die Metaanalyse von Miller, Koceja und Hamilton (1997), in der 493 Studien auf ihre Effizienz bzgl. des Gewichtsverlusts hin analysiert wurden. Die Autoren verglichen sporttherapeutische und ernährungstherapeutische Interventionen sowie die Kombination der beiden Interventionen. Es konnte gezeigt werden, dass ein 21-wöchiges Aerobicübungsprogramm nur 2,9 kg Gewichtsverlust erzeugen konnte. Im Vergleich hierzu ließ sich durch ein 15-wöchiges Reduktionskostprogramm eine Gewichtsreduktion von $11 \mathrm{~kg}$ erreichen.

Sporttherapeutische Maßnahmen sind bei kurzfristiger Therapie der Ernährungstherapie deutlich unterlegen, ein Jahr nach Therapieende sind die Erfolge jedoch ähnlich.

Eine Aussage für eine dauerhafte Gewichtsstabilisierung lässt sich nach Meinung der Autoren nicht treffen, da entsprechende Untersuchungen fehlen.

Eine Kombination aus Reduktionskost und gesteigerter Bewegung ist allerdings beiden Konzepten als Monotherapie eindeutig überlegen (Miller, Koceja \& Hamilton, 1997; NHLBI, 1998; Curioni \& Lourenco, 2005; Jakicic \& Otto, 2005).

$\mathrm{Zu}$ ähnlichen Ergebnissen kommen auch andere Metaanalysen wie die von Garrow und Summerbell (1995) oder Vortuba, Horwitz und Schoeller (2000). Insbesondere in der Übersichtsarbeit von Vortuba et al. (2000) untersuchen die Autoren neben der Frage nach der Bedeutung körperlicher Aktivität in Bezug auf die Kombination verschiedener Therapieelemente (Diät und/oder Sporttherapie) die Rolle der Trainingsintensität und der Trainingsart (Ausdauer- versus Krafttraining) und deren 
Effekte auf Gewichtsverlust bzw. den Erhalt des reduzierten Gewichts. Zusammenfassend konnte hier gezeigt werden, dass die Kombination einer Aktivitätssteigerung mit einer Kalorienrestriktion im Vergleich mit diätetischen Maßnahmen an sich geringe zusätzliche Ergebnisse an einer Gewichtsreduktion hat (Vortuba, Horwitz \& Schoeller, 2000).

Obwohl körperliche Aktivität nicht die effizientere Methode für eine Gewichtsreduktion ist, lassen die Ergebnisse vieler empirischer Untersuchungen darauf schließen, dass die Hauptbedeutung vermehrter körperlicher Aktivität bei der Prävention und bei der Gewichtserhaltung (,weight maintenance „) liegt (Vortuba, Horwitz \& Schoeller, 2000; Wirth, 2003; Blair \& Leermakers, 2004).

Das Hauptproblem jeder Adipositastherapie nach einer Gewichtsreduktion ist die langfristige Stabilisierung des neuen Körpergewichts. Die Rezidivraten sind auch bei multidisziplinär angelegten Programmen außerordentlich hoch (Hauner \& Berg, 2000).

Es wird berichtet, dass übergewichtige Personen, die nach einer erfolgreichen Gewichtsreduktion ihr Gewicht wiedererlangten („relapsers“), nicht regelmäßig körperlich aktiv waren, während übergewichtige Personen, die ihre Gewichtsreduktion erhalten konnten (,mainteners“), regelmäßig körperlich aktiv waren (Blair \& Leermakers, 2004).

Die o.g. Metaanalysen wie eine Reihe anderer epidemiologischer Studien unterstützen diese These, die auch in der viel zitierten Studie von Pavlou, Krey \& Steffee (1989) bestens demonstriert wird.

Der Hauptteil der Daten, die die These einer Schlüsselrolle der Bewegung für die Gewichtserhaltung unterstützen, kommt von Beobachtungsstudien und post hoc Analysen der Gewichtsreduktionsuntersuchungen. Überzeugende Untersuchungen kommen aus dem Amerikanischen (National Control Registry). Seit 1994 sind in dieser Studie Daten von über 3000 Personen, die ihre Gewichtsreduktion von $\geq 13,62$ $\mathrm{kg}$ über mehr als 1 Jahr gehalten haben erfasst. Es zeigte sich, dass diese Gewichtsstabiltät durch 3 Verhaltensstrategien erreicht wurde:

- diätetische Maßnahmen (fettarme, kohlenhydrat- und ballaststoffreiche Ernährung),

- regelmäßiges self- monitoring des Körpergewichts und der Ernährung und 
- regelmäßige körperliche Aktivität (91\% der Teilnehmer berichteten über regelmäßige körperliche Aktivitäten, wie eine Stunde schnelles Walking am Tag).

Die Studienteilnehmer sahen in der Bewegung ihre „Hauptwaffe“ im Kampf um die Aufrechterhaltung des Gewichtsverlusts (Wing \& Hill, 2001).

Eine systematische Übersichtsarbeit von 46 Beobachtungsuntersuchungen und randomisierten kontrollierten Studien über die Rolle der Bewegung bei der Gewichtserhaltung zeige jedoch einen inkonsistenten Zusammenhang zwischen körperlicher Aktivität und Gewichtserhaltung. Bei den kontrollierten Studien, die in diese Analyse eingeschlossen waren, konnte jedoch ein allgemeiner Trend einer besseren Gewichtserhaltung in den sportlichen Gruppen im Vergleich zu den Kontrollgruppen gezeigt werden. Die Intensität der Aktivität verhielt sich analog zum Ausmaß des gehaltenen Gewichtes (Fogelholm \& Kukkonen-Harjula, 2000).

Wing (1999) beschreibt sechs Studien mit einem Follow -up von einem Jahr und länger, die Ernährungstherapie allein mit Ernährungstherapie in Kombination mit Sporttherapie verglichen. Obwohl alle 6 Untersuchungen mit der Kombination von Ernährung und Bewegung größere langfristige Gewichtsabnahmen zeigten, deuten nur zwei von sechs einen statistisch signifikanten Unterschied an (Wing, 1999). Das Misslingen, die Vorteile derartiger Behandlungskombinationen wissenschaftlich zu belegen, hängt primär mit der Schwierigkeit zusammen, Teilnehmer zu gewinnen, die für eine längere Zeit ihr körperliches Training beibehalten. Es macht deutlich, wie wichtig es für die Adipositastherapie ist, nach der Einführung der sportlichen Aktivitäten diese auch zu erhalten (,exercise adherence“) (Wing, 2004).

Das Beibehalten des reduzierten Körpergewichts ist nicht der einzige positive Effekt einer andauernden Änderung des Bewegungsverhaltens. Adipöse Personen sollten darüber informiert sein, dass unabhängig von einer Gewichtsreduktion Bewegung Begleiterscheinungen der Adipositas (z.B. hohem Blutdruck) entgegenwirkt. Und auch wenn keine Gewichtsabnahme erfolgt, können positive Veränderungen bzgl. der Körperzusammensetzung stattfinden (Mannix et al., 1999).

\subsection{Wirkungsmechanismen der körperlichen Aktivität bei der Gewichts- kontrolle}

Nach den o.g. Forschungsergebnissen wird die These unterstützt, dass das körperliche Training einen Prädiktor für die Gewichtsbeibehaltung darstellt. Studien 
zeigen, dass Personen, die trainieren, ihre Gewichtsverluste eher beibehalten als solche, die nicht trainieren. Weniger klar ist, warum diese Beziehung existiert und welche Mechanismen hier zugrunde liegen.

Das Verständnis der möglichen Wirkungsmechanismen körperlicher Aktivität beim Gewichtsmanagement ist zwingend erforderlich, um die körpertherapeutischen Interventionen optimal zu gestalten.

Es werden vorwiegend zwei Mechanismen dargestellt, die dann auch dementsprechend zwei unterschiedliche Verständnisse und Handlungsweisen der sportlichen Trainings erfordern. Wenn der Hauptmechanismus der Kalorieverbrauch sein sollte, dann soll das Ziel des Bewegungsprogramms die Maximierung der Energieausgabe sein. Auf der anderen Seite, wenn das körperlichre Training die Gewichtskontrolle durch psychologische Mechanismen beeinflusst, soll das Bewegungsprogramm so gestaltet werden, dass es zu den gewünschten psychologischen Ergebnissen führen kann. Die praktische Umsetzung bei den zwei Bewegungsprogrammen kann dadurch äußerst unterschiedlich sein.

Baker und Brownell (2000) fassen mögliche Wirkungsmechanismen der körperlichen Aktivität für die Gewichtskontrolle nach ihren Evidenzgrad und mögliche Pfadmodelle zusammen. Sie betonen die potentielle Bedeutung (der selten diskutierten Mechanismen) der psychologischen Effekte des sporttherapeutischen Trainings (Baker \& Brownell, 2000).

Tabelle 9: Zusammenfassung der Wirkungsmechanismen und ihre wissenschaftliche Evidenz (nach Baker und Brownell, 2000, 315)

\section{Wirkungsmechanismus}

\section{Wissenschaftliche Evidenz}

Energieausgabe

Verminderter Appetit

Verminderte Nahrungsaufnahme /erhöhte Diätaufrechterhaltung* +

Änderung der Nahrungszusammensetzung** $\quad-+$

Erhaltung der fettfreien Körpermasse

Senkung des Grundumsatzes

$-=$ kein klarer Beleg für diesen Mechanismus; $+=$ teilweise belegter Mechanismus;

$++=$ wissenschaftlich belegter Mechanismus; $-+=$ widersprüchliche Belege;

* Stichproben Übergewichtiger; $* *-=$ kurzanhaltend; $* *+=$ dauerhaft 


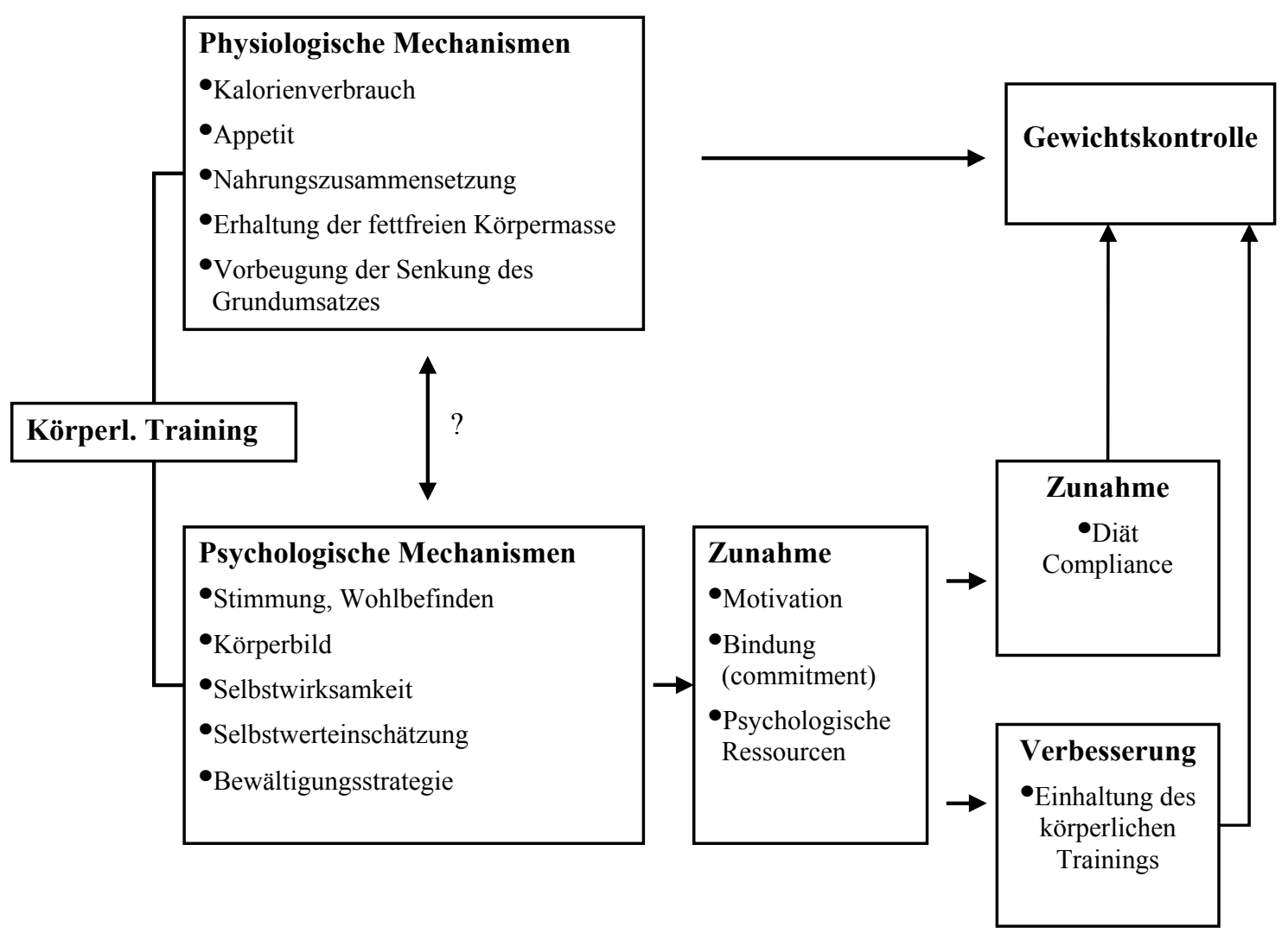

Abbildung 6: Vorgeschlagene Mechanismen und mögliche Pfadmodelle der Verbindung körperliches Training und Gewichtskontrolle (nach Baker und Brownell, 2000, 315)

\subsubsection{Energieausgabe}

Körperliche Aktivität kann die Energiebilanz dadurch beeinflussen, dass die Kalorienausgabe erhöht wird. Es ist jedoch unwahrscheinlich, dass dieses ein für die angestrebte Gewichtskontrolle ausreichender Mechanismus ist. Menschen mit Adipositas sind häufig nicht in der Lage einen signifikanten Kalorienverbrauch zu erreichen, insbesondere $\mathrm{zu}$ Beginn eines Bewegungsprogramms. Ihre Leistungsfähigkeit wie auch das Übermaß an Gewicht beschränken die Dauer und die Intensität der körperlichen Aktivität (Blair \& Leermakers, 2004).

Die Gewichtsverluste bei Menschen, die trainieren, liegen in der Regel unter den Erwartungen. Auch subjektiv sehr anstrengend wahrgenommene körperliche Aktivitäten ergeben eine relativ niedrige Energieausgabe. Körperliche Aktivitäten erzielen kleine Änderungen in der Energiebilanz, welche sich über die Zeit möglicherweise 
summieren können, Energieausgabe ist jedoch wahrscheinlich eine untergeordnete Komponente im Gesamtzusammenhang von körperlicher Aktivität und Gewichtskontrolle (Baker \& Brownell, 2000).

\subsubsection{Appetit, Nahrungsaufnahme und Nahrungszusammensetzung}

Ein weiterer wissenschaftlich untersuchter Mechanismus ist die Appetit mindernde Wirkung der körperlichen Aktivität. Appetit und Energieaufnahme sind verschiedene Variablen, die nicht unbedingt hoch miteinander korrelieren. Die Studienergebnisse bzgl. der beiden Variablen sind nicht einheitlich (Mannix et al., 1999). Sie konzentrierten sich vielmehr auf die Frage, ob körperliches Training zu einem Energieausgleich führt. Nur wenige untersuchten, ob das körperliche Training zu einer besseren Compliance der diätetischen Maßnahmen beitragen konnte. Ähnlich inkonsistent sind die Ergebnisse aus der Literatur für die Rolle der körperlichen Aktivität bei der Nahrungszusammensetzung. Es stellt sich die Frage, ob körperliche Aktivität zu Veränderungen des Verhältnisses Kohlenhydrate versus Fett während der diätetischen Maßnahmen führt.

Baker und Brownell (2000) fassen zusammen, dass körperliche Aktivitäten weder eine erhöhte Energieaufnahme noch einen herabgesetzten Appetit oder signifikante Veränderungen bei der Auswahl der Nahrungszusammensetzung zur Folge haben (Baker \& Brownell, 2000).

Ergänzend muss angemerkt werden, dass die Effekte des körperlichen Trainings auf die Energieaufnahme und den Appetit eine komplexe Angelegenheit sind, die von mehreren Faktoren, u. a. der Intensität und Dauer des Trainings und dem Essensanreiz, beeinflusst wird (Mannix et al., 1999). Personen, die sportlich aktiv sind, und die durch körperliche Aktivität vermehrte Energieausgabe nicht ausgleichen, erzeugen eine negative Energiebilanz. Des Weiteren gibt es provokative Befunde, die zeigen, dass körperliche Aktivität mit einer verminderten Energieaufnahme oder zumindest mit einer besseren Einhaltung der verordneten Reduktionskost verknüpft sein kann (Baker \& Brownell, 2000).

\subsubsection{Körperzusammensetzung}

Wie viele Studien und Metaanalysen zeigen, besteht ein Konsens über den Einfluss der körperlichen Aktivität auf die Körperzusammensetzung. Körperliche Aktivität regt die Fettreduktion an. Gleichzeitig führt sie zu einem Erhalt oder einer Zunahme 
der Muskelmasse, welche die absolute Menge der Gewichtsreduktion beeinflussen kann. Wenn das Gewicht nur durch diätetische Maßnahmen verloren wird, können bis zu $30 \%$ der Gewichtsabnahme aufgrund einer Reduktion der fettfreien Muskelmasse entstehen (Baker \& Brownell, 2000; Vortuba, Horwitz \& Schoeller, 2000; Bensimhon et al., 2006).

Mehrere große Beobachtungsuntersuchungen mit tausenden von Teilnehmern zeigen, dass körperliche Aktivität einen positiven Effekt auf die Körperfettverteilung hat, gemessen am Bauchumfang, dem Verhältnis von Taillen- zu Hüftumfang und Taillen- zu Oberschenkelumfang.

Daten aus kontrollierten Studien zu dem gleichen Thema stellen diese Ergebnisse teilweise in Frage (NHLBI, 1998).

Ergebnisse aus Kurzzeitstudien ( $<16$ Wochen), die einen relativ hohen Energieverbrauch erzielen (ca. $9320 \mathrm{kcal} /$ Woche), zeigen eine Fettreduktion im Verhältnis zum Ausmaß der ausgeführten körperlichen Aktivität.

Im Gegensatz dazu zeigen kontrollierte länger andauernde Studien ( $>26$ Wochen) mit einem wesentlich niedrigeren Energieverbrauch (ca. $4620 \mathrm{kcal} /$ Woche) keine deutliche Korrelation zwischen körperlicher Aktivität und Fettverlust.

In der kontrollierten Studie von Wood et al., (1988) wurden 131 Männer mit Adipositas randomisiert in 3 Gruppen eingeteilt (nur Diätgruppe, Trainingsgruppe, passive Kontrollgruppe). Nach einem Jahr verlor die Diätgruppe im Durchschnitt 7,2 $\mathrm{kg}$ und die Trainingsgruppe 4,0 kg. Die Diätgruppe verlor 1,3 kg Muskelmasse, während die Trainingsgruppe $0,1 \mathrm{~kg}$ zunahm. Dieses machte die aktuelle Differenz bzgl. der Fettreduktion in den Gruppen kleiner (5,9 vs. 4,1 kg) (Wood et al., 1988).

Diese Ergebnisse sind von großer Bedeutung, da das therapeutische Ziel der Adipositastherapie weniger auf die absolute Reduktion des Körpergewichts gerichtet ist, sondern vielmehr auf die Verminderung der Fettmasse, speziell des intraabdominalen Fettes, unter weitgehendem Erhalt der Muskelmasse ausgelegt ist (Vögele, 2003; Wirth, 2003).

\subsubsection{Grundumsatz}

Eng verbunden mit den Änderungen der Körperzusammensetzung ist auch der Einfluss der körperlichen Aktivität auf den Grundumsatz. Eine Restriktion der Energieaufnahme führt $\mathrm{zu}$ einer substantiellen Senkung der Grundumsatzrate (Resting Metabolic Rate), welches das Ergebnis des Verlusts der fettfreien 
Körpermasse ist (Baker \& Brownell, 2000). Die wichtigste Determinante des Grundumsatzes als eine Komponente des Energieverbrauchs ist die fettfreie Körpermasse (Hauner \& Berg, 2000). Körperliche Aktivität, die mit einer Reduktionskost kombiniert wird, führt zu einem größeren Fettverlust und einer Erhaltung der fettfreien Körpermasse, welche wiederum der Senkung der Grundumsatzrate vorbeugen oder diese reduzieren kann (Wirth, 2000).

Allerdings bleiben die Befunde bzgl. der vorbeugenden Rolle der körperlichen Aktivität bei der Senkung der Grundumsatzrate, die durch eine Diät induziert ist, widersprüchlich. Während in der Metaanalyse von Ballor und Poehleman (1994) keine signifikanten Effekte von Training (Aerobic) auf den Grundumsatz während eines Gewichtsverlusts gefunden wurden, zeigt die Metaanalyse von Thompson, Manore \& Thomas, (1996), dass die Aufnahme körperlicher Aktivitäten in Verbindung mit einer hypokalorischen Kost der Senkung des Grundumsatzes nicht vorbeugen, sie jedoch minimieren konnte (Baker \& Brownell, 2000).

Die Rolle des Trainings in der Gewichtsstabilisierungsphase ist in ihren Auswirkungen auf den Grundumsatz entscheidend. Durch körperliche Aktivität bleibt fettfreie Körpermasse erhalten und der Rückgang der Grundumsatzrate wird vermindert. Dieses hat einen positiven Effekt auf die Gewichtsstabilisierung (Vögele, 2003).

\subsubsection{Psychologische Mechanismen}

Eine Reihe psychologischer Faktoren sind eng mit dem körperlichen Training und der Gewichtskontrolle bzw. Adipositas verbunden. Zunächst werden körperliche Aktivität und Training von psychologischen Faktoren beeinflusst.

Die körperliche Aktivität führt nach aktuellen Untersuchungsergebnissen zu einer Verbesserung des Wohlbefindens und steigert das Selbstwertgefühl.

Diese positiven Effekte übertragen sich auf andere Lebensgebiete und können so auch zu einer verbesserten Diäteinhaltung führen (Baker \& Brownell, 2000).

Der aktuelle Stand der sportwissenschaftlichen und sportpsychologischen Literatur weist auf verschiedene mögliche Effekte des körperlichen Trainings auf die psychologischen Faktoren, welche in Zusammenhang mit der Gewichtskontrolle stehen, hin. Diese Faktoren sind Stimmung (mood), Körperimage (body image), Selbstwertgefühl (self-esteem), Selbstwirksamkeit (self efficacy) und Bewältigungsstrategien (coping) (Baker \& Brownell, 2000). 
Es besteht konsistente Evidenz, dass das körperliche Training mit einer Erhöhung des Wohlbefindens und der Stimmung einhergeht. Positive Veränderungen des allgemeinen Wohlbefindens und der speziellen Faktoren wie Depression und Angst können zu einem verbesserten psychologischen „Klima“ führen, in dem es Personen vermehrt gelingt, kognitive, emotionale Ressourcen und die Motivation und Energie für ein langfristiges Engagement zur Gewichtsreduktion aufrechtzuerhalten.

Negative Gefühle sind häufig Auslöser für das Essen und für Essanfälle. Verbesserungen des allgemeinen Wohlbefindens könnten die Frequenz dieser Gemütszustande reduzieren. Dieses könnte eine wichtige Auswirkung auf die Aufrechterhaltung der Gewichtsreduktion haben.

Es gibt ausreichend Hinweise durch verschiedene Metaanalysen, die die antidepressive und anxiolytische Wirkung des körperlichen Trainings bei klinischen und nicht klinischen Stichproben gut belegen. Trotz der methodologischen Kritik an vielen Studien, die eine vorsichtige Interpretation der Ergebnisse zwingend macht, gilt als belegt, dass körperliches Training eine direkte verbessernde Wirkung auf die Stimmung und das Wohlbefinden hat. Bewegung reduziert Stress, Depression und Angst (Mannix et al., 1999; Biddle \& Mutrie, 2001; Fuchs, 2003).

Diese direkte Wirkung, insbesondere auf die Stimmungslage, ist bei beiden Geschlechtern und bei allen Altersgruppen zu finden. Sie ist nahezu unabhängig von der Art, Dauer und Intensität des Trainings. Körperliches Training wird als eine nützliche kurzfristige Methode zur Selbstregulation der Stimmung favorisiert (Yeung, 1996, zitiert nach Baker \& Brownell, 2000).

Es stellt sich die Frage, ob ähnliche Ergebnisse bei Menschen mit Adipositas zu finden sind. Eine Studie von Foreyt, Brunner, Goodrick, St Jeor \& Miller (1995) an übergewichtigen Erwachsenen untersuchte psychologische Variablen, die über einen Zeitraum von mehreren Jahren mit selbst berichteter körperlicher Aktivität assoziiert waren. Im ersten und fünften Jahr war das körperliche Training mit einem positiveren psychologischen Profil verbunden. Höhere Levels an körperlicher Aktivität waren mit niedrigeren Depressionsraten assoziiert. Zusätzlich war eine Zunahme der körperlichen Aktivität zwischen dem ersten und dem fünften Jahr mit einem erhöhten Wohlbefinden verbunden (Foyert et al., 1995, zitiert nach Baker \& Brownell, 2000). Die Stimmungsaufhellung durch körperliche Aktivität verdient hinsichtlich des Zusammenhangs von Training und Gewichtskontrolle weitere Aufmerksamkeit. Es bedarf weiterer Studien, die die affektiven Symptome und ihre Verbindung zu 
emotional ausgelöstem Essen als Vermittler zwischen Aktivität und Gewichtsaufrechterhaltung untersuchen.

Es sollte weiterhin auf die Rolle der therapeutischen Zielsetzung als einer möglichen vermittelnden Variablen hinterfragt werden. Die Wahl von adäquaten Zielen hat einen großen Einfluss auf die Motivation und das Durchhaltevermögen. Depressive Menschen neigen dazu zu hohe Ziele zu setzen, welche bzgl. der Gewichtsreduktion zum Versagen und zur Entmutigung führen können (Baker \& Brownell, 2000).

Die Beziehung zwischen körperlichem Training und Stimmung kann eine wichtige Auswirkung auf die Rückfallquote haben. Emotionale Belastungen einschließlich Depression und Angststörungen sind mit Rückfällen assoziiert. Körperliches Training kann durch die positiven Veränderungen der psychologischen Zustände als eine Präventionsstrategie einem Rückfall entgegenwirken (Baker \& Brownell, 2000).

\subsubsection{Körperbild}

Das Körperbild ist eine weitere psychologische Variable, die wahrscheinlich positiv durch die körperliche Aktivität beeinflusst wird. Das Körperbild kann die Gewichtskontrolle sowie Einstellungen und Verhaltensweisen beeinflussen. Eine Person mit einem negativen Körperbild fühlt sich extrem unzufrieden mit ihrer Körperform und dem Körpergewicht. Das kann zu einer Frustration führen, wenn man die moderaten Gewichtsabnahmen, die die meisten Programme erreichen, bedenkt. Dieses wiederum kann zu einer negativen Stimmung, Resignation und letztlich zu einem Rückfall führen.

Überraschenderweise gibt es sehr wenige Studien zu den Effekten der körperlichen Aktivität auf das Körperbild, insbesondere im Rahmen eines Behandlungsprogramms der Adipositas. Hinweise darauf, dass das körperliche Training die Gefühle der Menschen über ihren Körper beeinflusst, lassen sich jedoch finden.

Mehrere Studien an normalgewichtigen Stichproben, zeigen wie körperliche Aktivität die Körperwahrnehmung positiv beeinflussen kann (Baker \& Brownell, 2000).

Körperliches Training bei Menschen mit Adipositas ist verbunden mit Veränderungen, die zu einem besseren Körperbild führen können. Dieses sind Veränderungen der körperlichen Umfänge (z.B. Abdomen und Hüfte). Wie sich bei der Untersuchung von Wood, Stefanick, Williams \& Haskell (1991) zeigte, können diese körperlichen Veränderungen zu einer Modifikation des Selbstbildes und des 
Selbstvertrauens führen. Diese positiven Effekte im Körperbild können einer Entmutigung und Resignation, wie oben beschrieben, vorbeugen.

Ein negatives Körperbild kann bei übergewichtigen Menschen eine Barriere für das körperliche Training sein. Menschen beschreiben häufig, ein unfreies, gehemmtes Gefühl in ihrer Trainingskleidung wahrzunehmen. Wenn sie in der Öffentlichkeit trainieren, haben sie Sorge, dass von anderen negative Äußerungen über sie gemacht werden. Verbesserungen des Körperbildes, die auf die körperliche Aktivität zurückzuführen sind, können verstärkend sein und zu einer langfristigen Aufrechterhaltung des Trainings führen. Sie können das Vertrauen in die eigene Fähigkeit, positive Veränderungen in Bezug auf die Figur oder das Gewicht zu machen, stärken.

Auf die Notwendigkeit nach mehr Forschung über den Einfluss der körperlichen Aktivität auf das Körperbild und, ob dieses dann auf eine verbesserte Aufrechthaltung der Gewichtskontrolle übertragen werden kann, sei an dieser Stelle deutlich hingewiesen (Baker \& Brownell, 2000).

\subsubsection{Selbstwertgefühl und Selbstwirksamkeit}

Selbstwertgefühl und Selbstwirksamkeit sind weitere psychosoziale Faktoren, auf die das Verhältnis von körperlichem Training und Gewichtskontrolle Einfluss nehmen kann. In der Übersichtsarbeit von Plante und Rodin (1990) wird Selbstwertgefühl als eine Folgevariable der körperlichen Aktivität und der psychischen Funktionsfähigkeit eingeschlossen. Alle relevanten Studien zeigen eine positive Beziehung zwischen körperlichem Training und Selbstwertgefühl. Menschen, die regelmäßig trainieren, haben ein besseres Selbstwertgefühl als diejenigen, die nicht trainieren. Auch wenn ihre Gewichtsabnahme geringfügig bleibt, sind Personen, die regelmäßig trainieren, stolz auf die Verbesserung ihrer Leistungsfähigkeit, was wiederum ihr Selbstwertgefühl verbessert (Mannix et al., 1999; Johnson, 2004). Wird der Begriff Selbstwertgefühl gebraucht, um die Selbsteinschätzung zu bezeichnen, dann scheint es, dass das körperliche Training zu einem global positiveren Selbstbild führen kann. Wie die Stimmungsaufhellung und die Steigerung des Wohlbefindens können Verbesserungen des Selbstwertgefühls zu einem positiveren psychologischen Klima führen, in dem Lifestyleänderungen ermöglicht werden. Ein verbessertes Selbstwertgefühl ermöglicht einem Menschen, sich selbst als eine Person zu sehen, die in der Lage ist, positive Veränderungen zu unternehmen und diese beizubehalten. Eine 
verstärkte Selbstwirksamkeit kann so zu einem gesteigerten Vertrauen in die eigenen Fähig-keiten führen.

„Beliefs in one's capabilities to organize and execute the courses of action required to manage prospective situations . . . . Efficacy beliefs influence how people think, feel, motivate themselves and act (Bandura, 1995, p. 2, zitiert nach Baker \& Brownell, 2000, p. 324).

Die Selbstwirksamkeit ist als wichtige Variable $\mathrm{zu}$ berücksichtigen, um die Beziehung zwischen körperlichem Training und Gewichtseinhaltung zu verstehen, da sie eine Variable ist, die in der Erklärung der gesunden Verhaltensweise (health behavior ) umfassend angewendet wird.

Es gibt aussagekräftige Forschung, die darauf hinweist, dass wahrgenommene Selbstwirksamkeit mit Verhaltensplänen und gesundheitsfördernden Verhaltensänderungen assoziiert wird. Selbstwirksamkeitsüberzeugungen beeinflussen kognitive Konstruktionen wie die Handlungspläne. Sie helfen beispielsweise bei der Visualisierung von Szenarios, die zum Erreichen der Ziele führen können. Menschen mit einem ausgeprägten Sinn für Wirksamkeit (Efficacy) können sich Erfolg vorstellen, während Personen, die an Ihrer Selbstwirksamkeit zweifeln, den Misserfolg antizipieren und ihre Aufmerksamkeit auf Dinge richten, die falsch verlaufen können.

In dem Kontext des körperlichen Trainings und der Gewichtskontrolle kann sowohl die Bewegungsaktivität als auch das Essverhalten zu einer erhöhten Selbstwirksamkeit führen (Baker \& Brownell, 2000).

Es gibt wenige Studien, die die Effekte der Teilnahme an sportlichen Aktivitäten auf die Selbstwirksamkeit direkt untersuchen. Korrelationsstudien weisen darauf hin, dass die Teilnehmer, die regelmäßig am Training teilnehmen, ein stärkeres Empfinden von Selbstwirksamkeit in Bezug auf Bewegung haben. Es ist jedoch sehr wahrscheinlich, dass diese Personen schon vor der Intervention stärkere Überzeugungen in Bezug auf das Aktivsein haben. Prospektive Studien sind nötig, die die ursprünglichen Werte der Selbstwirksamkeit untersuchen.

Eine derartige Studie untersuchte die Erwartungen an die Wirksamkeit von körperlicher Aktivität als „Outcome-Variable“ eines Berwegungsprogramms für Männer und Frauen mittleren Alters. Es wurde ein Anstieg der Selbstwirksamkeit sowohl durch die kurzzeitige als auch durch die langandauernde Teilnahme belegt. (McAuley, Bane \& Mihalko, 1995, zitiert nach Baker \& Brownell 2000). Sørensen, 
Anderssen, Hjerman, Holme \& Ursin (1997) untersuchten die Effekte auf eine Diätgruppe, einer Trainingsgruppe, einer Gruppe, die eine Kombination aus Diät und Training erhielt, und einer Kontrollgruppe (keine Intervention) hinsichtlich der Körperwahrnehmung und des körperlichen Könnens (Physical Mastery). Das Trainingsprogramm dauerte ein Jahr. Die Ergebnisse zeigten, dass das körperliche Training zu einer positiveren Selbstwahrnehmung bzgl. des körperlichen Könnens und der Fähigkeiten führte (Sørensen et al., 1997).

Es ist einleuchtend, dass Wirksamkeitserwartungen für diätetische Maßnahmen und körperliches Training in Verbindung stehen. Die Zunahme an Selbstwirksamkeit durch das körperliche Training kann die Selbstwirksamkeit bzgl. des Essverhaltens und der Gewichtsabnahme beeinflussen.

In diesem Sinne findet die o.g. Studie von Foreyt et al. (1995), dass bei adipösen Teilnehmern höhere Level an sportlichen Aktivitäten mit einer verbesserten Selbstwirksamkeit bzgl. ihres Essverhaltens verbunden sind. Darüber hinaus sind Steigerungen des körperlichen Trainings signifikant mit Verbesserungen der Selbstwirksamkeit des Essverhaltens verknüpft (Foyert et al., 1995).

\subsubsection{Bewältigungsstrategien}

Das körperliche Training kann als eine hilfreiche Bewältigungsstrategie in dem Prozess der verhaltensfördernden Änderungen und deren Aufrechterhaltung dienen. Die Verfügbarkeit von zuverlässigen Bewältigungsstrategien ist besonders wichtig für die Prophylaxe von Rückfällen während der Einhaltungsphase einer Verhaltensänderung.

Bewältigungsstrategie kann begrifflich als der Prozess einer Reaktion, mit stressvollen Situationen oder Ereignissen umzugehen, gesehen werden. Es bedeutet „einer Sache Herr zu werden“, „,einer Situation gewachsen zu sein“.

Bewältigungsstrategien können auf unterschiedlichen Ebenen angewendet werden. Problemorientierte „Copingstrategien“ führen zu Handlungen, die zur Lösung eines Problems führen, oder zur Suche nach Informationen, die zur Lösung des Problems beitragen können. Bei emotionsorientierten Copingstrategien geht es darum, Wege zu suchen, um die negativen emotionalen Reaktionen auf Stress abzubauen. 
Nach Long (1993) wirkt körperliche Aktivität wie eine emotionsorientierte Strategie, als ein Mittel zur Bewältigung der emotionalen Reaktionen des Stresses, vielleicht durch Ablenkung oder Entspannung. Für Personen, die als Reaktion auf negative Ereignisse übermäßig essen oder Essanfälle haben, kann körperliches Training als eine wichtige alternative Aktivität dienen (Baker \& Brownell, 2000).

\subsubsection{Spekulativer Charakter der psychologischen Mechanismen}

Mannix et al., (1999) fassen alle o.g. psychologischen Wirkungen des körperlichen Trainings als äußerst heilsam und nützlich für Menschen mit Adipositas, die an Gewicht verlieren möchten, zusammen. Forschungsergebnisse aus dem Bereich diätetischer Maßnahmen bestätigen die landläufig bekannte Erfahrung, dass restriktives Essverhalten zu Depression, Angst, Erschöpfung und Reizbarkeit führt. Bei der Hälfte aller Diätetiker zeigen sich nach (Mannix et al., 1999) diese Stimmungs-veränderungen.

Personen mit Adipositas, die an Gewicht verlieren möchten, kann körperliches Training bei der Stressreduktion helfen. Es verbessert die Selbstwerteinschätzung und das Selbstkonzept, zwei Bereiche, die nach vielen Jahren Adipositas häufig hochgradig herabgesetzt sind.

Das zunehmende Gefühl von persönlicher Kontrolle und Leistungsfähigkeit, gewonnen durch das körperliche Training, kann indirekt Essgewohnheiten beeinflussen und aufgrund von kleinen Erfolgserlebnissen weitere positive Lifestyleveränderungen herbeiführen.

In der Praxis sollen Mediziner wie andere Fachleute den Patienten die Bedeutung der körperlichen Aktivität und des Trainings für eine verbesserte psychische Funktionsfähigkeit vermitteln. Die Möglichkeit durch körperliche Aktivität und Training, ein unmittelbares Gefühl des Wohlbefindens auszulösen, sollte weder unterschätzt noch übersehen werden (Mannix et al., 1999).

Baker und Brownell (2000) gehen davon aus, dass körperliches Training und Gewichtskontrolle teilweise mit den psychologischen Mechanismen verknüpft sind. Sie bemängeln jedoch, dass diese Zusammenhänge zur Zeit großteils hypothetisch sind.

Die Forschung auf dem Gebiet des körperlichen Trainings und der Gewichtsstabilisierung hat allgemein die potentielle Rolle der psychologischen Variablen übersehen. Ein großes Hindernis für die Erfassung einer ursächlichen Rolle der 
psychologischen Variablen ist die Schwierigkeit, diese von den physiologischen Effekten des körperlichen Trainings zu trennen. Die psychologischen Effekte des körperlichen Trainings können entweder Resultate biologischer oder kognitiver Faktoren sein oder sie ergeben sich aus einer komplexen Wechselwirkung zwischen der Physiologie und der Psychologie.

Baker und Brownell (2000) betonen die besondere Bedeutung kognitiver Faktoren wie des Selbstwertgefühls und der Selbstwirksamkeit. Sie plädieren für eine aufgeschlossene Betrachtung der potentiellen Rolle der psychologischen Variablen in der Beziehung zwischen körperlicher Aktivität und Gewichtseinhaltung.

Abschließend ist es eindeutig, dass dadurch offene Forschungsfragen zu beantworten bleiben (Baker \& Brownell, 2000).

\subsection{Weitere gesundheitliche Effekte der körperlichen Aktivität bei Adipositas}

Obwohl körperliches Training kein Allheilmittel für die Behandlung der Adipositas ist, ist eine Effizienzbemessung, die nur auf die Gewichtsreduktion beschränkt ist, ungerecht und wahrscheinlich irreführend (Bensimhon et al., 2006).

Es stellt sich die Frage, ob körperliches Training und Adipositas einen Einfluss als Prädiktor auf die Mortalität haben. Es gibt aber keine Daten von randomisierten klinischen Studien, die die Beziehung zwischen körperlicher Aktivität und Mortalität untersuchen. Eine Fülle von Beobachtungshinweisen unterstützt jedoch die Annahme, dass kleine Mengen an regelmäßiger körperlicher Aktivität 20 bis $30 \%$ aller Ursachen, insbesondere kardiovaskuläre Mortalitätsraten, reduzieren können (Lee \& Skerrett, 2001, zitiert nach Bensimhon et al., 2006).

Als besonders nützlich ist hier die Aerobics Center Longitudinal Study zu erwähnen. Diese Längsschnittstudie, die über 24 Jahre fast 26.000 Männer im Alter von 30-83 Jahren einschloss, untersuchte alle 8 Jahre die Gesundheitsvorteile von kardiorespiratorischer Fitness und Körperausmaßen. Die medizinische Untersuchung beinhaltete anthropometrische Messungen, eine fahrradergometrische Belastungsuntersuchung als Maximaltest und andere klinische Messungen. Es konnte gezeigt werden, dass hohe Level an kardiovaskulärer Fitness Schutz gegen kardiovaskuläre Krankheiten und jede Mortalitätsursache brachten. Dies galt für normal- und übergewichtige Männer. Körperlich leistungsfähige Männer, die adipös waren, hatten substantiell niedrigere Sterblichkeitsraten, als normalgewichtige Männer, die nicht 
körperlich fit waren. Niedrige Fitness ist ein wichtigerer Prädiktor für Mortalität als die Fettleibigkeit (Blair \& Holder, 2002; Blair \& Leermakers, 2004).

Es gibt eine Reihe von gesundheitsförderlichen Auswirkungen regelmäßiger körperlicher Aktivität auf Folge- und Begleiterkrankungen der Adipositas. Solche Effekte zeigen sich in einer Steigerung der Herz- Kreislaufsteigerungsfähigkeit und Lungenfunktion und einer Verbesserung von Stoffwechselprozessen. Einige dieser Effekte sind sogar unabhängig von einer Gewichtsreduktion (Hauner \& Berg, 2000; Vögele 2003; Fogelholm, Stallknecht \& Van Baak, 2006). Wenn jedoch eine Gewichtsreduktion (von 5 oder $10 \%$ des Ausgangsgewichts) in Betracht gezogen wird, können substantielle Verbesserungen bzgl. der Risikofaktoren für kardiovaskuläre Erkrankungen und Diabetes erzielt werden. Dieses kann zu einer Herabsetzung oder Einstellung der Medikation führen (Bensimhon et al., 2006).

Abschließend kann man sagen, dass eine Reihe von Studien existieren, die auf die gewichtsunabhängigen positiven Effekte der körperlichen Aktivität wie Schlafapnoe und die Inzidenzraten von Darmkrebs, Brustkrebs, Osteoarthritis und Osteoporose hinweisen (Bensimhon et al., 2006).

Die wichtigsten somatischen Effekte des körperlichen Trainings auf die Adipositas sollen in ihrer physiologischen Wirkungsweise kurz dargestellt werden.

\subsubsection{Diabetes mellitus und Glykoseintoleranz}

Körperliche Aktivität steigert die Insulinwirkung. Diese trainingsinduzierte Wirkung hat klinisch eine große Bedeutung, da beim Typ-2-Diabetes und beim metabolischen Syndrom eine Insulinresistenz besteht. Durch körperliches Training wird die Glykoseaufnahme um 20-40 \% gesteigert. Wenn die Insulinsensitivität steigt, wird die Glykosetoleranz erhöht. Vermehrte Bewegung und eine Umstellung der Ernährung können eine präventive Funktion haben und den Diabetes kontrollieren (Mannix et al. 1999; Wirth, 2003; Fogelholm, et al., 2006).

Des Weiteren stellt man bei Typ-2-Diabetikern fest, dass Blutglykose und $\mathrm{HbA}_{1}$ abhängig von Umfang und Intensität des Trainings sinken. Die Kombination von Bewegung und Diät reduziert die Blutglykose, Hämoglobin $\left(\mathrm{HbA}_{1}\right)$ und den Insulinbedarf. Ein effektives Ausdauertraining senkt den $\mathrm{HbA}_{1 \mathrm{c}}-$ Wert wie auch eine Therapie mit oralen Antidiabetika oder Insulin (Wirth, 2003). 


\subsubsection{Verbesserungen des Lipidprofils}

Bei Menschen mit Adipositas sind Lipide und Lipoproteine oft verändert. Körperliche Aktivität kann eine Verbesserung des Lipidprofils erzielen (Mannix et al. 1999; Wirth, 2003; Vögele, 2003).

Insbesondere Triglyzeride, HDL-Cholesterin und small-dense LDL können durch die körperliche Aktivität verändert werden. Triglyzeride werden durch Ausdauertraining um 10-40\% gesenkt, das HDL-Cholesterin steigt zwischen 5 und $20 \%$, je nach Umfang und Intensität des Trainings. Auch die atherogenen kleinen, dichten LDLPartikel werden durch Training in Kombination mit einer Reduktionskost modifiziert (Wirth, 2003; Fogelholm et al., 2006).

\subsubsection{Abnahme von Blutdruck}

Körperliches Training führt $\mathrm{zu}$ einer Blutdrucksenkung, d.h. der systolische Blutdruck nimmt deutlich ab. Davon können übergewichtige Patienten sogar auch ohne Gewichtsabnahme profitieren (Vögele, 2003).

Da das körperliche Training auch die Herzfrequenz senkt, betont Wirth (2003), wie deutlich das so genannte Doppelprodukt aus Herzfrequenz $\times$ systolischem Blutdruck reduziert wird. Diese Anpassung ist vor allem für Patienten mit einer koronaren Herzkrankheit wichtig, da das Doppelprodukt eine wichtige Determinante des myokardialen Sauerstoffverbrauches ist (Wirth, 2003, S. 126).

\subsubsection{Verbesserung des Herz- Kreislaufsystems}

Ein körperliches Training verbessert die Leistungsfähigkeit des Herzens und die Ökonomie der Herzarbeit über muskuläre und neuronale Adaptationen. Diese kardiovaskulären Trainingseffekte (z.B. Erhöhung des Sauerstoffaufnahmevermögens, Senkung des Ruhepulses, Erhöhung des Schlagvolumens usw.) sind bei übergewichtigen, wenn sie regelmäßig trainieren, ähnlich wie bei normalgewichtigen Personen (Mannix et al., 1999; Vögele, 2003).

Nach dem Evidenzreport des „National Heart, Lung and Blood Institute (NHLBI)“ verbessert körperliche Aktivität bei übergewichtigen und adipösen Erwachsenen die kardio-respiratorische Fitness unabhängig vom Gewichtsverlust. Dieser Effekt ist nach NHLBI, 1998 bei einem Evidenz-Grad A nachgewiesen. Die vermehrte Bewegung in Verbindung mit einer Reduktionskost verringert die kardiovaskulären 
Risikofaktoren deutlich, so dass mit einer Abnahme der koronaren Herzerkrankungsinzidenz zu rechnen ist (Wirth, 2003).

\subsection{Verordnung der körperlich-sportlichen Aktivität bei der Behandlung von Adipositas}

Die Bestimmung der optimalen Dauer, Intensität und Art des Trainings, welches benötigt wird, um eine Gewichtsreduktion zu fördern, kardiovaskuläre Risikofaktoren $\mathrm{zu}$ verbessern und die Mortalitätsrate $\mathrm{zu}$ minimieren, bleibt eine große Fragestellung der Forschung.

Gesundheitsempfehlungen hinsichtlich der Verordnung körperlichen Trainings sind in den letzten Jahren im Wandel. Während früher Empfehlungen primär auf die Förderungen der funktionellen Kapazitäten gerichtet waren, sind neuere an den Voraussetzungen körperlich-sportlicher Aktivität orientiert, die notwendig sind, um das Risiko chronischer Erkrankungen zu reduzieren (Woll \& Bös, 2004). Es muss erwähnt werden, dass diese Richtlinien aus Gesundheitsorganisationen des nordamerikanischen Raumes stammen und so übernommen werden.

Nach den Empfehlungen des "Center for Disease Control and Prevention" und dem "American College of Sports Medicine (ACSM)" (1995) und dem U.S. Department of Health and Human Services (1996) sollten Menschen jeden Alters ,accumulate 30 minutes or more of moderate intensity physical activity on most preferably all days, of the week" (U.S. Department of Health and Human Services 1996, S. 28).

Wichtige Merkmale dieser Empfehlungen sind die Ausdehnung der Aktivität auf alle Wochentage, die nicht kontinuierliche, also unterbrochene Akkumulation der körperlichen Aktivität und der Focus auf Aktivitäten mit einer moderaten Intensität. Unter „moderater Intensität“ wird für die Mehrheit der passiven Erwachsenen jede Aktivität eingeschätzt, die mit einer Intensität durchgeführt werden kann, die in etwa dem zügigeren Gehen (brisk walking) entspricht. Nach Blair \& Leermakers (2004) heißt es: Gehen von 3,2 km in 30-40 min oder Gehen mit einem Schritttempo von 912 min pro km. Ein dreimal tägliches zehnminütiges Gehen würde die Empfehlungen für die tägliche Aktivität erfüllen (Blair \& Leermakers, 2004).

Ein weiterer Vorteil der Richtlinien hinsichtlich des Begriffs der moderaten körperlichen Aktivität ist die Erweiterung der Sicht auf sportlich-körperlichen Aktivitäten. Der Begriff beinhaltet alle Formen von Alltagsaktivitäten, die zu einer 
gesundheitlich wirksamen Steigerung des Energieverbrauchs führen. Solche Aktivitäten können während der Berufs- oder Hausarbeit und als Mittel des Transports stattfinden.

Damit eine Aktivität wirkungsvoll ist, muss sie nicht hoch intensiv sein, an einem speziellen Ort stattfinden (Fitnessraum, Tennisplatz) oder zeitlich zusammenhängend in einer Trainingseinheit absolviert werden. Dieses neues Denken über das körperliche Training führte zu dem Konzept der „lifestyle physical activity“, in dem nicht aktive Personen ermutigt werden, diese gemäßigten körperlichen Aktivitäten in den Alltag zu integrieren und besser aufrechtzuerhalten (Blair \& Leermakers, 2004; Woll \& Bös, 2004).

Diese oben genannten Empfehlungen lassen sich wie folgt auf die Adipositas übertragen:

- es besteht ein Dosis- Wirkung- Zusammenhang zwischen dem Volumen des körperlichen Trainings und jeder Mortalitätsursache

- $\quad$ eine einwöchige Energieausgabe von mindest $1000 \mathrm{kcal} / \mathrm{w}$ (das entspricht 30 min. 3,2 km schnelles Walking 5-mal die Woche) ist notwendig, um signifikant die Mortalitätsraten bzw. Risikofaktoren um 20-30 \% zu reduzieren (Lee \& Skerrett, 2001, zitiert nach Bensimhon et al., 2006).

Einen ähnlichen Zusammenhang von Dosis und Wirkung findet man zwischen dem Ausmaß des körperlichen Trainings und der Modifikation von multiplen kardiovaskulären Risikofaktoren (Bensimhon et al., 2006).

Ein Trainingsmaß von 150-200 $\mathrm{min} / \mathrm{wchtl}$. (25-30 min/tgl.) von moderater körperlicher Aktivität kann die kardiovaskuläre Leistungsfähigkeit wie auch die Insulinsensitivität (bei untrainierten Personen) verbessern, führt aber zu keinem besonderen Ergebnis hinsichtlich des Gewichts, der Körperzusammensetzung und Fettverteilung bei den meisten Personen (Fogelholm et al., 2006).

Die oben genannten Aktivitätslevels gelten als anfängliche Basis für Bewegungsinterventionen, die auf untrainierte übergewichtige Personen zugeschnitten sind (Jakicic, 2003).

Nach Hauner \& Berg (2000) scheint eine körperliche Belastung hoher Intensität bei adipösen Menschen keine entscheidenden Vorteile gegenüber einer solchen von niedriger oder mittlerer Intensität zu bieten. „Die günstigen Effekte auf Körperzusammensetzung und Risikofaktoren sind kaum größer, die Akzeptanz einer hohen 
Belastung dagegen deutlich geringer" (Hauner \& Berg, 2000, S. 773). Die Autoren fassen die wichtigsten Vor- und Nachteile einer regelmäßigen körperlichen Belastung niedriger Intensität gegenüber hoher Intensität zusammen:

\section{Vorteile:}

- lässt sich bei stark adipösen Patienten leichter praktisch umsetzen (besonders zu Beginn der Gewichtsreduktion)

- lässt sich häufig (zum Beispiel täglich) durchführen

- wird besser toleriert und erreicht eine bessere Compliance

- die Verletzungsgefahr ist deutlich geringer

- größere medizinische Voruntersuchungen sind nicht notwendig

- die günstigen Veränderungen von BMI und Körperzusammensetzung sind annähernd gleich

- es werden vorzugsweise im Fettgewebe gespeicherte Fettsäuren mobilisiert und oxidiert

\section{Nachteile:}

- der Gewinn an körperlicher (kardiorespiratorischer) Fitness ist geringer

- eventuell geringerer Gewinn an Lebenserwartung (?)

(Hauner \& Berg, 2000, S. 773).

Wenn es jedoch um eine Gewichtsreduktion oder -einhaltung geht, ist mehr körperliches Training erforderlich als benötigt wird, um kardiovaskuläre Fitness aufzubauen (Bensimhon et al., 2006; Jakicic, 2003).

„The recommended amount of physical activity for the management of obesity depends on several issues such as the magnitude of desire outcome, recent history of weight change and dietary habits" (Fogelholm et al., 2006, p. 21).

Mehrere Studien weisen darauf hin, dass für eine langfristige Gewichtskontrolle das Ausmaß an körperlicher Aktivität höher sein soll, als es die Richtlinien des amerikanischen Instituts für öffentliche Gesundheit (U.S. Department of Health and Human Services) vorschlagen. In einer amerikanischen Untersuchung, dem „National Weight Control Registry“ (Wing \& Phelan, 2005), verbrauchten 52 \% der Personen, die ihr Gewicht erfolgreich gehalten haben, mehr als 1000 kcal in der Woche durch Bewegung. Männer berichteten im Mittelwert von einem wöchent- 
lichen Kalorienverbrauch von 3293 kcal und Frauen von 2545 kcal. Das entspricht 60 bis 80 Minuten moderater Aktivität, wie täglichem zügigem Gehen (Wing \& Phelan, 2005).

Andere Studien kommen zu ähnlichen Ergebnissen. Um eine Gewichtsreduktion erfolgreich aufrechtzuerhalten, ist ein täglich 80-minütiges moderates oder 35minütiges sportliches Ausdauertraining, ein höherer täglicher Kalorienverbrauch durch Alltagsarbeiten oder ein Minimum von 280 min körperlichen Trainings wöchentlich erforderlich (Jakicic, 2003).

Die „International Association for the Study of Obesity“ (IASO) gab in Reaktion auf die Gesundheitsempfehlungen von 1996 und beeinflusst durch neuere Forschungsergebnisse auf einer „Consensus Conference“ im Jahr 2003 folgende Erklärung ab:

„... for preventing weight gain or regain this guideline (CDC, ACSM 1995) is likely to be insufficient for many individuals in the current environment. There is compelling evidence that prevention of weight regain in formerly obese individuals requires 60-90 minutes of moderate intensity activity or lesser amounts of vigorous intensity activity. Although definitive data are lacking, it seems likely that moderate intensity activity of approximately 45 to 60 minutes per day, or 1.7 PAL (Physical Activity Level) is required to prevent the transition to overweight or obesity" (Saris et al., 2003 p. 111).

\subsection{Inhalte und Methoden des Trainings bei Adipositas}

Sport- und bewegungstherapeutische Interventionen bei Adipositas zeichnen sich durch eine Vielfalt an Methoden und Inhalten aus. Es gibt ganz unterschiedliche Trainingsprogramme, und auch die praxisorientierten Bücher sowie Broschüren der Krankenkassen geben eher ein unübersichtliches, uneinheitliches Bild hinsichtlich praktischer Tipps, Empfehlungen und der beschriebenen Methodik und Didaktik des Sports und der Bewegungstherapie bei Adipositas.

Bei den landläufigen Angeboten wurde bislang wenig auf die psychosozialen Gründe und deren Bedeutung für die Bewegungsangebote wie auch auf die aktuellen psychosozialen Einschränkungen der Menschen mit Adipositas an sich geachtet.

In der Adipositasliteratur werden vorwiegend die zwei Trainingsformen Ausdauertraining und Krafttraining diskutiert. 
Eine immer wichtigere Rolle in der Sporttherapie bei Adipositas stellt in den letzten Jahren der „Lifestyle Physical Activity“-Ansatz dar.

Letztlich findet sich in den „Indikationskatalogen Sporttherapie“ von Schüle und Schnieders (2004) ein adipositasspezifischer Katalog. Dieser berücksichtigt Schädigungen, Beeinträchtigungen sowie Partizipation unter dem Aspekt sporttherapeutischer Zielsetzungen und Maßnahmen. Im Folgenden werden gängige Interventionen dargestellt und in Bezug auf die wissenschaftliche Bedeutung diskutiert.

\subsubsection{Ausdauersport}

Die Formen des Ausdauersports sind die am häufigsten untersuchten Trainingsformen bei einer Gewichtsreduktion. Walking, Aerobic, Fahrradergometer erweisen sich als effektives Ausdauertraining zur Verbesserung der kardiorespiratorischen Fitness mit ihren positiven gesundheitsfördernden Effekten.

Im Bezug auf Adipositas spielt Ausdauertraining eine wichtige Rolle. Ausdauertraining hat die besten Effekte auf die Herzkreislaufparameter. Die mittlere Intensität der Belastung ermöglicht auch Untrainierten, die für diese Effekte und einen Kalorienverbrauch notwendigen Trainingszeiten zu erreichen. Ausdauersport kann auch für Übergewichtige weitgehend Gelenk schonend ausgeführt werden. Der in der Literatur noch oft $\mathrm{zu}$ findende Vorteil der vermehrten Fettverbrennung ist nicht relevant. Bei jeder körperlichen Belastung werden alle Komponenten der physiologischen Energiebereitstellung aktiviert. Auch wenn es stimmt, dass im Ausdauertraining im Vergleich zum Krafttraining der Fettstoffwechsel etwas aktiver ist, hat dieses keinen Einfluss auf eine vermehrte Reduktion des Körperfetts, wie es von der Fitnessindustrie postuliert wird. Gewichtsreduktion entsteht einzig durch die negative Energiebilanz, wie die Energie verbraucht wird, spielt hier keine Rolle, allein die Menge der verbrauchten Energie im Verhältnis zur Energieaufnahme zählt (Huber, 2007).

Studien aus dem amerikanischen Raum zeigen, dass Walking sich als die populärste Form des Ausdauertrainings bei den Übergewichtigen durchgesetzt hat (Jakicic, 2003). 


\subsubsection{Krafttraining}

Das Krafttraining gewann in der letzten Dekade an Bedeutung. Verluste der Muskelmasse und die Senkung des Grundumsatzes während einer Gewichtsreduktion sind unerwünschte Auswirkungen. Man nahm an, diese durch gezieltes Krafttraining verhindern zu können. Studien weisen darauf hin, dass die Hinzufügung von Krafttraining zu Ausdauertraining und Diät im Vergleich zu reiner Diät oder zu einer Kombination von Diät und Ausdauertraining den Verlust der mageren Körpermasse minimiert. Der absolute Gewichtsverlust bleibt bei einer derartigen Kombination allerdings üblicherweise unverändert oder ist sogar leicht abgeschwächt (Bensimhon et al., 2006; Jakicic, 2003). Bei Perioden mit stärkeren kalorischen Restriktionen kommt es durch das Krafttraining sogar zu einem Zuwachs an Muskelquerschnittsfläche, ohne dass dieses die Abnahme der mageren Körpermasse mindert.

Krafttraining scheint die Senkung des Grundumsatzes im Vergleich mit Ausdauertraining während einer Gewichtsabnahme nicht signifikant $\mathrm{zu}$ verkleinern (Bensimhon et al., 2006; Jakicic, 2003).

Abschließend kann gesagt werden, dass Krafttraining die Gewichtsreduktion im Vergleich zu Ausdauertrainingsformen nicht signifikant verbessert (Votruba et al., 2000; Jakicic, 2003).

Methodologische Probleme der Untersuchungen über das Krafttraining sind jedoch eindeutig. So gibt es nur wenige Langzeitstudien und die untersuchten Krafttrainingsinterventionen der meisten Studien dauern weniger als sechs Monate an (Jakicic, 2003).

Die Integration eines Krafttrainings in die Präventions- und Behandlungsprogramme für übergewichtige und adipöse Menschen ist aufgrund der bekannten Wirkungen des Krafttrainings auf die Muskelkraft zu empfehlen. Muskelkraft kann die Funktion und die Fähigkeit der übergewichtigen bzw. adipösen Personen verbessern, um ihre alltäglichen Bewegungen auszuführen. Beispielsweise kann die Fähigkeit, das Eigengewicht zu tragen, dazu führen, einen leichteren Zugang zu körperlichen Aktivitäten zu bekommen. Dieses fördert die Funktionsfähigkeit, aktiviert und kann so zu einer Verbesserung der Lebensqualität führen.

Nicht die Gewichtsreduktion, sondern die o.g. Gründe führen zu der Empfehlung, Krafttraining in die Behandlung von Adipositas zu integrieren (Graf \& Predel, 2005; Jakicic \& Otto, 2005; Fogelholm et al., 2006). 
Ergebnisse mehrerer Studien lassen darauf schließen, dass im Rahmen von Gewichtsreduktionsprogrammen keiner der beiden Trainingsarten der Vorzug gegeben wird. Vielmehr wird empfohlen, Ausdauertraining und Kraftsportarten zu kombinieren, da beide Trainingsformen spezifische Vorteile aufweisen (Vögele, 2003; Jakicic, 2003; Fogelholm et al., 2006).

Trotz der Menge anderer Sportarten bzw. Spielsportarten gibt es wenige Daten über ihren Einfluss auf eine Gewichtsreduktion oder -einhaltung (Jakicic, 2003).

Die Einteilung der Sportarten in geeignet, bedingt geeignet und ungeeignet nach Wirth (2003) sollte doch mit der Aussage „es gibt keine "richtigen" oder "falschen" Sportarten für Übergewichtige“ relativiert werden. Vielmehr soll darauf geachtet werden, dass die empfohlenen Aktivitäten den Teilnehmern Spaß machen, sicher, effektiv und zugänglich sind. Aufgrund der spezifischen Voraussetzungen bei Adipösen können einige Empfehlungen bzgl. der Sportarten formuliert werden (Wirth, 2003; Vögele, 2003).

Es folgt eine Auflistung wichtiger Aspekte der Adipositas und ihrer Folgen, die bei der Auswahl von körperlich- sportlichen Aktivitäten berücksichtigt werden sollen:

- das Tragen des Eigenkörpergewichtes,

- Gelenkprobleme (Gonarthrose, andere degenerative Veränderungen der unteren Extremitäten),

- eingeschränkte Motorik durch die Körperfülle (Weichteilsperre),

- Koordinationsschwierigkeiten bzw. Gleichgewichtsunsicherheit,

- mangelnde Leistungsfähigkeit,

- Motivationsprobleme,

- evtl. eine daraus resultierende erhöhte Verletzungsgefahr (Mannix et al., 1999; Wirth, 2003; Vögele, 2003; Kuhn, 2001).

\subsection{3 „Lifestyle Physical Activity“ versus Bewegungsprogramme}

In der deutschsprachigen Literatur wird der amerikanische Begriff der „Lifestyle Physical Activity“ mit „Alltagsaktivitäten“ (Vögele, 2003) und „LebensstilAktivitäten“ (Fuchs, 2003) übersetzt. Die vorliegende Arbeit favorisiert den amerikanischen Begriff und übernimmt ihn als Fachbegriff. 
Eine zusätzliche Alternative $\mathrm{zu}$ den traditionellen strukturierten Bewegungsprogrammen stellen die Interventionen dar, die auf eine Änderung der „Lifestyle Physical Activity“ abzielen. Traditionelle Richtlinien heben bei ihren Empfehlungen trainingswissenschaftliche Parameter wie die Häufigkeit, Intensität, Dauer und Art hervor. Es werden moderat starke Belastungen mit einer Dauer von 20-40 min, bei $70 \%$ der maximalen Herzfrequenz an 3-5 Tage der Woche empfohlen. So wirkungsvoll diese Empfehlungen auch sein mögen, sie verfehlen in der Regel ihr Ziel, die meisten Erwachsenen dazu zu motivieren, in dieser Art habituell körperlich aktiv zu werden. Die fehlende Umsetzung der Empfehlungen der körperlichsportlichen Aktivitäten zeigt, dass der traditionelle lineare Ansatz der gesundheitsfördernden Wirkungen nicht ausreichend ist, um den umwelt- und soziokulturellen Einflüssen mit der Folge von Inaktivität zu begegnen.

Hinzu kommt, dass einige der adipösen Menschen anfangs nicht leistungsfähig genug sind, um ein 30-minütiges kontinuierliches Training in einer Einheit zu realisieren (Pescatello \& VanHeest, 2000; Blair \& Holder, 2002).

Die Auswertung der bisherigen Forschung einschließlich neuester Studien führte zu einer geänderten Richtlinie körperlich-sportlicher Aktivität. Dunn et al. (1988) definierten den „Lifestyle Physical Activity“- Ansatz als die tägliche Akkumulation von mindestens 30 Minuten selbst ausgewählter, nicht verordneter Aktivitäten einschließlich Freizeitaktivitäten, Berufsarbeit und Hausarbeit. Diese müssen in ihrer Intensität moderat sein. Die Aktivitäten können geplant oder ungeplant, strukturiert oder unstrukturiert sein. Sie sollen Teile regelmäßig anfallender Aufgaben des Alltagslebens werden (Dunn et al., 1998). Ein Beispiel dafür ist, zu Fuß zu gehen anstatt motorisierte Alternativen wie z.B. Autos, Lifte und Rolltreppen zu benutzen. Inaktive Personen werden so ermutigt, körperliche Aktivität in ihren Alltag zu integrieren. Hierdurch soll die reduzierte tägliche Energieausgabe, die durch die Technisierung des modernen Leben verursacht wurde, ausgeglichen werden (Blair \& Holder, 2002; Wadden, Butryn \& Byrne, 2004; Jakicic \& Otto, 2005).

Es ist offensichtlich, dass die „Lifestyle Physical Activity“- Bewegungsprogramme von den Richtlinien des „Centers for Disease Control and Prevention“ und dem „American College of Sports Medicine (ACSM)“ aus dem Jahr 1995 beeinflusst sind und sich von den älteren Empfehlungen distanzieren.

Blair \& Holder (2002) betonen weitere Vorteile bei einer genaueren Erklärung des Ansatzes. Das Konzept hilft den Teilnehmern bei der Ansammlung der Alltags- 
aktivitäten kognitive Strategien und Verhaltensstrategien anzuwenden. Die Aktivitäten als Alltagsaktivitäten müssen nicht im engeren Sinne sportliche Handlungen sein. Der Ansatz soll Menschen helfen, die traditionelle Ansicht hinsichtlich eines sportlichen Trainings zu ändern. Die Teilnehmer sollen sich eine weitsichtige Definition aneignen, welche eine größere Auswahl an Optionen für die körperliche Aktivität anbietet (Blair \& Holder, 2002).

Das Konzept der „Lifestyle Physical Activity“ beruht auf dem „Transtheoretischen Modell der Verhaltensänderung“ von Prochaska und DiClemente (1983). Nach dieser Theorie ändern sich Personen nicht in einem gleichen Maß. Der Prozess der Änderung ist in unterschiedliche Abschnitte unterteilt. Jeder Abschnitt für sich weist besondere Merkmale auf. Eine Vielfalt kognitiver und behavioraler Strategien können angewendet werden, um die passende Intervention für die Bedürfnisse der Zielgruppe zu gestalten. Der Grad der Motivationsbereitschaft und andere populationsspezifische Charakteristika werden berücksichtigt.

Es sind jedoch auch andere Verfahren in das Konzept der „Lifestyle Physical Activity“ eingebaut. Zielsetzung (Goal-Setting), Selbstbeobachtung, und Problemlösungsverfahren (problem solving) in Hinblick auf die Hindernisse bei der Ausübung körperlicher Aktivitäten machen gemeinsam mit anderen traditionellen kognitiven Fertigkeiten und Verhaltensweisen das Gesamtkonzept aus (Blair \& Holder, 2002; Blair \& Leermakers, 2004).

Aufgrund der niedrigen Leistungsfähigkeit adipöser Patienten kann der Einsatz des „Lifestyle Physical Activity“ Konzepts ein durchführbarer Weg sein, um die vorgeschlagenen Aktivitätslevels zu erreichen. Es erlaubt den Teilnehmern sich ihre eigenen Bewegungsprogramme „maßzuschneidern“, eine Vielfalt mittelintensiver Aktivitäten einzuschließen und die kurzen Trainingseinheiten so zu sammeln, wie es die individuellen Lebensverhältnisse erlauben.

Das Konzept kann der erste Schritt hin zu einer Aktivierung für Patienten sein, die zunächst von einem ununterbrochenen sportlichen Ausdauertraining abgeschreckt sind. Das Konzept scheint einen langfristigen Effekt hinsichtlich der Partizipation an den körperlich-sportlichen Aktivitäten zu haben. Die Steigerung der Alltagsaktivitäten kann eine Brücke zur weiteren Teilnahme an anderen sportlichen Trainingsformen sein. Nach der Erfahrung der Selbstwirksamkeit, erreicht durch ein erfolgreiches „Lifestyle Physical Aktivity“- Programm kann eine Person körperlich 
und mental für die Herausforderung eines regelmäßigen sportlichen Trainings gestärkt sein (Franckowiak \& Andersen, 2003).

Wadden et al. (2004) bringen es auf den Punkt wenn sie schreiben:

„Initial findings, however, suggest that lifestyle activity is an ideal alternative for patients who report they hate to exercise" (Wadden et al., 2004, p. 156).

Bei der Evaluation des Konzeptes in den letzten Jahren zeichnet sich deutlich ab, dass „Lifestyle Physical Activity“ -Programme d.h. die Steigerung der Alltagsaktivität, einen ähnlich hohen gesundheitlichen Nutzen wie strukturierte Sportprogramme haben (Dunn et al., 1998). In zwei häufig erwähnten kontrollierten Studien von Dunn et al. (1999) und Andersen et al. (1999) wurden die Auswirkungen einer Steigerung der Alltagsaktivität mit denen strukturierter Bewegungsprogramme hinsichtlich Körpergewicht, Körperzusammensetzung, Risikofaktorenprofil und Fitness verglichen. Die günstigen Effekte auf diese Parameter waren bei beiden Strategien ähnlich. Die Tatsache, dass in der Studie von Dunn et al. (1999) „Project Active“ die Intervention keine Gewichtsverluste produzierte, überrascht nicht, da der Fokus der Intervention nicht auf Änderungen der Ernährungsgewohnheiten lag. In der Studie von Andersen et al. (1999) waren diätetische Maßnahmen (1200 kcal/tgl.) mit einbezogen. Teilnehmer beider Gruppen verloren in einem vergleichbaren Ausmaß an Gewicht. Während der 1-jährigen follow-up Periode nahmen die Teilnehmer des „Lifestyle Physical Activity“-Programms signifikant weniger an Gewicht zu, als die des strukturierten Bewegungsprogramms.

Diese Ergebnisse zeigen, dass ein „Lifestyle Physical Activity“-Programm im Vergleich mit einem strukturierten Bewegungsprogramm genauso effektiv, in der Unterstützung einer diätetischen Gewichtsreduktion und eventuell noch effektiver bezüglich einer langfristigen Gewichtserhaltung ist.

Obwohl die Forscher eine genaue Beschreibung der Inhalte der „Lifestyle Physical Activity“- Programme in den Studien angeben, sind die genaue Art, Dauer und Intensität der Alltagsaktivitäten, auf die sich die Teilnehmen eingelassen haben, nicht berichtet. Aus dem Grund bleibt es schwierig zu bestimmen, wie sich der Ansatz der „Lifestyle Physical Activity“ von traditionellen Ansätzen des körperlichen Trainings abgrenzt. Worin unterscheiden sich „Lifestyle Physical Activity“-Programme von anderen häuslichen Bewegungsprogrammen, die sich als ähnlich effektiv erwiesen haben? (Jakicic, 2003; Jakicic \& Otto, 2005). 
Trotz der bewiesenen Effektivität ist es nicht klar, ob die „Lifestyle Physical Activity“-Programme allein die signifikanten Verbesserungen hinsichtlich des Körpergewichts und andere, Gesundheitsparameter hervorrufen (Bensimhon et al., 2006). Weitere Studien sind nötig, um die Auswirkungen der spezifischen Formen und Muster der „Lifestyle Physical Activity“-Programme auf die o.g. Parameter bei den übergewichtigen Menschen zu verstehen (Jakicic, 2003; Jakicic \& Otto, 2005).

\subsubsection{Heimtraining versus klinische Bewegungsprogramme}

Die meisten Bewegungsprogramme werden traditionell unter Anleitung im Rahmen eines therapeutischen Settings angeboten. Die Vorteile derartiger Interventionen bestehen darin, dass der Patient in modernen Einrichtungen und von ausgebildetem Personal betreut wird. Die Gruppe ermöglicht soziale Unterstützung. Hinzu kommt die Betreuung und Verstärkung durch die Gruppenleiter. Aus der Sicht der Forschung ist es im Vergleich zu Heimtrainingsprogrammen einfacher, die Menge der ausgeführten Bewegung zu erfassen.

Der Zwang, an bestimmten Tagen und Zeiten zu den bestimmten Einrichtungen zu fahren, kann die Sportteilnahme unbequem machen, was eine Barriere für die kontinuierliche Sportpartizipation sein kann. Zusätzlich entstehen durch die Mitgliedschaft in den Einrichtungen Kosten, die ein weiteres Hindernis darstellen.

Eine mögliche Alternative zu den beschriebenen klinik- und institutionsbezogenen Programmen bieten Bewegungsprogramme, die die Betroffenen nach Anleitung zu Hause allein durchführen können. Diese sind Kosten sparend. (Wing \& Jakicic, 2000; Wing, 2004).

Eine Reihe von Untersuchungen beschäftigen sich mit der Frage, ob supervisierte Bewegungsprogramme in Gruppen, die an ausgewiesenen Orten stattfinden, mehr oder weniger effektiv sind als ein Heimtraining oder „Lifestyle Physical Activity“Programm.

Fasst man die Ergebnisse der Studien zusammen, kommt man zu der Schlussfolgerung, dass beide Formen während der Anfangsphase einer Behandlung gut anwendbar sind. Heimtrainingsprogramme können langfristig gesehen einen Vorteil haben und bieten eine brauchbare Alternative $\mathrm{zu}$ in Gruppen angeleitetem Training. Dieses wiederum hat mehr Variationsmöglichkeiten, um die Teilnahme für 
passive Personen attraktiver zu gestalten (Wing \& Jakicic, 2000; Wing, 2004; Jakicic, 2003).

\subsubsection{Short Bouts}

Nach den aktuellen Richtlinien kann die empfohlene Menge an Aktivitäten auch durch die Akkumulation zeitlich kurzer Trainingseinheiten (short bouts) erreicht werden. Nach dieser „Theorie“ der „Short bouts“ werden kurze intermittierende Trainingseinheiten, die passend über den ganzen Tag verteilt werden, empfohlen (Franckowiak \& Andersen, 2003; Jackicic, 2003).

Diese besondere Sichtweise der Bewegung als Aufsummierung aller Aktivitäten wird aktuell im nordamerikanischen Raum erforscht. Ergebnisse vorläufiger Studien zeigen ähnliche kardiovaskuläre Wirkungen und Gewichtsverluste wie bei Teilnehmern traditioneller, durchgehender Bewegungsprogramme (Bensimhon et al., 2006; Blair \& Leermakers, 2004).

Andere Studien weisen darauf hin, dass das Verordnen der kurzen Trainingseinheiten zu einer verbesserten Teilnahme führen kann.

Eine weitere Studie mit kurzen intermittierenden Trainingseinheiten, in der zusätzlich Trainingsgeräte für das Heimtraining geliefert wurden, zeigte eine gute Aktivitätseinhaltung. Allerdings verbessert die Strategie der kurzen Trainingseinheiten nicht die langfristige Trainingsteilnahme, wenn man diese mit dem durchgehenden körperlichen Training vergleicht (Jakicic \& Otto, 2005).

Das Konzept der kurzen intermittierenden Trainingseinheiten kann für eine anfängliche Aneignung der körperlich-sportlichen Aktivität für Menschen mit Übergewicht und Adipositas sehr wirksam sein. Weiterhin kann es zu einer nachhaltigen Strategie für Menschen mit wenig Zeit werden. Sie ermöglicht Menschen körperlich aktiv zu bleiben, ohne eine zeitintensive Trainingseinheit in einem Fitnessraum oder zu Hause ausführen zu müssen (Franckowiak \& Andersen, 2003; Jakicic \& Otto, 2005).

Übergewichtige Personen, die von sich sagen, dass Trainingseinheiten von $30-45$ min überfordernd, langweilig oder schwierig in ihren Tagesablauf einzubeziehen sind, finden das Konzept der kurzen intermittierenden Trainingseinheiten häufig attraktiver.

Derartige kurze intermittierende Trainingseinheiten stellen eine weitere Alternative zur Teilnahme an Trainingsprogrammen dar, insbesondere in Zusammenhängen, in 
denen die Teilnahme an traditionellen, durchgehenden Trainingsprogrammen schwierig ist (Jakicic \& Otto, 2005).

\subsubsection{Die Individualisierung des Bewegungsprogramms}

Bei der Auswahl und Empfehlung einer sportlichen Aktivität oder eines Bewegungsprogramms werden zunächst Fördermaßnahmen, die sich mit standardisierten Maßnahmen an die gesamte Zielgruppe (one-size-fits-all intervention) wenden, kritisch diskutiert. Bewegungsprogramme, die auf die Bedürfnisse der Individuen zugeschnitten sind (tailored intervention), scheinen vielversprechend $\mathrm{zu}$ sein und werden dementsprechend empfohlen (Franckowiak \& Andersen, 2003).

Wenn ein reguläres Bewegungsprogramm auf die Bedürfnisse, Interessen und Fähigkeiten des Individuums zugeschnitten wird, statt als eine generelle Lösung „treib etwas Sport- get some exercise“ verwendet zu werden, dann wird es ein kraftvolles Instrument. Der Einzelne wird im Stande sein, den Sport auszuüben und so in seinen Bemühungen, Kontrolle über seine Gesundheit zu erlangen, unterstützt (Mannix et al., 1999).

Werden mögliche Barrieren bei der Erhaltung eines effektiven Bewegungsprogramms erkannt und ausgewiesen, ist ein Erfolg im Sinne einer Programmeinhaltung, die zu einer Verbesserung des Gesundheitsstatus führt, wahrscheinlicher (Mannix et al., 1999; Wadden \& Phelan, 2004).

Aus dem Grund werden bei der Verordnung und Empfehlung von Aktivitäten bestimmte Verfahren, wie die Bestimmung des aktuellen Gesundheitsstatus und die Erhebung der Sportbiographie, erforderlich. Es sollen folgende Aspekte berücksichtigt werden:

- die Interessen, Neigungen und die Bereitschaft des Patienten,

- die Akzeptanz und Freude an der jeweiligen Sportart,

- Energieverbrauch,

- Nebenwirkungen und Komplikationen (Wirth, 2003).

Vor dem Beginn eines Programms ist neben der obligatorischen ärztlichen Diagnostik eine motorische Basisdiagnostik (Ausdauerfähigkeit, Kraft, Körperzusammensetzung, Beweglichkeit) sinnvoll (Mannix et al., 1999). Eine Bestimmung des körperlichen Ausgangsleistungsniveaus ist eine wünschenswerte Ergänzung zum gegenwärtigen Gesundheitszustand bzw. ein Teilaspekt der Gesundheit. Die Erfas- 
sung des allgemeinen aktuellen Gesundheitszustandes und der körperlich-sportlichen Leistungsfähigkeit wird auf die offensichtlichen Patientenbedürfnisse wie Gewichtsreduktion, Blutdruckmanagement, Stressreduktion etc. hinweisen. Der Patient soll die Freiheit haben, eine angenehme Aktivität auszuwählen. Er soll selbst in der Lage sein, die Regelmäßigkeit seiner Aktivitäten zu variieren (Mannix et al., 1999).

Es ist wichtig, die individuellen Interessen der Patienten zu berücksichtigen. Wenn ein Programm verordnet wird, ohne die individuellen Wünsche erfragt zu haben, wird die Wahrscheinlichkeit der Compliance und dadurch des Erfolges verringert. Ein Mangel an persönlichem Einbeziehen des Patienten in die Auswahl verhindert die notwendige Identifikation mit dem Programm. Vorgeschlagene Aktivitäten sollen nicht als langweilig, schlimm oder gar als Strafe aufgenommen werden (Franckowiak \& Andersen, 2003).

Zusätzlich zu den Präferenzen des Patienten hinsichtlich der Sportarten soll auch erfragt werden, ob der Patient diese Aktivitäten allein oder in einer Gruppe betreiben möchte.

Die Erhebung der Sportanamnese und des aktuellen Bewegungsverhalten kann formell mit Hilfe von Fragebogen oder informell durch ein Gespräch durchgeführt werden (Wadden \& Phelan, 2004).

Durch diese Verfahren werden auch Einstellungen und Gefühle zur körperlichsportlichen Aktivität ausgedrückt. Durch die sportanamnestische Befragung können vergangene frustrierende und/oder schmerzhafte Erfahrungen erfasst werden. Sportbiographische Fragen sind bei vielen Adipösen mit negativen Gefühlen oder Enttäuschungen über vergangene Sportversuche verbunden. Das ist ein Umstand, der bei der Befragung in der Fragebogen- oder Gesprächsgestaltung berücksichtigt werden muss (Mannix et al., 1999; Franckowiak \& Andersen, 2003).

Die Informationen hinsichtlich des aktuellen Gesundheitszustandes, des Ausgangsniveaus der sportlichen Leistungsfähigkeit, der Bedürfnisse, Neigungen, Interessen und Sportgeschichte dienen dazu, das beste, individualisierte Bewegungsprogramm für die Interessenten verordnen zu können.

„Ein richtig dosiertes Bewegungsangebot kann dazu beitragen, Hemmschwellen abzubauen. Es wirkt motivierend und vermittelt Erfolgserlebnisse, und es stellt wieder einen positiven Bezug zum eigenen Körper her"(Vögele, 2003, S. 300). 


\subsubsection{Zielsetzung eines Bewegungsprogramms}

Der Erfolg eines gesundheitsfördernden Bewegungsprogramms bei Adipositas hängt in großem Maße von einer adäquaten Zielsetzung ab. Menschen mit Adipositas haben häufig hohe Erwartungen, und es werden unrealistische Ziele gesetzt, wie z.B. das Ziel einer raschen Gewichtsreduktion. Die Ziele sollten maßvoll gesteckt und auch kleine Erfolge verstärkt werden. Ein wichtiges Ziel kann z.B. die regelmäßige Teilnahme an einem Bewegungsprogramm sein, unabhängig davon, ob es zu einer Gewichtsreduktion kommt oder nicht. (Vögele, 2003). Die Erfolgswahrscheinlichkeit wird dadurch erhöht werden, dass die vereinbarten Ziele spezifisch, messbar und zeitabhängig sind (Mannix et al., 1999).

Der Therapeut hat die Aufgabe den Patient zu ermutigen, eine aktive Rolle bei der Mitgestaltung der eigenen langfristigen Ausgangsziele zu übernehmen. Die durch eine kurzfristige Partizipation erreichten Ziele sollen als Meilensteine des Fortschritts in Richtung endgültiger Zielsetzung dienen. Die Rolle des Therapeuten ist hier von großen Bedeutung: Er soll sicherstellen, dass die geeigneten Ziele gesetzt sind und dass der Patient versteht, wie diese Ziele erreicht werden können. Er soll in die Lage versetzt werden, diese realistische Zielsetzung fortzusetzen. Der Therapeut hat weiterhin die Aufgabe auf den Fortschritt und die erreichten Ziele aufmerksam zu machen, insbesondere wenn negative Gefühle auftauchen, wenn vorgenommene Ziele nicht erreicht worden sind. Hier kann es auch nötig werden, eine Zielsetzung zu korrigieren (Mannix et al., 1999; Franckowiak \& Andersen, 2003).

Unrealistischen Erwartungen an eine schnelle Gewichtsreduktion kann dadurch begegnet werden, dass den Patienten bewusst gemacht wird, dass es analog zu der Zeit, die es dauerte zuzunehmen, auch eine gewisse Zeit dauern wird, bis sie sicher und gesund ihr Übergewicht verlieren werden (Franckowiak \& Andersen, 2003).

Zusätzlich soll immer wieder auf die zahlreichen gesundheitsfördernden Konsequenzen der körperlichen Aktivität, die auch unabhängig von einer Gewichtsreduktion stattfinden, hingewiesen werden.

Es soll ein Bewegungsprogramm entwickelt werden, das sicher, gefahrlos, wirkungsvoll und angenehm ist (Mannix et al. 1999; Munsch \& Margraf, 2003). So kann verhindert werden, dass die Teilnehmer vorzeitig unmotiviert aufgeben (Woweries, 2004). 


\subsubsection{Die Bindung an das Bewegungsprogramm}

Damit ein Bewegungsprogramm hilfreich für die Erhaltung einer Gewichtsreduktion sein kann, ist es nötig, dass es über einen längeren Zeitraum eingehalten wird. Im Englischen spricht man von „adherence“, gemeint ist das „Dabeibleiben“, das „Weiterführen“ oder, wie Fuchs (2003) diesen Terminus übersetzt, die „Bindung“ an etwas.

Geht man der Frage nach, was die Personen, die Gewicht verloren haben und ihr neues Gewicht halten (maintainers), von denen, die Gewicht verloren haben, aber es wieder zugenommen haben (regainers), unterscheidet, so ist die kontinuierliche Aufrechterhaltung des Bewegungsprogramms entscheidend (Franckowiak \& Andersen, 2003; Wing \& Phelan, 2005). Die regelmäßige Teilnahme an einem Bewegungsprogramm ist ein zuverlässiger Prädiktor für eine langfristige Aufrechterhaltung der Gewichtsabnahme.

Obwohl die Rolle der Bewegung so wichtig für die Gewichtskontrolle ist, haben insbesondere Menschen mit Adipositas Schwierigkeiten ihre Trainingsteilnahme beizubehalten. Nach einem Literaturüberblick ist Adipositas an sich eine Barriere für die Teilnahme an körperlich-sportlichen Aktivitäten. Die Abbruchsraten bei Bewegungsprogrammen für adipöse Menschen liegen bei $70 \% \mathrm{im}$ Zeitraum zwischen dem 6. und 12. Monat. Andere prospektive Studien weisen darauf hin, dass $50 \%$ der Personen innerhalb der ersten 6 Monate eines Sportangebots ihre Teilnahme abbrechen werden (Wing \& Jakicic, 2000). Um die Aufrechterhaltung eines Bewegungsprogramms zu maximieren, ist es wichtig, die Gründe, die die Menschen für das Abbrechen ihres Programms angeben, in Betracht zu ziehen. Weiterhin können Strategien entwickelt werden, die Hindernisse beheben sollen. Die am häufigsten genannten Barrieren sind: Zeitmangel, Unbequemlichkeit, Mangel an Interesse (negative Einstellung und Gefühle gegenüber den Aktivitäten) und mangelnde soziale Unterstützung.

\subsubsection{Barrieren}

Im Folgenden werden Faktoren wie auch Verhaltensstrategien beschrieben, die diese spezifischen Barrieren auflösen und somit die Aufrechterhaltung eines Bewegungsprogramms fördern (Wing \& Jakicic, 2000; Franckowiak \& Andersen, 2003). 
- Barriere „Zeitmangel““

Zeitmangel ist ein häufig genannter Grund für das Scheitern einer regelmäßigen sportlichen Aktivität. Nach aktuellem Stand wird für einen adäquaten Energieverbrauch eine mittelintensive körperlich-sportliche Aktivität mit einer Dauer von 30-60 Minuten täglich benötigt (vgl. Kap. 4.8).

Will man den erforderlichen Level des Energieverbrauches in einer geringeren Zeit erreichen, wäre die Intensität der Trainingseinheit zu erhöhen. Studien, die die Effekte der Intensität eines Bewegungsprogramms auf die Aufrechterhaltung untersuchen, weisen auf keine einheitlichen Ergebnisse hin. Die Intensität eines körperlichen Trainings hat keinen deutlichen Effekt auf die Bindung an den Sport und verändert die Drop-out Raten nicht. Im Gegenteil, die sich zeigende erhöhte Abbruchsrate bei intensiven Trainingsprogrammen kann als ein Ergebnis der zugenommenen Häufigkeit der Sportverletzungen erklärt werden. Eine stufenweise Steigerung der Trainingsintensität kann die Verletzungsrate minimieren und zu einer besseren Aufrechterhaltung führen (Wing \& Jakicic, 2000).

Wichtig bleibt zu bedenken, dass der gesundheitliche Nutzen und die viele Vorteile für die Zielgruppe bei einem mittelintensiven Training am größten sind (Lombard \& Lombard, 2003).

Eine weitere alternative Vorgehensweise, um die Barriere „Zeitmangel“ zu überwinden, ist die Menschen zu ermutigen, zeitlich begrenzte kurze Trainingseinheiten, die sich im Laufe des Tages anhäufen, zu absolvieren. Diese Strategie der Akkumulation der Kurzen- Trainingseinheiten (Short- Bouts), die auch bei den „Lifestyle Activity“ Programmen Anwendung findet und durch die Empfehlungen der CDC und ACSM (1995) unterstützt wird, hat sich als gesundheitsfördernd erwiesen (Jakicic \& Otto, 2005).

Eine kontrollierte Studie von Jakicic, Wing, Butler \& Robertson (1995) konnte die positiven Ergebnisse der kurzen Trainingseinheiten hinsichtlich der Aufrechterhaltung des Sportprogramms zeigen. Im Rahmen der Untersuchung zeigte sich, dass die Gruppe der Teilnehmer mit den Kurztrainingseinheiten über einen längeren Zeitraum und in der Summe längere Zeit pro Woche als die Kontrollgruppe trainierte (Jakicic et al., 1995).

Dieses positive Ergebnis wird jedoch durch aktuellere Studien widerlegt (Jakicic \& Otto, 2005). 
- Barriere „Unbequemlichkeit““

Die Überzeugung vieler Personen, dass das sportliche Training unangenehm ist, stellt eine weitere Barriere der Sportteilnahme dar. Strategien, die die Annehmlichkeit des sportlichen Trainings steigern, können die Compliance verbessern. Sportliches Training wird angenehm, wenn es ein geselliges Erlebnis ist, auch lässt sich traditionelles Training gut durch moderne Elemente aus dem Wellnessbereich ergänzen.

- Barriere „Mangel an Interesse“

Ein anderes häufig zitiertes Hindernis ist der Mangel an Interesse. Diese Barriere versucht man zu überwinden, indem man versucht, flexiblere Möglichkeiten bei der Trainingsauswahl zu entwickeln, Stimuli im Sinne einer Umfeldkontrolle einzusetzen oder handfeste Belohnungen für das Training zu vergeben.

Mehr Flexibilität bei der Auswahl der Trainingsinhalte erhält man, indem die Teilnehmer ermutig werden, die Sportarten, an denen sie den meisten Spaß haben, auszuwählen und ihnen die Möglichkeit gibt, die Art des Trainings über die Zeit zu variieren (Wing \& Jakicic, 2000; Franckowiak \& Andersen, 2003; Woweries, 2004). Die oberste Prämisse bleibt, dass das Bewegungsprogramm Spaß macht und gesundheitlich unbedenklich ist.

Außerdem bekommen Patienten eine Erfahrung von Selbstwirksamkeit, wenn sie in die Gestaltung eines Trainingsprogramms miteinbezogen werden (Franckowiak \& Andersen, 2003).

Eine weitere Strategie gegen das mangelnde Interesse am körperlich-sportlichen Training ist die Anwendung von Techniken zur Stimuluskontrolle. Das Umfeld wird derart reorganisiert, dass es zur körperlichen Aktivität aufruft und antreibt.

Diese Aufforderungszeichen sollen spezifisch, d.h. in direkter Nähe zur zeitlichen und räumlichen Umgebung des Zielverhaltens, sein (Wing \& Jakicic, 2000; Lombard \& Lombard, 2003).

Mehrere Studien konnten die Effektivität einfacher Aufrufsignale und Antriebsstrategien demonstrieren. Charakteristisch ist die Studie von Brownell, Stunkard \& Albaum (1980), bei der die Forscher Hinweisschilder an bestimmten Stellen, an denen die Menschen zwischen Treppen oder Lift auswählen, plazieren. Diese einfache Intervention zeigte sich effektiv. Die Anzahl der Menschen, die Treppen nutzten, stieg signifikant. 
Telefonkontakte stellen eine weitere Antriebsform dar. Sie zeigen sich als effektives Mittel, Menschen zu ermutigen, ihr Training über einen längeren Zeitraum beizubehalten. Die Studie von King, Frey-Hewitt, Dreon \& Wood (1989) belegt die Effektivität dieser Vorgehensweise, auch wenn es sich zunächst um die beginnende Teilnahme an einem Bewegungsprogramm handelt (King, Frey-Hewitt, Dreon \& Wood, 1989, zitiert nach Wing \& Jakicic, 2000).

Eine Zusammenfassung der Literatur belegt die Effizienz der Telefoninterventionen zur Steigerung der körperlichen Aktivität. Dieses ist der Fall, wenn die Telefonkontakte als einziges Mittel einer Intervention oder in Kombination mit anderen Methoden des Patientenkontaktes angewendet werden (Jakicic, 2003). In einer Untersuchung von Lombard konnte belegt werden, dass nicht die Struktur und die Inhalte, sondern die Frequenz der Kontakte einen signifikanten Unterschied hinsichtlich der Steigerung der körperlichen Aktivität ausmachten (Lombard, Lombard \& Winett, 1995, zitiert nach Wing \& Jakicic, 2000).

Mehrere Studien untersuchten die Effektivität der Vermittlung von Komponenten behavioraler Interventionen zur Steigerung der körperlichen Aktivität via elektronischer Textnachrichten (E-mails). Materialien, die individualisiert und an die Bedürfnisse der Personen angepasst sind, erweisen sich als effektiver als gedruckte Standardmaterialien. Noch effektiver waren Materialien, die auf die aktuelle Motivationsphase der Person abgestimmt waren (Jakicic, 2003).

Eine Studie, in der Telefon- und E-mailkontakte während einer Follow-up-Periode von einem Jahr bei einer Gruppe von Patienten die Sport trieben und einer Gruppe von Patienten die Diät hielten angewandt wurden, zeigte, dass die Teilnehmer der körperlichen Trainingsgruppe im Vergleich zu den Teilnehmern der Diätgruppe im Laufe des „Follow-up-Jahres“ weniger Gewicht wieder zunahmen. Interessanterweise wurden keine Daten präsentiert, wie und ob das Trainingsverhalten durch die Kontakte beeinflusst wurde. (Wing \& Jakicic, 2000).

Einen Vergleich zwischen den beiden Antriebsinterventionen, Telefon- versus Emailkontakte, gibt es bisher nicht. Wenn die Effektivität dieser Strategien als ein Ergebnis einer sozialen Unterstützung entsteht, dann sollten die Telefonkontakte effektiver sein als der Kontakt via E-mails (Wing \& Jakicic, 2000).

Eine der am intensivsten untersuchten Strategien, um das Interesse an der körperlichen Aktivität zu steigern und die Bindung an die sportlichen Aktivitäten 
konsequent zu verbessern, ist die Anwendung konkreter Belohnungen, einschließlich Lotteriesystemen oder direkter Bezahlung für das körperlich-sportliche Training.

Die meisten Studien konnten die Effektivität indirekter Maßnahmen nicht bestätigen (Wing \& Jakicic, 2000). Indirekt bedeutet, dass die Teilnahme mit einem Los belohnt wurde, das dann vielleicht zu einem Gewinn führte. Mögliche Preise waren T-shirts, Schweißbänder oder Gutscheine eines Sportgeschäfts.

Direkte Belohnungen erwiesen sich als effektiv bei der Aufrechterhaltung eines sportlichen Trainings. Studien zeigten, dass das deponierte Geld am Beginn eines Kurses, welches am Ende des Kurses abhängig von Anwesenheit an den Kurssitzungen wieder zurückbezahlt wurde, die Sportpartizipation an diesem Kurs verbesserte (Wing \& Jakicic, 2000).

- Barriere „Sportstätten und Geräteausstattung“

Eine Strategie, die das positive Erleben von körperlichem Training erhöht, ist ein besserer Zugang $\mathrm{zu}$ den Sportanlagen und -einrichtungen. Daten einer Querschnittsstudie zeigen eine signifikante Korrelation zwischen der Nähe zur Sportstätte und dem Niveau der Selbstberichte über die körperliche Aktivität. Da es sich um Korrelationsberechnungen handelt, ist es schwierig, die Richtung der Korrelation zu bestimmen. Das heißt, entweder fördert das Wohnen in der Nähe von Sportstätten die sportliche Aktivität oder aktive Menschen wählen ihren Wohnsitz bewusst in der Nähe von Sportstätten (Sallis et al., 1990, zitiert nach Wing \& Jakicic, 2000).

Eine weitere Laborstudie konnte zeigen, dass, wenn der Zugang zu Sportgeräten erleichtert wird und der Zugang zu Geräten, die die Passivität fördern, erschwert wird, die Aussichten auf eine Aktivierung zunehmen. So kann die Ausgestaltung der Naherholungsgebiete wie Park, Fahrradwege, Freizeit- und Erholungscenter durch die Gemeinden und das Entfernen der Fernseher aus verschiedenen Räumen des Hauses eine effektive Vorgehensweise zu mehr körperlicher Aktivität auf einer groß angelegten Basis sein (Raynor, Coleman, \& Epstein, 1998, zitiert nach Wing \& Jakicic, 2000).

Eine weitere Möglichkeit, den Zugang zu ausreichender Bewegung zu erleichtern, ist die Option das Training im unmittelbaren Umfeld, dem Zuhause, durchzuführen.

Die US-Sportartikel-Industrie erfährt durch die gestiegene Auslieferung von Heimtrainern (Laufbänder, Stepper usw.) einen großen Zuwachs. Im Sportgeräte- 
Segment konnte der Verkauf 2004 mit 4,2 \% die größte Steigerungsrate auf fast 4,0 Mrd. \$ verbuchen. (Bundesagentur für Außenwirtschaft, 2005). Es ist dennoch wenig bekannt, ob durch den Zugang zu diesen Geräten auch wirklich die Raten der Trainingsteilnahme zunahmen.

Daten aus einer Querschnittsstudie von Jakicic, Wing, Butler \& Jeffery (1997) zeigen eine schwache, jedoch signifikante Beziehung zwischen dem Ausmaß des Heimtrainings auf den Geräten und dem gesamten Level der körperlichen Aktivität. Der Einsatz von Heimtrainern in einer weiteren Studie zeigte, wie der Zugang zu den Geräten zu einer besseren Einhaltung des Bewegungsprogramms führte. Die Kopplung eines verhaltenstherapeutischen Programms mit Heimtrainern kann eine effektive Strategie sein (Jakicic, Winters, Lang \& Wing, 1999).

Abschließend soll erwähnt werden, dass die Heimtrainergeräte auch die Funktion eines motivierenden Achtungssignals übernehmen können. Es besteht Bedarf nach weiterer Forschung auf diesem Gebiet, da sich keine umfassenden Schlussfolgerungen aus den wenigen Studien ziehen lassen (Wing \& Jakicic, 2000).

- Barriere „Mangel an sozialer Unterstützung“

Ein weiteres häufig zitiertes Hindernis für das sportliche Training ist die mangelnde soziale Unterstützung. Etliche Studien weisen auf einen Zusammenhang zwischen dem Ausmaß des sozialen Supports und dem Ausmaß des ausgeführten Trainings hin. Personen, die an einem Training mit ihrem Ehepartner teilnehmen, haben bessere Teilnahmeraten und niedrigere Abbruchsraten als verheiratete Personen, die allein teilnehmen (Wallace, Raglin \& Jastremski, 1995, zitiert nach Wing \& Jakicic, 2000).

Die Ergebnisse zweier Studien weisen darauf hin, dass der Versuch, die soziale Unterstützung zugunsten einer gesteigerten Bindung an die sportliche Aktivität zu nutzen, zu unterschiedlichen Ergebnissen führt.

Die Unterstützung durch den Übungsleiter bzw. Gruppenleiter über personenbezogene Rückmeldungen an die Teilnehmer verbesserte die Bindung an die Gruppe. $\mathrm{Zu}$ dieser Art der fortlaufenden Unterstützung für ein neues Verhalten zählen die erwähnten Aufforderungsstrategien über Telefon und E-mails (Wing \& Jakicic, 2000). 


\subsubsection{Rückfallprophylaxe und Bewältigungsstrategien}

Eine weitere Vorgehensweise zur Verbesserung der Bindung an die sportliche Aktivität ist die Rückfallprophylaxe und der Umgang mit Rückfällen. Mit Rückfall ist der dauerhafte Abbruch einer körperlichen Aktivität gemeint.

Es ist notwendig Strategien zu entwickeln, die kleine Trainingsversäumnisse nicht zu richtigen Rückfällen werden lassen. Dieser Ansatz, welcher auf der Theorie der Rückfallprävention von Marlatt und Gordon (1985) beruht, geht davon aus, dass alle Menschen von Zeit zu Zeit Trainingstermine nicht einhalten können, z.B. aufgrund einer Krankheit oder einer Verletzung. Die Lösung ist, den Teilnehmern zunächst beizubringen, diese als hohe Risikosituationen, die zu einem Rückfall führen können, zu erkennen. Des Weiteren werden den Teilnehmern Wege vermittelt, diese Situationen effektiv zu bewältigen. Den Teilnehmern wird beigebracht, wie sie nach einem Rückfall schnell zu ihrem regelmäßigen Training zurückkehren können (Marlatt \& Gordon 1985, zitiert nach Wing \& Jakicic, 2000).

Eine Studie an normalgewichtigen Frauen untersucht den Zusammenhang zwischen den Bewältigungsstrategien, den Risikosituationen und den Verhaltensmustern, denen ein Rückfall im Sinne eines Fernbleibens vom sportlichen Training folgt. Das Ergebnis der Studie unterstützt die These, dass die Vermittlung von Wissen an die Teilnehmer, wie sie mit Situationen hohen Risikos umgehen können, die Chancen der Rückfälle geringer werden lässt (Simkin \& Gross, 1994, zitiert nach Wing \& Jakicic, 2000).

Mehrere Untersuchungen, in denen der Schwerpunkt auf der Identifikation der Risikosituationen lag, konnten diesen Aspekt aufgrund methodischer Probleme nicht eindeutig belegen. Die Tatsache, dass das Rückfallprophylaxetraining ein Teil eines multifaktoralen Programms war, wie auch die kleinen Stichproben, machten eine Effizienzüberprüfung schwierig (Wing \& Jakicic, 2000).

\subsubsection{Verhaltenstherapeutische Strategien zur Unterstützung der Bindung}

Weitere Determinanten, die die Einführung und Bindung an ein Bewegungsprogramm für Erwachsene mit Adipositas beeinflussen können, sollen kurz erwähnt werden (Sallis \& Owen, 1999).

Manche dieser Variablen sind weitere Verfahren traditioneller verhaltenstherapeutischer Methoden (z.B. Selbstbeobachtung) und sie sind zum Teil eng mit dem 
oben Genannten verknüpft. Andere wiederum (z.B. Selbstwirksamkeit) haben eine zentrale Funktion in gesundheitspsychologischen Ansätzen.

- Eine flexible, z.B. von Woche zur Woche kurzfristig veränderbare, individualisierte, realistische und selbst bestimmte Zielsetzung kann für den Einstieg in ein Programm sehr nützlich sein (vgl. Kap. 4.9.7).

- Feedbackverfahren sind insbesondere effektiv, wenn sie mit anderen Verstärkungsstrategien kombiniert werden. Wenn das Feedback explizit und unmittelbar ist, ist auch die Bindung hoch. Individualisierte Rückmeldungen sind bei der Einführung eines Bewegungsprogramms effektiver als Gruppenrückmeldungen. Wenn Feedback mit einer spezifischen Zielsetzung angewendet wird, ist es für die Steigerung und Erhaltung des Trainings effektiv (Lombard \& Lombard, 2003).

- Die Anwendung einer Kosten-Nutzen-Analyse kann ein weiterer Aspekt bei der Steigerung eines sportlichen Trainings sein. Verschiedene Möglichkeiten wurden angewendet (z.B. Auflistung auf einem Papierblatt der Pros vs. Kontras einer möglichen Sportteilnahme), um die Kosten-Nutzen-Analyse einzuschätzen (Sallis \& Owen, 1999).

Die Ergebnisse der Untersuchungen sind dennoch uneinheitlich. Eine Erklärung für diese unterschiedlichen Ergebnisse liegt darin, dass eine am Beginn eines Programms erhobene Kosten- Nutzen-Analyse anders ausfallen kann als eine, die in der Mitte oder am Ende des Programms erhoben wird. Eine Lösung wäre, die Kosten-NutzenAnalyse zu verschiedenen Phasen des Programms durchzuführen und die verschiedenen Ergebnisse in ein Gesamtergebnis einfließen zu lassen.

Dadurch können die möglichen Kosten-Nutzen Veränderungen im Laufe eines Bewegungsprogramms aufgefangen werden. Es wird weiter so geplant, dass die positiven Verbesserungen (Nutzen) steigen und die Kosten (Aufwand) sich reduzieren (Lombard \& Lombard, 2003; Fuchs, 2003).

- Formen der Selbstbeobachtung werden allein und in Kombination mit anderen Verfahren angewendet. Diese Methode erwies sich als einfach und effektiv hinsichtlich einer Steigerung der körperlichen Aktivität, allerdings sind keine Effekte 
im Bezug auf das Beibehalten des Bewegungsprogramms notiert (Franckowiak \& Andersen, 2003; Lombard \& Lombard, 2003).

- Für eine erfolgreiche Aufnahme und regelmäßige Durchführung eines Bewegungsprogramms sind die Bereitschaft und weiterhin die Motivation eines Teilnehmers von großer Bedeutung. Es muss ermittelt werden, ob der ausgewählte Zeitpunkt, mit einem Bewegungsprogramm anzufangen, für den Betroffenen günstig ist (Timing). Die Bereitwilligkeit eines Patienten, sein Verhalten zu ändern, kann zunächst mit einer Steigerung der Alltagaktivitäten beginnen oder mit der Konsultation eines Verhaltenstherapeuten beginnen (Wadden \& Sarwer, 1999; Wadden \& Phelan, 2004; Franckowiak \& Andersen, 2003).

Bei der Frage nach der Motivation eines Teilnehmers wird zwischen einer extrinsischen und intrinsischen Motivation unterschieden. $\mathrm{Zu}$ Beginn der sportlichen Aktivitäten liegen bei den meisten Personen extrinsische Motive (wie z.B. das Gesundheitsmotiv) vor.

„Nur wenn ein Patient vom Nutzen des Sporttreibens überzeugt ist, nimmt er kurzoder mittelfristig die eher als unangenehm erlebte körperliche Aktivität in Kauf“ (Vögele, 2003, S. 301).

Die extrinsische Motivation, die sicherlich für die Aufnahme einer sportlichen Tätigkeit eine entscheidende Rolle spielt, kann für das Aufrechterhalten einer sportlichen Tätigkeit sogar hinderlich sein, da sie die intrinsische Motivation behindert. Die mit der extrinsischen Motivation verbundenen andauernden positiven Erwartungen an die Effekte der körperlichen Aktivität können von den unmittelbaren positiven Erlebnissen im Sporttreiben ablenken, so dass eine langfristige Bindung, die gerade durch das unmittelbare Erleben entsteht, unwahrscheinlicher wird.

Das Verhältnis von extrinsischer $\mathrm{zu}$ intrinsischer Motivation wird auch als ein natürlicher Übergang gesehen.

„Ein wichtiger Schritt von einer extrinsische zu einer intrinsischen Motivationslage besteht in der Fokussierung auf die körperliche Aktivität selbst und die Bewusstwerdung der Gefühle, die mit dieser Aktivität verbunden sind. Wenn der ausgeübte Sport genau den individuellen Fähigkeiten und Bedürfnissen entspricht, empfinden die betroffenen Personen, während sie sportlich aktiv sind, häufig ein hohes Maß an Zufriedenheit, Selbstkontrolle und Freude (Vögele, 2003, S. 299). 
Dieser Zustand wird in der englischsprachigen Literatur auch als "flow" bezeichnet und als zentraler Faktor intrinsischer Motivation betrachtet (Biddle \& Murtie, 2001). Ein Motivationsinterview kann hilfreich sein, um den Übergang von einer extrinsischen zu einer intrinsischen Motivation zu erleichtern. Ein solches Gespräch soll es dem Patienten erleichtern, seine eigenen Ziele bei der Ausübung von Sport besser zu erkennen und möglicherweise Modifikationen vorzunehmen. (Vögele, 2003; Wadden \& Phelan, 2004).

- Strategien, die die sportbezogenen Wirksamkeitserwartungen einer Person steigern, können auch die Sportpartizipationsraten beeinflussen. Unter sportspezifischer Selbstwirksamkeit ist die Überzeugung, die Kompetenz zu besitzen, ein sportliches Trainingsprogramm absolvieren zu können, zu verstehen (Fuchs \& Schwarzer, 1994). Die Selbstwirksamkeit im Bezug auf die Fortsetzung eines Bewegungsprogramms stellt ein wichtiges Problem für bestimmte Patienten dar. Eine langfristige Aufrechterhaltung der Teilnahme an sportlichen Aktivitäten kann höhere Levels an Verpflichtung (Commitment), Motivation und Überzeugung erfordern. Der Therapeut soll diese Themen berücksichtigen und den Patienten Verstärkung und Compliance bei seinen sportlichen und gewichtsreduzierenden Bemühungen anbieten (Franckowiak \& Andersen, 2003).

- Der Einsatz von Selbstkontrollstrategien wie auch kognitiven Techniken der Verhaltensmodifikation kann möglicherweise hilfreich sein. Unter Selbstkontrolle versteht man die Vermittlung von Techniken (Selbstverstärkung, Selbstbeobachtung, Selbstevaluation), die den Patienten helfen sollen, das eigene Verhalten selbst zu kontrollieren. Der Übergang von extern geleiteten Programmen zu Programmen in Eigenregie soll so erleichtert werden. Als kognitive Strategien werden das selbst organisierte Sporttreiben und das Training von Problemlösungsfertigkeiten zur Selbstkontrolle beschrieben (Fuchs, 2003; Lombard \& Lombard, 2003). Die Aneignung solcher Selbstkontrollstrategien ermöglicht es Personnen, Fertigkeiten zu entwickeln, die über Settings und Situationen hinaus angewendet werden können. Außerdem erwiesen sie sich als effektiv bzgl. der Aufrechterhaltung von Walkingprogrammen und „Lifestyle Activity“- Interventionen (Lombard \& Lombard, 2003). 
Zusammenfassend kann gesagt werden, dass eine Reihe der oben genannten Strategien vielversprechend hinsichtlich der Initiierung und der Beibehaltung von körperlichen Aktivitäten ist. Wenige dieser Verfahren sind speziell geeignet, die körperlichen Aktivitäten bei Menschen mit Adipositas $\mathrm{zu}$ steigern. Weitere Untersuchungen dieser Strategien sind nötig, um die genauen Wirkungen auf eine Zunahme der körperlichen Aktivität bei übergewichtigen Menschen zu evaluieren. Es ist unwahrscheinlich, dass jedes einzelne dieser Verfahren gleich effektiv auf alle Personen wirken wird, und es ist möglich, dass Trainingsversäumnisse und Rückfälle das Resultat von Zufällen sind, welche die Sportpartizipation beeinflussen. Das Wetter kann ein Hindernis für das Training in den Wintermonaten sein. Zeitmangel aufgrund familiärer Verpflichtungen oder mangelnde Motivation können Hindernisse sein, mit welchen eine Person unabhängig von der Jahreszeit konfrontiert wird. Deshalb sind unterschiedliche Strategien zu unterschiedlichen Zeiten nötig, und dies abhängig von den Barrieren, mit denen die Person aktuell beschäftigt ist.

Abschließend ist zu sagen, dass wenig darüber bekannt ist, welche Faktoren die Sportteilnahme nach einer Zeit von 6-12 Monaten beeinflussen und welche zum Erreichen und Halten der hohen Levels führen, die notwendig sind, um den Gewichtsverlust langfristig zu sichern (Lombard \& Lombard, 2003; Wing \& Jakicic, 2000). 



\section{Methodik}

Es handelt sich um ein Untersuchungsdesign im Zweigruppenvergleich. Verglichen werden eine sporttherapeutische Behandlungsform, in der psychoedukative Inhalte mit Bewegungselementen kombiniert werden, und eine Intervention, die ausschließlich aus Fitnesstraining besteht.

\subsection{Fragestellungen und Hypothesen der Untersuchung}

Bewegungstherapeutische Maßnahmen und Psychoedukation sind bei der Behandlung von Adipositas etablierte Inhalte der Behandlung. Im Rahmen der vorliegenden Arbeit wird die Effektivität bewegungstherapeutischer Maßnahmen mit und ohne psychoedukative Elemente im Rahmen eines ambulanten Programms bei Adipositas untersucht. Die Wirkungsweise der bewegungstherapeutischen Interventionen wird sowohl anhand psychischer als auch physischer Parameter überprüft.

Anhand des kontrollierten Zwei-Gruppenvergleichs werden folgende Hypothesen überprüft:

1. Bei den Teilnehmern(innen) des psychoedukativen Bewegungsprogramms und des Fitnessprogramms kann eine bedeutsame Gewichtsreduktion erreicht werden.

1.a Die Gewichtsreduktion in der Experimentalgruppe ist signifikant größer als in der Kontrollgruppe.

2. Die Körperzusammensetzung der Teilnehmern(innen) verändert sich während des ambulanten Bewegungsprogramms zu Gunsten der Muskelmasse.

2.a Der Anstieg der Muskelmasse ist in der Experimentalgruppe deutlicher als in der Kontrollgruppe.

2.b Der Anstieg der Fettmasse ist in der Experimentalgruppe deutlich geringer als in der Kontrollgruppe.

3. Die sportmotorischen Fähigkeiten, Ausdauer, Kraft und Koordination, verbessern sich im Rahmen des ambulanten Bewegungsprogramms. 
3.a Die Experimentalgruppe verbessert sich bezüglich der sportmotorischen Fähigkeiten und Fertigkeiten signifikant mehr als die Kontrollgruppe.

4. Die gesundheitsbezogene Lebensqualität verbessert sich im Rahmen des ambulanten Bewegungsprogramms.

4.a Die Experimentalgruppe verbessert sich bezüglich der gesundheitsbezogenen Lebensqualität mehr als die Kontrollgruppe.

5. Das Körperkonzept der Teilnehmer(innen) verändert sich während des ambulanten Bewegungsprogramms positiv.

5.a Die Verbesserung des Körperkonzepts zeigt sich in der Experimentalgruppe deutlicher als in der Kontrollgruppe.

6. Der Ausprägungsgrad der Depressivität nimmt bei den Teilnehmer(innen)n im Rahmen des Bewegungsprogramms bedeutsam ab.

6.a Hinsichtlich des Ausprägungsgrads der Depressivität zeigen sich in der Experimentalgruppe deutlichere Unterschiede als in der Kontrollgruppe.

7. Die Häufigkeit des pathologischen Essverhaltens nimmt in beiden Gruppen $\mathrm{ab}$.

7.a Hinsichtlich der Essstörungssymptomatik zeigen sich in der Experimentalgruppe deutlichere Unterschiede als in der Kontrollgruppe.

8. Die Teilnehmer(innen) beurteilen das ambulante Bewegungsprogramm positiv.

8.a Die Teilnehmer der Experimentalgruppe bewerten ihr Bewegungsprogramm positiver als die der Kontrollgruppe das reine Fitnesstraining.

9. Das Ausmaß an körperlichen und sportlichen Aktivitäten nimmt bei den Teilnehmer(inne)n während des Katamnesezeitraums deutlich zu.

9.a Der Anstieg bzgl. des Bewegungsverhaltens ist in der Experimentalgruppe deutlicher als in der Kontrollgruppe. 


\subsection{Die ambulanten Bewegungsprogramme als standardisierte achtwöchige Programme}

Sowohl das Bewegungsprogramm der Experimentalgruppe als auch das der Kontrollgruppe wurde in der Form eines standardisierten achtwöchigen Programms angeboten. Beide Programme umfassten insgesamt 20 Termine.

Die Experimentalgruppe nahm an dem psychoedukativen Bewegungsprogramm teil. Dieses bestand aus 8 Terminen Bewegungspsychotherapie, 8 Terminen mit funktions- und handlungsorientierten Inhalten (Wassergymnastik) in Kombination mit psychoedukativen Inhalten und 4 Terminen Walking. Der Wochenplan der Experimentalgruppe, welcher in dieser Struktur 3-mal wiederholt wurde, gestaltete sich wie folgt:

Zeit

Inhalt

Termine

\begin{tabular}{|l|l|l|}
\hline Montag 17:30 - 19:00 & Bewegungspsychotherapie & 8 \\
\hline Donnerstag $17: 30-18: 30$ & Wassergymnastik & 8 \\
$18: 30-19: 00$ & Edukation (Theorie) & \\
\hline Samstag /Sonntag & Walking & 4 \\
\hline
\end{tabular}

Abbildung 7: Wochenplan des psychoedukativen Bewegungsprogramms

Die Kontrollgruppe nahm an einem reinen Fitnesstraining teil. Alle 20 Termine (Fitness mit Musik, Gymnastik, Wassergymnastik und Walking) waren funktionsund handlungsorientiert.

Der Wochenplan einer Behandlungswoche der Kontrollgruppe, die in dieser Struktur 3-mal wiederholt wurde, gestaltete sich wie folgt:

Zeit
\begin{tabular}{|l|l|l|}
\hline Montag $17: 30-19: 00$ & Fitnesstraining & 8 \\
\hline Donnerstag $17: 30-18: 30$ & Wassergymnastik \\
$18: 30-19: 00$ & Freies Schwimmen & 8 \\
\hline Samstag /Sonntag & Walking & 4 \\
\hline
\end{tabular}

Abbildung 8: Wochenplan des Fitnesstrainingsprogramms 


\subsubsection{Methodik und Didaktik der Interventions- und Kontrollgruppe}

Für die Experimentalgruppe handelt es sich um ein körper- und bewegungsorientiertes Therapieprogramm, welches Fitness, Edukation, Motivationstraining und körpertherapeutische Elemente verbindet.

Die Methodik lässt sich allgemein als eine Kombination aus Vermittlung von Fertigkeiten und Wissen und dem Raumgeben für Bewegungs- und Körpererfahrung mit anschließender verbaler Reflektion beschreiben.

Konkreter lassen sich die Ziele und Inhalte in der Experimentalgruppe 3 Bausteinen zuordnen. Die der Kontrollgruppe entsprechen ausschließlich dem Baustein I (siehe Tabelle 10), wobei keine theoretischen Verbalinformationen über die eigentliche Übungsanleitung hinaus gegeben werden. Der zeitliche Umfang des Programms der Kontrollgruppe entspricht jedoch dem der Experimentalgruppe. 
Tabelle 10: Methoden und Inhalte von Experimental- und Kontrollgruppe

\begin{tabular}{|c|c|}
\hline Experimentalgruppe & Kontrollgruppe \\
\hline \multicolumn{2}{|l|}{ I. Therapiebaustein } \\
\hline $\begin{array}{l}\text { Inhalte sind Gymnastik, } \\
\text { Hockergymnastik, Spiele, } \\
\text { Wassergymnastik und Walking. }\end{array}$ & $\begin{array}{l}\text { Inhalte sind Gymnastik, } \\
\text { Hockergymnastik, Spiele, } \\
\text { Wassergymnastik und Walking. }\end{array}$ \\
\hline $\begin{array}{l}\text { Das Ziel ist der Aufbau der allgemeinen } \\
\text { Leistungsfähigkeit (Fitness), Erlangen } \\
\text { von Wissen und Kompetenzen zur } \\
\text { Realisation eines langfristigen gesunden } \\
\text { Bewegungsverhaltens. }\end{array}$ & $\begin{array}{l}\text { Das Ziel ist der Aufbau der allgemeinen } \\
\text { Leistungsfähigkeit (Fitness). }\end{array}$ \\
\hline $\begin{array}{l}\text { Die Gruppe erhält zusätzlich } \\
\text { differenzierte aufklärende verbale } \\
\text { Informationen. }\end{array}$ & $\begin{array}{l}\text { Es handelt sich um ein ausschließlich } \\
\text { übendes Verfahren }\end{array}$ \\
\hline \multicolumn{2}{|l|}{ II. Therapiebaustein } \\
\hline $\begin{array}{l}\text { Psychoedukative Einheiten dienen der } \\
\text { Ausarbeitung sportspezifischer Themen } \\
\text { und dem Führen eines } \\
\text { Bewegungstagebuchs. } \\
\text { Ziele sind: } \\
\text { - Kontrolle durch „Self Monitoring“, } \\
\text {-Vermittlung von Wissen, } \\
\text {-Therapie Compliance und Motivation, } \\
\text { - Sport Adherence nach Behandlung. } \\
\text { Methode: } \\
\text { Lernen durch Zuhören, Diskussion von } \\
\text { Arbeitsblättern, Angeleitetes Führen } \\
\text { eines Bewegungstagebuches durch } \\
\text { Gruppen- und Therapeutenfeedback. }\end{array}$ & \\
\hline \multicolumn{2}{|l|}{ III. Therapiebaustein } \\
\hline $\begin{array}{l}\text { Die themenzentrierte körperorientierte } \\
\text { Therapie beinhaltet } \\
\text { Körperwahrnehmungsübungen, } \\
\text { Bewegung mit Musik und } \\
\text { Entspannungsübungen. } \\
\text { Ziele sind die Verbesserung der } \\
\text { Körperakzeptanz, des Körper- } \\
\text { bewusstseins und des Selbstwertgefühls. }\end{array}$ & \\
\hline
\end{tabular}




\section{Zu Baustein I:}

Alle motorischen Fähigkeiten, Ausdauer, Kraft, Beweglichkeit sowie Koordination, wurden durch „Große und Kleine Spiele“, Aerobic und verschiedene gymnastische Bewegungsformen an Land und im Wasser trainiert. Die Verbesserung der Funktionsfähigkeit des Herz-Kreislaufsystems durch Ausdauerübungen (Aerobic, Walking) nahm einen hohen Stellenwert ein. Kraftübungen sollten muskuläre Dysbalancen abbauen und den Muskelaufbau fördern. Beweglichkeitsübungen (z.B. Stretching) sollten das Bewegungsausmaß verbessern bzw. erhalten und Fehlbelastungen verhindern. Koordinationsübungen zielten darauf ab, durch ökonomisch und richtig ausgeführte Bewegungen die Belastung des übergewichtigen Körpers gering zu halten.

\section{Zu Baustein II:}

a) - Einführung in das sport- und bewegungstherapeutische Programm: Ziele, Erwartungen

b) - die Rolle von Bewegung und Sport als Therapiemittel gegen Adipositas

c) - Umgang mit Risikoverhalten wie Überbeanspruchung und Sportverletzungen und deren Folgen

d) - Management von selbst organisierten sportlichen bzw. bewegungsreichen Aktivitäten

e) - Auswahl von Belastungsformen (Sportart), Dosierungen, d.h. Intensitäten, Häufigkeiten und Dauer unter der Berücksichtigung der Erkenntnisse aus der Trainingslehre und des Gesundheitssports

f) - Informationen zu Hygiene, Sport und Übergewicht

g) - Motivation unter Berücksichtigung des sozialen Umfeldes

h) - Planung sport- und bewegungstherapeutischer sowie freizeitorientierter Aktivitäten nach dem Sportprogramm

i) - Informationen zu Energieumsatz, Grundumsatz, Kalorienverbrennungen durch körperlich-sportliche Aktivitäten 


\section{Zu Baustein III:}

a) Körperkenntnis, Körperakzeptanz durch Körpererfahrungs- und Sensibilisierungsübungen (Schwerpunkte: Füße, Rücken, Schulter, Kopf, Bauch)

b) Übungen $\mathrm{zu}$ Regulation körperlicher Nähe und Distanz (Umgang mit Berührung)

c) Körpergrenzen, Abgrenzung, Ich- Stärkung

d) Umgang mit Gefühlen (z.B. Schamgefühl)

Jede Stunde ist so aufgebaut, dass sie neben dem vorgegebenen Schwerpunkt psychophysisches Aufwärmen und themenbezogene Entspannungsübungen als Ausklang mit beinhaltet.

Verbale Anteile ziehen sich zur Reflexion und Erfahrungsoptimierung durch alle Stundenteile.

\subsubsection{Zielsetzung des Treatments}

Die Stärkung kognitiver sportbezogene Gesundheitsressourcen im Sinne eines Ausbaus von Handlungskompetenz und Handlungswissen sind Hauptziele.

Hier liegt der Gedanke zugrunde, dass die Bindung an regelmäßige sportliche Aktivität durch die Vermittlung von Informationen erheblich gefördert werden kann. Wissen und Kenntnisse führen zu einer Steigerung der Handlungskompetenz und des Kontrollerlebens und damit zur Gewissheit, selbst etwas zur Gesundheit und Gesundheitserhaltung beitragen zu können.

Neben der pädagogischen Funktion bieten diese Therapiesequenzen die Möglichkeiten zu Diskussionen der Teilnehmer untereinander.

Die eingebrachten Themen werden direkt mit der eigenen Erfahrung und dem Erleben in Zusammenhang gebracht.

Konkrete Ziele auf der funktionellen und Beziehungsebene sind:

a)Physiologische Parameter

$>$ Gewicht (Reduktion)

$>$ Körperzusammensetzung (Fettanteil $\downarrow-$ Muskelmasse $\uparrow$ )

b)Motorische Parameter

$>$ Förderung von Ausdauer, Kraft, Koordination

$>$ Änderungen des Bewegungsverhaltens 
c)Psychologische Parameter

$>$ Verbesserung des Körperkonzepts

$>$ Entwicklung von Körperbewusstheit

$>$ Grenzen des Körpers erfahren

$>$ das ,Zuhause sein“ im eigenen Körper erfahren

$>$ Spannung und Entspannung erleben

$>$ psychophysische Zusammenhänge erleben

$>$ emotionalen Ausgleich über Bewegung erfahren

$>$ eigene Stärken und gesunde Anteile erleben

$>$ Freude an Spiel, Sport und Bewegung allein und mit anderen gewinnen

$>$ bisherige Erfahrungen korrigieren

$>$ realistische körperliche Selbsteinschätzung lernen

Konkrete Ziele auf der Metaebene sind:

$>$ Verbesserung der Einstellungen der Bewegung/dem Sport gegenüber

$>$ Verbesserung der Motivation bezüglich Bewegung und Sport

$>$ Probleme verbalisieren und reflektieren

$>$ Zusammenhänge von körperlichen Befindlichkeiten und psychischen Ereignissen verstehen

$>$ Wissen über den eigenen Körper erweitern

$>$ Erleben und Verstehen integrieren

\section{Nebengeordnete Zielsetzungen sind:}

$>$ Gruppenerfahrungen machen

$>$ Entscheidungen treffen

$>$ Frustrationstoleranz entwickeln

$>$ gegenseitiges Helfen erfahren

$>$ Ähnlichkeiten mit anderen erkennen

Körpersprache bei sich selbst und anderen beachten

$>$ Sich abgrenzen und sich öffnen können

Ausgewählte Stundenabläufe und Inhalte einzelner Stunden des Programms sind exemplarisch im Anhang dargestellt.

\subsection{Untersuchungsverfahren}

In der vorliegenden Untersuchung wird eine Reihe von subjektiven und objektiven Erhebungsinstrumenten eingesetzt. Diese werden in ihrer Konstruktion und in ihren Gütekriterien im folgenden Text beschrieben. 


\subsubsection{Fragebogen zu persönlichen Daten}

Durch diesen Fragebogen wurden soziodemographische Daten und der Modus, wie die Teilnehmer auf das Programm aufmerksam wurden, erfasst. Weitere Fragen zum allgemeinen Gesundheitszustand ermöglichten eine genauere Beschreibung der Probanden und dienten einer Eingangsdiagnostik bzgl. der Eignung zur Teilnahme am Bewegungsprogramm.

\subsubsection{Körpergewicht, Body Mass Index und Körperzusammensetzung}

Das Körpergewicht wurde mit der in das Bioimpedanzgerät (s.u.) integrierten Waage gemessen. Messzeitpunkt und Bedingungen (Kleidung, Tageszeit etc.) wurden vergleichbar gehalten.

Der Body Mass Index wurde nach dem ermittelten Gewicht und der angegebenen Körpergröße mit folgender Formel berechnet: Gewicht (kg) / (Körpergröße (m) $)^{2}$

Die Körperzusammensetzung wurde mit dem multifunktionalen Körperzusammensetzungsanalysegerät InBody 3.0 der Firma Biospace bestimmt.

Durch die Bioimpendanzanalyse kann der menschliche Körper in 4 Kompartimente eingeteilt werden (vgl. Kap. 2.1.1).

Die Ergebniswerte sind direkt aus den Messwerten (Impedanzen, Gewicht und Körpergröße) abgeleitet und unabhängig von den Eingaben „Alter“ und „Geschlecht“.

Die Messergebnisse auf dem Auswertungsbogen des InBody 3.0 zeigen das aktuelle Gewicht, die Körperzusammensetzung (Flüssigkeitshaushalt, Muskel-FettVerhältnis, Fettverteilung), Body Mass Index (BMI), Waist-to-hip-ratio (WHR) und legen einen Fitnesswert anhand der anthropometrischen Daten fest. Für die statistische Auswertung berücksichtigt wurden:

- das Körpergewicht,

- der BMI,

- die fettfreie Masse (FFM),

- Fettmasse. 


\subsubsection{Die Fahrradergometrische Belastungsuntersuchung}

Zur Bestimmung der körperlichen Leistungsfähigkeit wurden die Probanden einer fahrradergometrischen Belastungsuntersuchung in sitzender Position unterzogen, bei der mittels Polar Herzfrequenz-Messgerät (Modell PolarM2 $1^{\mathrm{TM}}$ ) die Pulsfrequenz abgenommen wurde. Die Fahrradergometrie lässt sich exakt dosieren und reproduzieren.

Für die Belastung wurde das WHO-Schema verwendet. Das Schema sieht eine Eingangsstufe von 25 Watt vor. Alle zwei Minuten wird die Belastung um 25 Watt stufenweise erhöht. Die Zahl der Umdrehungen ist für den Bereich zwischen 50 - 60 in der Minute festgelegt. Im Rahmen der vorliegenden Studie folgte der Belastungsphase eine dreiminütige Erholungsphase (90 Sekunden Ausfahren bei 25 Watt und 90 Sekunden Lockern der Beine). Die Funktionsparameter Herzfrequenz und arterieller Blutdruck wurden vor Beginn der Belastung, dann jeweils am Ende einer Belastungsstufe und schließlich in jeder Minute der Erholungsphase gemessen. Als Kriterien für einen vorzeitigen Abbruch der Belastung galten die von der Deutschen Gesellschaft für Kardiologie in den Leitlinien zur Ergometrie angegebenen Indikationen zum Abbruch der Belastung (Trappe \& Löllgen, 2000). Zur Beurteilung der Leistungsfähigkeit wurde die „physical working capacity“ (PWC) berechnet als Leistungsfähigkeit, die auf bestimmte Herzfrequenzen normiert wird (Rost, Heck \& Hollmann, o.J., 29). Für die Untersuchungen wurden die Frequenzmarken 130, 150 und 170 verwendet. Die pulsbezogene Leistungsfähigkeit wird in Relation zum Körpergewicht ausgedrückt und interpretiert. Für die Berechnung wurde das Körpergewicht, wie es in der Bioimpedanzbestimmung ermittelt wurde, herangezogen.

Die PWC-Werte ermöglichen über die intraindividuelle Veränderungsmessung hinaus eine interindividuelle Leistungseinschätzung anhand der geschlechtsspezifischen Normwerte (siehe Abbildung 9).

\begin{tabular}{|ccc|}
\hline & Männer & Frauen \\
& 1,5 & 1,25 \\
PWC $_{\mathbf{1 3 0}}$ & 2,0 & 1,6 \\
$\mathrm{PWC}_{\mathbf{1 5 0}}$ & 2,5 & 2,0 \\
$\mathrm{PWC}_{\mathbf{1 7 0}}$ &
\end{tabular}

Abbildung 9: Gewichtsbezogene Sollwerte der pulsbezogenen Leistungsfähigkeit für Männer und Frauen (nach Rost, Heck \& Hollmann, o.J., 30) 


\subsubsection{Fragebogen zum Gesundheitszustand (SF-36)}

Der Fragebogen zur gesundheitsbezogenen Lebensqualität, SF-36 Fragebogen zum Gesundheitszustand (Short-Form-36-Questionnaire), erfasst das subjektiv erlebte Befinden wie die physische und psychische Funktionsfähigkeit der Befragten. Der Fragebogen ist als Instrument zur Messung von Therapieerfolgen konzipiert und international anerkannt (Seidel \& Tijhuis, 2002).

Gesundheitsbezogene Lebensqualität als ein multidimensionales psychologisches Konstrukt ist hier durch vier Komponenten operationalisiert: das psychische Befinden, die körperliche Verfassung, die sozialen Beziehungen und die funktionale Kompetenz (Bullinger \& Kirchberger, 1998).

Der Fragebogen enthält acht Subskalen sowie die Bewertung der Gesundheitstendenz in insgesamt 36 Items (siehe Tabelle 11). Jedes Item thematisiert entweder selbst eine Skala bzw. ist Teil einer Skala.

Tabelle 11: Subskalen und Itemanzahl des SF-36-Fragebogens (Bullinger \& Kirchberger, 1998)

\begin{tabular}{|l|l|}
\hline Subskalen und Itemanzahl & Subskalen und Itemanzahl \\
\hline Körperliche Funktionsfähigkeit 10 & Vitalität 4 \\
\hline Körperliche Rollenfunktion 4 & Soziale Funktionsfähigkeit 2 \\
\hline Körperliche Schmerzen 2 & Emotionale Rollenfunktion 3 \\
\hline Allgemeine Gesundheitswahrnehmung 5 & Psychisches Wohlbefinden 5 \\
\hline
\end{tabular}

Die Antwortkategorien beim SF-36 variieren, es gibt Fragen, die einfach binär (ja nein) zu beantworten sind, bis hin zu sechsstufigen Antwortskalen.

Die Sensitivität der Skalen wurde auf die Messung der Veränderungen in Krankenpopulationen nach Ellert \& Bellach (1999) ,geeicht“.

Es handelt sich um ein Instrument, welches wegen seiner Kürze und seiner einfachen Handhabung für großen Erhebungsumfang in physischen und psychosozialen Gebieten empfohlen wird (Wadden \& Phelan, 2002).

Zusätzlich bietet das Instrument bevölkerungsrepräsentative Daten wie auch eine Normierung (z-Werte). Hier werden die erhobenen Lebensqualitätsdaten (Veränderungen des Punktwertes in einer Skala) mit den Veränderungen des 
klinischen Zustandes und Veränderungen des selbstberichteten Gesundheitszustandes in Relation zueinander gesetzt.

\subsubsection{Die Frankfurter Körperkonzeptskalen FKKS nach Deusinger (1998)}

Die FKKS sind ein Fragebogenverfahren, das sich aus neun eindimensionalen Skalen zusammensetzt. Sie dienen „zur Bestimmung des jeweiligen Bildes oder Selbstkonzepts, das das Individuum in dem wichtigen auf den Körper bezogenen Bereich des Selbst von der eigenen Person entwickelt hat" (Deusinger, 1998, S. 8). In Tabelle 11 sind die Skalen mit den dazugehörigen Items aufgeführt. Der Fragebogen umfasst insgesamt 64 Items, für die es jeweils sechs Antwortmöglichkeiten gibt. Mit Hilfe einer Auswertungsschablone wird für jedes Item die Punktzahl von eins bis sechs bestimmt und das Item einer der 9 Subskalen zugeordnet. Der Skalenwert wird durch Addition der jeweiligen Itempunkte ermittelt. Er entspricht einem negativen, neutralen oder positiven Körperkonzept in der jeweiligen Skala. Der Wert des Gesamt-Körperkonzepts errechnet sich aus der Summe aller neun Skalenwerte.

Tabelle 12: Die neun Skalen der Frankfurter Körperkonzeptskalen, die Kürzel und die zugehörigen Items (Deusinger, 1998)

\begin{tabular}{|c|c|c|}
\hline Skala & Bereich & Item \\
\hline SGKB & Gesundheit, körperliches Befinden & $10,15,25,40,44,45$ \\
\hline SPKF & $\begin{array}{l}\text { Pflege des Äußeren und } \\
\text { körperlichen Funktionsfähigkeit }\end{array}$ & $11,17,28,53,56,58,60,61$ \\
\hline SKEF & Körperliche Effizienz & $13,16,24,29,30,51,52,57,62,63$ \\
\hline SKKO & Körperkontakt & $8,12,19,26,39,50$ \\
\hline SSEX & Sexualität & $4,6,31,34,48,54$ \\
\hline SSAK & Selbstakzeptanz des Körpers & $1,2,5,7,18,33$ \\
\hline SAKA & Akzeptanz des Körpers durch andere & $3,21,41,49$ \\
\hline SASE & Aspekte der körperlichen Erscheinung & $\begin{array}{l}9,14,20,22,23,27,35-38,42,46, \\
47,64\end{array}$ \\
\hline SDIS & Dissimilatorische Prozesse & $32,43,55,59$ \\
\hline
\end{tabular}


Die FKKS sind in der Durchführung und Auswertung ein ökonomisches und hoch objektives Verfahren. Es ist das einzige Verfahren, welches das Körperkonzept anhand dieser Vielfalt von Faktoren (Subskalen) erfasst.

Die innere Konsistenz (Cronbachs Alpha) der Gesamtform liegt bei $\mathrm{r}=0,88$, die einzelnen Skalen zwischen 0,62 und 0,93. Test-Retest-Reliabilität des Gesamtwerts liegt zwischen 0,62 und 0,93, die der einzelnen Skalen zwischen 0,28 und 0,97 auf der Grundlage von Messungen über Zeiträume von vier Tagen bis zu drei Monaten. Im Manual sind zahlreiche Studien zur Validität enthalten, sowohl mit Patienten unterschiedlicher Krankheitsbilder als auch über den Vergleich mit anderen Persönlichkeitsinventaren. Ebenso liegen im Manual Mittelwerte und Prozentränge nach Alter und Geschlecht getrennt sowie Normwerte für Patienten mit Essstörungen vor.

\subsubsection{Beck-Depressions-Inventar (BDI)}

Das Beck-Depressions-Inventar (BDI) nach Hautzinger, Bailer, Worall und Keller (1995) ist ein gut validiertes Selbstbeurteilungsverfahren. Es dient der Bestimmung des Schweregrades der Depressivität, zur Messung von Veränderungen während der Behandlung und von Schwankungen der Befindlichkeit und depressiver Beschwerden.

Der Fragebogen enthält 21 Gruppen von Aussagen/Items. Jedes Item wird auf einer vierstufigen Skala beantwortet in Hinblick auf das Auftreten und die Intensität während der vergangenen sieben Tage.

Die Auswertung erfolgt durch die Addition der angekreuzten Aussagen, wobei für jede Gruppe von Aussagen nur die am höchsten zählende Aussage berücksichtigt wird.

Bei der Anwendung des BDI in der Studie ergab sich Zweifel an der Validität eines Items. Das 19. Item mit dem Buchstaben $\mathrm{S}$ zur Gewichtsreduktion lässt sich sehr kritisch in der gesamten Stichprobe verwenden, da nach Bewegungsprogrammen höchstwahrscheinlich keine unbeabsichtigte Gewichtsabnahme vorkommt. Eine mögliche Gewichtsabnahme sollte hier nicht als ein depressives Symptom aufgefasst werden. Eine Gewichtsabnahme bei den Teilnehmern ist ein Ziel des Treatments. Sie kann zusätzlich auch durch Diät oder andere Verhaltensänderungen erreicht werden. 
In der vorliegenden Untersuchung wurde das 19. Item S, das sich auf eine Gewichtsabnahme als eine depressive Symptomatik bezieht, dennoch in der Auswertung berücksichtigt und der Score des Items wird in den Summenwert addiert. Aus dem Grund könnten die BDI- Summenwerte das tatsächliche Ausmaß der Depressivität leicht überschätzen.

Wegen der geschilderten Einschränkung können die Vergleiche mit der Norm sowie die Darstellungen der Veränderungen gemäß der im BDI-Handbuch (Hautzinger et al., 1995) vorgeschlagenen Kategorien nur entsprechend vorsichtig interpretiert werden.

Anhand des Summenwerts können die Patienten bzgl. ihrer Depressivität 3 Klassen zugeordnet werden.

Tabelle 13: Einteilung der depressiven Symptomatik nach Summenwert im BDI (nach Hautzinger et al., 1995)

\begin{tabular}{|l|l|}
\hline Summenwert & Ausprägung der depressiven Symptomatik \\
\hline$<11$ & unauffällig, gesund \\
$11-17$ & milde bis mäßig \\
$\geq 18$ & klinisch relevant \\
\hline
\end{tabular}

Die Werte der inneren Konsistenz (Cronbachs Alpha) liegen in Abhängigkeit von der Stichprobe zwischen $r=.73$ und $r=.95$. Die Stabilität der Symptomatik liegt über eine Woche bei $r=.75$ und über zwei Wochen bei $r=.68$.

Die inhaltliche Validität ist auch nach den neuen Diagnosekriterien gegeben. Mit Ausnahme eines Kriteriumssymptoms werden alle in den gegenwärtig gültigen psychiatrischen Diagnosesystemen als relevant betrachteten Beschwerden abgebildet. Korrelationen mit anderen Selbstbeurteilungsskalen bei depressiver Symptomatik liegen bei $r=.76$ (Hautzinger et al., 1995).

Der Fragebogen wird wegen seiner weit verbreiteten Anwendbarkeit empfohlen. Der BDI ist für den Bereich der Adipositasforschung gut geeignet und gilt als sehr aufschlussreich bei Gewichtsreduktionsinterventionen und verhaltenstherapeutischen Interventionen (Wadden \& Phelan, 2002). 


\subsubsection{Das strukturierte Inventar für anorektische und bulimische Essstörungen} (SIAB)

Das strukturierte Inventar für anorektische und bulimische Essstörungen (SIAB) von Fichter und Quadflieg (1999) liegt als Experteninterview und als Fragebogen zur Selbsteinschätzung vor. Dieser enthält jeweils 87 Fragen zu Symptomen von Essstörungen und weiteren Störungen (Depression, Angst), sozialer Integration, Sexualität, Ausschlussdiagnosen und Medikation. Weitere Fragen, die zur Erstellung von Diagnosen erforderlich sind, sind enthalten. Folgende Diagnosen können mit dem in der vorliegenden Untersuchung verwandten SIAB-EX erstellt werden:

Anorexia nervosa nach DSM IV

- Bulimia nervosa nach DSM IV

- Nicht näher bezeichnete Essstörung nach DSM IV einschließlich Binge Eating Disorder nach den im Anhang des DSM-IV beschriebenen Kriterien Anorexia nervosa nach ICD-10

Atypische Anorexia nervosa nach ICD-10

- Bulimia nervosa nach ICD-10

- Verschiedene zusätzliche Essstörungs-Syndrome

Der SIAB ist einsetzbar bei Jugendlichen und Erwachsenen im Alter von 12 bis 65 Jahren. Der Fragebogen setzt sich aus 6 Subskalen und einer Gesamtskala zusammen. Die Subskalen erfassen essstörungsspezifische (z.B. Essanfälle, Gewichtsphobie) und weitere Psychopathologie (z.B. Depression, Angst) sowie soziale Integration und Sexualität.

Die Daten werden durch geschulte Experteninterviewer erhoben (SIAB-EX). Die Durchführungsdauer beträgt 30 - 60 Minuten.

Die Auswertung ergibt sich durch Berechnung der Subskalen und Erstellung der Diagnosen nach vorgegebenen Anweisungen. Als Hilfsmittel liegen Algorithmen und Formblätter zur Handauswertung vor.

Es liegen Vergleichswerte zu 377 wegen Essstörungen behandelten Männern und Frauen und auch zu gesunden Frauen vor. Das Datenmaterial ist auch nach den Diagnosen Anorexia nervosa und Bulimia nervosa nach DSM-IV getrennt vorhanden (Fichter \& Quadflieg, 1999).

Die Durchführungs-, Auswertungs- und Interpretationsobjektivität ist gewährleistet. Befunde zur konvergenten und diskriminanten Konstruktvalidität liegen vor. Es 
liegen hinreichende Befunde zur Beobachterübereinstimmung (SIAB-EX), internen Konsistenz und Übereinstimmung von SIAB-EX und SIAB-S vor. In der vorliegenden Studie wurden die für eine Diagnose nach DSM IV relevanten Fragen gestellt. Diese für die Diagnostik relevanten Fragen beinhalten im Titel eine Kodierung.

\subsubsection{Der Dortmunder Fragebogen zur Bewegungstherapie}

Der Dortmunder Fragebogen zur Bewegungstherapie (DFBT) von Hölter wurde in Anlehnung an ein Konzept von Yalom (1989) entwickelt und erfasst verschiedene Wirkfaktoren der Bewegungstherapie.

Nach den theoretischen Vorlagen ging Hölter zunächst von 7 Subskalen aus. Erste statistische Analysen zur Struktur des Fragebogens mit Hilfe von Faktorenanalyse brachten keine befriedigenden Ergebnisse (Hölter, 2004).

Eine neuere Veröffentlichung von Ungerer-Röhrich (2001) schlägt eine 4Faktorenlösung vor. Die Überprüfung der Faktorenstruktur wurde anhand einer Patientenstichprobe von $\mathrm{N}=240$ vorgenommen.

Der Fragebogen in der neueren Form besteht aus 22 Items, die auf einer fünfstufigen Skala von „trifft sehr zu“ bis „trifft überhaupt gar nicht zu“ beantwortet werden.

4 Subskalen erfassen die Bereiche: biographische Erfahrungen, Erfahrungen von Körper und Selbst, Erleben von Beziehungen und Erleben von Bewegung und Wohlbefinden. Die vorliegende Arbeit benutzt die neuere Version, die sich auch in anderen aktuellen bewegungstherapeutischen Untersuchungen durchsetzt (Hölter, 2004).

\subsubsection{Fragebogen zur sportlichen und körperlichen Aktivität}

Der Fragebogen zur Erfassung der sportlichen und körperlichen Aktivität soll Auskunft über die aktuellen sportlichen und körperlichen Aktivitäten der Teilnehmer in Freizeit und Beruf geben.

Bei der Erfassung der körperlichen Aktivität werden drei Bereiche unterschieden:

- Sportliche Aktivität

- Körperliche Aktivität in der Freizeit (außer beim Sport)

- Körperliche Aktivität im Beruf 
Darüber hinaus wird in der vorliegenden Studie zwischen aktueller und habitueller sportlicher Aktivität unterschieden. Die aktuelle sportliche Aktivität wird aufgrund der Angaben zur Intensität, Umfang und Dauer der Aktivitäten errechnet. Die habituelle sportliche Aktivität wird durch retrospektive Angaben zur sportlichen Aktivität im Vergleich zu aktuellen sportlichen Aktivitäts-Typen gebildet.

Die körperliche Aktivität in der Freizeit - außerhalb der sportlichen Aktivität - wird durch die Befragung nach den täglichen Geh- und Radstrecken sowie nach der Ausübung von körperlich anstrengenden Freizeittätigkeiten ermittelt.

Für die Erfassung der körperlichen Aktivität im Beruf wurde sowohl nach der Art der überwiegenden Beanspruchung (sitzend, stehend, in Bewegung) als auch nach dem Ausmaß der Belastung gefragt.

Der Fragebogen ist für die Erfordernisse der Studie zusammengestellt. Er besteht aus 15 Items und ist eine Modifikation des Fragebogens von Woll (2004), der in der Studie „Kommunale Gesundheitsförderung in Bad Schönborn“ angewendet wurde.

\subsubsection{Der Walkingtest}

Der Walkingtest ist ein Testverfahren zur Erfassung der körperlichen Ausdauerleistungsfähigkeit. Es wurde für die Zielgruppe der älteren und leistungsschwächeren Menschen, wie z.B. Übergewichtige, entwickelt, für die die üblichen Lauftests ungeeignet sind.

Nach den Testerfindern eignet sich der Walkingtest besonders für übergewichtige gesunde Personen ohne gesundheitliche Risikofaktoren, wie kardiovaskuläre Erkrankungen (Bös, 1994).

Für die Durchführung des Walkingtests legen die Testteilnehmer eine Strecke von 2 km im zügigen gleichmäßigen Gehen auf einer ebenen Wegfläche (z.B. Laufbahn) zurück.

Es handelt sich um einen aussagekräftigen, einfachen und praktikablen Test.

Für die Auswertung werden folgende Messgrößen erfasst:

- Gehzeit für die 2 km-Strecke in Minuten und Sekunden

- Belastungspuls (Schläge/min) nach $2 \mathrm{~km}$

- Relatives Körpergewicht (BMI)

- Lebensalter 
Durch eine Berechnungsformel wird ein Leistungsindex ermittelt, der die Interpretation der Testergebnisse durch eine Zuordnung $\mathrm{zu}$ einer von 5 Leistungskategorien von „sehr gut“ bis „sehr schwach“ ermöglicht. Die genaue Einteilung der 5 Stufen findet sich in Kap. 6.8 und wird dort in Zusammenhang mit den Ergebnissen des Walkingtests dargestellt.

Der Index wird nach der folgenden Formel berechnet (Bös et al., 1992):

\begin{tabular}{|c|c|}
\hline $\begin{array}{lll}\text { Berechnung } & \text { des } & \text { Walking- } \\
\text { Leistungsindex für Frauen } & \end{array}$ & 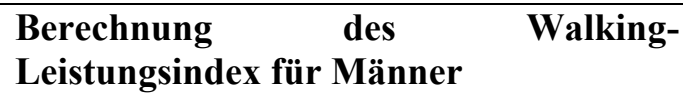 \\
\hline $\begin{array}{l}\text { Walkingzeit }(\min )-\text { x } 8,1= \\
\text { Walkingzeit (sek) }- \text { x } 0,14=- \\
\text { Herzfrequenz — } 0,36=- \\
\text { BMI }(\mathrm{kg} / \mathrm{m} 2) \_ \text {× } 1,0=-\end{array}$ & 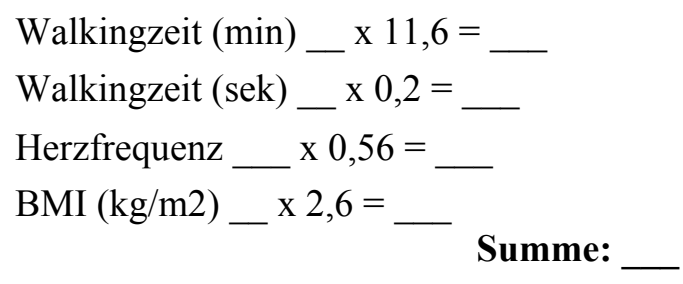 \\
\hline subtrahieren von der Summe & subtrahieren von der Summe \\
\hline Alter (Jahre) _ x 0,3= & Alter (Jahre) _ x 0,2= \\
\hline Subtraktion: & Subtraktion: \\
\hline subtrahieren von dem Koeffizienten 305 & subtrahieren von dem Koeffizienten 420 \\
\hline Walking-Index: & Walking-Index: \\
\hline
\end{tabular}

Abbildung 10: Berechnungsformel des Walking-Index für Frauen und Männer nach (Bös et al., 1992)

Korrelationskoeffizienten von 0,75 bis 0,97 auf der Basis regressionsanalytischer Untersuchungen bei Studien mit Normalgewichtigen sprechen für die Validität des Walkingtests (Bös et al., 1992).

Bei einer Validitätsstudie mit übergewichtigen gesunden Testpersonen lagen die Zusammenhänge jedoch etwas niedriger. Der Korrelationskoeffizient zwischen direkter und indirekter $\mathrm{V}_{2}$-max- Messung war bei den Männern 0,75 bei den Frauen 0,77 .

So liefert der Walkingtest auch bei übergewichtigen Testpersonen valide und reliable Ergebnisse der $\mathrm{V0}_{2}$-max- Schätzung (Bös, 1994). 


\subsubsection{Der Bewegungskoordinationstest für die Praxis der Therapiekontrolle BKT -Kur nach Bös, Wydra und Karisch (1992)}

Der BKT-Kur wurde als Test zur Erfassung der Bewegungskoordination von Erwachsenen konzipiert und lässt sich im Rahmen einer Therapiekontrolle einsetzen. Er besteht aus zehn Items, die dichotom bewertet werden (Aufgabe gelöst bzw. nicht gelöst). Gelöste Aufgaben werden mit je einem Punkt, ungelöste mit keinem Punkt gewertet. Das Gesamtergebnis wird durch Addition aller Punkte berechnet und stellt ein $\mathrm{Ma}$ für die koordinative Leistungsfähigkeit dar.

Die ursprüngliche Form des Bewegungskoordinationstests ist in mehreren Studien evaluiert worden. Die endgültige, in dieser Untersuchung eingesetzte Version wurde auf den Untersuchungen von 1800 Testpersonen basierend entwickelt.

Der Test ist ökonomisch in Kleingruppen durchzuführen und einfach auszuwerten.

Das Verfahren ist hoch objektiv, der Korrelationskoeffizient liegt bei $\mathrm{r}=.97$ zwischen zwei Testbeobachtern.

Die Reliabilität wird für die innere Konsistenz (Cronbachs Alpha) mit $r=.72$ angegeben. Im Test-Retest-Verfahren liegt die Reliabilität bei $r=.60$ (Bös et al., 1992).

Die Validität wurde anhand von mehreren Untersuchungen herausgestellt. Die Testergebnisse können mit den alters- und geschlechtsabhängigen Normwerten verglichen werden (siehe Tabelle 14).

Tabelle 14: Normwerte für den Bewegungskoordinationstest $(N=1831)$ nach Bös et al., (1992) in Abhängigkeit von Geschlecht und Alter

\begin{tabular}{|c|c|c|c|c|c|}
\hline \multicolumn{2}{|c|}{$\begin{array}{l}\text { Summenscore beim } \\
\text { BKT-Kur }\end{array}$} & \multicolumn{4}{|c|}{ Altersgruppen in Jahren } \\
\hline sehr gut & Männer & $\begin{array}{c}20-29 \\
>8\end{array}$ & $\begin{array}{c}30-39 \\
>8\end{array}$ & $\begin{array}{c}40-49 \\
>8\end{array}$ & $\begin{array}{c}50-59 \\
>7\end{array}$ \\
\hline & Frauen & $>8$ & $>8$ & $>7$ & $>6$ \\
\hline \multirow[t]{2}{*}{ gut } & Männer & 8 & 8 & 8 & 7 \\
\hline & Frauen & 8 & 8 & 7 & 6 \\
\hline \multirow[t]{2}{*}{ mittel } & Männer & $6-7$ & $6-7$ & $6-7$ & $5-6$ \\
\hline & Frauen & $6-7$ & $6-7$ & $5-6$ & $4-5$ \\
\hline \multirow[t]{2}{*}{ schwach } & Männer & 5 & 5 & 5 & 4 \\
\hline & Frauen & 5 & 5 & 4 & 3 \\
\hline \multirow[t]{2}{*}{ sehr schwach } & Männer & $>5$ & $>5$ & $>5$ & $>4$ \\
\hline & Frauen & $>5$ & $>5$ & $>4$ & $>3$ \\
\hline
\end{tabular}




\subsubsection{Der Krafttest}

Der Krafttest ist ein Verfahren zur Erfassung der Kraftausdauer der Beine, des Rumpfes und der Arm- Schultermuskulatur. Er besteht aus 3 Items, „Jump and reach“ „Sit- ups“ und „Liegestütz“ Die Items entstammen der Motorischen Basisdiagnostik (MBD) nach Bös, Wydra und Karisch (1992) und wurden in der vorliegenden Untersuchung teilweise für die Zielgruppe der Übergewichtigen modifiziert.

Die Beschreibung der einzelnen Items sowie die Bewertungskriterien befinden sich im Anhang.

Die Objektivität und Reliabilität aller 3 Items liegt bei 0,70 - 0,90. Die inhaltliche Validität der Testaufgabe „Jump and reach“ korreliert mit der Maximalkraft der Beinstrecker mit $r=0,92$. Zu den Items „Liegestütz“ und „Sit ups“ machen die Testentwickler keine numerischen Angaben.

Für alle 3 Testaufgaben liegen Normwerte von Gesundheitssportlern und Rehabilitanden vor.

\subsection{Untersuchungsstichprobe}

Bei den Probanden handelt es sich um Männer und Frauen mit Adipositas zweiten Grades $(\mathrm{BMI} \geq 35)$. Die Teilnehmer rekrutieren sich aus der Region Chiemgau, den Landkreisen Rosenheim und Traunstein.

Ausschlusskriterien waren:

- Körperliche Grund- oder Folgeerkrankungen, die eine körperliche Belastung ausschließen

- Teilnehmer mit einem BMI $<35$

- Gravidität (aus Gründen der wissenschaftlichen Vergleichbarkeit ${ }^{2}$ )

- Teilnehmer, die weniger als 8 Wochen im Programm bleiben konnten

Die Bekanntmachung des ambulanten Programms in der Bevölkerung und somit die Rekrutierung der Probanden erfolgte auf mehreren Wege. Annoncen in der lokalen

${ }^{2}$ Die Behandlungsart eignet sich bei normalem Verlauf der Schwangerschaft auch für Schwangere. 
Zeitung, Verteilung von Flyern über die Krankenkassen, Volkshochschulen und Apotheken sowie das Anschreiben niedergelassener Ärzte waren Informationswege. Als besonders effektiv erwies sich jedoch die Bekanntmachung des Programms durch die mündliche Mitteilung der Teilnehmer an andere Interessenten in ihrem sozialen Umfeld.

Die Interessenten meldeten sich postalisch oder telefonisch. Sie wurden dann vom Studienleiter zurückgerufen und über das Vorhaben informiert. Darüber hinaus wurden erste persönliche Termine vereinbart.

Wenn die Interessenten die Teilnahmebedingungen erfüllten, wurden sie ins Bewegungsprogramm aufgenommen. Bei Teilnahme unterschrieb jeder Teilnehmer eine Einwilligungserklärung.

Als Anreiz zur Partizipation galten für alle Teilnehmer das körperliche Training, zusätzliche Informationen über ihre Körperzusammensetzung und ihre Leistungsfähigkeit sowie eine Verlaufskontrolle.

Der Versuchsgruppe wurde darüber hinaus das psychoedukative Bewegungsprogramm geboten.

Die Einteilung in Versuchs- oder Kontrollgruppe ergab sich zufällig über die Reihenfolge der Anmeldungen.

\subsection{Methodische Überlegungen zu dem Untersuchungsdesign}

Für die Evaluation des ambulanten psychoedukativen Bewegungsprogramms wurde das Design eines Zweigruppenvergleichs ausgewählt. Die Wirkung des psychoedukativen Bewegungsprogramms wurde in zweifacher Hinsicht versucht zu klären. Das psychoedukative Bewegungsprogramm wurde mit dem Fitnesstrainingsprogramm verglichen und es wurden Eigenwartegruppen mit einer Wartezeit von 4 Wochen bis zum Behandlungsbeginn geschaffen. 


\subsubsection{Untersuchungsdesign}

Das Untersuchungsdesign lässt sich als kontrollierter Zwei-Gruppen-Versuchsplan graphisch wie folgt darstellen:

\begin{tabular}{c|c|c|c|} 
& 4 Wochen & 8 Wochen & 6 Monate \\
\hline $\begin{array}{c}\text { Experimental- } \\
\text { Gruppe }\end{array}$ & Kontrollzeitraum & $\begin{array}{c}\text { t1 } \\
\text { Psychoedukatives } \\
\text { Bewegungsprogramm }\end{array}$ & Follow-up \\
\hline $\begin{array}{c}\text { Kontroll- } \\
\text { Gruppe }\end{array}$ & Kontrollzeitraum & Fitnessprogramm & Follow-up
\end{tabular}

t $0=4$ Wochen vor Programmbeginn

$\mathrm{t} 1=$ Programmbeginn

t $2=8$ Wochen nach Programmbeginn (Programmende)

t3 $=6$ Monate nach Ende des Programms

Abbildung 11: Graphische Darstellung des Untersuchungsdesigns

Bei dem Ersttermin (t0) 4 Wochen vor Kursbeginn erhielten alle Teilnehmer ein ausführliches Informationsgespräch über Ziele und Inhalte der Studie. Des Weiteren wurden die soziodemographischen Daten sowie die Gesundheitsfragen erfasst.

Nach Vorlage der Einverständniserklärung wurden die Bioimpedanzmessung sowie ein Teil der sportmotorischen Testung (fahrradergometrische Untersuchung) durchgeführt. Die restlichen sportmotorischen Tests fanden $\mathrm{zu}$ einem weiteren vereinbarten Zeitpunkt in einer Kleingruppe mit anderen Teilnehmern zusammen statt. $\mathrm{Zu}$ diesem Testzeitpunkt (t0) wurden auch die in Tabelle 15 aufgeführten Fragebogen ausgefüllt.

Dieses Verfahren wiederholte sich in gleicher Weise bei den folgenden Messzeitpunkten (t1, t2, t3).

Aufgrund des hohen Testumfangs, verursacht insbesondere durch die genaue und aufwendige Testung der Ausdauerleistungsfähigkeit durch fahrradergometrische Belastungsuntersuchung und den Walkingtest wurden, zu jedem Testzeitpunkt zwei Testtermine vereinbart. 


\subsubsection{Ablauf der Datenerhebung}

Die folgende Tabelle gibt den genauen Ablauf der Datenerhebung wieder. Hier kann entnommen werden, welches Messinstrument zu welchem Messzeitpunkt eingesetzt wurde.

Tabelle 15: Chronologie der Datenerhebung

\begin{tabular}{|l|l|l|l|}
\hline t0 & t1 & t2 & t3 (Katamnese) \\
\hline $\begin{array}{l}\text { 4 Wochen vor } \\
\text { Programmbeginn }\end{array}$ & bei Programmbeginn & $\begin{array}{l}\text { 8 Wochen nach } \\
\text { Beginn } \\
\text { Programmende }\end{array}$ & $\begin{array}{l}\text { 6 Monate } \\
\text { nach Programmende }\end{array}$ \\
\hline SF-36 & SF-36 & SF-36 & SF-36 \\
\hline FKKS & FKKS & FKKS & FKKS \\
\hline BDI & BDI & BDI & BDI \\
\hline SIAB & SIAB & SIAB & SIAB \\
\hline $\begin{array}{l}\text { Fragebogen zur } \\
\text { körperl.-sportl. } \\
\text { Aktivität }\end{array}$ & $\begin{array}{l}\text { Fragebogen zur } \\
\text { körperl.-sportl. }\end{array}$ & $\begin{array}{l}\text { Fragebogen zur } \\
\text { körperl.-sportl. } \\
\text { Aktivität }\end{array}$ & $\begin{array}{l}\text { Fragebogen zur } \\
\text { körperl.-sportl. } \\
\text { Aktivität }\end{array}$ \\
\hline BIA- Messung & BIA- Messung & BIA- Messung & BIA- Messung \\
\hline Fahrraderg. Test & Fahrraderg. Test & Fahrraderg. Test & Fahrraderg. Test \\
\hline Walkingtest & Walkingtest & Walkingtest & Walkingtest \\
\hline BKT & BKT & BKT & BKT \\
\hline Krafttest & Krafttest & Krafttest & Krafttest \\
\hline
\end{tabular}

\subsubsection{Beschreibung der Stichprobe}

Die wichtigsten soziodemographischen Daten im Gruppenvergleich sind in Tabelle 15 aufgeführt. Die Vergleichbarkeit der Gruppen wurde mittels des $\mathrm{Chi}^{2}$-Tests für nonparametrische und bei intervallskalierten Daten mittels des t-Tests überprüft. Die Normalverteilung wurde anhand des Kolmogorov-Smirnov-Tests (Bortz, Lienert \& Boehnke, 1990) überprüft. 
Tabelle 16: Soziodemographische und anthropometrische Daten im Gruppenvergleich

\begin{tabular}{|c|c|c|c|c|}
\hline & Insgesamt & Versuchsgruppe & Kontrollgruppe & P-Wert \\
\hline Stichprobengröße (N) & $\begin{array}{l}\mathrm{N}=58 \\
\text { Frauen }=53 \\
\text { Männer }=5\end{array}$ & $\begin{array}{l}\mathrm{n}=30 \\
\text { Frauen }=28 \\
\text { Männer }=2\end{array}$ & $\begin{array}{l}\mathrm{n}=28 \\
\text { Frauen }=25 \\
\text { Männer }=3\end{array}$ & 0,66 \\
\hline $\begin{array}{l}\text { Alter in Jahren } \\
\text { Gruppenmittelwert (M) } \\
\text { Standardabweichung (SD) }\end{array}$ & $\begin{array}{l}44,34 \\
10,34 \\
\end{array}$ & $\begin{array}{l}44,93 \\
10,47\end{array}$ & $\begin{array}{l}43,71 \\
10,37\end{array}$ & 0,65 \\
\hline $\begin{array}{l}\text { Beruf } \\
\text { ungelernt } \\
\text { qualifiziert, einfach } \\
\text { qualifiziert, mittel } \\
\text { qualifiziert, leitend } \\
\text { selbständig, Kleinbetrieb } \\
\text { in Ausbildung } \\
\text { Hausfrau } \\
\text { Rente }\end{array}$ & $\begin{array}{l}0 \\
2 \\
35 \\
4 \\
1 \\
1 \\
11 \\
4\end{array}$ & $\begin{array}{l}0 \\
2 \\
16 \\
3 \\
1 \\
1 \\
6 \\
1\end{array}$ & $\begin{array}{l}0 \\
0 \\
19 \\
1 \\
0 \\
0 \\
5 \\
3\end{array}$ & 0,39 \\
\hline $\begin{array}{l}\text { Familienstand } \\
\text { ledig } \\
\text { verheiratet } \\
\text { geschieden }\end{array}$ & $\begin{array}{l}13 \\
40 \\
5\end{array}$ & $\begin{array}{l}5 \\
22 \\
3 \\
\end{array}$ & $\begin{array}{l}8 \\
18 \\
2 \\
\end{array}$ & 0,54 \\
\hline $\begin{array}{l}\text { Körpergewicht in kg } \\
\text { Gruppenmittelwert (M) } \\
\text { Standardabweichung (SD) }\end{array}$ & $\begin{array}{l}110,33 \\
16,20\end{array}$ & $\begin{array}{l}108,03 \\
10,87\end{array}$ & $\begin{array}{l}112,80 \\
20,36\end{array}$ & 0,26 \\
\hline $\begin{array}{l}\text { BMI in } \mathrm{kg} / \mathrm{m}^{2} \\
\text { Gruppenmittelwert (M) } \\
\text { Standardabweichung (SD) }\end{array}$ & $\begin{array}{l}39,82 \\
4,49 \\
\end{array}$ & $\begin{array}{l}38,84 \\
2,91\end{array}$ & $\begin{array}{l}40,87 \\
5,59\end{array}$ & 0,09 \\
\hline
\end{tabular}

Tabelle 17: Zusätzliche Informationen, Medikation und Vorbehandlung

\begin{tabular}{|l|l|l|l|l|}
\hline & Insgesamt & Versuchsgruppe & Kontrollgruppe & P-Wert \\
\hline Stichprobengröße (N) & $\begin{array}{l}\mathrm{N}=58 \\
\text { Frauen = 53 } \\
\text { Männer = 5 }\end{array}$ & $\begin{array}{l}\mathrm{n}=30 \\
\text { Frauen =28 } \\
\text { Männer =2 }\end{array}$ & $\begin{array}{l}\mathrm{n}=28 \\
\text { Frauen = 25 } \\
\text { Männer = 3 }\end{array}$ & \\
\hline Raucher & 16 & 5 & 11 & 0,8 \\
\hline $\begin{array}{l}\text { Blutdrucksenkende } \\
\text { Medikation }\end{array}$ & 7 & 4 & 3 & 1,0 \\
\hline $\begin{array}{l}\text { Psychotherapeutische } \\
\text { Behandlung }\end{array}$ & 9 & 3 & 6 & 0,29 \\
\hline $\begin{array}{l}\text { Stationäre Behandlung } \\
\text { wegen Übergewichts }\end{array}$ & 14 & 5 & 9 & 0,22 \\
\hline
\end{tabular}


Es wurden keine signifikanten Gruppenunterschiede gefunden, so dass eine gute Vergleichbarkeit der Gruppen vorausgesetzt wird.

Alle 58 Probanden absolvierten je nach Einteilung in Experimental- oder Kontrollgruppe das der Gruppe entsprechende Bewegungsprogramm.

3 Probandinnen haben die Teilnahme an dem Programm vorzeitig beendet. Alle 3 Probandinnen gehörten der Experimentalgruppe an. Die erste Teilnehmerin brach nach der 10. Sitzung (2/3 des Programms) ab. Ursache für den Abbruch waren orthopädische Probleme, die jedoch nicht mit dem Programm in Verbindung standen. Die anderen 2 Teilnehmerinnen brachen ihre Teilnahme jeweils in der 1. und 2. Sitzung des Bewegungsprogramms ab. Als Grund dafür gab eine Probandin an, dass das Programm nicht ihren Vorstellungen entspräche. Die andere Probandin gab an, an dem Bewegungsprogramm aus familiären Gründen und Zeitmangel nicht weiter teilnehmen zu können.

Weitere 5 Personen absolvierten erfolgreich den Messzeitpunkt t0, nahmen jedoch an dem nächsten Messzeitpunkt t1 und am Programm nicht mehr teil.

Eine Probandin riss sich in der Testung t0 beim Koordinationstest die Achillessehne, und konnte aufgrund der langwierigen Behandlung nicht am Bewegungsprogramm teilnehmen.

\subsection{Untersuchungsdurchführung}

Die Untersuchung wurde im Zeitraum zwischen Februar 2002 und März 2004 in der Klinik Roseneck durchgeführt.

Die beschriebenen Untersuchungsverfahren wurden 4 Wochen vor Programmbeginn, innerhalb der erste Woche der ambulanten bewegungstherapeutischen Behandlung, nach achtwöchiger Behandlung, d.h. bei Ende des psychoedukativen oder fitnessorientierten Programms, und 6 Monate nach Ende der Bewegungsprogramme eingesetzt.

Alle Untersuchungen wie auch das Verteilen der Fragebögen wurden vom Verfasser selbst oder einer in der Klinik Roseneck angestellten Sporttherapeutin durchgeführt. Das Wiegen in Verbindung mit der Bestimmung der Körperzusammensetzung (BIAMessung) und die fahrradergometrische Belastungsuntersuchung wurden als Einzeluntersuchungen in verschiedenen Räumen der Klinik auf dem Körperzusammenset- 
zungsanalysegerät bzw. auf dem computergesteuerten Fahrradergometer nachmittags in der Zeit zwischen 15:00 und 19:00 vorgenommen.

Die sportmotorischen Tests fanden samstags vormittags in kleinen Gruppen in der Gymnastikhalle (Aula) der Klinik statt. Für den Walkingtest wurde auf einer $2 \mathrm{~km}$ langen, ebenen und flachen Strecke in unmittelbarer Kliniknähe durchgeführt.

Die Fragebögen wurden von den Teilnehmern selbständig ausgefüllt.

Die soziodemographischen Daten wie auch Gesundheitsfragen zur Stichprobenbeschreibung wurden von dem Verfasser bei dem Ersttermin erhoben. Bei schweren Begleiterkrankungen und auffälligen medizinische Fragen bezüglich der Probanden konnte nach Bedarf Rücksprache mit dem das Projekt betreuenden Chefarzt gehalten werden.

\subsection{Datenauswertung}

Die statistische Auswertung der erhobenen Daten wurde mit dem StatistikComputerprogrammpaket SPSS/PC 11.5 geleistet. Die Grafiken wurden mit dem Berechnungsprogramm „Microsoft Excel“ erstellt.

\subsubsection{Statistische Verfahren}

Als deskriptive Werte wurden der Median, der arithmetische Mittelwert (M) und die Standardabweichung (SD) berechnet. Für inferenzstatistische Fragen wurden der UTest von Mann-Whitney, der Wilcoxon-Test, t-Tests, einfaktorielle und multifaktorielle Varianzanalysen durchgeführt.

Zusätzlich wurde im Rahmen der Varianzanalyse das spezifische Effektstärkemaß $\operatorname{Eta}^{2}\left(\eta^{2}\right)$ berechnet, das den Anteil der erklärten „treatment“- Varianz an der Gesamtvarianz wiedergibt. Nach Cohen (1988) kann ein $\eta^{2}$ von 0,01 als kleiner Effekt, $\eta^{2}$ von 0,059 als mittlerer Effekt und ein $\eta^{2}$ von 0,138 als großer Effekt bezeichnet werden.

Zur Überprüfung der Normalverteilung wurde der Kolmogorov-Smirnov-Test (Bortz, Lienert \& Boehnke, 1990) verwendet. Die Varianzhomogenität wurde mit Hilfe des Levene-Tests geprüft.

In der Ergebnisdarstellung werden Daten, die nicht normalverteilt sind, als solche ausgewiesen. 
Liegt eine Normalverteilung vor, so wird dieses nicht extra erwähnt.

Die zugrunde liegenden statistischen Verfahren werden bei Willimczik (1997) ausführlich beschrieben. Eine ausführliche SPSS- Nutzungsbeschreibung findet sich bei Bühl und Zöfel (2002).

\subsubsection{Fehlende Daten}

Ein vollständiger Datensatz bei t0 war Voraussetzung, um in die Studie aufgenommen zu werden.

Die Datenausfälle zwischen t1 und t3 werden in der Datenpräsentation für die einzelnen Messzeitpunkte und Instrumente dargestellt.

Zum Katamnesezeitpunkt lagen 58 (100\%) von 58 Fragebogenpaketen vor und 56 (96\%) Teilnehmer konnten für eine vollständige Testung gewonnen werden. Die im Verlauf der Behandlung fehlenden Daten wurden im Sinne eines „Intent to Treat“ (ITT)-Vorgehens durch die Daten der Aufnahme ersetzt. Fehlende Werte werden so in konservativer Weise als unveränderte Werte betrachtet. 



\section{Untersuchungsergebnisse}

Die Auswertung der Daten und die Darstellung der Ergebnisse orientieren sich an den Fragestellungen der Untersuchung (siehe Kap. 5).

Dem Usus der Sozialwissenschaften entsprechend werden Unterschiede mit einer Irrtumswahrscheinlichkeit von $\mathrm{p}<.05$ als signifikant, $\mathrm{p}<.01$ als sehr signifikant und $\mathrm{p}<.001$ als hoch signifikant interpretiert. Eine Irrtumswahrscheinlichkeit von $\mathrm{p}<.1$ weist ein nicht signifikantes, aber tendenzielles Unterschiedsniveau aus (Bortz, 1985).

\subsection{Körpergewicht, Body Mass Index und Körperzusammensetzung}

Bezüglich der Körpergewichtsentwicklung, der fettfreien Körpermasse in Kilogramm, der Muskelmasse und des Körperwassers in Kilogramm gibt es zu keinem der 4 Messzeitpunkte signifikante Gruppenunterschiede.

Gruppenunterschiede gibt es bzgl. des BMI und der Körperfettanteilmessungen.

Es zeichnet sich zunächst eine Tendenz von dem Testzeitpunkt $\mathrm{t} 1$ bis $\mathrm{t} 2$ für beide Messparameter ab. In der Katamnese (t2-t3) ist ein signifikanter Unterschied bei beiden Parametern zu finden.

Die Daten des Körpergewichts und des BMI sind in Tabelle 18 und Abbildung 11 dargestellt.

Tabelle 18: Mittelwerte und Standardabweichungen des Körpergewichts und Body Mass Indexes der beiden Gruppen zu allen Messzeitpunkten, t0 = bei Aufnahme, $t 1=$ 4 Wochen nach Aufnahme (Kursbeginn), t2 $=8$ Wochen nach Kursbeginn (Kursende), $t 3=6$ Monaten nach Kursende

\begin{tabular}{|c|c|c|c|c|}
\hline & $\mathrm{t} 0$ & $\mathrm{t} 1$ & $\mathrm{t} 2$ & $\mathrm{t} 3$ \\
\hline Körpergewicht in kg der & & & & \\
Experimentalgruppe (N=30) & 108,03 & 107,59 & 105,58 & 106,17 \\
Gruppenmittelwert (M) & $\pm 10,87$ & $\pm 11,33$ & $\pm 11,29$ & $\pm 12,69$ \\
Standardabweichung (SD) & & & & \\
\hline BMI in kg/m der & & 38,68 & 37,95 & 38,14 \\
Experimentalgruppe (N=30) & 38,84 & $\pm 2,92$ & $\pm 2,87$ & $\pm 3,30$ \\
Gruppenmittelwert (M) & $\pm 2,91$ & \pm 2, \\
Standardabweichung (SD) & &
\end{tabular}

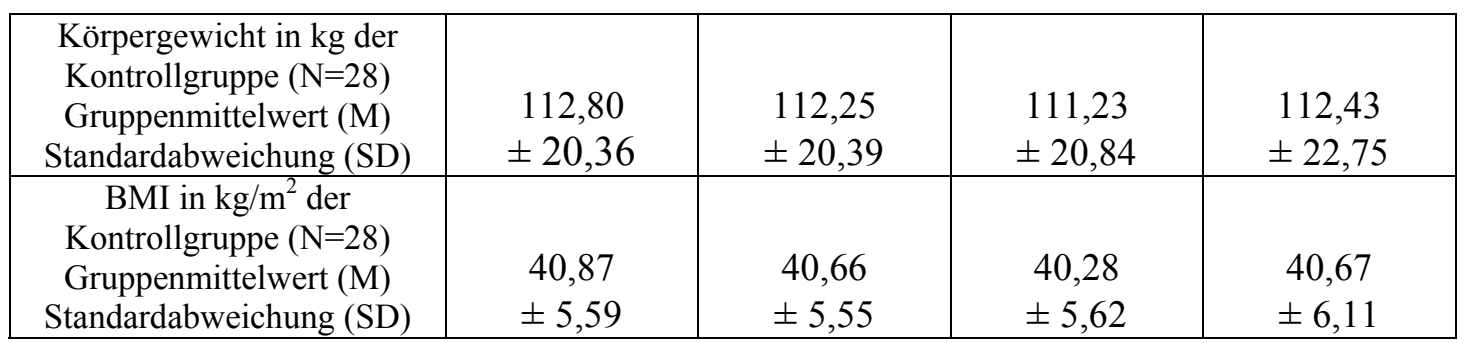




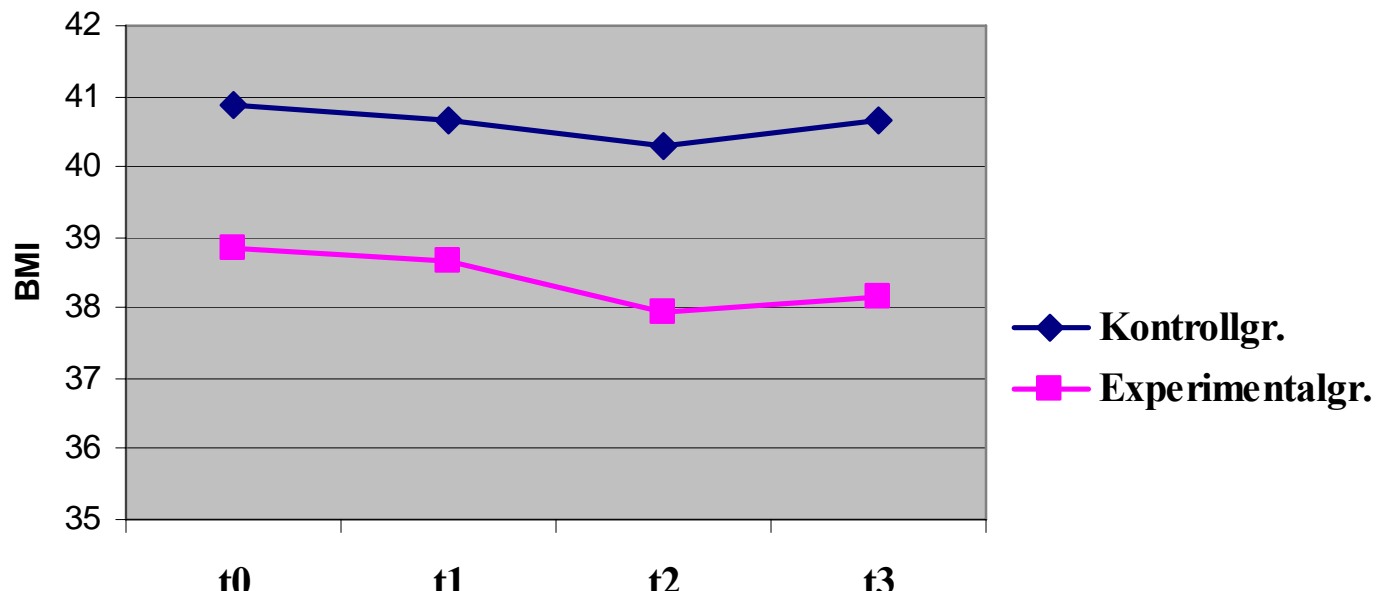

Abbildung 12: Verlauf des BMI der Experimental $(n=30)$ und Kontrollgruppe $(n=28)$ über 4 Messzeitpunkte im Gruppenvergleich

Die folgende Tabelle fasst die Ergebnisse der varianzanalytischen Testung zusammen:

Tabelle 19: Signifikante Ergebnisse der zweifaktoriellen Varianzanalyse mit Messwiederholungen für den $B M I(N=58)$

\begin{tabular}{|l|l|l|l|}
\hline AV & Haupteffekt: Zeit & Signifikanz & Effektstärke $\left(\mathrm{Eta}^{2}\right)$ \\
\hline BMI (t0-t1) & $\mathrm{F}=7,18$ & $\mathrm{p}=.01$ & $\eta^{2}=.114$ \\
\hline BMI (t1- t2) & $\mathrm{F}=20,20$ & $\mathrm{p}=.000$ & $\eta^{2}=.265$ \\
\hline & Haupteffekt: Gruppe & & \\
\hline BMI (t2-t3) & $\mathrm{F}=4,09$ & $\mathrm{p}=.048$ & $\eta^{2}=.068$ \\
\hline
\end{tabular}

Das Ausgangsgewicht der Kontrollgruppe liegt um 4,77 $\mathrm{kg}$ höher als das der Experimentalgruppe. Dieser Gruppenunterschied zum ersten Messzeitpunkt ist jedoch nicht signifikant (T-Test; $\mathrm{p}=.26)$.

Bei beiden Gruppen findet während der Baseline- und Treatmentphase eine Gewichtsreduktion statt. Dies ist nach der zweifaktoriellen Varianzanalyse mit Messwiederholungen ein signifikanter Zeiteffekt.

Das Körpergewicht steigt nach der Behandlungsphase während der Katamnese für beide Gruppen gering an.

Das niedrigste Gewicht eines Teilnehmers bei Aufnahme war 83,20 (BMI 34,95) und das Maximum $165 \mathrm{~kg}$ (BMI 57,11).

Während der Baseline Periode nehmen die Experimentalgruppe $(0,44 \mathrm{~kg})$ und Kontrollgruppe $(0,55 \mathrm{~kg}) \mathrm{ab}$. 
Während des Programms nehmen die Teilnehmer der Experimentalgruppe mit durchschnittlich 2,01 kg das meiste an Gewicht ab. Die Gewichtsreduktion in der Kontrollgruppe beträgt durchschnittlich $1,02 \mathrm{~kg}$. Dieser Unterschied in der Gewichtsreduktion ist nicht signifikant.

Die maximale Gewichtsreduktion über den Zeitraum des Bewegungsprogramms (t1t2) ist für die Experimentalgruppe 9,5 kg und für die Kontrollgruppe 7,0 kg.

22, 4 \% aller Teilnehmer verloren während der Treatmentzeit mehr als 3,2 kg.

Über den Zeitraum der Follow-up-Periode nimmt das Gewicht bei der Experimentalgruppe um 0,59 $\mathrm{kg}$ und bei der Kontrollgruppe um 1,2 kg zu. Nach der letzten Messung liegt das Gewicht der Experimentalgruppe durchschnittlich um 1,86 $\mathrm{kg}$ niedriger als bei Studieneinschluß, während es bei der Kontrollgruppe mit einer Gewichtsreduktion von $0,37 \mathrm{~kg}$ das Aufnahmegewicht fast wiedererreicht.

Die maximale Gewichtsabnahme über die Messzeitpunkte t1 -t3 (Treatmentbeginn Follow-up) beträgt 19,70 kg (Teilnehmerin der Experimentalgruppe). Die maximale Gewichtszunahme von 14,10 kg zeigt ein Teilnehmer der Kontrollgruppe.

Während die fettfreie Körpermasse über die gesamte Zeit der Untersuchung für beide Gruppen stabil bleibt, ist es der Körperfettanteil, der die Gewichtsreduktion bzw. Gewichtszunahme bedingt.

Die Daten der fettfreien Körpermasse und des Körperfettanteils sind in der folgenden Tabelle dargestellt.

Tabelle 20: Mittelwerte und Standardabweichungen der fettfreien Körpermasse und des Körperfettanteils in $\mathrm{kg}$ der beiden Gruppen zu allen Messzeitpunkten, t0 = bei Aufnahme, $t 1=4$ Wochen nach Aufnahme (Kursbeginn), t2 = 8 Wochen nach Kursbeginn (Kursende), $t 3=6$ Monate nach Kursende

\begin{tabular}{|c|c|c|c|c|}
\hline & $\mathrm{t} 0$ & $\mathrm{t} 1$ & $\mathrm{t} 2$ & $\mathrm{t} 3$ \\
\hline $\begin{array}{c}\text { Fettfreie Körpermasse in kg der } \\
\text { Experimentalgruppe (n=30) }\end{array}$ & & & & \\
Gruppenmittelwert (M) & 60,64 & 61,08 & 61,17 & 60,80 \\
Standardabweichung (SD) & $\pm 8,03$ & $\pm 8,19$ & $\pm 7,87$ & $\pm 8,33$ \\
\hline Körperfettanteil der & & & & \\
Experimentalgruppe (n=30) & 47,41 & 46,52 & 44,41 & 45,38 \\
Gruppenmittelwert (M) & $\pm 6,17$ & $\pm 6,29$ & $\pm 6,03$ & $\pm 7,05$ \\
Standardabweichung (SD) & & & & \\
\hline Fettfreie Körpermasse in kg der & & & & \\
Kontrollgruppe (n=28) & 61,47 & 61,37 & 61,33 & 61,01 \\
Gruppenmittelwert (M) & $\pm 10,12$ & $\pm 10,37$ & $\pm 10,84$ & $\pm 10,31$ \\
Standardabweichung (SD) & & & & \\
\hline Körperfettanteil der & & & & \\
Kontrollgruppe (n=28) & 51,33 & 50,87 & 49,90 & 51,42 \\
Gruppenmittelwert (M) & $\pm 12,99$ & $\pm 12,53$ & $\pm 12,79$ & $\pm 15,06$ \\
Standardabweichung (SD) & & & & \\
\hline
\end{tabular}


Der Körperfettanteil reduziert sich über die Baselineperiode (t0-t1) für die Experimentalgruppe um $0,880 \mathrm{~kg}$ bei einer Gesamtkörpergewichtsreduktion von 0,437 kg (parallel zur Körperfettreduktion wurde Muskelmasse aufgebaut). Die Teilnehmer der Kontrollgruppe verlieren bei einer Gesamtgewichtsabnahme von $0,553 \mathrm{~kg}$ an Fett $0,460 \mathrm{~kg}$.

Die Experimentalgruppe verliert während des Treatments 2,11 kg an Fettmasse bei einer Gesamtgewichtsreduktion von 2,01 kg. In der gleichen Zeit verliert die Kontrollgruppe $0,96 \mathrm{~kg}$ an Fettmasse. Der annähernd gleiche Wert der Gesamtgewichtsreduktion von $1,02 \mathrm{~kg}$ belegt, dass es nicht wie bei der Experimentalgruppe zu einem Aufbau von Muskelmasse kommt.

Über den Zeitraum der Katamnese nimmt der Körperfettanteil bei der Experimentalgruppe um $0,970 \mathrm{~kg}$ bei einer Gesamtgewichtszunahme von 0,593 $\mathrm{kg}$ zu. Auch bei der Kontrollgruppe übersteigt der Zuwachs an Körperfett mit 1,52 kg leicht die Gesamtgewichtszunahme von 1,2 kg. Somit wird ein geringer Umbau von Muskelmasse in Fettmasse belegt.

Die folgende Abbildung stellt den Verlauf der fettfreien Körpermasse und Fettmasse der beiden Gruppen über die gesamte Studienzeit dar.

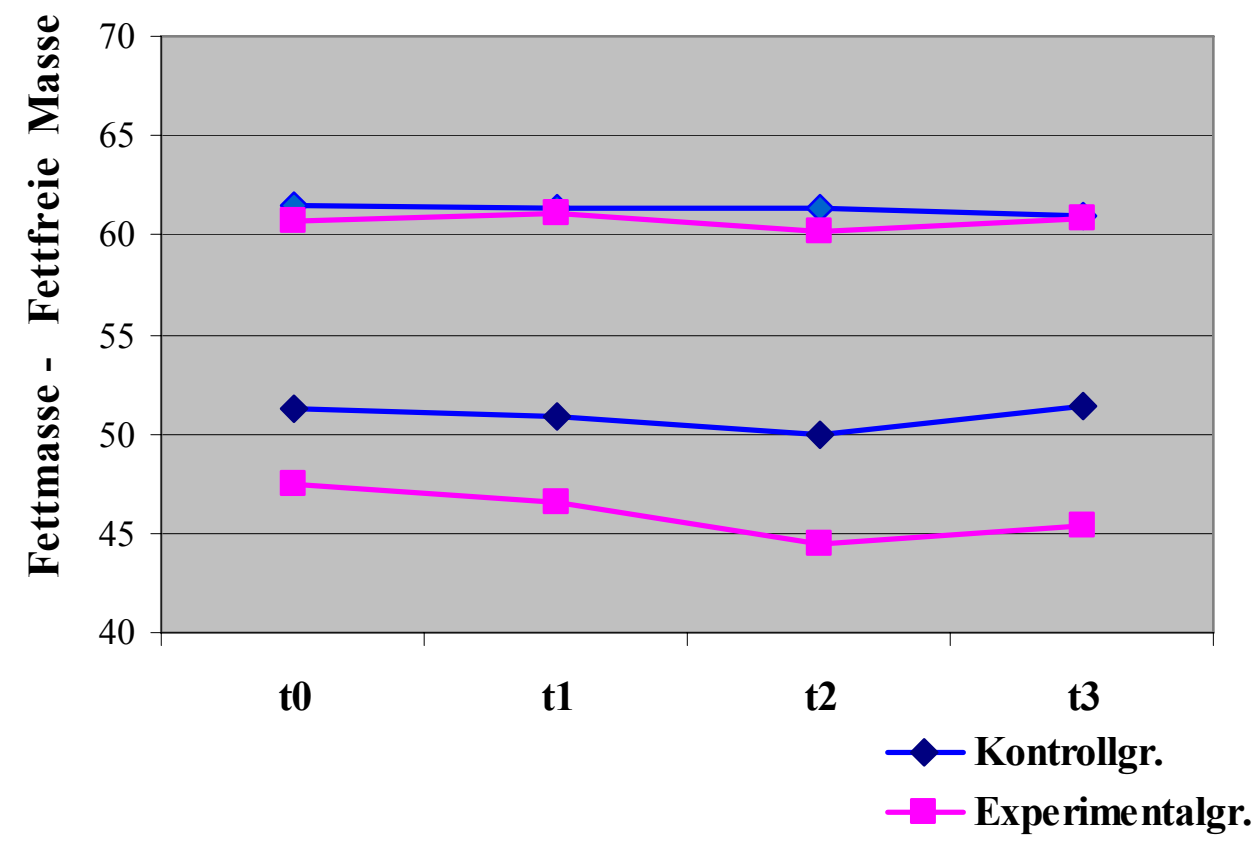

Abbildung 13: Verlauf der fettfreien Körpermasse und Fettmasse der Experimentalgruppe $(n=30)$ und Kontrollgruppe $(n=28)$ anhand der Mittelwerte über 4 Messzeitpunkte im Gruppenvergleich 
Die folgende Tabelle fasst die Ergebnisse der varianzanalytischen Testung zusammen:

Tabelle 21: Signifikante Ergebnisse der zweifaktoriellen Varianzanalyse mit Messwiederholungen für den Körperfettanteil $(N=58)$

\begin{tabular}{|l|l|l|l|}
\hline AV & Haupteffekt : Zeit & Signifikanz & Effektstärke (Eta $\left.{ }^{2}\right)$ \\
\hline Körperfettanteil (t0-t1) & $\mathrm{F}=7,79$ & $\mathrm{p}=.007$ & $\eta^{2}=.122$ \\
\hline Körperfettanteil (t1- $\mathrm{t} 2)$ & $\mathrm{F}=32,31$ & $\mathrm{p}=.000$ & $\eta^{2}=.366$ \\
\hline Körperfettanteil (t2- t3) & $\mathrm{F}=5,69$ & $\mathrm{p}=.02$ & $\eta^{2}=.092$ \\
\hline & Interaktion: Gruppe* Zeit & & \\
\hline Körperfettanteil (t1-t2) & $\mathrm{F}=4,46$ & $\mathrm{p}=.039$ & $\eta^{2}=.074$ \\
\hline & Haupteffekt : Gruppe & & \\
\hline Körperfettanteil (t2-t3) & $\mathrm{F}=4,28$ & $\mathrm{p}=.043$ & $\eta^{2}=.071$ \\
\hline
\end{tabular}

Die Interaktion Gruppe*Zeit ist signifikant. Die Fettreduktion der Experimentalgruppe über die Interventionszeit ist statistisch deutlicher als die der Kontrollgruppe.

\subsection{Erfassung der gesundheitsbezogenen Lebensqualität}

Die gesundheitsbezogene Lebensqualität wird anhand der Ergebnisse aus den acht Subskalen des SF-36 erfasst. Die Werte werden als transformierte Skalenwerte dargestellt. Die Transformation formt den niedrigsten Wert in 0 und den höchst möglichen Wert in $100 \mathrm{um}$. Höhere Werte entsprechen einem generell besseren Gesundheitszustand.

Die folgende Tabelle zeigt die Veränderung der Mittelwerte der acht Subskalen des SF-36 während der gesamten Studienzeit. 
Tabelle 22: Mittelwerte und Standardabweichungen der SF-36 Skalenwerte der beiden Gruppen zu allen Messzeitpunkten, t0 = bei Aufnahme, $t 1=4$ Wochen nach Aufnahme (Kursbeginn), t2 $=8$ Wochen nach Kursbeginn (Kursende), $t 3=6$ Monate nach Kursende

\begin{tabular}{|c|c|c|c|c|}
\hline Experimentalgruppe (n=30) & $\mathrm{t} 0$ & $\mathrm{t} 1$ & $\mathrm{t} 2$ & $\mathrm{t} 3$ \\
\hline $\begin{array}{c}\text { Körperliche Funktionsfähigkeit } \\
\text { Arithmetisches Mittel } \\
\text { Standardabweichung (SD) }\end{array}$ & $\begin{array}{c}71,37 \\
\pm 20,61\end{array}$ & $\begin{array}{c}73,16 \\
\pm 21,55\end{array}$ & $\begin{array}{c}84,66 \\
\pm 18,75\end{array}$ & $\begin{array}{c}82,33 \\
\pm 18,60\end{array}$ \\
\hline $\begin{array}{c}\text { Körperliche Rollenfunktion } \\
\text { Arithmetisches Mittel } \\
\text { Standardabweichung (SD) }\end{array}$ & $\begin{array}{c}74,72 \\
\pm 35,38\end{array}$ & $\begin{array}{c}82,50 \\
\pm 26,38\end{array}$ & $\begin{array}{c}87,50 \\
\pm 28,42\end{array}$ & $\begin{array}{c}85,00 \\
\pm 28,31\end{array}$ \\
\hline $\begin{array}{c}\text { Körperliche Schmerzen } \\
\text { Arithmetisches Mittel }\end{array}$ & $\begin{array}{c}70,34 \\
\pm 24,55\end{array}$ & $\begin{array}{c}70,16 \\
\pm 24,98\end{array}$ & $\begin{array}{c}81,00 \\
\pm 22,65\end{array}$ & $\begin{array}{c}77,03 \\
\pm 25,07\end{array}$ \\
\hline $\begin{array}{c}\text { Standardabweichung (SD) } \\
\text { Allgemeine Gesundheit } \\
\text { Arithmetisches Mittel }\end{array}$ & $\begin{array}{c}65,89 \\
\text { Standardabweichung (SD) }\end{array}$ & $\begin{array}{c}66,66 \\
\pm 16,91\end{array}$ & $\begin{array}{c}75,1 \\
\pm 14,70\end{array}$ & $\begin{array}{c}69,00 \\
\pm 17,49\end{array}$ \\
\hline $\begin{array}{c}\text { Vitalität } \\
\text { Arithmetisches Mittel } \\
\text { Standardabweichung (SD) }\end{array}$ & $\begin{array}{c}56,20 \\
\pm 17,86\end{array}$ & $\begin{array}{c}59,16 \\
\pm 14,50\end{array}$ & $\begin{array}{c}71,16 \\
\pm 11,57\end{array}$ & $\begin{array}{c}65,16 \\
\pm 14,70\end{array}$ \\
\hline $\begin{array}{c}\text { Soziale Funktionsfähigkeit } \\
\text { Arithmetisches Mittel }\end{array}$ & $\begin{array}{c}80,60 \\
\pm 22,55\end{array}$ & $\begin{array}{c}84,58 \\
\pm 20,93\end{array}$ & $\begin{array}{c}90,00 \\
\pm 14,82\end{array}$ & $\begin{array}{c}87,50 \\
\pm 19,69\end{array}$ \\
\hline $\begin{array}{c}\text { Standardabweichung (SD) } \\
\text { Emotionale Rollenfunktion } \\
\text { Arithmetisches Mittel }\end{array}$ & $\begin{array}{c}86,20 \\
\pm 28,89\end{array}$ & $\begin{array}{c}84,44 \\
\pm 31,23\end{array}$ & $\begin{array}{c}96,66 \\
\pm 10,17\end{array}$ & $\begin{array}{c}93,88 \\
\pm 18,81\end{array}$ \\
\hline $\begin{array}{c}\text { Standardabweichung (SD) } \\
\text { Psychisches Wohlbefinden } \\
\text { Arithmetisches Mittel }\end{array}$ & $\begin{array}{c}70,89 \\
\text { Standardabweichung (SD) }\end{array}$ & $\begin{array}{c}72,00 \\
\pm 17,35\end{array}$ & $\begin{array}{c}79,73 \\
\pm 14,55\end{array}$ & $\begin{array}{c}78,80 \\
\pm 13,49\end{array}$ \\
\hline
\end{tabular}

\begin{tabular}{|c|c|c|c|c|}
\hline Kontrollgruppe $(n=28)$ & to & $\mathrm{t} 1$ & $\mathrm{t} 2$ & $\mathrm{t} 3$ \\
\hline $\begin{array}{c}\text { Körperliche Funktionsfähigkeit } \\
\text { Arithmetisches Mittel } \\
\text { Standardabweichung (SD) } \\
\end{array}$ & $\begin{array}{c}76,42 \\
\pm 19,57 \\
\end{array}$ & $\begin{array}{c}77,22 \\
\pm 20,58 \\
\end{array}$ & $\begin{array}{c}78,92 \\
\pm 20,51 \\
\end{array}$ & $\begin{array}{c}75,17 \\
\pm 22,66 \\
\end{array}$ \\
\hline $\begin{array}{l}\text { Körperliche Rollenfunktion } \\
\text { Arithmetisches Mittel } \\
\text { Standardabweichung (SD) }\end{array}$ & $\begin{array}{c}58,92 \\
\pm 45,24\end{array}$ & $\begin{array}{c}60,18 \\
\pm 45,07\end{array}$ & $\begin{array}{c}85,71 \\
\pm 31,49\end{array}$ & $\begin{array}{c}71,42 \\
\pm 42,87\end{array}$ \\
\hline $\begin{array}{l}\text { Körperliche Schmerzen } \\
\text { Arithmetisches Mittel } \\
\text { Standardabweichung (SD) }\end{array}$ & $\begin{array}{c}58,21 \\
\pm 29,57\end{array}$ & $\begin{array}{c}62,29 \\
\pm 30,16\end{array}$ & $\begin{array}{c}67,35 \\
\pm 27,29\end{array}$ & $\begin{array}{c}64,53 \\
\pm 30,76\end{array}$ \\
\hline $\begin{array}{l}\text { Allgemeine Gesundheit } \\
\text { Arithmetisches Mittel } \\
\text { Standardabweichung (SD) }\end{array}$ & $\begin{array}{c}64,54 \\
\pm 18,42\end{array}$ & $\begin{array}{c}62,00 \\
\pm 17,43\end{array}$ & $\begin{array}{c}67,17 \\
\pm 18,33\end{array}$ & $\begin{array}{c}62,64 \\
\pm 18,52\end{array}$ \\
\hline $\begin{array}{c}\text { Vitalität } \\
\text { Arithmetisches Mittel } \\
\text { Standardabweichung (SD) }\end{array}$ & $\begin{array}{c}45,57 \\
\pm 16,41\end{array}$ & $\begin{array}{c}51,66 \\
\pm 16,05\end{array}$ & $\begin{array}{c}55,89 \\
\pm 14,46\end{array}$ & $\begin{array}{c}50,35 \\
\pm 16,26\end{array}$ \\
\hline $\begin{array}{l}\text { Soziale Funktionsfähigkeit } \\
\text { Arithmetisches Mittel } \\
\text { Standardabweichung (SD) }\end{array}$ & $\begin{array}{r}73,66 \\
\pm 27,07\end{array}$ & $\begin{array}{c}81,94 \\
\pm 20,01\end{array}$ & $\begin{array}{c}85,26 \\
\pm 20,42\end{array}$ & $\begin{array}{c}79,46 \\
\pm 25,27\end{array}$ \\
\hline $\begin{array}{l}\text { Emotionale Rollenfunktion } \\
\text { Arithmetisches Mittel } \\
\text { Standardabweichung (SD) }\end{array}$ & $\begin{array}{c}83,33 \\
\pm 33,33\end{array}$ & $\begin{array}{c}83,35 \\
\pm 31,00\end{array}$ & $\begin{array}{c}78,57 \\
\pm 38,71\end{array}$ & $\begin{array}{c}78,57 \\
\pm 32,97\end{array}$ \\
\hline $\begin{array}{l}\text { Psychisches Wohlbefinden } \\
\text { Arithmetisches Mittel } \\
\text { Standardabweichung (SD) } \\
\end{array}$ & $\begin{array}{c}62,09 \\
\pm 17,11\end{array}$ & $\begin{array}{c}64,59 \\
\pm 17,67 \\
\end{array}$ & $\begin{array}{c}67,57 \\
\pm 17,56\end{array}$ & $\begin{array}{c}65,72 \\
\pm 19,37 \\
\end{array}$ \\
\hline
\end{tabular}


In der folgenden Abbildung sind die SF-36 Skalen beider Gruppen über die gesamte Studiendauer dargestellt.

\section{$\begin{array}{llllllllllll}0 & 10 & 20 & 30 & 40 & 50 & 60 & 70 & 80 & 90 & 100\end{array}$}

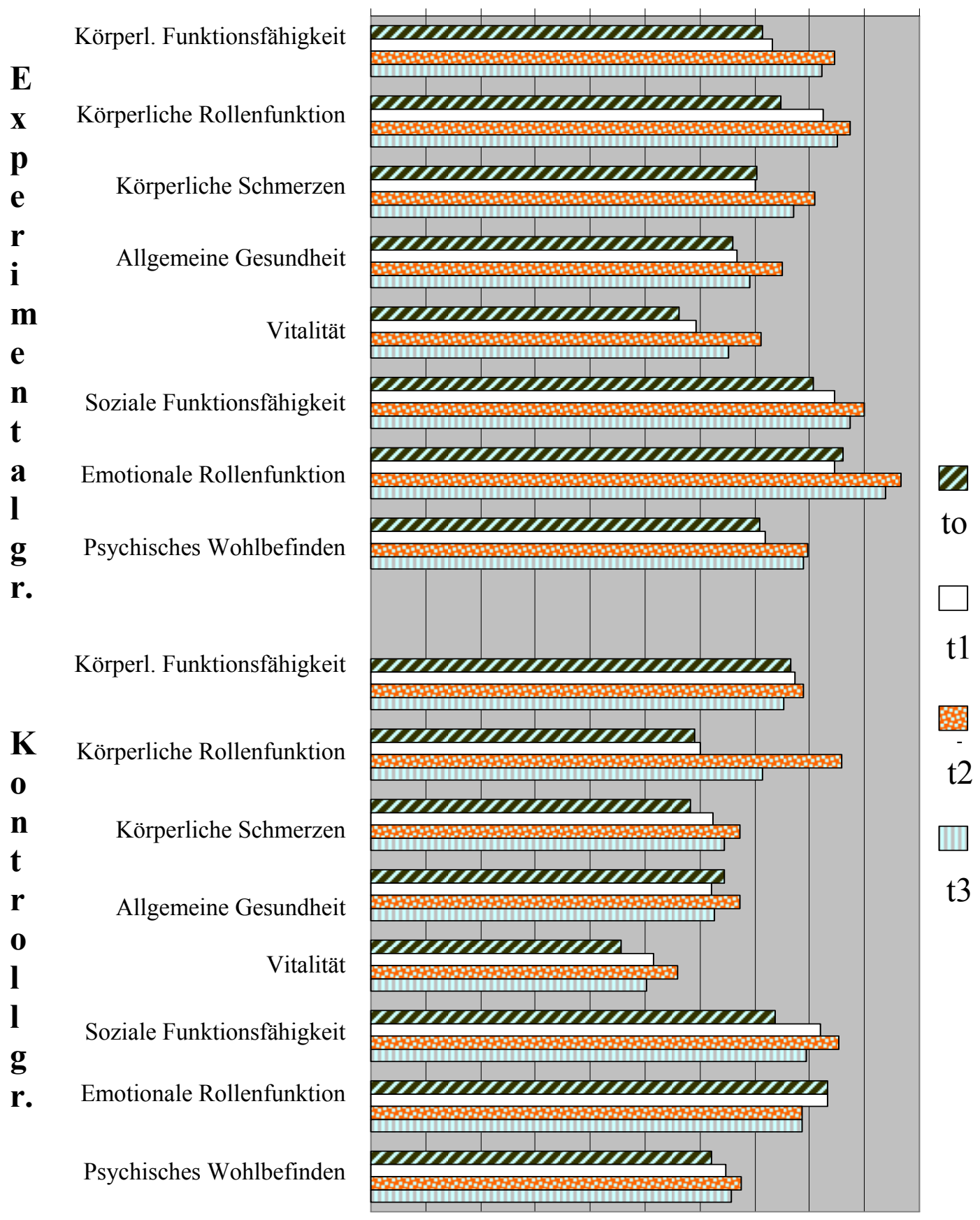

Abbildung 14: Veränderungen der SF-36 Skalenwerte der Experimentalgruppe $(\mathrm{n}=30)$ und Kontrollgruppe $(\mathrm{n}=28)$ über 4 Messzeitpunkte im Gruppenvergleich 
Über den Zeitraum der Baseline zeigen sich in Bezug auf sieben der acht Subskalen keine signifikanten Veränderungen. Der Wert der Subskala „Vitalität“ ist nach der varianzanalytischen Berechnung bezüglich der Faktoren Zeit und Gruppe signifikant angestiegen.

In der Treatmentphase sind signifikante Veränderungen in sieben von den insgesamt acht Subskalen des Instruments zu beobachten. Bei allen sieben Subskalen ist ein Anstieg der Werte und somit eine Verbesserung der Befindlichkeit zu sehen.

Die Skalawerte der Subskala „Emotionale Rollenfunktion“ verbessern sich bei der Experimentalgruppe und verschlechtern sich in der Kontrollgruppe. Diese Veränderungen sind jedoch bei beiden Gruppen nicht signifikant. Die geringsten Veränderungen der Skalenwerte in der Treatmentzeit werden für beide Gruppen im Bereich „Vitalität““ gemessen. Die höchsten Werte werden bei der Kontrollgruppe in der Subskala „Körperliche Rollenfunktion“ (15,53 Punkte) und bei der Experimentalgruppe bezüglich der „Emotionalen Rollenfunktion“ (12,22 Punkte) beobachtet.

Während der Katamnese ist ein Rückgang also eine Verschlechterung der Symptomatik bezüglich sieben der acht Subskalen belegt. Einzige Ausnahmen bilden für die Experimentalgruppe die Subskala „Körperliche Rollenfunktion“, deren Wert gleich bleibt, und für die Kontrollgruppe die Subskala „Emotionale Rollenfunktion“. Der höchste Abstieg findet sich für die Kontrollgruppe in der Subskala „Körperliche Rollenfunktion“ (14,29 Punkten) und bei der Experimentalgruppe in der Subskala „Allgemeine Gesundheit“ (6,1 Punkten). Am geringsten verändern sich in der Kontrollgruppe die Werte der Subskalen „Körperliche Rollenfunktion“ (Wert bleibt gleich) und „Psychisches Wohlbefinden“(1,85 Punkte). Bei der Experimentalgruppe ändert sich der Wert der Subskala „Psychisches Wohlbefinden“ mit 0,93 Punkten kaum.

Bezüglich der Subskalen „Körperliche Schmerzen“, „Allgemeine Gesundheit“, „Vitalität“, „Emotionale Rollenfunktion“ und „Psychisches Wohlbefinden“ sind die niedrigeren Werte bzgl. der Faktoren Zeit und Gruppe signifikant.

Die folgende Tabelle fasst alle signifikanten Ergebnisse der varianzanalytischen Testung zusammen: 
Tabelle 23: Ergebnisse der zweifaktoriellen Varianzanalyse mit Messwiederholungen für den SF-36 Skalenwert über 4 Messzeitpunkte

\begin{tabular}{|l|l|l|l|}
\hline AV & Haupteffekt : Zeit & Signifikanz & $\mathrm{Eta}^{2}$ \\
\hline Vitalität (t0-t) & $\mathrm{F}=4,26$ & $\mathrm{p}=.044$ & $\eta^{2}=.073$ \\
\hline & Haupteffekt : Gruppe & & \\
\hline Vitalität (t0-t1) & $\mathrm{F}=4,47$ & $\mathrm{p}=.039$ & $\eta^{2}=.076$ \\
\hline
\end{tabular}

\begin{tabular}{|l|l|l|l|}
\hline AV & Haupteffekt : Zeit & Signifikanz & $\mathrm{Eta}^{2}$ \\
\hline Körperl. Funktionsfähigkeit (t1-t2) & $\mathrm{F}=8,86$ & $\mathrm{p}=.004$ & $\eta^{2}=.139$ \\
\hline
\end{tabular}

\begin{tabular}{|l|l|l|l|}
\hline AV & Haupteffekt : Zeit & Signifikanz & $\mathrm{Eta}^{2}$ \\
\hline Körperl. Rollenfunktion (t1-t2) & $\mathrm{F}=12,89$ & $\mathrm{p}=.001$ & $\eta^{2}=.190$ \\
\hline & Interaktion: Gruppe* Zeit & & \\
\hline Körperl. Rollenfunktion (t1-t2) & $\mathrm{F}=6,37$ & $\mathrm{p}=.014$ & $\eta^{2}=.104$ \\
\hline
\end{tabular}

\begin{tabular}{|l|l|l|l|}
\hline AV & Haupteffekt : Zeit & Signifikanz & $\mathrm{Eta}^{2}$ \\
\hline Körperliche Schmerzen (t1-t2) & $\mathrm{F}=14,39$ & $\mathrm{p}=.000$ & $\eta^{2}=.207$ \\
\hline Allgemeine Gesundheit (t1-t2) & $\mathrm{F}=16,17$ & $\mathrm{p}=.000$ & $\eta^{2}=.227$ \\
\hline
\end{tabular}

\begin{tabular}{|l|l|l|l|}
\hline AV & Haupteffekt : Zeit & Signifikanz & $\mathrm{Eta}^{2}$ \\
\hline Vitalität (t1-t2) & $\mathrm{F}=18,89$ & $\mathrm{p}=.000$ & $\eta^{2}=.256$ \\
\hline & Haupteffekt : Gruppe & & \\
\hline Vitalität (t1-t2) & $\mathrm{F}=12,28$ & $\mathrm{p}=.001$ & $\eta^{2}=.183$ \\
\hline
\end{tabular}

\begin{tabular}{|l|l|l|l|}
\hline AV & Haupteffekt : Zeit & Signifikanz & Eta $^{2}$ \\
\hline Soziale Funktionsfähigkeit (t1-t2) & $\mathrm{F}=4,78$ & $\mathrm{p}=.033$ & $\eta^{2}=.080$ \\
\hline
\end{tabular}

\begin{tabular}{|l|l|l|l|}
\hline AV & Haupteffekt : Zeit & Signifikanz & $\mathrm{Eta}^{2}$ \\
\hline Psych. Wohlbefinden (t1-t2) & $\mathrm{F}=10,10$ & $\mathrm{p}=.002$ & $\eta^{2}=.155$ \\
\hline & Haupteffekt : Gruppe & & \\
\hline Psych. Wohlbefinden ( $\mathrm{t} 1-\mathrm{t} 2)$ & $\mathrm{F}=5,33$ & $\mathrm{p}=.025$ & $\eta^{2}=.088$ \\
\hline
\end{tabular}

\begin{tabular}{|l|l|l|l|}
\hline AV & Haupteffekt : Gruppe & Signifikanz & $\mathrm{Eta}^{2}$ \\
\hline Körperliche Schmerzen (t2-t3) & $\mathrm{F}=4,27$ & $\mathrm{p}=.043$ & $\eta^{2}=.071$ \\
\hline & Haupteffekt : Zeit & & \\
\hline Allgemeine Gesundheit (t2-t3) & $\mathrm{F}=8,19$ & $\mathrm{p}=.006$ & $\eta^{2}=.128$ \\
\hline
\end{tabular}

\begin{tabular}{|l|l|l|l|}
\hline AV & Haupteffekt : Zeit & Signifikanz & $\mathrm{Eta}^{2}$ \\
\hline Vitalität (t2-t3) & $\mathrm{F}=8,60$ & $\mathrm{p}=.005$ & $\eta^{2}=.133$ \\
\hline & Haupteffekt : Gruppe & & \\
\hline Vitalität (t2-t3) & $\mathrm{F}=21,90$ & $\mathrm{p}=.000$ & $\eta^{2}=.282$ \\
\hline
\end{tabular}

\begin{tabular}{|l|l|l|l|}
\hline AV & Haupteffekt : Gruppe & Signifikanz & Eta $^{2}$ \\
\hline Emotion. Rollenfunktion (t2-t3) & $\mathrm{F}=7,50$ & $\mathrm{p}=.008$ & $\eta^{2}=.118$ \\
\hline Psych. Wohlbefinden (t2-t3) & $\mathrm{F}=10,70$ & $\mathrm{p}=.002$ & $\eta^{2}=.160$ \\
\hline
\end{tabular}


Zusätzlich zur Berechnung der SF-36 Subskalen wurden die körperlichen und psychischen Summenskalen ausgewertet. Höhere Werte in den Summenskalen reflektieren einen besseren körperlichen und psychischen Gesundheitszustand.

Errechnete Summenskalenscores für die gesamte deutsche Normpopulation wie auch für einzelne Erkrankungsgruppen ermöglichen einen Vergleich mit den Summenskalenscores der Stichprobe.

Die folgende Tabelle und Abbildung zeigt die Veränderung der Mittelwerte der Summenskalen des SF-36 während der gesamten Studienzeit wie auch die Werte der deutschen Normstichprobe als Vergleichsgruppe.

Tabelle 24: Mittelwerte und Standardabweichungen der körperlichen und psychischen Summenskalen für die deutsche Normstichprobe und beide Gruppen der Untersuchung zu allen Messzeitpunkten, to $=$ bei Studieneinschluss, $t 1=$ Kursbeginn, $t 2=8$ Wochen nach Kursbeginn (Kursende), $t 3=6$ Monate nach Kursende

\begin{tabular}{|c|c|c|c|c|c|}
\hline $\begin{array}{c}\text { Experimentalgruppe } \\
(\mathbf{n}=\mathbf{3 0 )}\end{array}$ & t0 & t1 & t2 & t3 & $\begin{array}{c}\text { Deutsche } \\
\text { Normstichprobe } \\
\text { (N=2914) }\end{array}$ \\
\hline $\begin{array}{c}\text { Körperliche Summenskala } \\
\text { Arithmetisches Mittel } \\
\text { Standardabweichung (SD) }\end{array}$ & $\begin{array}{c}45,55 \\
\pm 9,88\end{array}$ & $\begin{array}{c}46,76 \\
\pm 9,65\end{array}$ & $\begin{array}{c}50,40 \\
\pm 9,49\end{array}$ & $\begin{array}{c}48,62 \\
\pm 8,75\end{array}$ & $\begin{array}{c}50,21 \\
\pm 10,24\end{array}$ \\
\hline $\begin{array}{c}\text { Psychische Summenskala } \\
\text { Arithmetisches Mittel } \\
\text { Standardabweichung (SD) }\end{array}$ & $\begin{array}{c}50,49 \\
\pm 10,60\end{array}$ & $\begin{array}{c}50,90 \\
\pm 9,89\end{array}$ & $\begin{array}{c}54,75 \\
\pm 6,42\end{array}$ & $\begin{array}{c}53,69 \\
\pm 6,56\end{array}$ & $\begin{array}{c}51,54 \\
\pm 8,14\end{array}$ \\
\hline
\end{tabular}

\begin{tabular}{|c|c|c|c|c|c|}
\hline $\begin{array}{c}\text { Kontrollgruppe } \\
\text { (n=28) }\end{array}$ & t0 & t1 & t2 & t3 & $\begin{array}{c}\text { Deutsche } \\
\text { Normstichprobe } \\
\text { (N=2914) }\end{array}$ \\
\hline $\begin{array}{c}\text { Körperliche Summenskala } \\
\text { Arithmetisches Mittel } \\
\text { Standardabweichung (SD) }\end{array}$ & $\begin{array}{c}44,17 \\
\pm 10,86\end{array}$ & $\begin{array}{c}44,43 \\
\pm 12,15\end{array}$ & $\begin{array}{c}48,68 \\
\pm 9,21\end{array}$ & $\begin{array}{c}45,73 \\
\pm 11,79\end{array}$ & $\begin{array}{c}50,21 \\
\pm 10,24\end{array}$ \\
\hline $\begin{array}{c}\text { Psychische Summenskala } \\
\text { Arithmetisches Mittel } \\
\text { Standardabweichung (SD) }\end{array}$ & $\begin{array}{c}46,28 \\
\pm 11,25\end{array}$ & $\begin{array}{c}48,37 \\
\pm 9,19\end{array}$ & $\begin{array}{c}48,07 \\
\pm 10,27\end{array}$ & $\begin{array}{c}47,30 \\
\pm 10,33\end{array}$ & $\begin{array}{c}51,54 \\
\pm 8,14\end{array}$ \\
\hline
\end{tabular}

Im Balkendiagramm wird die sich im Verlauf der Untersuchung ändernde Beziehung der Untersuchungsstichproben im Vergleich zur Normstichprobe deutlich. 


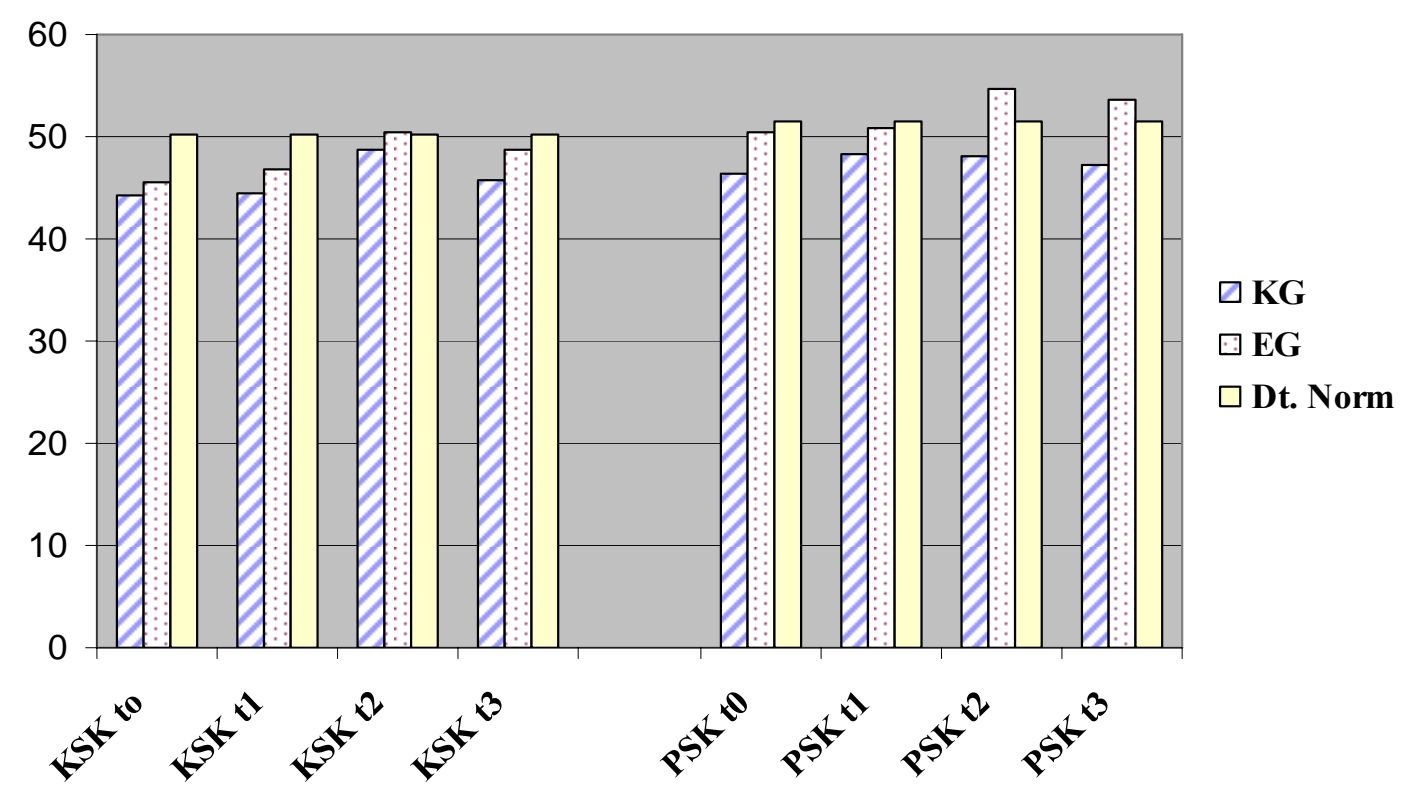

Abbildung 15: Veränderungen der körperlichen und psychischen Summenskalen der Experimentalgruppe $(n=30)$ und Kontrollgruppe $(n=28)$ über 4 Messzeitpunkte im Vergleich mit der deutschen Normstichprobe

Die Ausgangswerte der körperlichen Summenskala in der Baseline liegen deutlich unter denen der deutschen Normwerte. Sie steigen in der Wartezeit leicht an. Diese Veränderungen sind jedoch statistisch nicht signifikant. In der Treatmentphase ist ein Anstieg der Summenskalenwerte beider Gruppen und somit eine signifikante Verbesserung zu sehen. Während der Wert der Experimentalgruppe den Normwert übertrifft, bleibt der Wert der Kontrollgruppe trotz des Anstiegs darunter.

In der Follow-up-Periode fallen die Summenskalenscores beider Untersuchungsgruppen ab. Dieser Effekt ist bei der Kontrollgruppe größer als bei der Versuchsgruppe, jedoch nicht signifikant. Die Werte beider Gruppen sind bei Ende des Katamnesezeitraums signifikant höher als die Ausgangswerte vor Behandlungsbeginn.

Auch die Ausgangswerte der psychischen Summenskala liegen in der Baseline unter denen der deutschen Referenzgruppe. Die bzgl. der Untersuchungsgruppen beobachteten Veränderungen in der Wartezeit sind statistisch nicht signifikant (siehe Tabelle 25). Über die Treatmentzeit findet eine deutliche Verbesserung der psychischen Summenskalen der Experimentalgruppe statt. Der Wert bei Behandlungsende steigt über den der deutschen Normstichprobe hinaus. Bei der Kontrollgruppe zeigen sich kaum Veränderungen und die Werte des psychischen Wohlbefindens bleiben 
deutlich unter denen der deutschen Norm. Statistisch wird dieses in einem signifikanten Gruppenunterschied deutlich.

Obwohl in der Follow-up-Zeit ein leichtes Absinken der psychischen Summenskalenwerte beider Gruppen zu sehen ist, bleibt der Wert der Experimentalgruppe höher als der der deutschen Referenzgruppe. Die Werte der Kontrollgruppe liegen signifikant höher als der Ausgangswert bei Studienbeginn.

Die folgende Tabelle fasst die signifikanten Ergebnisse der varianzanalytischen Testung zusammen:

Tabelle 25: Signifikante Ergebnisse der zweifaktoriellen Varianzanalyse mit Messwiederholungen für die körperlichen und psychischen Summenskalenwerte über 4 Messzeitpunkte

\begin{tabular}{|l|l|l|l|}
\hline AV & Haupteffekt : Zeit & Signifikanz & Effektstärke $\left(\mathrm{Eta}^{2}\right)$ \\
\hline Körperliche Summensk. (t1-t2) & $\mathrm{F}=15,44$ & $\mathrm{p}=.000$ & $\eta^{2}=.219$ \\
\hline & Haupteffekt : Zeit & & \\
\hline Körperliche Summensk. (t2-t3) & $\mathrm{F}=5,81$ & $\mathrm{p}=.019$ & $\eta^{2}=.094$ \\
\hline & Haupteffekt : Zeit & & \\
\hline Körperliche Summensk. (t0-t3) & $\mathrm{F}=4,66$ & $\mathrm{p}=.035$ & $\eta^{2}=.078$ \\
\hline AV & Haupteffekt : Gruppe & Signifikanz & \\
\hline Psychische Summensk. (t1-t2) & $\mathrm{F}=4,32$ & $\mathrm{p}=.042$ & $\eta^{2}=.073$ \\
\hline & Haupteffekt : Gruppe & & \\
\hline Psychische Summensk. (t2-t3) & $\mathrm{F}=10,79$ & $\mathrm{p}=.002$ & $\eta^{2}=.161$ \\
\hline
\end{tabular}

\subsection{Erfassung des Körperkonzepts anhand der Frankfurter Körper- konzeptskalen (FKKS)}

Das Körperkonzept wird anhand der 9 Skalen des Frankfurter Fragebogens (FKKS) erfasst. Höhere Werte entsprechen einem generell besseren Körperkonzept.

Die folgende Tabelle zeigt die Veränderung der Mittelwerte der neun Skalen sowie des gesamten Summenscores (gebildet aus der Summe der neun Skalen) der Frankfurter Körperkonzeptskalen über den gesamten Studienverlauf. 
Tabelle 26: Ergebnisse der Frankfurter Körperkonzeptskalen (FKKS) der beiden Gruppen zu den vier Messzeitpunkten, $t 0=$ bei Studienbeginn, $t 1=4$ Wochen nach Kursbeginn, $t 2=8$ Wochen nach Kursbeginn (Kursende), $t 3=6$ Monate nach Kursende

\begin{tabular}{|c|c|c|c|c|}
\hline Experimentalgruppe $(\mathbf{n}=\mathbf{3 0})$ & to & t1 & t2 & t3 \\
\hline $\begin{array}{l}\text { SGKB: Skala, Gesundheit und } \\
\text { körperliches Befinden } \\
\text { Arithmetisches Mittel } \\
\text { Standardabweichung (SD) }\end{array}$ & $\begin{array}{c}21,7 \\
\pm 6,22\end{array}$ & $\begin{array}{c}22,4 \\
\pm 5,16 \\
\end{array}$ & $\begin{array}{l}24,87 \\
\pm 4,14\end{array}$ & $\begin{array}{c}24,83 \\
\pm 5,38\end{array}$ \\
\hline $\begin{array}{l}\text { SPKF: Skala, Pflege des Körpers } \\
\text { und der äußeren Erscheinung, } \\
\text { Beachtung der Funktionsfähigkeit } \\
\text { Arithmetisches Mittel } \\
\text { Standardabweichung (SD) }\end{array}$ & $\begin{array}{c}34,8 \\
\pm 4,78 \\
\end{array}$ & $\begin{array}{r}35,40 \\
\pm 4,65\end{array}$ & $\begin{array}{r}36,03 \\
\pm 3,94 \\
\end{array}$ & $\begin{array}{c}36,23 \\
\pm 4,48\end{array}$ \\
\hline $\begin{array}{l}\text { SKEF: Skala, körperliche } \\
\text { Effizienz } \\
\text { Arithmetisches Mittel } \\
\text { Standardabweichung (SD) }\end{array}$ & $\begin{array}{c}35,4 \\
\pm 7,93\end{array}$ & $\begin{array}{c}33,0 \\
\pm 7,63\end{array}$ & $\begin{array}{l}39,27 \\
\pm 7,54\end{array}$ & $\begin{array}{c}38,4 \\
\pm 8,71\end{array}$ \\
\hline $\begin{array}{c}\text { SKKO: Skala, Körperkontakt } \\
\text { Arithmetisches Mittel } \\
\text { Standardabweichung (SD) }\end{array}$ & $\begin{array}{l}22,43 \\
\pm 4,7 \\
\end{array}$ & $\begin{array}{r}22,33 \\
\pm 5,83 \\
\end{array}$ & $\begin{array}{c}24,57 \\
\pm 5,44 \\
\end{array}$ & $\begin{array}{r}25,37 \\
\pm 4,89 \\
\end{array}$ \\
\hline $\begin{array}{l}\text { SSEX: Skala, Sexualität } \\
\text { Arithmetisches Mittel } \\
\text { Standardabweichung (SD) }\end{array}$ & $\begin{array}{l}23,13 \\
\pm 4,00\end{array}$ & $\begin{array}{c}23,33 \\
\pm 4,23 \\
\end{array}$ & $\begin{array}{c}24,90 \\
\pm 3,80 \\
\end{array}$ & $\begin{array}{c}25,37 \\
\pm 3,76\end{array}$ \\
\hline $\begin{array}{l}\text { SSAK: Skala, Selbstakzeptanz } \\
\text { des Körpers } \\
\text { Arithmetisches Mittel } \\
\text { Standardabweichung (SD) }\end{array}$ & $\begin{array}{l}17,37 \\
\pm 5,71\end{array}$ & $\begin{array}{c}18,53 \\
\pm 5,54\end{array}$ & $\begin{array}{r}19,97 \\
\pm 5,29\end{array}$ & $\begin{array}{l}20,47 \\
\pm 5,20\end{array}$ \\
\hline $\begin{array}{l}\text { SAKA: Skala, Akzeptanz des } \\
\text { Körpers durch andere } \\
\text { Arithmetisches Mittel } \\
\text { Standardabweichung (SD) } \\
\end{array}$ & $\begin{array}{r}12,63 \\
\pm 3,55 \\
\end{array}$ & $\begin{array}{r}12,37 \\
\pm 3,90 \\
\end{array}$ & $\begin{array}{r}13,23 \\
\pm 3,82 \\
\end{array}$ & $\begin{array}{r}13,70 \\
\pm 4,02 \\
\end{array}$ \\
\hline $\begin{array}{l}\text { SASE: Skala, Aspekte der } \\
\text { körperlichen Erscheinung } \\
\text { Arithmetisches Mittel } \\
\text { Standardabweichung (SD) }\end{array}$ & $\begin{array}{c}58,6 \\
\pm 5,69 \\
\end{array}$ & $\begin{array}{r}58,47 \\
\pm 5,49\end{array}$ & $\begin{array}{c}59,7 \\
\pm 6,99\end{array}$ & $\begin{array}{l}59,17 \\
\pm 6,98\end{array}$ \\
\hline $\begin{array}{l}\text { SDIS: Skala, Dissimilatorische } \\
\text { Prozesse } \\
\text { Arithmetisches Mittel } \\
\text { Standardabweichung (SD) }\end{array}$ & $\begin{array}{l}16,77 \\
\pm 3,17\end{array}$ & $\begin{array}{l}17,43 \\
\pm 2,94 \\
\end{array}$ & $\begin{array}{l}17,70 \\
\pm 2,83\end{array}$ & $\begin{array}{r}17,43 \\
\pm 2,94 \\
\end{array}$ \\
\hline $\begin{array}{l}\text { Summenwert } \\
\text { Arithmetisches Mittel } \\
\text { Standardabweichung (SD) }\end{array}$ & $\begin{array}{l}242,83 \\
\pm 27,38\end{array}$ & $\begin{array}{l}243,27 \\
\pm 28,42\end{array}$ & $\begin{array}{l}260,23 \\
\pm 28,58\end{array}$ & $\begin{array}{l}260,97 \\
\pm 33,24\end{array}$ \\
\hline & to & t1 & t2 & t3 \\
\hline \multicolumn{5}{|l|}{ Kontrollgruppe (n=28) } \\
\hline $\begin{array}{l}\text { SGKB: Skala, Gesundheit und } \\
\text { körperliches Befinden } \\
\text { Arithmetisches Mittel } \\
\text { Standardabweichung (SD) }\end{array}$ & $\begin{array}{r}19,29 \\
\pm 5,73\end{array}$ & $\begin{array}{l}21 \\
\pm 5,17\end{array}$ & $\begin{array}{l}21,86 \\
\pm 5,63\end{array}$ & $\begin{array}{l}21,61 \\
\pm 6,46\end{array}$ \\
\hline $\begin{array}{l}\text { SPKF: Skala, Pflege des Körpers } \\
\text { und der äußeren Erscheinung, } \\
\text { Beachtung der Funktionsfähigkeit } \\
\text { Arithmetisches Mittel } \\
\text { Standardabweichung (SD) }\end{array}$ & $\begin{array}{c}34,14 \\
\pm 4,19\end{array}$ & $\begin{array}{r}33,93 \\
\pm 4,39\end{array}$ & $\begin{array}{c}34,0 \\
\pm 4,55\end{array}$ & $\begin{array}{c}33,29 \\
\pm 4,48\end{array}$ \\
\hline $\begin{array}{l}\text { SKEF: Skala, körperliche } \\
\text { Effizienz }\end{array}$ & 33,46 & 32,0 & 36,43 & 35,07 \\
\hline
\end{tabular}




\begin{tabular}{|c|c|c|c|c|}
\hline $\begin{array}{c}\text { Arithmetisches Mittel } \\
\text { Standardabweichung (SD) }\end{array}$ & $\pm 7,73$ & $\pm 6,61$ & $\pm 8,04$ & $\pm 6,95$ \\
\hline $\begin{array}{c}\text { SKKO: Skala, Körperkontakt } \\
\text { Arithmetisches Mittel } \\
\text { Standardabweichung (SD) }\end{array}$ & $\begin{array}{c}23,29 \\
\pm 5,21 \\
\end{array}$ & $\begin{array}{c}23,44 \\
\pm 5,87 \\
\end{array}$ & $\begin{array}{c}23,71 \\
\pm 5,62 \\
\end{array}$ & $\begin{array}{c}24,46 \\
\pm 5,04 \\
\end{array}$ \\
\hline $\begin{array}{c}\text { SSEX: Skala, Sexualität } \\
\text { Arithmetisches Mittel } \\
\text { Standardabweichung (SD) }\end{array}$ & $\begin{array}{r}22,39 \\
\pm 3,97 \\
\end{array}$ & $\begin{array}{c}22,41 \\
\pm 3,83 \\
\end{array}$ & $\begin{array}{r}22,36 \\
\pm 3,89 \\
\end{array}$ & $\begin{array}{r}22,50 \\
\pm 4,89 \\
\end{array}$ \\
\hline $\begin{array}{l}\text { SSAK: Skala, Selbstakzeptanz } \\
\text { des Körpers } \\
\text { Arithmetisches Mittel } \\
\text { Standardabweichung (SD) } \\
\end{array}$ & $\begin{array}{r}17,39 \\
\pm 5,32 \\
\end{array}$ & $\begin{array}{r}18,44 \\
\pm 4,72 \\
\end{array}$ & $\begin{array}{c}18,82 \\
\pm 5,11 \\
\end{array}$ & $\begin{array}{r}18,68 \\
\pm 5,03 \\
\end{array}$ \\
\hline $\begin{array}{l}\text { SAKA: Skala, Akzeptanz des } \\
\text { Körpers durch andere } \\
\text { Arithmetisches Mittel } \\
\text { Standardabweichung (SD) } \\
\end{array}$ & $\begin{array}{r}11,43 \\
\pm 2,47\end{array}$ & $\begin{array}{r}11,85 \\
\pm 2,92 \\
\end{array}$ & $\begin{array}{c}12,5 \\
\pm 2,62\end{array}$ & $\begin{array}{c}12,71 \\
\pm 3,16\end{array}$ \\
\hline $\begin{array}{l}\text { SASE: Skala, Aspekte der } \\
\text { körperlichen Erscheinung } \\
\text { Arithmetisches Mittel } \\
\text { Standardabweichung (SD) }\end{array}$ & $\begin{array}{c}54,21 \\
\pm 6,31 \\
\end{array}$ & $\begin{array}{c}54,52 \\
\pm 5,77\end{array}$ & $\begin{array}{l}55,46 \\
\pm 6,24\end{array}$ & $\begin{array}{c}55,68 \\
\pm 6,54 \\
\end{array}$ \\
\hline $\begin{array}{l}\text { SDIS: Skala, Dissimilatorische } \\
\text { Prozesse } \\
\text { Arithmetisches Mittel } \\
\text { Standardabweichung (SD) }\end{array}$ & $\begin{array}{c}16,32 \\
\pm 2,44\end{array}$ & $\begin{array}{c}16,41 \\
\pm 2,48\end{array}$ & $\begin{array}{c}16,36 \\
\pm 2,26\end{array}$ & $\begin{array}{r}16,57 \\
\pm 2,47\end{array}$ \\
\hline $\begin{array}{l}\text { Summenwert } \\
\text { Arithmetisches Mittel } \\
\text { Standardabweichung (SD) } \\
\end{array}$ & $\begin{array}{r}231,93 \\
\pm 24,04 \\
\end{array}$ & $\begin{array}{c}234 \\
\pm 24,66 \\
\end{array}$ & $\begin{array}{r}241,50 \\
\pm 27,51 \\
\end{array}$ & $\begin{array}{r}240,57 \\
\pm 29,58 \\
\end{array}$ \\
\hline
\end{tabular}

In der folgenden graphischen Darstellung werden die Verläufe des Gesamtsummenwertes in Versuchs- und Kontrollgruppe deutlich.

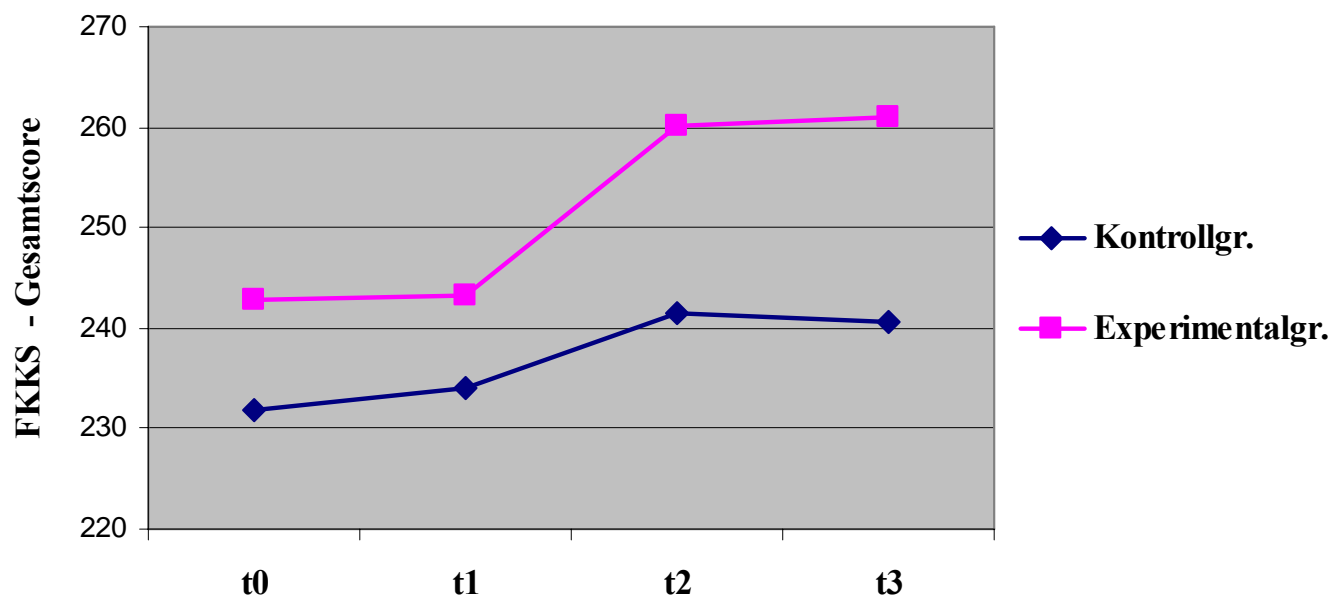

Abbildung 16: Veränderungen des Körperkonzepts (FKKS) der Experimentalgruppe $(n=30)$ und Kontrollgruppe $(n=28)$ über 4 Messzeitpunkte im Gruppenvergleich

In der Wartekontrollphase zeigen sich keine signifikanten Veränderungen bei beiden Gruppen bezüglich des Gesamtkörperkonzepts. Das Gesamtkörperkonzept verbessert sich während der Treatmentperiode für beide Gruppen hochsignifikant ( $F=43,50$; 
$\mathrm{p}=0,000)$. Der Anstieg bei den Teilnehmern der Experimentalgruppe ist jedoch höher. Hier zeichnet sich nach der zweifaktoriellen Varianzanalyse mit Messwiederholung eine Tendenz bezüglich des Faktors Gruppe $(F=3,66 ; p=0,061)$ sowie ein Gruppe*Zeit-Effekt $(\mathrm{F}=3,58 ; \mathrm{p}=0,064)$ ab.

In der Follow-up-Periode ist ein signifikanter Gruppenunterschied $(F=6,89$; $\mathrm{p}=0,011) \mathrm{zu}$ sehen. Während die Experimentalgruppe sich weiter leicht verbessert, gehen die Werte der Kontrollgruppe leicht zurück.

Die Auswertung der einzelnen Subskalen während der gesamten Studiendauer zeigt folgende signifikante Ergebnisse.

In der Baseline verbessern sich die Werte beider Gruppen bzgl. der Skala „Gesundheit und körperliches Befinden“ (SGKB) signifikant. Bzgl. der Skala „Körperliche Effizienz“ (SKEF) dagegen fallen die Werte bei beiden Gruppen zurück. In Bezug auf die Skala „Aspekte der körperlichen Erscheinung“ (SASE) zeigt sich ein signifikanter Gruppenunterschied.

Während des Treatments zeigen 7 von den insgesamt 9 Subskalen eine signifikante Veränderung für die Gesamtstichprobe.

Bzgl. der Skala „Gesundheit und körperliches Befinden“ (SGKB), Skala „Körperliche Effizienz“ (SKEF), Skala „Körperkontakt““(SKKO), Skala „Sexualität“ (SSEX), Skala „Selbstakzeptanz des Körpers“ (SSAK), Skala „Akzeptanz des Körpers durch andere“ (SAKA), Skala „Aspekte der körperlichen Erscheinung“ (SASE) verbessert sich die Gesamtstichprobe signifikant.

Die Kontrollgruppe für sich betrachtet zeigt keine Veränderungen bezüglich der Skalen „Sexualität“ (SSEX) und „Dissimilatorische Körperprozesse“ (SDIS). Hier gehen die Werte leicht zurück.

Die Subskala „Körperkontakt“ steigt bei der Kontrollgruppe signifikant (Interaktion) stärker als bei der Experimentalgruppe.

In der Katamnese zeichnen sich Veränderungen in der Skala „Gesundheit und körperliches Befinden“ (SGKB) ab. Diese Veränderungen sind bei der Experimentalgruppe signifikant positiver als bei der Kontrollgruppe.

Bei der Skala „Pflege des Körpers und der äußeren Erscheinung, Beachtung der Funktionsfähigkeit“ (SPKF) steigen die Werte der Experimentalgruppe, während die der Kontrollgruppe zurückfallen. 
Bei der Skala „Sexualität“ (SSEX) ist ein signifikant deutlicherer Anstieg in der Experimentalgruppe zu beobachten.

Bzgl. der Skala „Aspekte der körperlichen Erscheinung“ (SASE) sinkt der Wert in der Experimentalgruppe, der der Kontrollgruppe hingegen steigt. Auch hier handelt es sich um einen Gruppeneffekt.

Eine Tendenz bzgl. der „Körperlichen Effizienz“ (SKEF) zeichnet sich im Zeitraum der Nachuntersuchung für beide Gruppen ab, die Werte beider Gruppen fallen zurück.

Weiterhin findet sich die Tendenz eines Gruppenunterschiedes (siehe Tabelle 26) bezüglich der Skala „Dissimilatorische Körperprozesse“ (SDIS). Dieser ist durch einen leichten Rückfall bei der Experimentalgruppe bei leichtem Anstieg der Kontrollgruppe zu erklären.

Die folgende Tabelle fasst die signifikanten Ergebnisse der varianzanalytischen Testung zusammen:

Tabelle 27: Signifikante Ergebnisse der zweifaktoriellen Varianzanalyse mit Messwiederholungen für die FKKS Skalenwerte $(N=58)$

\begin{tabular}{|l|l|l|l|}
\hline AV & Haupteffekt : Zeit & Signifikanz & Eta $^{2}$ \\
\hline $\begin{array}{l}\text { SGKB: Skala, Gesundheit und } \\
\text { körperliches Befinden (t0-t1) }\end{array}$ & $\mathrm{F}=5,16$ & $\mathrm{p}=.027$ & $\eta^{2}=.086$ \\
\hline & Haupteffekt : Zeit & & \\
\hline $\begin{array}{l}\text { SKEF: Skala, körperliche Effizienz } \\
(\mathrm{t} 0-\mathrm{t} 1)\end{array}$ & $\mathrm{F}=14,38$ & $\mathrm{p}=.000$ & $\eta^{2}=.207$ \\
\hline & Haupteffekt : Gruppe & & \\
\hline $\begin{array}{l}\text { SASE: Skala, Aspekte der } \\
\text { körperlichen Erscheinung (t0-t1) }\end{array}$ & $\mathrm{F}=7,29$ & $\mathrm{p}=.009$ & $\eta^{2}=.117$ \\
\hline
\end{tabular}

\begin{tabular}{|l|l|l|l|}
\hline AV & Haupteffekt : Zeit & Signifikanz & Eta $^{2}$ \\
\hline $\begin{array}{l}\text { SGKB: Skala, Gesundheit und } \\
\text { körperliches Befinden (t1-t2) }\end{array}$ & $\mathrm{F}=15,22$ & $\mathrm{p}=.000$ & $\eta^{2}=.207$ \\
\hline $\begin{array}{l}\text { SKEF: Skala, körperliche Effizienz } \\
\text { (t1-t2) }\end{array}$ & $\mathrm{F}=90,2$ & $\mathrm{p}=.000$ & $\eta^{2}=.621$ \\
\hline AV & Haupteffekt : Zeit & Signifikanz & $\mathrm{Eta}^{2}$ \\
\hline SKKO: Skala, Körperkontakt (t1-t2) & $\mathrm{F}=12,72$ & $\mathrm{p}=.001$ & $\eta^{2}=.188$ \\
\hline & Interaktion: Gruppe* Zeit & & \\
\hline SKKO: Skala, Körperkontakt (t1-t2) & $\mathrm{F}=6,37$ & $\mathrm{p}=.008$ & $\eta^{2}=.119$ \\
\hline AV & Haupteffekt : Zeit & Signifikanz & Eta $^{2}$ \\
\hline SSEX: Skala, Sexualität (t1-t2) & $\mathrm{F}=4,29$ & $\mathrm{p}=.043$ & $\eta^{2}=.072$ \\
\hline & Haupteffekt: Gruppe & & \\
\hline SSEX: Skala, Sexualität (t1-t2) & $\mathrm{F}=3,00$ & $\begin{array}{l}\mathrm{p}=.09 \\
(\text { Tendenz) }\end{array}$ & $\eta^{2}=.052$ \\
\hline
\end{tabular}




\begin{tabular}{|l|l|l|l|}
\hline AV & Haupteffekt : Zeit & Signifikanz & $\mathrm{Eta}^{2}$ \\
\hline $\begin{array}{l}\text { SSAK: Skala, Selbstakzeptanz des } \\
\text { Körpers (t1-t2) }\end{array}$ & $\mathrm{F}=6,07$ & $\mathrm{p}=.012$ & $\eta^{2}=.109$ \\
\hline $\begin{array}{l}\text { SAKA: Skala, Akzeptanz des } \\
\text { Körpers durch andere (t1-t2) }\end{array}$ & $\mathrm{F}=9,66$ & $\mathrm{p}=.003$ & $\eta^{2}=.149$ \\
\hline AV & Haupteffekt : Zeit & Signifikanz & $\mathrm{Eta}^{2}$ \\
\hline $\begin{array}{l}\text { SASE: Skala, Aspekte der } \\
\text { körperlichen Erscheinung (t1-t2) }\end{array}$ & $\mathrm{F}=5,56$ & $\mathrm{p}=.021$ & $\eta^{2}=.093$ \\
\hline & Haupteffekt : Gruppe & & \\
\hline $\begin{array}{l}\text { SASE: Skala, Aspekte der } \\
\text { körperlichen Erscheinung (t1-t2) }\end{array}$ & $\mathrm{F}=6,52$ & $\mathrm{p}=.013$ & $\eta^{2}=.105$ \\
\hline AV & Haupteffekt : Gruppe & $\mathrm{p}=.056$ & $\eta^{2}=.065$ \\
\hline $\begin{array}{l}\text { SDIS: Skala, Dissimilatorische } \\
\text { Prozesse (t1-t2) }\end{array}$ & $\mathrm{F}=7,29$ & $\mathrm{Tendenz})$ & \\
\hline AV & Haupteffekt : Zeit & $\mathrm{p}=.000$ & $\eta^{2}=.442$ \\
\hline $\begin{array}{l}\text { FKKS GESAMT: Gesamt- } \\
\text { Körperkonzept (t1-t2) }\end{array}$ & $\mathrm{F}=43,50$ & $\begin{array}{l}\mathrm{p}=.061 \\
(\text { Tendenz) }\end{array}$ & $\eta^{2}=.062$ \\
\hline & Interaktion: Gruppe* Zeit & $\begin{array}{l}\mathrm{p}=.064 \\
(\text { Tendenz) }\end{array}$ & $\eta^{2}=.061$ \\
\hline & $\mathrm{F}=3,66$ & Haupteffekt : Gruppe &
\end{tabular}

\begin{tabular}{|c|c|c|c|}
\hline $\mathrm{AV}$ & Haupteffekt: Gruppe & Signifikanz & $\mathrm{Eta}^{2}$ \\
\hline $\begin{array}{l}\text { SGKB: Skala, Gesundheit und } \\
\text { körperliches Befinden (t2-t3) }\end{array}$ & $F=5,36$ & $p=.024$ & $\eta^{2}=.087$ \\
\hline $\begin{array}{l}\text { SPKF: Skala, Pflege des Körpers und } \\
\text { der äußeren Erscheinung, Beachtung } \\
\text { der Funktionsfähigkeit (t2-t3) }\end{array}$ & $\mathrm{F}=6,11$ & $p=.016$ & $\eta^{2}=.098$ \\
\hline SSEX: Skala, Sexualität (t2-t3) & $\mathrm{F}=7,71$ & $\mathrm{p}=.007$ & $\eta^{2}=.121$ \\
\hline $\begin{array}{l}\text { SASE: Skala, Aspekte der } \\
\text { körperlichen Erscheinung (t2-t3) }\end{array}$ & $F=5,35$ & $p=.024$ & $\eta^{2}=.087$ \\
\hline $\begin{array}{l}\text { SDIS: Skala, Dissimilatorische } \\
\text { Prozesse (t2-t3) }\end{array}$ & $\mathrm{F}=3,24$ & $\begin{array}{l}\mathrm{p}=.077 \\
\text { (Tendenz) }\end{array}$ & $\eta^{2}=.055$ \\
\hline $\mathrm{AV}$ & Haupteffekt : Zeit & & \\
\hline $\begin{array}{l}\text { SKEF: Skala, körperliche Effizienz } \\
\text { (t2-t3) }\end{array}$ & $\mathrm{F}=3,51$ & $\begin{array}{l}\mathrm{p}=.066 \\
\text { (Tendenz) }\end{array}$ & $\eta^{2}=.059$ \\
\hline AV & Haupteffekt : Gruppe & & \\
\hline $\begin{array}{l}\text { FKKS GESAMT: Gesamt- } \\
\text { Körperkonzept (t2-t3) }\end{array}$ & $\mathrm{F}=6,89$ & $\mathrm{p}=.011$ & $\eta^{2}=.110$ \\
\hline
\end{tabular}

Nach Deusinger (1988) können die Scores der Probanden und somit das Körperkonzept in die Bereiche negativ, neutral oder positiv eingeordnet werden.

In Abbildung 17 ist eine solche Kategorisierung des Körperkonzepts anhand der einzelnen Subskalen des FKKS getrennt nach Versuchs- und Kontrollgruppe zu den vier Messzeitpunkten dargestellt. 


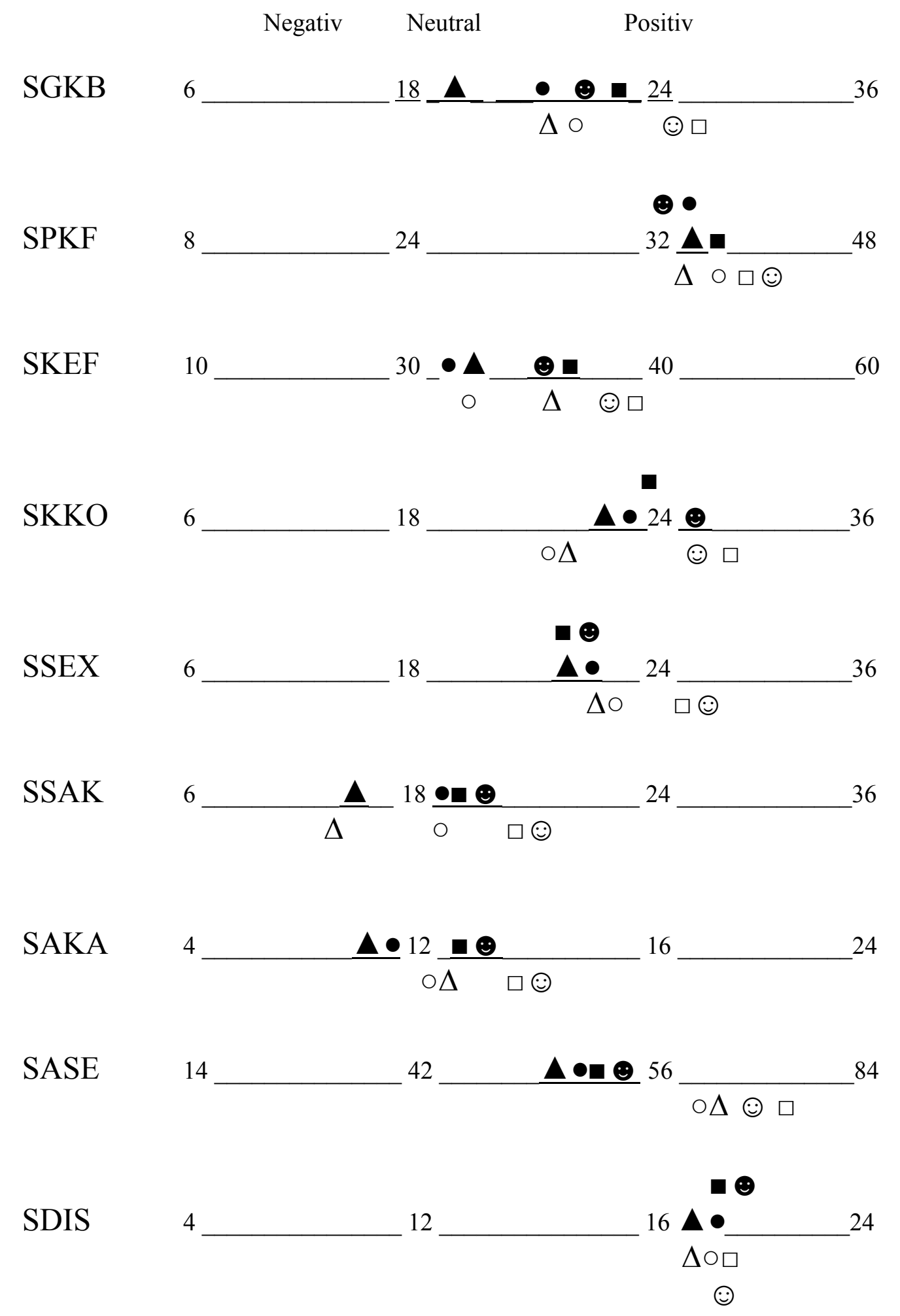

Abbildung 17: Ausrichtung des Körperkonzepts nach Deusinger auf den einzelnen Subskalen

\begin{tabular}{ccccc}
\hline & T0 & T1 & T2 & T3 \\
\hline KG & $\Delta$ & $\bullet$ & $\square$ & $\oplus$ \\
\hline EG & $\Delta$ & $\circ$ & $\square$ & $\odot$ \\
\hline
\end{tabular}




\subsection{Erfassung der Depressivität anhand des Beck-Depressions-Inventars (BDI)}

Die Summenwerte des BDI zeigen, dass die Ausprägung der depressiven Symptomatik für beide Gruppen zu allen Messzeitpunkten unauffällig bis mäßig, d.h. nicht pathologisch, ist.

Die folgende Tabelle zeigt den Verlauf der depressiven Symptomatik anhand der Summenwerte im BDI während der gesamten Studienzeit und den Summenwert der gesunden Norm (Hautzinger et al., 1995).

Es muss darauf hingewiesen werden, dass bei den berechneten Daten das 19. Item mitberechnet wurde (siehe Kap. 5.3.6).

Tabelle 28: Ergebnisse des Beck-Depressions-Inventars (BDI) der beiden Gruppen $z u$ den vier Messzeitpunkten, t0 = bei Aufnahme, $t 1=4$ Wochen nach Aufnahme (Kursbeginn), t2 $=8$ Wochen nach Kursbeginn (Kursende), $t 3=6$ Monate nach Kursende und einer gesunden Probandengruppe (Hautzinger et al., 1995)

\begin{tabular}{|c|c|c|c|c|}
\hline & $\mathbf{t 0}$ & $\mathbf{t 1}$ & $\mathbf{t 2}$ & $\mathbf{t 3}$ \\
\hline $\begin{array}{c}\text { Experimentalgruppe (n=30) } \\
\text { BDI Summenwert }\end{array}$ & & & & \\
Arithmetisches Mittel & 8,63 & 8,33 & 5,03 & 5,57 \\
Standardabweichung (SD) & $\pm 8,21$ & $\pm 8,31$ & $\pm 5,88$ & $\pm 5,99$ \\
\hline Kontrollgruppe (n=28) & & & & \\
BDI Summenwert & 10,96 & 11,54 & 9,71 & 10,00 \\
Arithmetisches Mittel & $\pm 6,78$ & $\pm 9,84$ & $\pm 11,18$ & $\pm 9,40$ \\
Standardabweichung (SD) & & & & \\
Gesunde Norm (n=86) & 6,45 & & & \\
BDI Summenwert & $\pm 5,2$ & & & \\
Arithmetisches Mittel & & & \\
Standardabweichung (SD) & &
\end{tabular}

Einteilung: $<11$ unauffällig, $11-17$ mäßige Ausprägung, $\geq 18$ klinisch relevant.

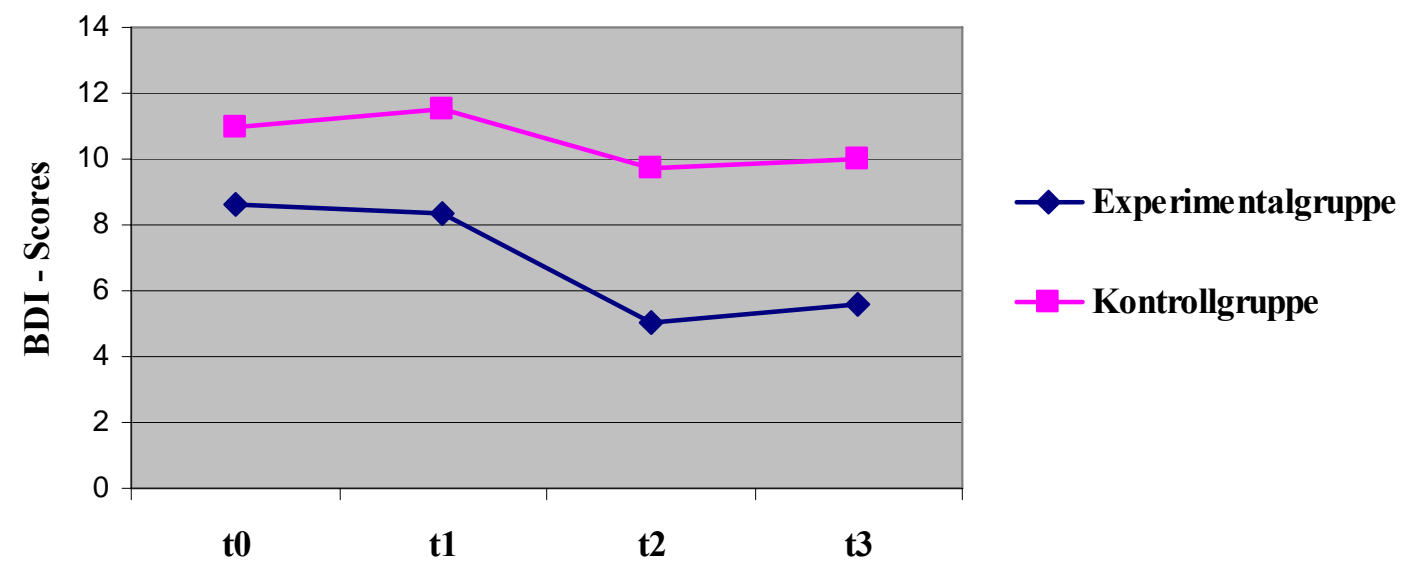

Abbildung 18: Veränderungen der depressiven Symptomatik (BDI) der Experimentalgruppe $(n=30)$ und Kontrollgruppe $(n=28)$ über 4 Messzeitpunkte im Gruppenvergleich 
In der Baseline finden sich bei keiner der beiden Gruppen signifikante Unterschiede, weder im Verlauf noch im Gruppenvergleich. Während der Treatmentperiode sinken die Werte, die die depressive Symptomatik ausdrücken, in beiden Gruppen signifikant (siehe Tabelle 29). Dieser Verlauf ist bzgl. der Experimentalgruppe signifikant stärker als bei der Kontrollgruppe. Varianzanalytische Berechnungen zeigen in Hinblick auf den Haupteffekt Gruppe eine Tendenz.

Der Haupteffekt Gruppe wird in der Katamnese signifikant. Signifikante Verlaufsveränderungen zeigen sich über den Zeitraum der Nachuntersuchung in beiden Gruppen nicht.

Die folgende Tabelle fasst die Ergebnisse der varianzanalytischen Testung zusammen:

Tabelle 29: Ergebnisse der zweifaktoriellen Varianzanalyse mit Messwiederholungen für den BDI über die Messzeitpunkte t1-t2/t2-t3, $(N=58)$

\begin{tabular}{|l|l|l|l|}
\hline AV & Haupteffekt : Zeit & Signifikanz & Effektstärke $\left(\mathrm{Eta}^{2}\right)$ \\
\hline $\begin{array}{l}\text { Depressive Symptomatik } \\
\text { (BDI) }(\mathrm{t} 1-\mathrm{t} 2)\end{array}$ & $\mathrm{F}=13,37$ & $\mathrm{p}=.001$ & $\eta^{2}=.193$ \\
\hline & Haupteffekt : Gruppe & & \\
\hline $\begin{array}{l}\text { Depressive Symptomatik } \\
\text { (BDI) ( } \mathrm{t} 1-\mathrm{t} 2)\end{array}$ & $\mathrm{F}=3,07$ & $\mathrm{p}=.085$ Tendenz & $\eta^{2}=.052$ \\
\hline & Haupteffekt : Gruppe & & \\
\hline $\begin{array}{l}\text { Depressive Symptomatik } \\
\text { (BDI) (t2-t3) }\end{array}$ & $\mathrm{F}=4,98$ & $\mathrm{p}=.03$ & $\eta^{2}=.082$ \\
\hline
\end{tabular}

Gemäß den im BDI-Handbuch (Hautzinger et al., 1995) vorgeschlagenen Kategorien können die beiden Gruppen, wie die folgende Tabelle zeigt, aufgeteilt werden:

Tabelle 30: Verlauf der BDI-Kategorien über die 4 Messzeitpunkte

\begin{tabular}{|l|c|c|c|}
\hline & $\begin{array}{c}\text { Unauffällig } \\
\text { BDI }>11\end{array}$ & $\begin{array}{c}\text { Mäßige } \\
\text { Ausprägung } \\
\text { BDI 11-17 }\end{array}$ & $\begin{array}{c}\text { Klinisch relevant } \\
\text { BDI } \geq 18\end{array}$ \\
\hline Experimentalgruppe to & $21(70 \%)$ & $6(20 \%)$ & $3(10 \%)$ \\
\hline Kontrollgruppe to & $15(55 \%)$ & $8(30 \%)$ & $4(15 \%)$ \\
\hline & & & \\
\hline Experimentalgruppe t1 & $22(73 \%)$ & $5(17 \%)$ & $3(10 \%)$ \\
\hline Kontrollgruppe t1 & $17(61 \%)$ & $6(21 \%)$ & $5(18 \%)$ \\
\hline & & & \\
\hline Experimentalgruppe t2 & $27(90 \%)$ & $2(7 \%)$ & $1(3 \%)$ \\
\hline Kontrollgruppe t2 & $21(75 \%)$ & $2(7 \%)$ & $5(18 \%)$ \\
\hline & & & \\
\hline Experimentalgruppe t3 & $25(83 \%)$ & $2(7 \%)$ & $3(10 \%)$ \\
\hline Kontrollgruppe t3 & $18(64 \%)$ & $2(7 \%)$ & $8(29 \%)$ \\
\hline
\end{tabular}


Im folgenden Balkendiagramm werden die Veränderungen der Verteilung auf die BDI- Kategorien im Verlauf der Untersuchung aufgezeigt.

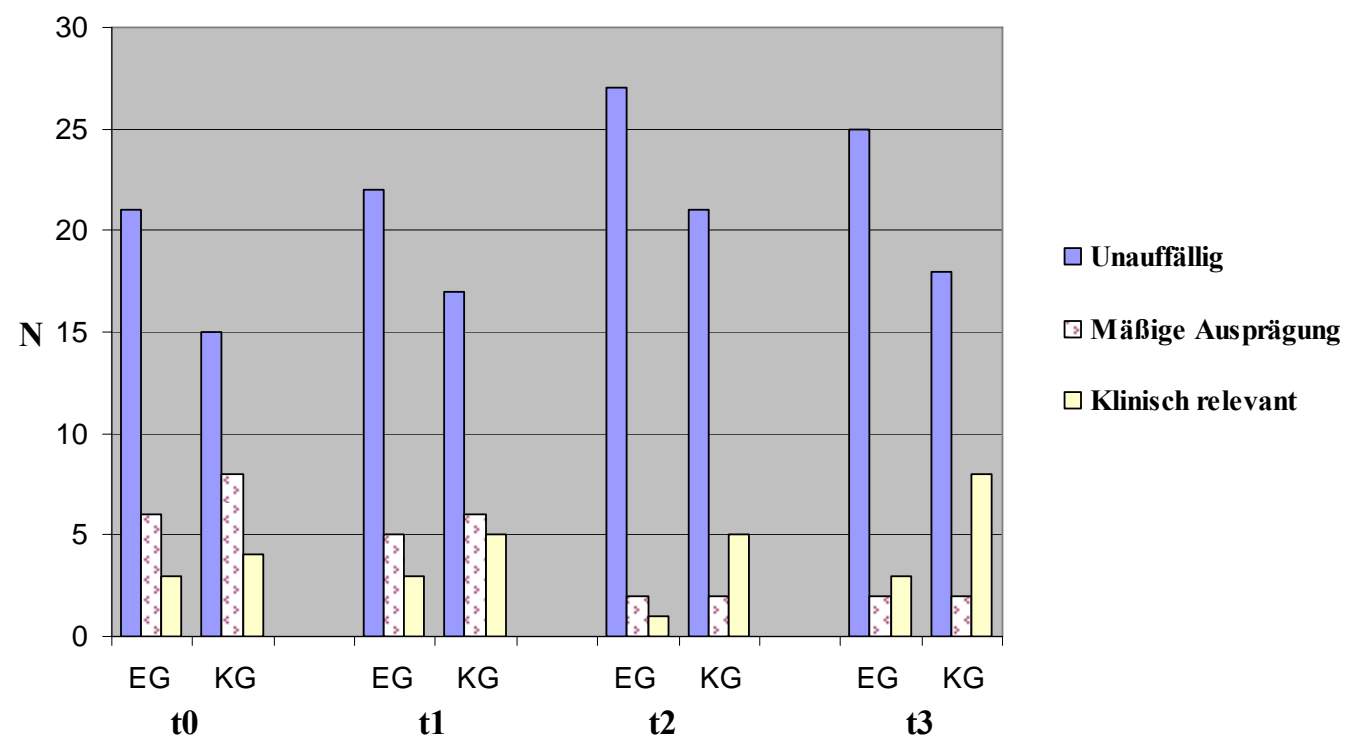

Abbildung 19: Verteilung auf die Kategorien im BDI der Experimentalgruppe $(n=30)$ und Kontrollgruppe $(\mathrm{n}=28)$ über 4 Messzeitpunkte im Gruppenvergleich

Die Interpretation der beobachteten Kategorienwechsel unterliegt der Einschränkung, dass im Grenzfall eine unbedeutende Veränderung um einen BDI-Punkt zu einem Kategorienwechsel führen kann. Aus diesem Grunde wurden auch keine Signifikanzprüfungen vorgenommen.

Es wird dennoch ersichtlich, dass die Mehrheit der Gesamtstichprobe (55-90\%) keine Depressivität aufweist. Der Prozentsatz der Teilnehmer mit einer unauffälligen Stimmungslage vergrößert sich während und nach den Bewegungsprogrammen. Der größere Anteil dieser Veränderung wird durch die Teilnehmer der Experimentalgruppe erklärt.

Die Anzahl der Teilnehmer mit einer mäßigen Ausprägung ist während der gesamten Studienzeit in beiden Gruppen vergleichbar. Auch hier nimmt die Anzahl während und nach den Interventionsprogrammen ab. Der Prozentsatz der Teilnehmer mit einer klinisch relevanten Depressivität variiert zwischen den beiden Gruppen insbesondere in der Treatmentperiode und zum Katamnesezeitpunkt. Bei der Experimentalgruppe handelt sich um höchstens 3 Teilnehmer (10\% der EG), welche sich nach dem Bewegungsbehandlungsprogramm auf 1 Fall (3 \% der EG) reduzieren. Im Vergleich dazu ist die Anzahl bei der Kontrollgruppe mit 5 Fällen (18 \% der KG) zu Beginn 
größer, bleibt aber nach dem Bewegungsgsprogramm unverändert. Sie steigt im Zeitraum der Katamnese auf 8 Fälle (29 \% der KG) an.

\subsection{Essstörungssymptomatik}

Durch die Anwendung des Strukturierten Inventars für Anorektische und Bulimische Essstörungen (SIAB) konnte das Vorkommen der Diagnose einer Binge Eating Disorder (BED) nach den DSM - IV Kriterien während der gesamten Studienzeit beobachtet werden.

Die Gesamtstichprobe beträgt für den SIAB $\mathrm{N}=57$, da eine Teilnehmerin der Experimentalguppe ohne Angabe von Gründen den SIAB nicht ausfüllte.

Zum Zeitpunkt t0 erfüllten insgesamt 7 Teilnehmer (12\% der gesamten Stichprobe) die Kriterien einer Binge Eating Störung. Bei Programmbeginn t1 waren es 5 (9\% der gesamten Stichprobe). Am Ende des Treatments und bei der abschließenden katamnestischen Untersuchung zeigten 4 Probanden ausschließlich Teilnehmer der Kontrollgruppe die volle Symptomatik der Binge Eating Störung (siehe Tabelle 31).

Der nichtparametrische Test (U-Test nach Mann und Whitney) zeigt, dass die Gruppenunterschiede während des Treatments und der Follow-up-Periode signifikant sind.

Tabelle 31: Teilnehmer der Kontroll- und Experimentalgruppe, die die Kriterien einer Binge Eating Disorder nach DSM - IV erfüllen über 4 Messzeitpunkte

\begin{tabular}{|l|c|c|c|c|}
\hline & T0 & T1 & T2 & T3 \\
\hline Experimentalgruppe (n=29) & 3 & 3 & - & - \\
\hline Kontrollgruppe (n=28) & 4 & 2 & 4 & 4 \\
\hline & n.s. & n.s. & $\mathrm{p}=, 033$ & $\mathrm{p}=, 033$ \\
\hline
\end{tabular}

\subsection{Wirkfaktoren des Behandlungsprogramms gemessen mit dem Dortmunder Fragebogen zur Bewegungstherapie (DFBT)}

Die Ergebnisse des DFBT zeigen, dass die Teilnehmer beider Gruppen die Wirksamkeit der verschiedenen Interventionen positiv bewerteten. Die Ratingskala des DFBT ist so gestaltet, dass bei Werten von 1-6 höhere Werte auf eine größere Wirksamkeit und Akzeptanz des Programms hinweisen. Die einzelnen Subskalen lassen sich wie folgt darstellen: 
Tabelle 32: Die 4 Skalen des DFBT mit ihren Mittelwerten, Standardabweichungen für die Versuchsgruppe und für die Kontrollgruppe zum Messzeitpunkt t2 (Ende des Treatments)

\begin{tabular}{|c|c|c|}
\hline & t2 & t2 \\
\hline AV & $\begin{array}{c}\text { Experimentalgruppe } \\
(n=29)\end{array}$ & $\begin{array}{c}\text { Kontrollgruppe } \\
(\mathrm{n}=\mathbf{2 8})\end{array}$ \\
\hline $\begin{array}{c}\text { DFBT- Biografische Erfahrungen } \\
\text { Arithmetisches Mittel } \\
\text { Standardabweichung (SD) }\end{array}$ & $\begin{array}{r}3,07 \\
\pm 1,2\end{array}$ & $\begin{array}{c}3,24 \\
\pm 1,24 \\
\end{array}$ \\
\hline $\begin{array}{l}\text { DFBT- Erfahrungen } \\
\text { von Körper und Selbst } \\
\text { Arithmetisches Mittel } \\
\text { Standardabweichung (SD) }\end{array}$ & $\begin{array}{c}4,87 \\
\pm 0,75\end{array}$ & $\begin{array}{c}4,49 \\
\pm 1\end{array}$ \\
\hline $\begin{array}{c}\text { DFBT- Erleben von Beziehungen } \\
\text { Arithmetisches Mittel } \\
\text { Standardabweichung (SD) }\end{array}$ & $\begin{array}{c}4,86 \\
\pm 0,63\end{array}$ & $\begin{array}{l}4,52 \\
\pm, 92\end{array}$ \\
\hline $\begin{array}{c}\text { DFBT- Erleben von } \\
\text { Bewegung und Wohlbefinden } \\
\text { Arithmetisches Mittel } \\
\text { Standardabweichung (SD) }\end{array}$ & $\begin{array}{l}5,85 \\
\pm 0,3\end{array}$ & $\begin{array}{r}5,49 \\
\pm, 54\end{array}$ \\
\hline
\end{tabular}

In der folgenden Abbildung werden die 4 DFBT-Subskalen im Gruppenvergleich graphisch dargestellt:

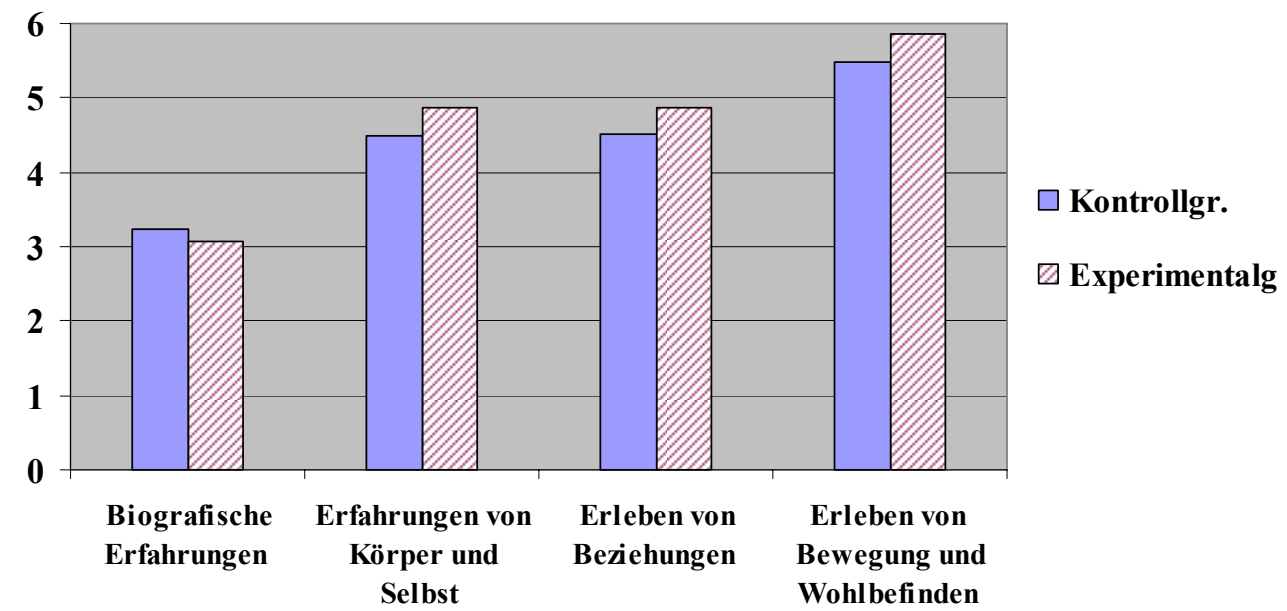

Abbildung 20: Die 4 Subskalen des Dortmunder Fragebogens (DFBT) der Experimentalgruppe $(n=29)$ und Kontrollgruppe $(n=28)$ zum Messzeitpunkt t2 im Gruppenvergleich

3 der insgesamt 4 Subskalen erreichen bei beiden Gruppen Werte zwischen 4,49 und 5,85 „Erfahrungen von Körper und Selbst“, „Erleben von Beziehungen“, „Erleben 
von Bewegung und Wohlbefinden" sind somit Subskalen, die als hilfreich erlebt werden. Im Gegensatz dazu steht die Subskala „Biografische Erfahrungen“. Sie erreicht mit 3,07 und 3,24 den niedrigsten Wert und wird im Mittelwert als „etwas zutreffend“ bis ,eher nicht zutreffend“ beurteilt.

Signifikante Gruppenunterschiede gibt es in der 4. Subskala „Erleben von Bewegung und Wohlbefinden". Sie wird von beiden Gruppen mit der positivsten Ratingkategorie „trifft sehr zu“ beurteilt, wobei die Einschätzung durch die Experimentalgruppe signifikant positiver ist als die der Kontrollgruppe.

Nach der einfaktoriellen ANOVA zeigt sich dieser signifikante Gruppenunterschied in der Subskala „Erleben von Bewegung und Wohlbefinden“ in folgender Ausprägung:

Tabelle 33: Subskala des DFBT „Erleben von Bewegung und Wohlbefinden“ im Gruppenvergleich bei Behandlungsende (t2)

\begin{tabular}{|l|c|c|c|}
\hline AV & & Signifikanz & Effektstärke $\left(\mathrm{Eta}^{2}\right)$ \\
\hline $\begin{array}{l}\text { DFBT- Erleben von Bewegung } \\
\text { und Wohlbefinden } \mathrm{t} 2\end{array}$ & $\mathrm{~F}=10,27$ & $\mathrm{p}=.002$ & $\eta^{2}=.219$ \\
\hline
\end{tabular}

\subsection{Die fahrradergometrische Belastungsuntersuchung}

Die fahrradergometrische Untersuchung zeigt bei einer Herzfrequenz von 130 Schlägen keine Gruppenunterschiede über die gesamt Studienzeit.

Beide Gruppen verbessern sich tendenziell in der Baselineperiode, dieser Werteanstieg ist statistisch gesehen nicht signifikant (siehe Tabelle 34).

Ein signifikanter Unterschied nach der zweifaktoriellen Varianzanalyse mit Messwiederholung (Haupteffekt Zeit) und somit deutliche Verbesserungen für beide Gruppen finden über die Zeit des Treatments statt. In der Follow-up-Periode bleiben die Werte und das erreichte Niveau bei der Kontrollgruppe konstant.

Im Vergleich dazu zeichnet sich bei der Experimentalgruppe ein Rückgang der aeroben Ausdauerleistung, gemessen bei der Pulsfrequenz 130, ab.

Nach varianzanalytischen Berechnungen (ANOVA) ist es eine Tendenz bzgl. der Interaktion Gruppe*Zeit.

Es macht Sinn, die durch die Fahrradergometrie erhaltenen Wattzahlen der Herzfrequenzen 130 auch als gewichtsbezogene Leistung (Watt/Körpergewicht kg) 
auszudrücken. Die Auswertung der absoluten und gewichtsbezogenen Leistungsfähigkeit ergibt keine relevanten Unterschiede und lässt sich wie folgt darstellen.

Tabelle 34: Mittelwerte und Standardabweichungen der absoluten (L) und der gewichtsbezogenen (L/Gew) Leistungsfähigkeit bei einer Herzfrequenz von 130 Schlägen über 4 Messzeitpunkte ( $t 0=$ bei Aufnahme, $t 1=4$ Wochen nach Aufnahme (Kursbeginn), $t 2=8$ Wochen nach Kursbeginn (Kursende), $t 3=6$ Monate nach Kursende); $(N=58)$

\begin{tabular}{|c|c|c|c|c|}
\hline & to & t1 & t2 & t3 \\
\hline Experimentalgruppe $(n=30)$ & \multirow[b]{2}{*}{$\begin{array}{c}91,59 \\
\pm 23,58\end{array}$} & \multirow[b]{2}{*}{$\begin{array}{c}96,82 \\
\pm 25,53 \\
\end{array}$} & \multirow[b]{2}{*}{$\begin{array}{r}104,87 \\
\pm 29,23\end{array}$} & \multirow[b]{2}{*}{$\begin{array}{c}97,94 \\
\pm 29,42 \\
\end{array}$} \\
\hline $\begin{array}{c}\text { L in Watt } \\
\text { Arithmetisches Mittel } \\
\text { Standardabweichung (SD) }\end{array}$ & & & & \\
\hline $\begin{array}{c}\mathrm{L} / \mathrm{Gew} \text { in Watt } / \mathrm{kg} \\
\text { Arithmetisches Mittel } \\
\text { Standardabweichung (SD) }\end{array}$ & $\begin{array}{l}, 86 \\
\pm, 23 \\
\end{array}$ & $\begin{array}{c}90 \\
\pm, 24\end{array}$ & $\begin{array}{r}1,00 \\
\pm, 29\end{array}$ & $\begin{array}{r}94 \\
\pm, 27\end{array}$ \\
\hline Kontrollgruppe (n=28) & \multirow[b]{2}{*}{$\begin{array}{c}90,47 \\
\pm 35,04 \\
\end{array}$} & \multirow[b]{2}{*}{$\begin{array}{c}91,78 \\
\pm 29,22 \\
\end{array}$} & \multirow[b]{2}{*}{$\begin{array}{r}100,50 \\
\pm 28,52 \\
\end{array}$} & \multirow[b]{2}{*}{$\begin{array}{r}100,19 \\
\pm 29,51 \\
\end{array}$} \\
\hline $\begin{array}{c}\text { L in Watt } \\
\text { Arithmetisches Mittel } \\
\text { Standardabweichung (SD) }\end{array}$ & & & & \\
\hline $\begin{array}{c}\mathrm{L} / \mathrm{Gew} \text { in Watt/kg } \\
\text { Arithmetisches Mittel } \\
\text { Standardabweichung (SD) }\end{array}$ & $\begin{array}{c}, 80 \\
\pm, 27\end{array}$ & $\begin{array}{c}, 83 \\
\pm, 22\end{array}$ & $\begin{array}{c}, 92 \\
\pm, 20\end{array}$ & $\begin{array}{c}92 \\
\pm, 23\end{array}$ \\
\hline
\end{tabular}

Die folgende Tabelle fasst die signifikanten Ergebnisse der varianzanalytischen Testung zusammen:

Tabelle 35: Die signifikanten Ergebnisse der zweifaktoriellen Varianzanalyse mit Messwiederholung auf dem Faktor Zeit ( $t 0=$ bei Aufnahme, $t 1=4$ Wochen nach Aufnahme (Kursbeginn), $t 2=8$ Wochen nach Kursbeginn (Kursende), $t 3=6$ Monate nach Kursende) für die absolute (L) und gewichtsbezogene (L/Gew) Leistungsfähigkeit bei einer Herzfrequenz von 130 Schlägen; $(N=58)$

\begin{tabular}{|l|l|l|l|}
\hline AV & Haupteffekt $:$ Zeit & Signifikanz & Effektstärke $\left(\right.$ Eta $\left.^{2}\right)$ \\
\hline L in Watt (t0-t1) & $\mathrm{F}=3,63$ & $\mathrm{p}=.062$ Tendenz & $\eta^{2}=.066$ \\
\hline L in Watt $/ \mathrm{kg}(\mathrm{t} 0-\mathrm{t} 1)$ & $\mathrm{F}=4,54$ & $\mathrm{p}=.038$ & $\eta^{2}=.082$ \\
\hline L in Watt (t1-t2) & $\mathrm{F}=14,71$ & $\mathrm{p}=.000$ & $\eta^{2}=.227$ \\
\hline L in Watt/kg (t1-t2) & $\mathrm{F}=21,48$ & $\mathrm{p}=.000$ & $\eta^{2}=.301$ \\
\hline & Interaktion: Gruppe* Zeit & & \\
\hline L in Watt (t2-t3) & $\mathrm{F}=3,17$ & $\mathrm{p}=.081$ Tendenz & $\eta^{2}=.062$ \\
\hline L in Watt/kg (t2-t3) & $\mathrm{F}=3,87$ & $\mathrm{p}=.081$ Tendenz & $\eta^{2}=.062$ \\
\hline
\end{tabular}


Die Entwicklung der absoluten Leistungsfähigkeit (L) wird in der graphischen Darstellung verdeutlicht.

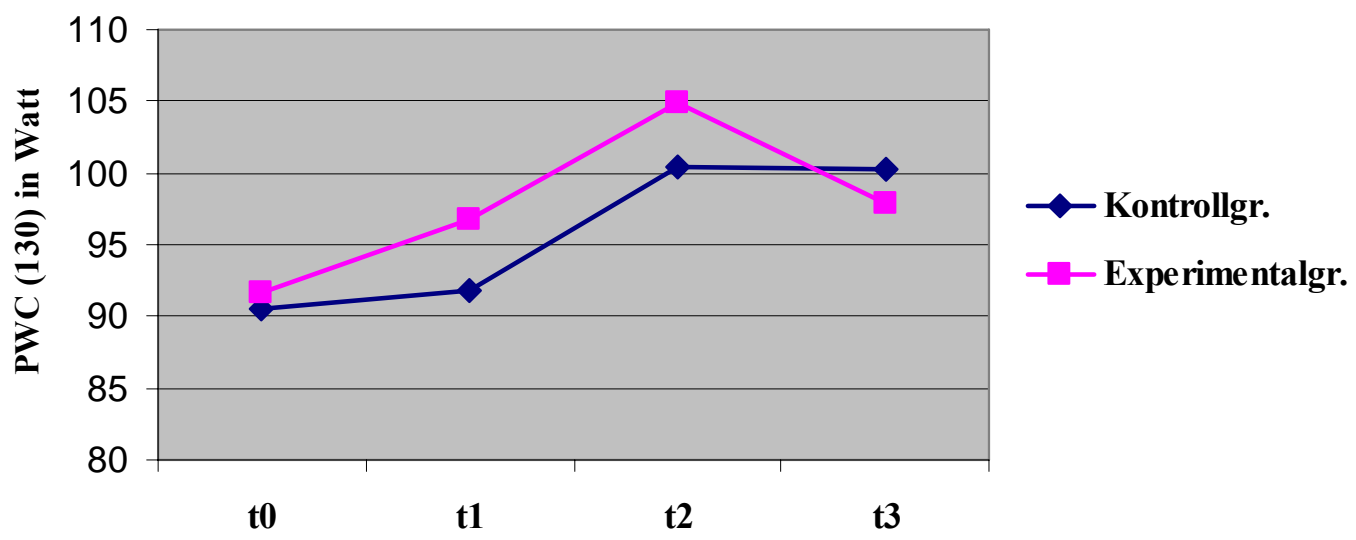

Abbildung 21: Veränderungen der absoluten Leistungsfähigkeit (L) bei einer Herzfrequenz von 130 Schlägen der Experimental- und Kontrollgruppe über 4 Messzeitpunkte im Gruppenvergleich; $(\mathrm{N}=58)$

Noch aussagekräftiger wird es, wenn die o.g. relative Leistung in ihrem Verhältnis zu den Normwerten dargestellt wird.

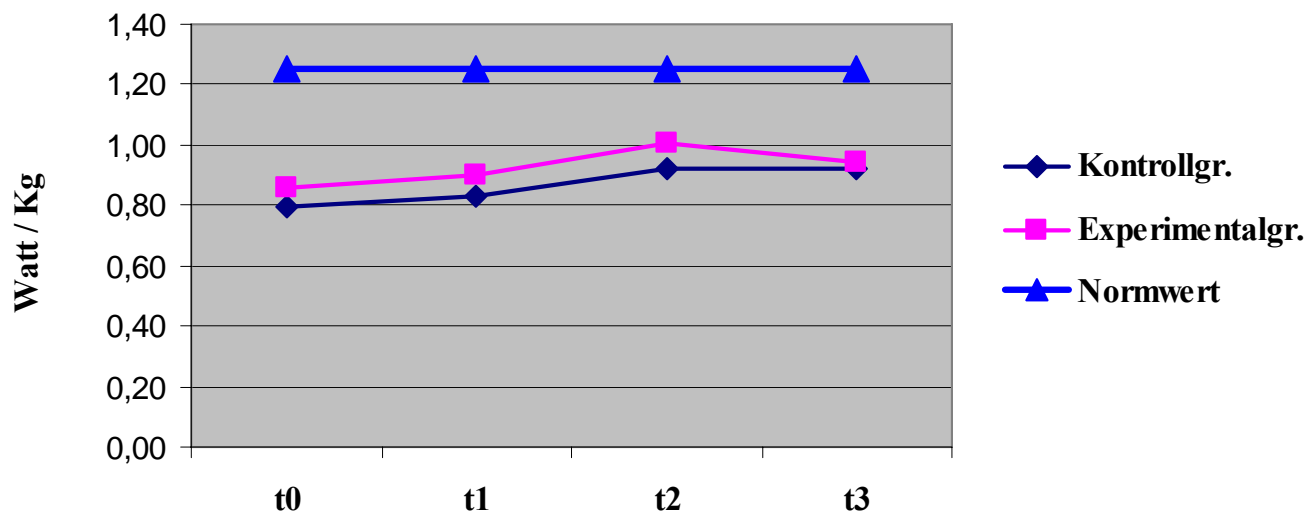

Abbildung 22: Veränderungen der gewichtsbezogenen Leistungsfähigkeit (L/Gew) bei einer Herzfrequenz von 130 Schlägen der Experimental- und Kontrollgruppe über 4 Messzeitpunkte im Gruppen- und Sollwertvergleich; $(\mathrm{N}=58)$

Der Vergleich der Gruppen mit den Normwerten zeigt, dass beide Gruppen trotz der Verbesserungen weiterhin unter der für das Geschlecht und Gewicht bestehenden Norm bleiben. 


\subsection{Der Walkingtest}

Durch die Berechnungsformel des Walkingtests wird ein Leistungsindex (vgl. Kapitel 5.3.10), der mathematische Grundlage der Interpretation des Walktests ist, ermittelt.

Die Berechnungsformel ist jedoch für stark übergewichtige Teilnehmer ungeeignet, da sie Minuswerte ergibt. Vier Teilnehmer, 3 aus der Kontrollgruppe und 1 aus der Experimentalgruppe, waren davon betroffen und werden aus diesem Grund bei der Gesamtauswertung nicht mitberücksichtigt. Es handelt sich um Teilnehmer, die ein extremes Übergewicht, Adipositas 3. Grades bei einem BMI von 44,7 - 57,2 aufwiesen.

Geringe weitere Ausfälle gab es zu verschiedenen Testzeitpunkten von Teilnehmern, die aus medizinischen Gründen den Test nicht absolviert konnten (siehe Tabelle 36).

Bei der Berechnung wurde nach der ITT Methode verfahren.

Tabelle 36: Mittelwerte und Standardabweichung des Walkingtest-Index der beiden Gruppen zu den vier Messzeitpunkten, t0 = bei Aufnahme, $t 1=4$ Wochen nach Aufnahme (Kursbeginn), $t 2=8$ Wochen nach Kursbeginn (Kursende), $t 3=6$ Monate nach Kursende

\begin{tabular}{|c|c|c|c|c|}
\hline & t0 & t1 & $\mathbf{t} 2$ & t3 \\
\hline $\begin{array}{c}\text { Experimentalgruppe }(\mathbf{n}=\mathbf{2 9}) \\
\text { Tatsächlicher Teilnehmer }\end{array}$ & 29 & 27 & 26 & 28 \\
\hline $\begin{array}{c}\text { Leistungsindex } \\
\text { Arithmetisches Mittel } \\
\text { Standardabweichung (SD) }\end{array}$ & $\begin{array}{c}55,20 \\
\pm 15,16\end{array}$ & $\begin{array}{c}61,49 \\
\pm 15,21\end{array}$ & $\begin{array}{c}70,48 \\
\pm 14,05\end{array}$ & $\begin{array}{r}67,29 \\
\pm 18,67\end{array}$ \\
\hline $\begin{array}{l}\text { Kontrollgruppe }(\mathbf{n}=\mathbf{2 5}) \\
\text { Tatsächlicher Teilnehmer }\end{array}$ & 25 & 25 & 24 & 25 \\
\hline $\begin{array}{c}\text { Leistungsindex } \\
\text { Arithmetisches Mittel } \\
\text { Standardabweichung (SD) }\end{array}$ & $\begin{array}{c}50,64 \\
\pm 12,95\end{array}$ & $\begin{array}{c}53,74 \\
\pm 14,32\end{array}$ & $\begin{array}{c}61,73 \\
\pm 14,94\end{array}$ & $\begin{array}{c}56,74 \\
\pm 17,35\end{array}$ \\
\hline
\end{tabular}

Die folgende Tabelle fasst die signifikanten Ergebnisse der varianzanalytischen Testung zusammen: 
Tabelle 37: Signifikante Ergebnisse der zweifaktoriellen Varianzanalyse mit Messwiederholungen auf dem Faktor Zeit (t0-t1; t1-t2, t2-t3) für den WalkingtestIndex

\begin{tabular}{|l|l|l|l|}
\hline AV & Haupteffekt : Zeit & Signifikanz & Effektstärke $\left(\mathrm{Eta}^{2}\right)$ \\
\hline Walkingtest-Index (t0-t1) & $\mathrm{F}=19,95$ & $\mathrm{p}=.000$ & $\eta^{2}=.276$ \\
\hline Walkingtest-Index (t1-t2) & $\mathrm{F}=37,48$ & $\mathrm{p}=.000$ & $\eta^{2}=.449$ \\
\hline & Haupteffekt : Gruppe & & \\
\hline Walkingtest-Index (t1-t2) & $\mathrm{F}=6,53$ & $\mathrm{p}=.014$ & $\eta^{2}=.124$ \\
\hline & Haupteffekt : Zeit & & \\
\hline Walkingtest-Index (t2-t3) & $\mathrm{F}=4,9$ & $\mathrm{p}=.032$ & $\eta^{2}=.095$ \\
\hline & Haupteffekt : Gruppe & & \\
\hline Walkingtest-Index (t2-t3) & $\mathrm{F}=4,7$ & $\mathrm{p}=.035$ & $\eta^{2}=.091$ \\
\hline
\end{tabular}

Der Vergleich der beiden Gruppen ergibt über die Gesamtstudienzeit signifikante Ergebnisse.

Während der Baselineperiode steigen die Werte beider Gruppen signifikant.

Der Werteanstieg setzt sich für beide Gruppe während der Behandlung fort. Hier ist ein signifikanter Effekt bzgl. der Zeit und auch der Gruppe zu sehen. Während der Follow-up-Periode zeichnet sich ein Rückgang für beide Gruppen ab, auch dieser ist bzgl. Zeit und Gruppe signifikant. Die Werte sind jedoch zum Testzeitpunkt t3 höher als zu den Testzeitpunkten vor dem Treatment.

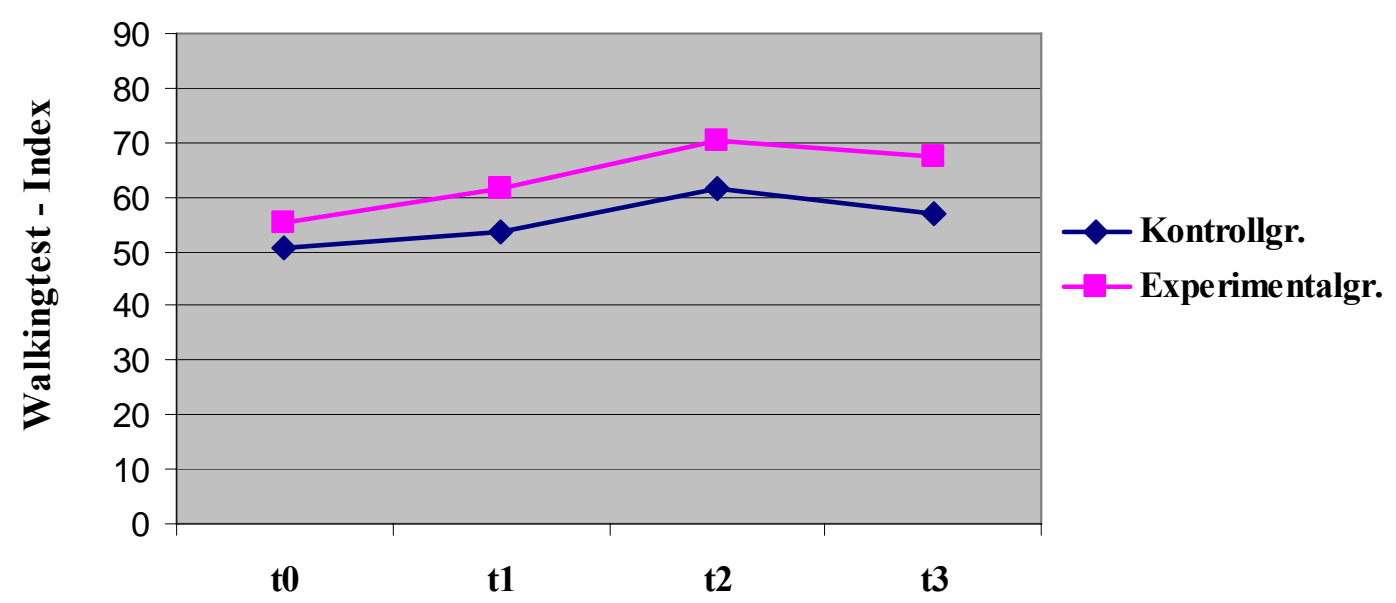

Abbildung 23: Verlauf des Walkingtest-Leistungsindex der Experimentalgruppe $(n=29)$ und Kontrollgruppe $(n=25)$ über 4 Messzeitpunkte im Gruppenvergleich 
Anhand einer fünfstufigen Leistungskategorisierung kann der Leistungsindex der Teilnehmer mit Normwerten verglichen werden (siehe Tabelle 38).

Die Tatsache, dass der höchste Wert für beide Gruppen während der ganzen Studie der der Experimentalgruppe am Ende des Treatments mit 70 ist, macht deutlich, dass die aerobe Leistungsfähigkeit nach diesen Klassifikationsmustern mit Ausnahme dieses einen Wertes als sehr schwach zu bezeichnen ist.

Tabelle 38: Klassifikation des Walkingtest-Index (WI) in 5 Stufen (Bös et al, 1992, 173)

\begin{tabular}{r|l}
\hline \multicolumn{1}{l|}{ WI } & \multicolumn{1}{c}{ Leistungskategorie } \\
\hline$>130$ & sehr gut \\
\hline $90-110$ & durchschnittlich \\
\hline $70-89$ & schwach \\
\hline$<70$ & sehr schwach \\
\hline
\end{tabular}

\subsection{Der Koordinationstest}

Der Koordinationstest (BKT-Kur) zeigt keine signifikanten Gruppenunterschiede zu keinem Messzeitpunkt der gesamten Untersuchung.

Beide Gruppen besitzen ein ähnliches koordinatives Ausgangsniveau und verbessern sich leicht während des Zeitraums der Baseline. Dies ist nach der varianzanalytischen Berechnung ein signifikanter Zeiteffekt.

In der Treatmentphase setzen sich die Verbesserungen der Koordinationsfähigkeit für beide Gruppen fort. Auch hier ist die Veränderung über die Zeit in beiden Gruppen signifikant.

In der Follow-up-Periode findet für die Experimentalgruppe eine leichte Verbesserung statt. In der Kontrollgruppe geht der Wert leicht zurück.

Dieser Gruppenunterschied ist nach varianzanalytischer Berechnung eine Tendenz (siehe Tabelle 39).

Die folgende Tabelle zeigt die Veränderungen der Mittelwerte des Koordinationstests BKT-Kur während der gesamten Studienzeit. 
Tabelle 39: Ergebnisse des BKT-Kur der beiden Gruppen zu den vier Messzeitpunkten, $t 0=$ bei Aufnahme, $t 1=4$ Wochen nach Aufnahme (Kursbeginn), t2 $=8$ Wochen nach Kursbeginn (Kursende), $t 3=6$ Monate nach Kursende

\begin{tabular}{|c|c|c|c|c|}
\hline & $\mathbf{t 0}$ & $\mathbf{t 1}$ & $\mathbf{t 2}$ & $\mathbf{t 3}$ \\
\hline $\begin{array}{c}\text { Experimentalgruppe (n=30) } \\
\text { Summenscore des BKT-Kur }\end{array}$ & 3,90 & 4,9 & 6,61 & 6,71 \\
$\begin{array}{c}\text { Arithmetisches Mittel } \\
\text { Standardabweichung (SD) }\end{array}$ & $\pm 1,39$ & $\pm 1,7$ & $\pm 1,57$ & $\pm 1,82$ \\
\hline Kontrollgruppe (n=28) & & & & \\
Summenscore des BKT-Kur & 3,61 & 4,37 & 5,74 & 5,54 \\
Arithmetisches Mittel & $\pm 1,95$ & $\pm 1,9$ & $\pm 2,12$ & $\pm 2,04$ \\
Standardabweichung (SD) & & &
\end{tabular}

In der folgenden Abbildung ist der Verlauf der Ergebnisse des Koordinationstests BKT-Kur graphisch dargestellt

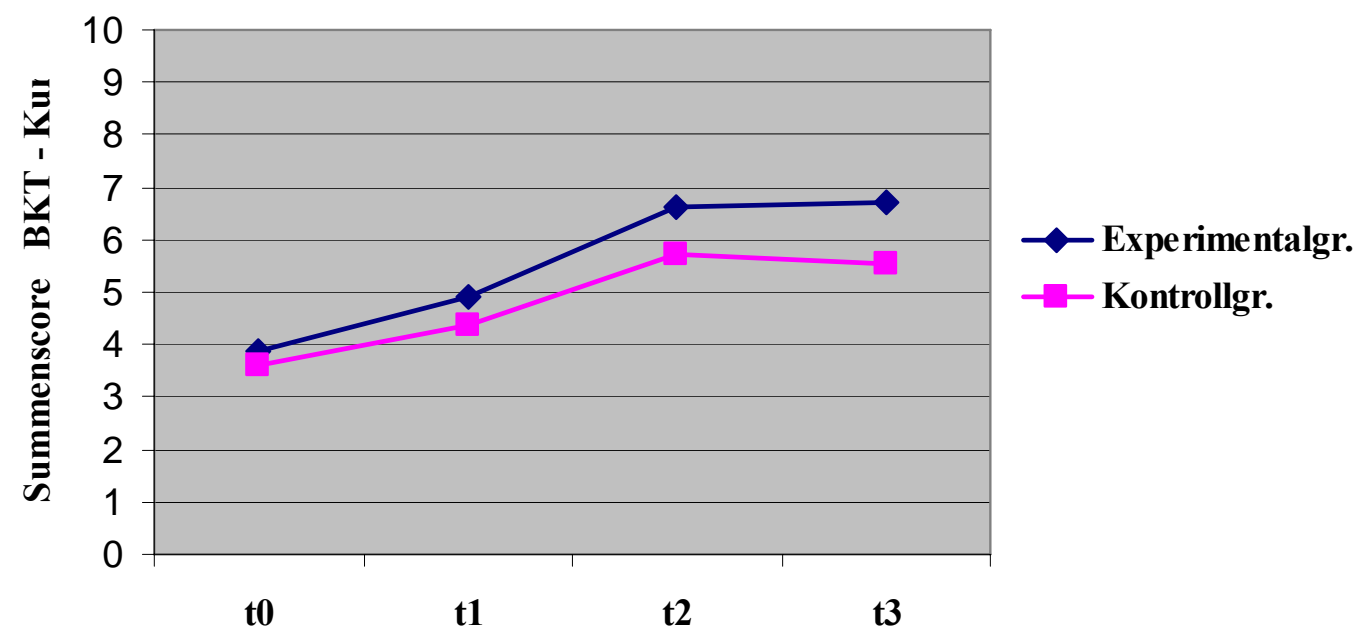

Abbildung 24: Verlauf der Koordinationsfähigkeit nach dem BKT-Kur der Experimentalgruppe $(n=30)$ und Kontrollgruppe $(n=28)$ über 4 Messzeitpunkte im Gruppenvergleich

Die folgende Tabelle zeigt die Ergebnisse der varianzanalytischen Testung:

Tabelle 40: Signifikante Ergebnisse der zweifaktoriellen Varianzanalyse mit Messwiederholungen der Koordinationsfähigkeit nach dem BKT-Kur über 4 Messzeitpunkte

\begin{tabular}{|l|l|l|l|}
\hline AV & Haupteffekt $:$ Zeit & Signifikanz & Effektstärke $\left(\mathrm{Eta}^{2}\right)$ \\
\hline BKT - Kur (t0-t1) & $\mathrm{F}=17,42$ & $\mathrm{p}=.000$ & $\eta^{2}=.244$ \\
\hline BKT - Kur (t1-t2) & $\mathrm{F}=62,64$ & $\mathrm{p}=.000$ & $\eta^{2}=.546$ \\
\hline & Haupteffekt $:$ Gruppe & & \\
\hline BKT - Kur (t2-t3) & $\mathrm{F}=3,65$ & $\mathrm{p}=.062$ Tendenz & $\eta^{2}=.067$ \\
\hline
\end{tabular}


Für die Interpretation der Testergebnisse können zusätzlich die Normwerte für Männer und Frauen der verschiedenen Altersgruppierungen herangezogen werden. Das Durchschnittsalter für die Gesamtstichprobe beträgt 44 Jahre.

Der Vergleich der Mittelwerte der koordinativen Leistungsfähigkeit der Studienstichprobe mit den alters- und geschlechtsabhängigen Normwerten zeigt, dass die Teilnehmer der Studie sich von den Kategorien „sehr schwach“ und „schwach“ (gemessen vor dem Programm) zu der Kategorie „mittel“ entwickeln (Ende des Programms und Katamnese).

Die folgende Tabelle stellt die Normwerte für Männer und Frauen verschiedener Altersgruppierungen dar.

Tabelle 41: Normwerte für den Bewegungskoordinationstest, $N=1831$, (Bös et al., 1992, 160)

\begin{tabular}{|lc|cccc|}
\hline Summenscore & & \multicolumn{4}{|c|}{ Altersgruppen (Jahre) } \\
beim BKT- Kur & & $\mathbf{2 0} \mathbf{- 2 9}$ & $\mathbf{3 0} \mathbf{- 3 9}$ & $\mathbf{4 0} \mathbf{- 4 9}$ & $\mathbf{5 0}$ - 59 \\
\hline \multirow{2}{*}{ sehr gut } & Männer & $>8$ & $>8$ & $>8$ & $>7$ \\
& Frauen & $>8$ & $>8$ & $>7$ & $>6$ \\
gut & Männer & 8 & 8 & 8 & 7 \\
& Frauen & 8 & 8 & 7 & 6 \\
mittel & Männer & $6-7$ & $6-7$ & $6-7$ & $5-6$ \\
& Frauen & $6-7$ & $6-7$ & $5-6$ & $4-5$ \\
schwach & Männer & 6 & 6 & 6 & 5 \\
sehr & Frauen & 6 & 6 & 5 & 4 \\
schwach & Männer & $<5$ & $<5$ & $<5$ & $<4$ \\
& Frauen & $<5$ & $<5$ & $<4$ & $<3$ \\
\hline
\end{tabular}

\subsection{Der Krafttest}

Es wurde ein Krafttest mit 3 Aufgaben, „Sit-ups“, „Liegestütze“ und „Jump and Reachs“ durchgeführt. Der Vergleich der beiden Gruppen bezüglich des Krafttests ergibt keine signifikanten Gruppenunterschiede.

Eine Teilnehmerin der Kontrollgruppe (Alter 47 Jahre, BMI=50,1) konnte aufgrund ihrer allgemeinen körperlichen Verfassung an keinem der Krafttests teilnehmen.

Die Ergebnisse werden anhand der 3 Kraftaufgaben getrennt dargestellt.

Die Durchführung der Aufgabe „Sit-ups“ war für eine Teilnehmerin der Experimentalgruppe (Alter 69 Jahre, BMI=37,09) aus medizinischen Gründen nicht 
möglich, und sie wurde somit von der Auswertung ausgeschlossen. Weiterhin war es für drei Teilnehmer der Experimentalgruppe und vier der Kontrollgruppe aus Kraftmangel und/oder wegen aktueller körperlicher Beschwerden zu verschiedenen Messzeitpunkten nicht möglich, wiederholt „Sit-ups“ auszuführen. Die Versuche dieser Teilnehmer sind mit 0 Punkten berechnet worden.

Die Anzahl der wiederholten „Sit-ups“ in der Baseline Periode bleibt in der Experimentalgruppe relativ konstant. In der Kontrollgruppe steigt die Anzahl der „Sit-ups“ deutlich an. Dies ist nach der varianzanalytischen Berechnung sowohl ein signifikanter Haupteffekt bzgl. der Zeit wie auch eine Interaktion Zeit*Gruppe. Während der Treatmentperiode verbessern sich beide Gruppen deutlich. Der Anstieg der Experimentalgruppe ist wesentlich höher als der der Kontrollgruppe, so dass der Wert der Experimentalgruppe den der Kontrollgruppe zu Messzeitpunkt t2 übertrifft. Diese Veränderung wird nach der varianzanalytischen Berechnung in der Interaktion Zeit*Gruppe als hoch signifikant interpretiert.

Gleichzeitig ist die Verbesserung beider Gruppen bzgl. des Faktors Zeit auch hier sehr signifikant. In der Follow-up-Periode bleiben die Anzahl der „Sit-ups“ für beide Gruppen fast unverändert. Es bestehen keinerlei Signifikanzen.

Das Ausgangsniveau der beiden Gruppen bezüglich der „Liegestütze“ ist leicht unterschiedlich aber vergleichbar und statistisch nicht signifikant. Die Anzahl der Liegestütze während der zwei Messzeitpunkte der Baseline Periode erhöht sich leicht, jedoch für beide Gruppen in vergleichbarem Ausmaß. Diese leichte Verbesserung ist nach der varianzanalytischen Berechnung signifikant im Bezug auf den Faktor Zeit.

In der Treatmentperiode setzt sich der Werteanstieg für beiden Gruppen deutlich fort. Diese Verbesserung ist für die Experimentalgruppe größer als für die Kontrollgruppe.

Der Gruppenunterschied der Baselinephase wird aufgeholt, und so kommt es $\mathrm{zu}$ einem Werteausgleich zwischen den zwei Gruppen zum Ende der Bewegungsprogramme. Diese Entwicklung wird statistisch belegt und als signifikant interpretiert. Nach der Varianzanalyse mit zwei Messwiederholungen sind die Veränderungen in Bezug auf den Faktor Zeit hoch signifikant und sehr signifikant in Bezug auf die Interaktion Gruppe*Zeit. 
In der Follow-up-Periode zeichnet sich ein leichter Werterückgang für beide Gruppen ab. Dieses ist nach der varianzanalytischen Berechnung ein signifikantes Ergebnis bzgl. des Faktors Zeit.

Bei der dritten Kraftaufgabe „Jump and reach“ sind kaum Veränderungen zu finden. Es gibt weder wesentliche Gruppenunterschiede noch relevante Leistungsveränderungen bei beiden Gruppen während des gesamten Untersuchungszeitraumes (siehe Tabelle 42 und Abbildung 27).

Nach der zweifaktoriellen Varianzanalyse mit Messwiederholung ergibt sich eine Signifikanz bzgl. der Interaktion Gruppe *Zeit während der Treatmentperiode (siehe Tabelle 43). Während am Anfang des Treatments die Experimentalgruppe mit ihrem Wert leicht hinter der Kontrollgruppe lag, verbessert sie sich am Ende des Treatments und überholt die Kontrollgruppe, die einen minimalen Wertrückgang aufweist.

Tabelle 42 zeigt die Veränderung, der Mittelwerte der 3 Aufgaben des Krafttests über die gesamte Studienzeit:

Tabelle 42: Ergebnisse der 3 Krafttestaufgaben der beiden Gruppen zu den vier Messzeitpunkten, t0 = bei Aufnahme, $t 1=4$ Wochen nach Aufnahme (Kursbeginn), t2 $=8$ Wochen nach Kursbeginn (Kursende), $t 3=6$ Monate nach Kursende

\begin{tabular}{|c|c|c|c|c|}
\hline & t0 & t1 & t2 & T3 \\
\hline \multicolumn{5}{|l|}{ Experimentalgruppe $(n=30)$} \\
\hline $\begin{array}{c}\text { Anzahl Sit-ups } \\
\text { Arithmetisches Mittel } \\
\text { Standardabweichung (SD) }\end{array}$ & $\begin{array}{r}11,31 \\
\pm 5,63 \\
\end{array}$ & $\begin{array}{r}11,97 \\
\pm 5,57 \\
\end{array}$ & $\begin{array}{l}17,81 \\
\pm 4,02 \\
\end{array}$ & $\begin{array}{r}17,81 \\
\pm 3,99 \\
\end{array}$ \\
\hline $\begin{array}{l}\text { Anzahl der Liegestütze } \\
\text { Arithmetisches Mittel } \\
\text { Standardabweichung (SD) }\end{array}$ & $\begin{array}{l}14,52 \\
\pm 2,87\end{array}$ & $\begin{array}{l}15,87 \\
\pm 4,11\end{array}$ & $\begin{array}{l}22,41 \\
\pm 4,01\end{array}$ & $\begin{array}{l}21,68 \\
\pm 5,36 \\
\end{array}$ \\
\hline $\begin{array}{c}\text { Sprunghöhe in cm bei Jump and Reach } \\
\text { Arithmetisches Mittel } \\
\text { Standardabweichung (SD) }\end{array}$ & $\begin{array}{l}20,83 \\
\pm 5,09\end{array}$ & $\begin{array}{c}21, \\
\pm 6,01\end{array}$ & $\begin{array}{l}22,78 \\
\pm 6,82 \\
\end{array}$ & $\begin{array}{l}21,30 \\
\pm 5,13 \\
\end{array}$ \\
\hline \multicolumn{5}{|l|}{ Kontrollgruppe $(n=28)$} \\
\hline $\begin{array}{c}\text { Anzahl Sit-ups } \\
\text { Arithmetisches Mittel } \\
\text { Standardabweichung (SD) } \\
\end{array}$ & $\begin{array}{r}10,93 \\
\pm 6,32 \\
\end{array}$ & $\begin{array}{r}13,85 \\
\pm 6,86 \\
\end{array}$ & $\begin{array}{c}16,5 \\
\pm 6,24 \\
\end{array}$ & $\begin{array}{c}16,48 \\
\pm 7,9 \\
\end{array}$ \\
\hline $\begin{array}{l}\text { Anzahl der Liegestütze } \\
\text { Arithmetisches Mittel } \\
\text { Standardabweichung (SD) }\end{array}$ & $\begin{array}{l}16,19 \\
\pm 4,64\end{array}$ & $\begin{array}{l}17,56 \\
\pm 4,69\end{array}$ & $\begin{array}{l}22,38 \\
\pm 4,69\end{array}$ & $\begin{array}{l}20,11 \\
\pm 7,14\end{array}$ \\
\hline $\begin{array}{c}\text { Sprunghöhe in cm bei Jump and Reach } \\
\text { Arithmetisches Mittel } \\
\text { Standardabweichung (SD) }\end{array}$ & $\begin{array}{l}21,15 \\
\pm 5,82\end{array}$ & $\begin{array}{l}21,73 \\
\pm 6,49\end{array}$ & $\begin{array}{l}21,58 \\
\pm 6,55\end{array}$ & $\begin{array}{l}21,96 \\
\pm 7,91\end{array}$ \\
\hline
\end{tabular}


In der folgenden Abbildung ist der Verlauf der Ergebnisse der Kraftaufgabe „Situps“ graphisch dargestellt:

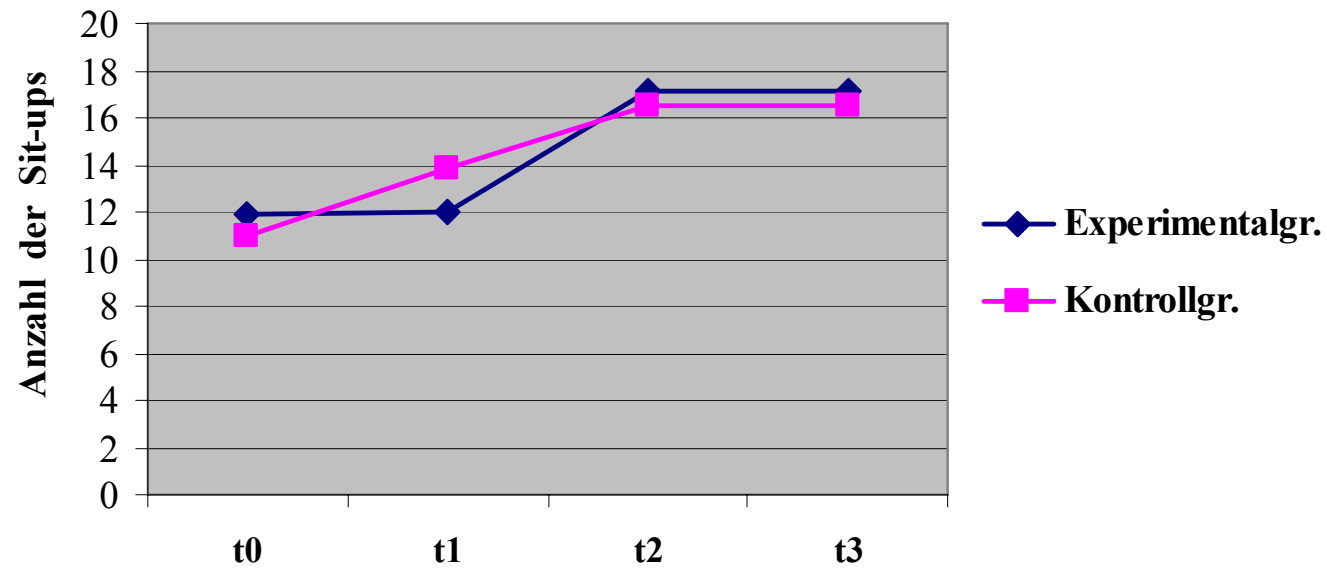

Abbildung 25: Verlauf der der Kraftaufgabe „Sit-ups“ der Experimentalgruppe $(\mathrm{n}=30)$ und Kontrollgruppe $(\mathrm{n}=28)$ über 4 Messzeitpunkte im Gruppenvergleich

In der folgenden Abbildung ist der Verlauf der Ergebnisse der Kraftaufgabe „Liegestütze“ graphisch dargestellt:

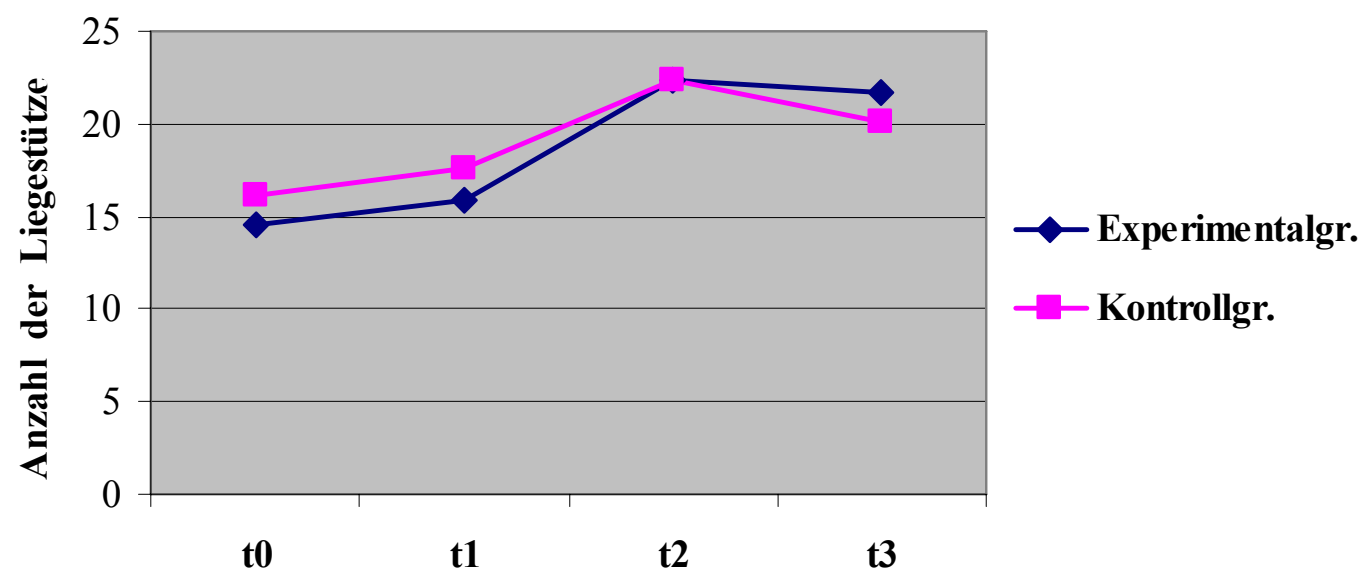

Abbildung 26: Verlauf der Kraftaufgabe „Liegestütze“ der Experimentalgruppe $(\mathrm{n}=30)$ und Kontrollgruppe $(\mathrm{n}=28)$ über 4 Messzeitpunkte im Gruppenvergleich

In der folgenden Abbildung ist der Verlauf der Ergebnisse der Kraftaufgabe „Jump and reach " graphisch dargestellt: 


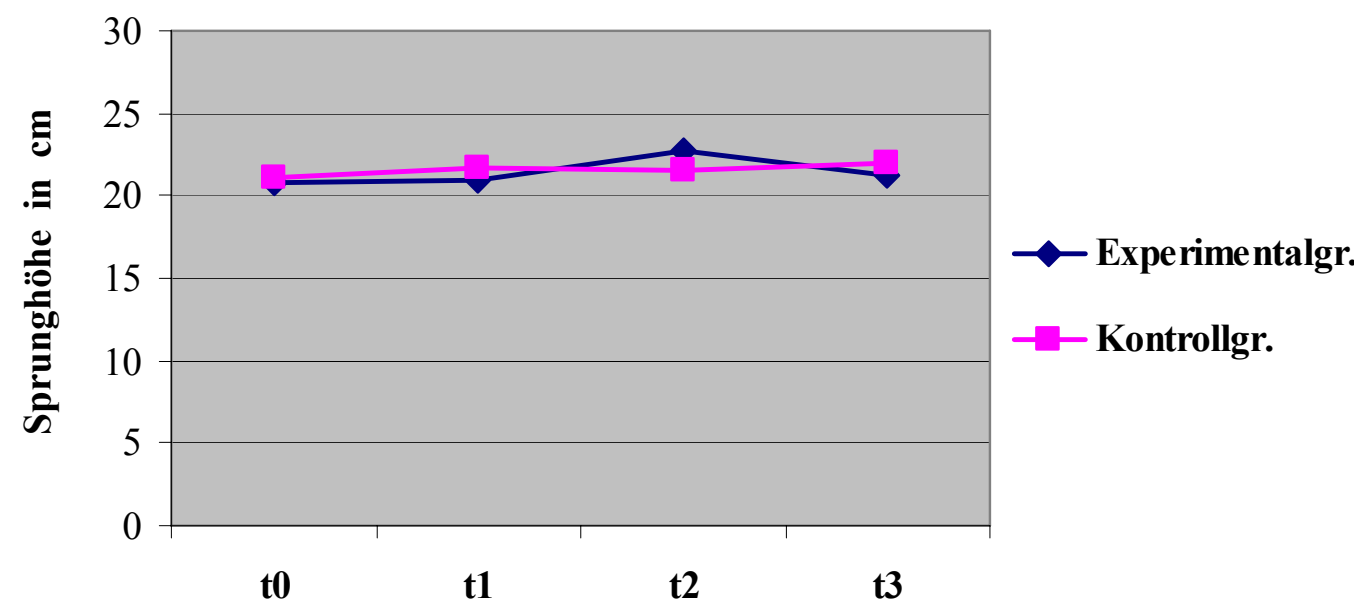

Abbildung 27: Verlauf der Kraftaufgabe „Jump and reach“ der Experimentalgruppe $(\mathrm{n}=30)$ und Kontrollgruppe $(\mathrm{n}=28)$ über 4 Messzeitpunkte im Gruppenvergleich

Die folgende Tabelle fasst die Ergebnisse der varianzanalytischen Testung zusammen:

Tabelle 43: Signifikante Ergebnisse der zweifaktoriellen Varianzanalyse mit Messwiederholungen der 3 Krafttestaufgaben über 4 Messzeitpunkte

\begin{tabular}{|l|l|l|l|}
\hline AV & Haupteffekt : Zeit & Signifikanz & Effektstärke $\left(\mathrm{Eta}^{2}\right)$ \\
\hline Sit-ups (t0-t1) & $\mathrm{F}=10,27$ & $\mathrm{p}=.002$ & $\eta^{2}=.160$ \\
\hline & Interaktion: Gruppe* Zeit & & \\
\hline Sit-ups (t0-t1) & $\mathrm{F}=4,13$ & $\mathrm{p}=.047$ & $\eta^{2}=.071$ \\
\hline & Haupteffekt : Zeit & & \\
\hline Sit-ups (t1-t2) & $\mathrm{F}=64,36$ & $\mathrm{p}=.000$ & $\eta^{2}=.558$ \\
\hline & Interaktion: Gruppe* Zeit & & \\
\hline Sit-ups (t1-t2) & $\mathrm{F}=10,09$ & $\mathrm{p}=.003$ & $\eta^{2}=.165$ \\
\hline
\end{tabular}

\begin{tabular}{|l|l|l|l|}
\hline AV & Haupteffekt : Zeit & Signifikanz & Effektstärke $\left(\mathrm{Eta}^{2}\right)$ \\
\hline Liegestütze (t0-t1) & $\mathrm{F}=11,07$ & $\mathrm{P}=.002$ & $\eta^{2}=.170$ \\
\hline & Haupteffekt : Zeit & & \\
\hline Liegestütze (t1-t2) & $\mathrm{F}=185,28$ & $\mathrm{p}=.000$ & $\eta^{2}=.784$ \\
\hline & Interaktion: Gruppe* Zeit & & \\
\hline Liegestütze (t1-t2) & $\mathrm{F}=0,15$ & $\mathrm{p}=.015$ & $\eta^{2}=.110$ \\
\hline & Haupteffekt : Zeit & & \\
\hline Liegestütze (t2-t3) & $\mathrm{F}=8,05$ & $\mathrm{P}=.007$ & $\eta^{2}=.141$ \\
\hline
\end{tabular}

\begin{tabular}{|l|l|l|l|}
\hline AV & Interaktion: Gruppe* Zeit & Signifikanz & Effektstärke $\left(\mathrm{Eta}^{2}\right)$ \\
\hline Jump and reach (t1-t2) & $\mathrm{F}=4,81$ & $\mathrm{p}=.033$ & $\eta^{2}=.088$ \\
\hline
\end{tabular}




\subsection{Das Bewegungsverhalten}

Die körperliche Aktivität wurde anhand einer durch den Verfasser vorgenommenen Modifikation des Fragebogens zur Erfassung der sportlichen Aktivität aus der Studie „Kommunale Gesundheitsförderung in Bad Schönborn“ erfasst (Woll, 2004).

Es werden drei Bereiche unterschieden:

- Sportliche Aktivität

- Körperliche Aktivität in der Freizeit (außer beim Sport)

- Körperliche Aktivität im Beruf

Bezüglich der aktuellen sportlichen Aktivität wurden die Selbstzuordnung zur Gruppe der „Sportler“ bzw. „Nicht-Sportler“ sowie die Art, Intensität und Häufigkeit erfasst.

Für die Berechnungen fehlen die Daten eines Teilnehmers der Experimentalgruppe zum Messzeitpunkt der Katamnese (t3).

Die folgende Tabelle stellt die Häufigkeit der sportlichen Aktivität der beiden Gruppen dar:

Tabelle 44: Relative und absolute Häufigkeit der sportlichen Aktivität der beiden Gruppen bei Aufnahme (t0) und 6 Monate nach Ende des Programms (t3)

\begin{tabular}{|c|c|c|}
\hline Experimentalgruppe $(n=30)$ & t0 & t3 \\
\hline $\begin{array}{c}\text { Sportler } \\
\text { Absolute Anzahl / Relative Häufigkeit (in \%) }\end{array}$ & $10(33,3 \%)$ & $22(75,9 \%)$ \\
\hline $\begin{array}{c}\text { Nicht - Sportler } \\
\text { Absolute Anzahl / Relative Häufigkeit (in \%) }\end{array}$ & $20(66,7 \%)$ & $7(24,1 \%)$ \\
\hline Kontrollgruppe $(n=28)$ & & \\
\hline $\begin{array}{c}\text { Sportler } \\
\text { Absolute Anzahl / Relative Häufigkeit (in \%) }\end{array}$ & $13(46,4 \%)$ & $16(57,1 \%)$ \\
\hline $\begin{array}{c}\text { Nicht - Sportler } \\
\text { Absolute Anzahl / Relative Häufigkeit (in \%) }\end{array}$ & $15(53,6 \%)$ & $12(42,9 \%)$ \\
\hline
\end{tabular}

In der folgenden Abbildung wird das Verhältnis von „Sportler“ und „Nicht-Sportler“ in den beiden Gruppen zu den 2 Messzeitpunkten graphisch dargestellt: 


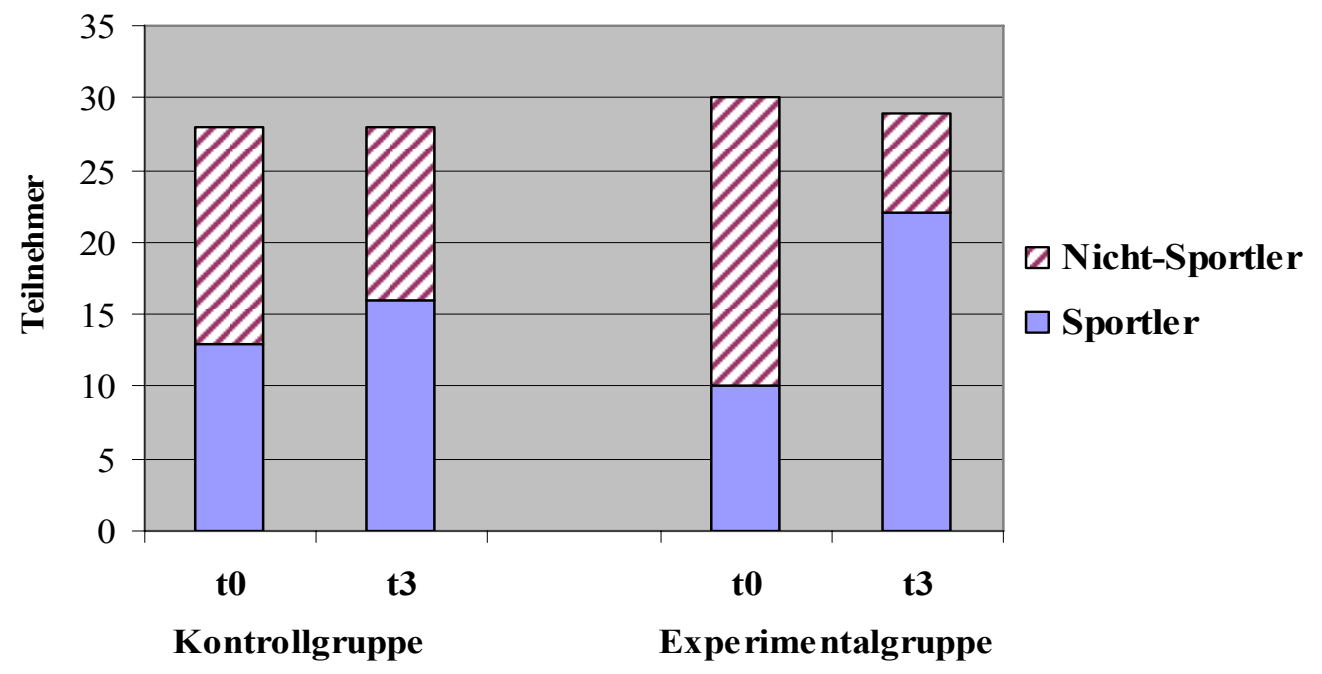

Abbildung 28: Verhältnis von „Sportler“ und „Nicht-Sportler“ in der Experimentalgruppe $(n=30)$ und Kontrollgruppe $(n=28)$ bei Aufnahme (t0) und 6 Monate nach Ende des Programms (t3) im Gruppenvergleich

Zur Selbsteinschätzung der Intensität wurde der subjektiv empfundene Anstrengungsgrad, die Anzahl der Trainingseinheiten pro Woche, die Dauer der Übungseinheiten sowie die Art der sportlichen Aktivität erfragt.

Tabelle 45: Relative und absolute Intensität der sportlichen Aktivität der „Sportler“ beider Gruppen bei Aufnahme (t0) und 6 Monate nach Ende des Programms (t3)

\begin{tabular}{|c|c|c|}
\hline Experimentalgruppe (n=30) & t0 & t3 \\
\hline $\begin{array}{c}\text { Locker und leicht } \\
\text { Absolute Anzahl / Relative Häufigkeit (in \%) }\end{array}$ & 0 & 0 \\
\hline $\begin{array}{c}\text { Flott und zügig } \\
\text { Absolute Anzahl / Relative Häufigkeit (in \%) }\end{array}$ & $8(80 \%)$ & $16(72,7 \%)$ \\
\hline $\begin{array}{c}\text { Hart und angestrengt } \\
\text { Absolute Anzahl / Relative Häufigkeit (in \%) }\end{array}$ & $2(20 \%)$ & $6(27,3 \%)$ \\
\hline Kontrollgruppe (n=28) & & $3(18,8 \%)$ \\
\hline $\begin{array}{c}\text { Locker und leicht } \\
\text { Absolute Anzahl / Relative Häufigkeit (in \%) }\end{array}$ & $5(38,5 \%)$ & $11(68,8 \%)$ \\
\hline $\begin{array}{c}\text { Flott und zügig } \\
\text { Absolute Anzahl / Relative Häufigkeit (in \%) }\end{array}$ & $8(61,5 \%)$ & $2(12,5 \%)$ \\
\hline $\begin{array}{c}\text { Hart und angestrengt } \\
\text { Absolute Anzahl / Relative Häufigkeit (in \%) }\end{array}$ & 0 & \\
\hline
\end{tabular}

Die folgende Tabelle zeigt die Häufigkeit der sportlichen Aktivität (pro Woche) bei „Sportlern“ der beiden Gruppen: 
Tabelle 46: Relative und absolute Häufigkeit der sportlichen Aktivität der „Sportler“ beider Gruppen bei Aufnahme (t0) und 6 Monate nach Ende des Programms (t3)

\begin{tabular}{|c|c|c|}
\hline Experimentalgruppe (n=30) & t0 & t3 \\
\hline $\begin{array}{c}<1 \mathrm{x} / \text { Woche } \\
\text { Absolute Anzahl / Relative Häufigkeit (in \%) }\end{array}$ & 0 & 0 \\
\hline $\begin{array}{c}\text { 1X/Woche } \\
\text { Absolute Anzahl / Relative Häufigkeit (in \%) }\end{array}$ & $6(60 \%)$ & $9(40,9 \%)$ \\
\hline $\begin{array}{c}\text { 2x/Woche } \\
\text { Absolute Anzahl / Relative Häufigkeit (in \%) }\end{array}$ & $2(20 \%)$ & $6(27,3 \%)$ \\
\hline $\begin{array}{c}3 \times \text { Woche u. m. } \\
\text { Absolute Anzahl / Relative Häufigkeit (in \%) }\end{array}$ & $2(20 \%)$ & $7(31,8 \%)$ \\
\hline Kontrollgruppe (n=28) & & $1(6,3 \%)$ \\
\hline $\begin{array}{c}<1 \times / \text { Woche } \\
\text { Absolute Anzahl / Relative Häufigkeit (in \%) }\end{array}$ & $1(7,7 \%)$ & $10(62,5 \%)$ \\
\hline $\begin{array}{c}\text { 1x/Woche } \\
\text { Absolute Anzahl / Relative Häufigkeit (in \%) }\end{array}$ & $9(69,2 \%)$ & $4(25 \%)$ \\
\hline $\begin{array}{c}\text { 2x/Woche } \\
\text { Absolute Anzahl / Relative Häufigkeit (in \%) }\end{array}$ & $1(7,7 \%)$ & $1(6,3 \%)$ \\
\hline $\begin{array}{c}3 x / \text { Woche u. m. } \\
\text { Absolute Anzahl / Relative Häufigkeit (in \%) }\end{array}$ & $2(15,4 \%)$ & \\
\hline
\end{tabular}

Die folgende Tabelle zeigt die Häufigkeit der Dauer der Übungseinheiten (Minuten pro Übungseinheit) bei Sportlern der beiden Gruppen:

Tabelle 47: Relative und absolute Häufigkeit der Dauer der Übungseinheiten der „Sportler" beider Gruppen bei Aufnahme (t0) und 6 Monate nach Ende des Programms (t3)

\begin{tabular}{|c|c|c|}
\hline Experimentalgruppe (n=30) & t0 & t3 \\
\hline $\begin{array}{c}\text { unter 20 Min } \\
\text { Absolute Anzahl / Relative Häufigkeit (in \%) }\end{array}$ & 0 & 0 \\
\hline $\begin{array}{c}\text { 20 - 40 Min } \\
\text { Absolute Anzahl / Relative Häufigkeit (in \%) }\end{array}$ & $2(20 \%)$ & $4(18,2 \%)$ \\
\hline $\begin{array}{c}\text { mehr als 40 Min } \\
\text { Absolute Anzahl / Relative Häufigkeit (in \%) }\end{array}$ & $8(80 \%)$ & $18(81,8 \%)$ \\
\hline Kontrollgruppe (n=28) & 0 & $1(6,3 \%)$ \\
\hline $\begin{array}{c}\text { unter 20 Min } \\
\text { Absolute Anzahl / Relative Häufigkeit (in \%) }\end{array}$ & $7(53,8 \%)$ & $8(50 \%)$ \\
\hline $\begin{array}{c}20-40 \text { Min } \\
\text { Absolute Anzahl / Relative Häufigkeit (in \%) }\end{array}$ & $6(46,2 \%)$ & $7(43,8 \%)$ \\
\hline $\begin{array}{c}\text { mehr als 40 Min } \\
\text { Absolute Anzahl / Relative Häufigkeit (in \%) }\end{array}$ & & \\
\hline
\end{tabular}


Die folgende Tabelle dokumentiert die am häufigsten betriebenen Sportarten bei den sportlich Aktiven. Bei der Befragung wurden insgesamt 12 Sportarten genannt. Mehrfachnennungen waren bei der Befragung möglich.

Tabelle 48: Relative und absolute Häufigkeit der Sportarten beider Gruppen bei Aufnahme (t0) und 6 Monate nach Ende des Programms (t3); $(N=58)$

\begin{tabular}{|c|c|c|}
\hline Sportarten & Anzahl zu to & Anzahl zu t3 \\
\hline Gymnastik & $5(17,2 \%)$ & $13(23,6 \%)$ \\
\hline Schwimmen & $10(34,5 \%)$ & $10(18,2 \%)$ \\
\hline Radfahren & $3(10,3 \%)$ & $6(10,9 \%)$ \\
\hline Walking & $1(3,4 \%)$ & $11(20,0 \%)$ \\
\hline Krafttraining & $1(3,4 \%)$ & $5(9,1 \%)$ \\
\hline Bergwandern & $2(6,9 \%)$ & $3(5,5 \%)$ \\
\hline Aerobics & $1(3,4 \%)$ & $1(1,8 \%)$ \\
\hline Jogakurs & $1(3,4 \%)$ & $2(3,6 \%)$ \\
\hline Tanzen & $3(10,3 \%)$ & $2(3,6 \%)$ \\
\hline Tennis - Badminton & $1(3,4 \%)$ & $1(1,8 \%)$ \\
\hline Feldenkreis & $1(3,4 \%)$ & 0 \\
\hline Taibo & 0 & $1(1,8 \%)$ \\
\hline
\end{tabular}

Die Befragung zur aktuellen sportlichen Aktivität erlaubt neben der einfachen Verneinung bzw. Bejahung eine differenzierte Betrachtung der Ergebnisse.

Der Fragebogen ermöglicht es, einen Index bzgl. der aktuellen sportlichen Aktivität zu bilden.

Der Index zur aktuellen sportlichen Aktivität wird über Fragen nach der Häufigkeit der wöchentlichen Trainings- und Bewegungseinheiten, deren Dauer und der subjektiv empfundenen Intensität der Belastung im Training ermittelt (Bässler, 1988, S. 194, zitiert nach Woll, 2004, S. 7). 
Anhand dieser Angaben zur aktuellen sportlichen Aktivität werden folgende Typen gebildet:

- Nichtsportler:

- Gelegenheitssportler:

- Regelmäßige Sportler:

- Intensivsportler: treiben keinen Sport

weniger als eine Stunde/Woche sportlich aktiv

mindestens 1 Stunde/Woche sportlich aktiv

mindestens dreimal 40 Minuten/Woche mit

mindestens mittlere Intensität sportlich aktiv

Tabelle 49: Relative und absolute Häufigkeit der Sportlertypen in beiden Gruppen bei Aufnahme (t0) und 6 Monate nach Ende des Programms (t3)

\begin{tabular}{|c|c|c|}
\hline Experimentalgruppe (n=30) & t0 & t3 \\
\hline $\begin{array}{c}\text { Nichtsportler } \\
\text { Absolute Anzahl / Relative Häufigkeit (in \%) }\end{array}$ & $20(66,7 \%)$ & $7(24,1 \%)$ \\
\hline $\begin{array}{c}\text { Gelegenheitssportler } \\
\text { Absolute Anzahl / Relative Häufigkeit (in \%) }\end{array}$ & $2(6,7 \%)$ & $3(10,3 \%)$ \\
\hline $\begin{array}{c}\text { Regelmäßige Freizeitsportler } \\
\text { Absolute Anzahl / Relative Häufigkeit (in \%) }\end{array}$ & $6(20,0 \%)$ & $12(41,4 \%)$ \\
\hline $\begin{array}{c}\text { Intensivsportler } \\
\text { Absolute Anzahl / Relative Häufigkeit (in \%) }\end{array}$ & $2(6,7 \%)$ & $7(24,1 \%)$ \\
\hline Kontrollgruppe (n=28) & $15(53,6 \%)$ & $12(42,9 \%)$ \\
\hline $\begin{array}{c}\text { Nichtsportler } \\
\text { Absolute Anzahl / Relative Häufigkeit (in \%) }\end{array}$ & $5(17,9 \%)$ & $7(25,0 \%)$ \\
\hline $\begin{array}{c}\text { Gelegenheitssportler } \\
\text { Absolute Anzahl / Relative Häufigkeit (in \%) }\end{array}$ & $8(28,6 \%)$ & $8(28,6 \%)$ \\
\hline $\begin{array}{c}\text { Regelmäßige Freizeitsportler } \\
\text { Absolute Anzahl / Relative Häufigkeit (in \%) }\end{array}$ & $1(3,6 \%)$ \\
\hline $\begin{array}{c}\text { Intensivsportler } \\
\text { Absolute Anzahl / Relative Häufigkeit (in \%) }\end{array}$ & 0 & \\
\hline
\end{tabular}

Darüber hinaus wird in der vorliegenden Studie zwischen aktueller und habitueller sportlicher Aktivität unterschieden. Die habituelle sportliche Aktivität, d.h. die Kontinuität des Sporttreibens über die Lebenspanne hinweg, wird durch retrospektive Angaben zur sportlichen Aktivität im Vergleich zu aktuellen sportlichen Aktivitäts-Typen gebildet.

Die habituelle sportliche Aktivität wurde über folgende Fragen operationalisiert:

- Haben Sie seit Ihrer Schulzeit durchgehend Sport getrieben?

- Haben Sie vor 10 Jahren keinen, weniger, gleichviel oder mehr Sport getrieben als heute?

- Blicken Sie in die Zukunft. Glauben Sie, dass Sie in 10 Jahren Sport treiben werden? 
Tabelle 50: Relative und absolute Häufigkeit der habituellen sportlichen Aktivität beider Gruppen bei Aufnahme (t0)

\begin{tabular}{|c|c|c|}
\hline & \multicolumn{2}{|c|}{ t0 } \\
\hline & $\begin{array}{c}\text { Experimentalgruppe } \\
(\mathbf{n = 3 0 )}\end{array}$ & $\begin{array}{c}\text { Kontrollgruppe } \\
(\mathbf{n = 2 8})\end{array}$ \\
\hline $\begin{array}{c}\text { Seit Schule Sport } \\
\text { Absolute Anzahl / Relative Häufigkeit (in \%) }\end{array}$ & $3(11,1 \%)$ & $1(3,6 \%)$ \\
\hline $\begin{array}{c}\text { Vor 10 Jahren Sport } \\
\text { Absolute Anzahl / Relative Häufigkeit (in \%) }\end{array}$ & $14(46,7 \%)$ & $12(42,9 \%)$ \\
\hline $\begin{array}{c}\text { In 10 Jahren Sport } \\
\text { Absolute Anzahl / Relative Häufigkeit (in \%) }\end{array}$ & $30(100 \%)$ & $28(100 \%)$ \\
\hline
\end{tabular}

Bei den Teilnehmern, die vor zehn Jahren Sport getrieben haben, wurde das Ausmaß der damaligen sportlichen Aktivität im Vergleich mit der aktuellen Aktivität erfragt.

Tabelle 51: Relative und absolute Häufigkeit des Ausmaßes der sportlichen Aktivität vor zehn Jahren (Teilnehmer, die vor 10 Jahren sportlich aktiv waren) der beiden Gruppen bei Aufnahme (to)

\begin{tabular}{|c|c|c|}
\hline & \multicolumn{2}{|c|}{ t0 } \\
\hline & $\begin{array}{c}\text { Experimentalgruppe } \\
(\mathbf{n = 3 0 )}\end{array}$ & $\begin{array}{c}\text { Kontrollgruppe } \\
(\mathbf{n = 2 8})\end{array}$ \\
\hline $\begin{array}{c}\text { Weniger } \\
\text { Absolute Anzahl / Relative Häufigkeit (in \%) }\end{array}$ & $2(14,3 \%)$ & $2(16,7 \%)$ \\
\hline $\begin{array}{c}\text { Genauso viel } \\
\text { Absolute Anzahl / Relative Häufigkeit (in \%) }\end{array}$ & 0 & $2(16,7 \%)$ \\
\hline $\begin{array}{c}\text { Mehr } \\
\text { Absolute Anzahl / Relative Häufigkeit (in \%) }\end{array}$ & $12(85,7 \%)$ & $8(66,7 \%)$ \\
\hline
\end{tabular}

Durch diese Angaben konnte ein Index der habituellen sportlichen Aktivität erstellt werden. Aus dem Vergleich der vergangenen mit der gegenwärtigen sportlichen Aktivität der Teilnehmer wurden folgende Gruppen gebildet:

- Nichtsportler: Treiben heute und trieben früher keinen Sport.

- Abbrecher: Treiben heute keinen Sport, haben aber vor 10 Jahren Sport getrieben.

- Einsteiger: Treiben heute Sport, haben jedoch vor 10 Jahren keinen Sport getrieben.

- Regelmäßig Sporttreibende: Personen, die heute Sport treiben und vor 10 Jahren Sport getrieben haben. 
Tabelle 52: Relative und absolute Häufigkeit der habituellen Sportlertypen beider Gruppen bei Aufnahme (t0)

\begin{tabular}{|c|c|c|}
\hline & \multicolumn{2}{|c|}{ t0 } \\
\hline & $\begin{array}{c}\text { Experimentalgruppe } \\
\text { (n=30) }\end{array}$ & $\begin{array}{c}\text { Kontrollgruppe } \\
\text { (n=28) }\end{array}$ \\
\hline $\begin{array}{c}\text { Nichtsportler } \\
\text { Absolute Anzahl / Relative Häufigkeit (in \%) }\end{array}$ & $13(43,3 \%)$ & $12(42,9 \%)$ \\
\hline $\begin{array}{c}\text { Abbrecher } \\
\text { Absolute Anzahl / Relative Häufigkeit (in \%) }\end{array}$ & $7(23,3 \%)$ & $3(10,7 \%)$ \\
\hline $\begin{array}{c}\text { Einsteiger } \\
\text { Absolute Anzahl / Relative Häufigkeit (in \%) }\end{array}$ & $3(10,0 \%)$ & $4(14,3 \%)$ \\
\hline Regelmäßig Sporttreibende \\
Absolute Anzahl / Relative Häufigkeit (in \%)
\end{tabular}

Die außersportlichen körperlichen Freizeitaktivitäten werden durch die Befragung nach den täglichen Geh- und Radstrecken und nach der Ausübung von körperlich anstrengenden Freizeittätigkeiten erfasst.

Tabelle 53: Relative und absolute Häufigkeit der täglich zurückgelegten Fußstrecke (werktags) der beiden Gruppen bei Aufnahme (t0) und 6 Monate nach Ende des Programms (t3)

\begin{tabular}{|c|c|c|}
\hline Experimentalgruppe $(n=30)$ & t0 & t3 \\
\hline $\begin{array}{c}\text { Fast keine } \\
\text { Absolute Anzahl / Relative Häufigkeit (in \%) }\end{array}$ & $1(3,3 \%)$ & $1(3,4 \%)$ \\
\hline $\begin{array}{c}\text { Weniger als } 1 \mathrm{~km} \\
\text { Absolute Anzahl / Relative Häufigkeit (in \%) }\end{array}$ & $2(6,7 \%)$ & $1(3,4 \%)$ \\
\hline $\begin{array}{c}1 \text { - } 2 \mathrm{~km} \\
\text { Absolute Anzahl / Relative Häufigkeit (in \%) }\end{array}$ & $16(53,3 \%)$ & $10(34,5 \%)$ \\
\hline $\begin{array}{c}3 \text { - } 5 \mathrm{~km} \\
\text { Absolute Anzahl / Relative Häufigkeit (in \%) }\end{array}$ & $10(33,3 \%)$ & $16(55,2 \%)$ \\
\hline $\begin{array}{c}6-9 \mathrm{~km} \\
\text { Absolute Anzahl / Relative Häufigkeit (in \%) }\end{array}$ & $1(3,3 \%)$ & $1(3,4 \%)$ \\
\hline $\begin{array}{l}\qquad 10 \mathrm{~km} \text { und mehr } \\
\text { Absolute Anzahl / Relative Häufigkeit (in \%) }\end{array}$ & 0 & 0 \\
\hline \multicolumn{3}{|l|}{ Kontrollgruppe $(n=28)$} \\
\hline $\begin{array}{c}\text { Fast keine } \\
\text { Absolute Anzahl / Relative Häufigkeit (in \%) }\end{array}$ & $1(3,6 \%)$ & 0 \\
\hline $\begin{array}{c}\text { Weniger als } 1 \mathrm{~km} \\
\text { Absolute Anzahl / Relative Häufigkeit (in \%) }\end{array}$ & $2(7,1 \%)$ & $2(7,1 \%)$ \\
\hline $\begin{array}{c}1 \text { - } 2 \mathrm{~km} \\
\text { Absolute Anzahl / Relative Häufigkeit (in \%) }\end{array}$ & $16(57,1 \%)$ & $15(53,6 \%)$ \\
\hline $\begin{array}{c}3-5 \mathrm{~km} \\
\text { Absolute Anzahl / Relative Häufigkeit (in \%) }\end{array}$ & $9(32,1 \%)$ & $8(28,6 \%)$ \\
\hline $\begin{array}{c}6-9 \mathrm{~km} \\
\text { Absolute Anzahl / Relative Häufigkeit (in \%) }\end{array}$ & 0 & $3(10,7 \%)$ \\
\hline $\begin{array}{l}10 \mathrm{~km} \text { und mehr } \\
\text { Absolute Anzahl / Relative Häufigkeit (in \%) }\end{array}$ & 0 & 0 \\
\hline
\end{tabular}


Tabelle 54: Relative und absolute Häufigkeit Außersportliche Benutzung des Fahrrades (werktags) der beiden Gruppen bei Aufnahme (t0), und 6 Monate nach Ende des Programms (t3)

\begin{tabular}{|c|c|c|}
\hline Experimentalgruppe (n=30) & t0 & t3 \\
\hline $\begin{array}{c}\text { Fahrradbenutzer } \\
\text { Absolute Anzahl / Relative Häufigkeit (in \%) }\end{array}$ & $10(33,3 \%)$ & $13(44,8 \%)$ \\
\hline Kontrollgruppe (n=28) & & \\
\cline { 1 - 1 } $\begin{array}{c}\text { Fahrradbenutzer } \\
\text { Absolute Anzahl / Relative Häufigkeit (in \%) }\end{array}$ & $10(35,7 \%)$ & $12(44,4 \%)$ \\
\hline
\end{tabular}

Tabelle 55: Relative und absolute Häufigkeit der Teilnehmer beider Gruppen die körperlich anstrengende Freizeittätigkeit ausführen bei Aufnahme (t0) und 6 Monate nach Ende des Programms ( $t 3)$

\begin{tabular}{|c|c|c|}
\hline Experimentalgruppe (n=30) & t0 & $\mathbf{t 3}$ \\
\hline $\begin{array}{c}\text { Anstrengende Freizeittätigkeit } \\
\text { Absolute Anzahl / Relative Häufigkeit (in \%) }\end{array}$ & $8(26,7)$ & $10(34,5 \%)$ \\
\hline Kontrollgruppe (n=28) & & \\
\hline $\begin{array}{c}\text { Anstrengende Freizeittätigkeit } \\
\text { Absolute Anzahl / Relative Häufigkeit (in \%) }\end{array}$ & $8(28,6 \%)$ & $12(42,9 \%)$ \\
\hline
\end{tabular}





\section{Diskussion}

In der vorliegenden Studie wurden die Effekte eines ambulanten Behandlungsprogramms für Menschen mit Adipositas unter sport- und bewegungswissenschaftlichen Fragestellungen untersucht.

Das Hauptaugenmerk dieser Arbeit galt der Frage, ob durch das spezifische psychoedukative bewegungstherapeutische Programm der Verlauf der Adipositas günstiger beeinflusst werden kann als durch ein reines Fitnesstraining.

Die Effekte wurden mit der Eigenwartekontrollgruppe sowie mit einer Kontrollgruppe verglichen. Im Folgenden werden die Ergebnisse interpretiert und mit dem im theoretischen Teil dargestellten aktuellen Forschungsstand in Verbindung gebracht. Methodenkritische Aspekte werden unter dem entsprechenden Untersuchungsparameter für jedes Verfahren diskutiert.

\subsection{Körpergewicht, Body Mass Index und Körperzusammensetzung}

Das Hauptziel in der Adipositasbehandlung liegt in einer langfristigen Gewichtsstabilisierung auf einem niedrigeren Gewichtsniveau. Das Erreichen des Normalgewichtes stellt in den meisten Fällen kein realistisches Therapieziel dar. Eine mäßige Gewichtssenkung um 5 bis $10 \%$ des Ausgangsgewichts, über mindestens ein Jahr gehalten, gilt als Therapieerfolg (DAG, 2006).

Die Gewichtsreduktion in der vorliegenden Studie beträgt $440 \mathrm{~g}$ für die Experimentalgruppe und $550 \mathrm{~g}$ für die Kontrollgruppe in der Baseline. Diese Gewichtsreduktion der beiden Gruppen während der Baselineperiode ist statistisch signifikant (Zeiteffekt). Sie lässt sich in einer Wartekontrollzeit dadurch erklären, dass schon die Entscheidung für eine Intervention positive Effekte bewirkt. Allerdings ist die Effektstärke dermaßen gering, dass auf eine Interpretation dieser Signifikanz verzichtet wird. Eine Steigerung der körperlichen Aktivitäten in dieser Zeitspanne wurde nicht belegt.

Über den Zeitraum der Behandlung verloren die Teilnehmer an der Studie weitere 2,01 kg (EG) bzw. 1,02 kg (KG). Dieses ist eine signifikante Veränderung, die nach der für die Behandlungszeit berechneten Effektstärke von $\eta^{2}=0,366$ statistisch als ein großer Effekt zu beurteilen ist. Dennoch liegen diese Werte damit deutlich unter einer als erfolgreich zu bewertenden Gewichtsreduktion. 
$\mathrm{Zu}$ erklären ist dies dadurch, dass es sich hier im Gegensatz zu den empfohlenen multimodalen Therapieansätzen um die Effekte eine Monotherapie handelt. Es wurden während der gesamten Untersuchungszeit keine diätetischen Maßnahmen durchgeführt. Reduktionskostversuche in Eigenregie außerhalb des Programms wurden erfasst, sie spielten aber keine wesentlich Rolle und verteilten sich gleichermaßen auf die Versuchs- als auch auf die Kontrollgruppe. Die geringen Effekte auf die Gewichtsreduktion sind mit den Ergebnissen anderer, rein sporttherapeutisch ausgerichteter Interventionen vergleichbar.

Nach einer Metaanalyse von Miller et al. (1997) beträgt die Gewichtsabnahme bei Studien mit sporttherapeutischen Interventionen bei einer Behandlungsdauer von etwa 20 Wochen etwa 2,9 kg. Die untersuchte Gesamtstichprobe der sporttherapeutischen Studien hatte jedoch einen niedrigeren (BMI 26 - 35 Mittelwert 26,4), und einen niedrigen Körperfettanteil (28-38 \%) (Miller et al., 1997).

Die in dieser Arbeit beschriebene Gewichtsreduktion ist zwar prozentual mit der der Metaanlyse von Miller vergleichbar, in Relation zu den Ausgangsgewichten von 108 kg bzw. $112 \mathrm{~kg}$ hätte sie aber in den absoluten Werten höher ausfallen können.

Die Gewichtsreduktion während der Behandlungszeit ist mit 2,01 kg für die EG und $1,02 \mathrm{~kg}$ für die KG unterschiedlich, jedoch im Gruppenvergleich nicht signifikant. Nach Ende der Behandlung verzeichneten beide Gruppen wieder eine Gewichtszunahme. Es wird deutlich, dass auch bei dieser geringeren Gewichtsreduktion das Problem der Aufrechterhaltung des verlorenen Gewichtes nach der Beendung einer therapeutischen Maßnahme besteht (vgl. Kap. 4.5).

Diese Gewichtszunahme während der Follow-up-Periode ist bei der Experimentalgruppe wesentlich geringer als bei der Kontrollgruppe, die nach dem letzten Messzeitpunkt fast das Ausgangsgewicht erreicht. Der BMI zeigt hier einen statistisch signifikanten Gruppenunterschied. Der Gruppenunterschied in dieser Phase kann mit der Erhaltung der körperlichen Aktivitäten erklärt werden. Die Teilnehmer der Experimentalgruppe geben an, sportlich aktiver $\mathrm{zu}$ sein als die Teilnehmer der Kontrollgruppe. Dieses unterstützt die allgemeine These, dass ein wesentlicher Nutzen des körperlichen Trainings darin besteht, das Gewicht nach einer Reduktion zu erhalten.

Zusätzlich zu den Messungen des Körpergewichts wurden mit Hilfe der bioelektrischen Impedanzanalyse die Effekte des Bewegungsprogramms auf die 
verschiedenen Komponenten der Körperzusammensetzung untersucht (vgl. Kap. 5.3.2).

Zunächst konnte die fettfreie Körpermasse gemessen werden. Es war deutlich zu sehen, dass die fettfreie Körpermasse während der gesamten Studienzeit stabil blieb. Die Veränderungen bewegten sich im Rahmen von 20-440 g. Sie waren statistisch nicht signifikant und sind in Relation zur gesamten fettfreien Körpermasse von durchschnittlich ca. $61 \mathrm{~kg}$ in beiden Gruppen geringfügig und damit $\mathrm{zu}$ vernachlässigen. Diese Befunde stimmen mit der These überein, dass körperliches Training während eines Gewichtsreduktionsprogramms dem Abbau der Muskelmasse vorbeugt. In der vorliegenden Studie blieb der Verlust an fettfreier Körpermasse sehr gering, es gab teilweise minimale Zunahmen. Dieses Ergebnis deckt sich mit anderen Untersuchungen, die ohne Kalorienrestriktion arbeiten (Vögele, 2003; Votruba et al., 2000).

Der Gewichtsverlust während der Baselineperiode erklärt sich durch die Abnahme der Fettmasse. Diese Verluste mit -0,880 kg für die Experimentalgruppe und -0,460 $\mathrm{kg}$ für die Kontrollgruppe sind signifikant bei einer mittleren Effektstärke. In Relation zu dem gesamten Körperfettanteil ( $\mathrm{EG}=47 \mathrm{~kg}$ und $\mathrm{KG}=51 \mathrm{~kg}$ ) ist der Verlust des Körperfetts gering und kann zwar durch die mit einer Intervention verbundenen Erwartungen erklärt werden, sollte aber nicht überbewertet werden.

Während der Behandlungszeit wurden stärkere Verluste an Fettmasse für beide Gruppen ermittelt; sie machen fast den gesamten Gewichtsverlust aus. Diese Befunde stimmen mit der These überein, dass regelmäßige körperliche Aktivität während der Gewichtsreduktionsphase vorwiegend $\mathrm{zu}$ einer Reduktion von Körperfett führt (vgl. Miller et al. 1997; Vögele, 2003).

Der Fettverlust der Experimentalgruppe beträgt mit 2,11 kg von insgesamt 2,01 kg (105\%), während bei der Kontrollgruppe mit 0,96 kg von insgesamt 1,02 kg (94 \%) gemessen wurden. Die Experimentalgruppe (EG) verliert fast das Doppelte an Körperfettanteil gegenüber der Kontrollgruppe (KG). Hier handelt es sich nach der ANOVA um einen Interaktionseffekt, der bei gleichen Ausgangswerten der Gruppen auf eine unterschiedliche Entwicklung der beiden Gruppen hinweist. Bei einem $\eta^{2}=0,074$ ist dies als mittelstarker Effekt zu sehen.

Das psychoedukative Bewegungsprogramm scheint bzgl. der Gewichtsabnahme und der Fettreduktion über die Behandlungszeit effektiver $\mathrm{zu}$ sein als das reine Fitnessprogramm. 
Während des Katamnesezeitraums nehmen beide Gruppen wieder an Körperfett zu. Bei der Kontrollgruppe ist die Körperfettzunahme mit -0,970 kg sogar höher als die Gesamtgewichtszunahme dieses Zeitraums (-0,593 kg). Ähnlich verhält es sich auch in der Kontrollgruppe mit einer Fettreduktion von 1,52 kg bei einer Gesamtgewichtszunahme im Katamnesezeitraum von 1,2 kg. Die Gewichtszunahme in der Followup-Periode ist somit auf eine Zunahme des Körperfettanteils und auf einen Abbau der Muskelmasse zurückzuführen. Diese Veränderung der Körperzusammensetzung zugunsten des Körperfettes ist statistisch signifikant.

Der berechnete mittelstarke $\left(\eta^{2}=0,071\right)$ Gruppenunterschied zeigt, dass die Fettzunahme in der Experimentalgruppe signifikant geringer als in der Kontrollgruppe ist. Die Beobachtung des Bewegungsverhaltens führt dazu, diesen Gruppenunterschied als Folge einer vermehrten körperlichen Aktivität der Teilnehmer der Experimentalgruppe zu sehen. Ein Ziel des psychoedukativen Programms, die vermehrte Weiterführung des körperlichen Trainings über die Wissensvermittlung, könnte somit belegt sein.

Zusammenfassend ist festzustellen, dass beide Bewegungsprogramme einen mäßigen Einfluss hinsichtlich einer Gewichtsreduktion hatten. Beide Bewegungsprogramme führten zu Veränderungen der Körperzusammensetzung. Die fettfreie Körpermasse wurde trotz der Gewichtsreduktion erhalten, Körperfettmasse wurde abgebaut. Das psychoedukative Bewegungsprogramm hat eine größere Wirkung hinsichtlich der Fettreduktion. Auch die geringere Gewichtszunahme in der Katamnese (6 Monate nach der Behandlung) weist auf eine therapeutische Überlegenheit der Experimentalgruppe (psychoedukatives Bewegungsprogramm) gegenüber der Kontrollgruppe (Fitnesstraining) hin.

\subsection{Lebensqualität}

Die Datenlage ist eindeutig. Adipositas hat nicht nur einen negativen Einfluss auf die Gesundheit, sondern auch eine verheerende Wirkung auf die subjektive gesundheitsbezogene Lebensqualität (Fontaine \& Bartlett, 2003).

Zur Erfassung der Lebensqualität wurde wie beschrieben mit dem SF-36 ein allgemein anerkannter und weit verbreiteter standardisierter Fragebogen angewandt (Wadden \& Phelan 2002; Seidell, \& Tijhuis, 2002). Durch seine breite Anwendung 
liegen umfangreiche Vergleichsdaten verschiedener Patientenpopulationen wie auch der deutsche Normpopulation vor.

Während der Baseline gab es bzgl. der Lebensqualität mit Ausnahme der Skala „Vitalität“ keine signifikanten Veränderungen. Es ist auffällig, dass für diese Subskala von beiden Gruppen sehr niedrige Werte angegeben wurden. Es handelt sich um einen signifikanten Gruppenunterschied, der die Vergleichbarkeit der Gruppen hinsichtlich dieser Subskala erschwert. Die Kontrollgruppe weist in der „Vitalität“ niedrigere Werte als die Experimentalgruppe aus.

Während der Behandlungsperiode verbesserten sich sieben der acht Skalen des SF36 in der Gesamtstichprobe signifikant in mittlerem und starkem Ausmaß. Zusätzlich fanden sich signifikante Gruppenunterschiede in den Skalen „Vitalität“ und „Psychisches Wohlbefinden“. Bei der Skala „Körperliche Rollenfunktion“ zeigte sich ein signifikanter Interaktionseffekt.

Das Ansteigen der meisten Subskalen in beiden Gruppen weist auf den günstigen Einfluss beider Behandlungsverfahren hin. Sie führen beide $\mathrm{zu}$ einem generell verbesserten Gesundheitszustand.

Die Werte der Subskala „Vitalität“ steigen für beide Gruppen, liegen jedoch bei der Kontrollgruppe niedriger als bei der Experimentalgruppe. Ähnlich verhält es sich mit der Skala „Psychisches Wohlbefinden“. Trotz eines Anstiegs der Werte in beiden Gruppen liegt die Experimentalgruppe mit ihren Werten signifikant höher als die Kontrollgruppe.

Im Bezug auf die Skala „Körperliche Rollenfunktion“ verbessern sich zunächst beide Gruppen (signifikanter Zeiteffekt). Diese Verbesserung macht sich insbesondere bei der Kontrollgruppe bemerkbar, die einen deutlich schlechteren Ausgangswert als die Kontrollgruppe hatte.

Durch das Fitnessprogramm wird dieser Unterschied am Ende des Treatments ausgeglichen. Bezüglich der „Körperlichen Rollenfunktion“ wurde durch das reine Fitnesstraining eine deutlichere Veränderung erreicht als durch die psychoedukative Behandlungskomponente. Ob dieses an einer höheren Wirksamkeit des Fitnesstrainings oder an den unterschiedlichen Ausgangswerten in den beiden Gruppen liegt, kann nicht gesagt werden.

Am Ende des Treatments besteht ein signifikanter Unterschied hinsichtlich der Subskala „Psychisches Wohlbefinden“ in beiden Gruppen. Obwohl sich beide Programme hinsichtlich dieser Subskala psychischen Wohlbefindens als effektiv 
erwiesen haben (signifikanter Zeiteffekt), erreichte die Experimentalgruppe am Ende des Treatments signifikant höhere Werte als die Kontrollgruppe. Das psychoedukative Bewegungsprogramm hatte einen größeren Einfluss auf die allgemeine psychische Gesundheit als das Fitnesstraining.

Die SF-36 - Ergebnisse der Katamnese weisen zusammen betrachtet auf eine leichte Verschlechterung der Lebensqualität hin. Dieses wird aus den Subskalen „Allgemeine Gesundheit“ und „Vitalität“ ersichtlich. Beide Gruppen beurteilen ihre Gesundheit einschließlich ihres aktuellen Gesundheitszustands im Zeitraum nach dem Ende der Bewegungsprogramme bis zum Katamnesezeitpunkt negativer (signifikanter Zeiteffekt).

Die Subskalen „Vitalität“, „Körperliche Schmerzen“, „Emotionale Rollenfunktion“ und „Psychisches Wohlbefinden“ zeigen signifikante Unterscheide zwischen den Gruppen. Die drei Subskalen deuten auf einen deutlich verschlechterten Gesundheitszustand bei der Kontrollgruppe im Vergleich zur Experimentalgruppe in diesem Zeitraum hin.

Die körperlichen und psychischen Summenskalen reflektieren den jeweils physischen oder geistigen allgemeinen Gesundheitszustand, wobei wie bei den Subskalen ein höherer Wert einem besseren Zustand entspricht. Die Summenskalenscores werden mit denen der deutschen Normpopulation verglichen. Während der Baselinephase wiesen die Werte der Summenskalen beider Gruppen auf einen im Vergleich $\mathrm{zu}$ der Normstichprobe schlechteren somatischen sowie psychischen Gesundheitszustand hin.

Während der Treatmentphase verbesserte sich die Lebensqualität für beide Gruppen. Die Teilnehmer der Experimentalgruppe erreichten einen Punktwert in der körperlichen wie psychischen Summenskala, der den Summenskalenwert der Normpopulation übertrifft. Die Verbesserungen der Teilnehmer der Kontrollgruppe finden sich in dieser Behandlungsphase ausschließlich bezüglich der somatischen Summenskala. Es gab keine Veränderung bezüglich der psychischen Faktoren. Die Gruppen unterscheiden sich signifikant hinsichtlich ihrer psychischen Lebensqualität. Das psychoedukative Behandlungsprogramm hat somit spezifische Auswirkungen auf die psychische Gesundheit.

In der Phase nach dem Treatment belegen beide Summenskalen einen Rückgang der Gesundheit in beiden Gruppen. Hinsichtlich der körperlichen Variablen ist diese Abnahme der Lebensqualität für beide Gruppen vergleichbar, sie liegen beide wieder 
unter der deutschen Normpopulation, allerdings noch signifikant höher als die bei Beginn der Untersuchung gemessenen Ausgangswerte.

In Bezug auf die psychische Summenskala bleibt die Experimentalgruppe trotz des leichten Rückgangs weiterhin über den Werten der Normpopulation. Der Vergleich mit der Kontrollgruppe zeigt auch hier einen signifikanten Gruppenunterschied.

Dennoch muss berücksichtigt werden, dass die Ausgangswerte der psychischen Summenskala der Experimentalgruppe nicht signifikant über denen der Kontrollgruppe liegen und sich jetzt wenig von denen der Normpopulation unterscheiden. Trotz dieser nicht idealen Ausgangsposition scheint das psychoedukative Bewegungsprogramm einen größeren Einfluss auf die psychischen Faktoren der Lebensqualität als das Fitnessprogramm zu haben, denn die Gruppen unterscheiden sich signifikant. Dieser Effekt ist nachhaltig, da er auch 6 Monate nach Ende der Behandlung zu beobachten ist.

Diese Befunde legen die These nahe, dass die psychologischen Einschränkungen, die mit der Adipositas assoziiert sind, unterschiedlich sein können (Fontaine \& Bartlett, 2003). Während die Experimentalgruppe ein hohes Maß an psychischer Gesundheit, vergleichbar mit der der deutschen Normpopulation, genoss, wies die Kontrollgruppe eine verminderte aus.

Zusammenfassend zeigt sich durch den SF-36, dass die Behandlung mit beiden Bewegungsprogrammen einen deutlich positiven Einfluss auf die gesundheitsbezogene psychophysische Lebensqualität der Teilnehmer hat. Subjektiv- erfahrene, körperliche und psychische Aspekte der Lebensqualität konnten positiv beeinflusst werden.

Das psychoedukative Bewegungsprogramm führte insbesondere $\mathrm{zu}$ einer Verbesserung der sozialen und psychischen Komponenten der Lebensqualität, welche sich auch über den Behandlungszeitraum hinaus bis zum Katamnesezeitpunkt zeigten.

Die Wirkung des Fitnesstrainingsprogramms wurde vorwiegend in den körperlichen Bereichen der Lebensqualität deutlich. Der Vergleich mit den Normwerten der deutschen Bevölkerung zeigt, dass es sich hier um eine spezifische Population handelt, die sich hinsichtlich der körperlichen Bereiche der Gesundheit vom Durchschnitt unterscheidet, d.h. deutliche körperliche Einschränkungen aufweist. In Bezug auf die soziale Funktionsfähigkeit und die psychische Gesundheit ergibt sich dennoch kein einheitliches Bild hinsichtlich der Gesamtstichprobe. 


\subsection{Körperkonzept}

Die Angaben über das Körperkonzept als Teil des Selbstkonzeptes wurden mit den Frankfurter Körperkonzeptskalen (FKKS) nach Deusinger (1998) erhoben. Die FKKS als ein umfassendes Befragungsinstrument (9 Skalen, 64 Items) erfasst wesentliche Aspekte des körperlichen Erlebens und Verhaltens. Durch die sechsstufigen Beantwortungsmöglichkeiten können kleine Veränderungen erfasst werden.

Die Ausgangswerte der Gesamtstichprobe der vorliegenden Untersuchung liegen im Vergleich zu der weiblichen Gesamtstichprobe ( $\mathrm{N}=869)$ von Deusinger (1998) auf acht von neun Körperkonzeptskalen unterhalb der Norm. Die Werte der Skala „Pflege des Körpers“ (SPKF) liegen bei beiden Gruppen über denen der von Deusinger befragten weiblichen Normalpopulation.

Der Vergleich der beiden Gruppen zu diesem Zeitpunkt zeigt einen signifikanten Gruppenunterschied in der Skala „Aspekte der körperlichen Erscheinung“ (SASE). Die Effektstärke ist mit $\eta^{2}=0,11$ als mittel zu bewerten. Während der Treatmentperiode verbesserte sich das Gesamtkörperkonzept der Versuchs- und Kontrollgruppe hoch signifikant. Diese Entwicklung zeigte sich zunächst als ein signifikanter Zeiteffekt auf den Skalen „Gesundheit und körperliches Befinden“ (SGKB), „Körperliche Effizienz“ (SKEF), „Körperkontakt“ (SKKO), „Sexualität“ (SSEX), „Selbstakzeptanz des Körpers“ (SSAK), „Akzeptanz des Körpers durch andere“ (SAKA) und „Aspekte der körperlichen Erscheinung“ (SASE). Bei den restlichen zwei Skalen „Pflege des Körpers und der äußeren Erscheinung“ (SPKF), sowie „Dissimilatorische Prozesse“ (SDIS) ließen sich keine signifikanten Wertverschiebungen beobachten. Es war zu erwarten, dass in Fällen, in denen der Normbereich erreicht bzw. schon überschritten wurde, keine Veränderung zu erwarten waren. Die Veränderungen bzgl. der anderen Subskalen zeigen, dass beide Interventionsformen das Körperbild positiv beeinflussen.

Der Gruppenunterschied in der Skala „Aspekte der körperlichen Erscheinung“ (SASE), der sich schon in der Baseline zeigte, setzt sich in der Behandlungsphase fort.

Hinsichtlich des Körperkontakts (Skala „SKKO“) verbessert sich die Experimentalgruppe signifikant deutlicher als die Kontrollgruppe. Es scheint, dass das psychoedukative Bewegungsprogramm mehr Möglichkeiten bietet, die Einstellungen, d.h. Emotionen, Kognitionen und das Verhalten im Zusammenhang mit zwischenmenschlicher körperlicher Berührung, zu verbessern. Diese Verbesserung 
ist eng mit den signifikanten Verbesserungen in der Skala „Sexualität“ (SSEX) verknüpft. Deusinger (1998) geht von einer engen Assoziation dieser beiden Skalen aus, was auch durch das vorliegende Untersuchungsergebnis gestützt wird.

Alle beschriebenen Ergebnisse lassen sich als ein Erfolg der beiden Bewegungsprogramme werten, der sich zunächst im Gesamtsummenscore aller Skalen für beide Gruppen widerspiegelt. Die Effektstärke über den Zeitraum der Behandlung ist sehr hoch $\left(\eta^{2}=0,442\right)$.

Darüber hinaus weisen der Gruppenunterschieds- und der Interaktionseffekt auf unterschiedliche Wirkungen der beiden Programme hin. Das Körpererleben verbessert sich wesentlich deutlicher durch das psychoedukative Bewegungsprogramm als durch das Fitnessprogramm.

Die Ergebnisse der Katamnese zeigen ein uneinheitliches Bild bezüglich der verschiedenen Subskalen (siehe Tabelle 27 und Abbildung 17). Bei den signifikanten Veränderungen handelt es sich um Gruppenunterschiede, die sich in den Skalen „Gesundheit und körperliches Befinden“ (SGKB), „Pflege des Körpers und der äußeren Erscheinung“ (SPKF), „Sexualität“ (SSEX) und „Aspekte der körperlichen Erscheinung“ (SASE) zeigen. Bezüglich dieser Skalen schneidet die Experimentalgruppe wesentlich besser ab als die Kontrollgruppe, so dass auch der Summenwert der Experimentalgruppe deutlich über dem der Kontrollgruppe liegt. Die Gesamtkörperkonzepte der beiden Gruppen während des Katamnesezeitpunkts sind signifikant unterschiedlich. Die Größenordnung des Effekts liegt bei $\eta^{2}=0,11$ und ist als mittel zu bewerten. Während am Ende des Treatments Tendenzen hinsichtlich dieser Gruppenunterschiede $\mathrm{zu}$ finden waren, scheint das psychoedukative Bewegungsprogramm nachzuwirken, so dass zum Katamnesezeitpunkt ein deutlicher genereller Gruppenunterschied zu vermerken war. Trotz dieses Therapieerfolges liegen am Ende der achtwöchigen Intervention noch sieben von neun Skalenwerte für die Experimentalgruppe und acht von neun für die Kontrollgruppe unter denen der Normalpopulation. Während des Zeitraums der Katamnese sind es noch fünf von neun für die Experimentalgruppe und acht von neun für die Kontrollgruppe. Das Körperkonzept ist somit bei Ende des Programms und des Katamnesezeitraums in beiden Gruppen noch auffällig.

Diese Ergebnisse stützen die Aussage, dass Menschen mit klinisch manifester Adipositas häufig unter einer Körperschema- oder Körperbildstörung leiden (vgl. 
Kap. 2.9). Nach Deusinger (1998) ist das „Gesamt-Körperkonzept“ adipöser Personen weniger ,positiv“ als das „,normalgewichtiger“ Personen. Eine psychische Beeinträchtigung der Menschen mit Adipositas wird zusätzlich durch die soziale Diskriminierung begünstigt.

Die Verbesserungen der eng assoziierten Körperkonzepte „Gesundheit und körperliches Befinden“ (SGKB) und „Körperliche Effizienz“ (SKEF) können vor dem Hintergrund der Koordination, Kraft und aeroben Leistungsfähigkeit gemessenen Effekte (vgl. Kap. 7.7 - 7.9) erklärt werden.

Die Verbesserungen des Körperkonzepts „Aspekte der körperlichen Erscheinung“ (SASE) für beide Gruppen sind schwer interpretierbar, da sich die Gruppen am Anfang der Studie signifikant unterschieden haben.

Das signifikant positivere Gesamtkörperkonzept der Experimentalgruppe im Vergleich zu dem der Kontrollgruppe legt die Vermutung nahe, dass das psychoedukative Bewegungsprogramm eine positivere Auswirkung auf das Gesamtkörperkonzept hat als das Fitnessprogramm. Die Teilnehmer der Experimentalgruppe schreiben sich eine größere körperliche Effizienz zu und verfügen über eine bessere Selbstakzeptanz dem eigenen Körper gegenüber. Zudem haben sie ein verstärktes Gesundheitsbewusstsein, welches sich durch ein entsprechendes Gesundheitsverhalten äußert. Inwieweit sich diese Veränderung des Körperkonzeptes auch auf weitere Selbstkonzepte auswirken kann, muss an anderer Stelle entsprechend untersucht werden.

In der Auswertung des FKKS lassen sich die einzelnen Skalenwerte in positive, neutrale und negative Körperkonzepte einordnen (siehe Kap. 6.3, Abbildung 17). Nach Deusinger (1998) weisen negative Körperkonzepte auf bedeutsame psychische Probleme und Störungen hin.

Zu Beginn der Bewegungsprogramme lagen für die Kontrollgruppe zwei Skalenwerte im negativen Bereich, zwei im positiven und die restlichen fünf im neutralen Bereich. Hingegen war bei der Experimentalgruppe ein Skalenwert im negativen Bereich, drei waren im positiven und die restlichen fünf im neutralen Bereich.

Die Skalen, deren Werte sich schon zu Behandlungsbeginn im positiven Bereich befanden, decken sich bei beiden Gruppen. Diese zwei Skalen „Pflege des Körpers und der äußeren Erscheinung unter Beachtung der Funktionsfähigkeit“" (SPKF) sowie „Dissimilatorische Prozesse“ (SDIS) verbessern sich weiter signifikant. Auch die 
Skalen, deren Werte im negativen Bereich waren bzw. grenzwertig zum neutralen Bereich lagen, sind für beide Gruppen die gleichen. Es sind die Skalen „Selbstakzeptanz des Körpers“ (SSAK) und „Akzeptanz des Körpers durch andere“ (SAKA) und beide zeigten eine hoch signifikante Werteverbesserung bis in den neutralen Bereich.

Beide Skalen, die die Hauptproblematik der Körperbildtherapie bei Adipositas repräsentieren, hielten sich auch sechs Monate nach Ende des Treatments im unteren neutralen Bereich. Die Skalenwerte der Kontrollgruppe, die zu Beginn in den neutralen Bereich eingeordnet werden konnten, veränderten sich signifikant, blieben aber mit Ausnahme der Skala „Körperkontakt“ (SKKO), die in den positiven Bereich übertrat, im neutralen Bereich. Drei der fünf Skalenwerte der Experimentalgruppe aus dem neutralen Bereich veränderten sich signifikant und wechselten in den positiven Bereich. Die übrigen zwei blieben trotz Verbesserung im oberen neutralen Bereich.

Zusammenfassend zeigen die Ergebnisse der Studie, dass sich die Körperkonzepte von Menschen mit Adipositas unter dem Einfluss bewegungstherapeutischer Interventionen signifikant verbessern. Das psychoedukative Bewegungsprogramm hatte einen stärkeren und länger andauernden Einfluss auf die Aspekte des körperlichen Erlebens als das Fitnessprogramm. Dadurch profitierten die Teilnehmer der Experimentalgruppe mehr als die Teilnehmer der Kontrollgruppe von der jeweiligen bewegungstherapeutischen Intervention.

\subsection{Depressivität}

Im folgenden Abschnitt wird der Frage nachgegangen, welche Effekte die Bewegungsprogramme auf die Stimmung der Teilnehmer haben. Bei der Interpretation der Ergebnisse muss berücksichtigt werden, dass für den Summenscore alle 21 Items des Beck Depression Inventars berechnet wurden. Das Miteinbeziehen des 19. Items „Gewichtsverlust“ erweist sich als problematisch und führt bei der vorliegenden Stichprobe zu einer Überschätzung der Depressivität (vgl. Kap. 5.3.6). Allerdings zeigte ein Vergleich der dargestellten Ergebnisse mit den Ergebnissen einer Berechung ohne das Item 19 nur unwesentliche Unterschiede, die insbesondere die Signifikanz der Ergebnisse nicht veränderte. 
Die durchschnittliche Depressivität der Experimentalgruppe war während der Baselineperiode unauffällig (BDI-Wert <11). Die Ausgangswerte der Kontrollgruppe lagen zum Zeitpunkt des Treatmentbeginns im Grenzbereich zur Depression (BDIWert $=11,54)$.

Nach den Bewegungsprogrammen sanken die Summenwerte des BDI für beide Gruppen signifikant. Bei $\eta^{2}$ von 0,193 ist dieses als ein großer Effekt zu bewerten. Der Rückgang der BDI-Scores ist für die Experimentalgruppe wesentlich größer als für die Kontrollgruppe. Auch die Verbesserung in der Kontrollgruppe ist erwähnenswert, da nach dem Fitnessprogramm die BDI-Werte als unauffällig anzusehen ist. Im Follow-up-Zeitraum bleiben beide Gruppen trotz eines leichten Anstiegs der BDI-Scores in diesem unauffälligen Bereich. Die Gruppen liegen beide in der Kategorie „unauffällig“, wobei sie sich dennoch signifikant voneinander unterscheiden. Die Effektstärke mit $\eta^{2}=0,082$ zeigt einen mittleren Effekt.

Betrachtet man die gesamte Untersuchungszeit, kam es zu einer Normalisierung bzw. weiteren Verbesserung der Stimmungslage für beide Gruppen. Diese blieb über den Nachbeobachtungszeitraum stabil. Während die Experimentalgruppe zu keinem Messzeitpunkt eine Depressivität aufwies und ihre Stimmungslage noch deutlich verbesserte, profitierten insbesondere die Teilnehmer der Kontrollgruppe, die vor dem Fitnessprogramm in mäßigem Maß von Depressivität betroffen waren.

Die Ergebnisse bestätigen die These, dass gezielte körperliche Aktivität eine positive Wirkung auf die Depressivität besitzt.

Der Vergleich der untersuchten Gruppen mit der Normstichprobe (Hautzinger et al., 1995) zeigt, dass die Werte in der Baselinephase deutlich über den Werten der gesunden Normstichprobe liegen. Die Ergebnisse der vorliegenden Studie unterscheiden sich allerdings nicht wesentlich von den Befunden in der Literatur beschriebener klinischer Stichproben (Herpertz \& Saller, 2001; Telch \& Agras, 1994).

Durch Verbesserungen der Depressivität innerhalb der bewegungstherapeutischen Gruppen werden die mittleren Summenwerte mit den Werten der gesunden Normstichprobe, angegeben im Manual des BDIs, vergleichbar. Im Falle der Experimentalgruppe übertrifft der Mittelwert sogar den der gesunden Normstichprobe. Dieser Effekt ist auch nach dem Zeitraum der Katamnese zu finden.

Die Kategorisierung der Gruppen nach dem Ausmaß an Depressivität zeigt eine gewisse Heterogenität innerhalb beider Gruppen. Es handelt sich um eine Stichprobe, 
bei der ein kleiner Anteil von weiblichen Teilnehmern eine klinisch relevante Ausprägung der Depressivität zeigt. Dieses Ergebnis entspricht vorigen Beobachtungen, bei denen Menschen mit Adipositas hinsichtlich ihrer psychischen Funktionsfähigkeit heterogen waren und adipöse Frauen anfälliger für eine Depression als Männer sind (vgl. Kap. 2.8).

Während der gesamten Untersuchungszeit gab es bei einigen Teilnehmern Verschiebungen und häufige Kategorienwechsel; in der Experimentalgruppe seltener als in der Kontrollgruppe. Bemerkenswert ist auch die Häufigkeit des Vorkommens einer klinisch relevanten Ausprägung sechs Monate nach dem Ende des Treatments in der Kontrollgruppe.

Zusammenfassend zeigen die Ergebnisse der Studie, dass sich die Stimmungslage von Menschen mit Adipositas unter dem Einfluss bewegungstherapeutischer Interventionen signifikant verbessert. Das psychoedukative Bewegungsprogramm hatte einen größeren und länger andauernden Einfluss auf die Depressivität als das Fitnessprogramm. Die Teilnehmer der Experimentalgruppe profitieren mehr als die Teilnehmer der Kontrollgruppe.

Es gibt innerhalb der untersuchten Gesamtstichprobe eine Untergruppe von Frauen, die stark von einer depressiven Symptomatik betroffen sind.

\subsection{Essstörungssymptomatik}

Die Anwendung des strukturierten Inventars für Anorektische und Bulimische Essstörung (SIAB) wurde als diagnostisches Instrument genutzt. Geprüft wurde, ob es Studienteilnehmer gab, die das Vollbild Binge Eating Disorder (BED) nach DSMIV erfüllten. Weiterhin wurde überprüft, welchen Einfluss die bewegungstherapeutische Behandlung auf die BED hat. Die Ergebnisse zeigen, dass sieben Teilnehmerinnen (drei aus der EG und vier aus der $\mathrm{KG}$ d.h., $12 \%$ der Gesamtstichprobe) zu Beginn der Untersuchung die Kriterien einer BED erfüllen. Bei Beginn der Behandlung sind es fünf (drei aus der EG und zwei aus der KG, d.h. $9 \%$ der Gesamtstichprobe). Die beiden Gruppen sind zu diesen Zeitpunkten vergleichbar. Nach Ende der Behandlung und über den Nachbeobachtungszeitraum bleiben 4 Teilnehmerinnen mit einer BED, alle aus der Kontrollgruppe.

Die Tatsache, dass drei Teilnehmerinnen der Experimentalgruppe nach der Behandlung ihre BED-Symptomatik nicht mehr zeigen, lässt die Vermutung zu, dass 
das psychoedukative Bewegungsprogramm einen positiven Einfluss auf die Heißhungerattacken hat. Die Ergebnisse der Kontrollgruppe sind schwer interpretierbar, da insgesamt fünf Teilnehmerinnen zu verschieden Messzeitpunkten ein gestörtes Essverhalten zeigten. Zwei Teilnehmerinnen erfüllen über die gesamte Studienzeit die wissenschaftlichen Kriterien einer BED. Eine dritte Probandin zeigt eine BED-Symptomatik zu drei von den vier Messzeitpunkten, die vierte Teilnehmerin am Anfang und am Ende des gesamten Untersuchungszeitraums und die fünfte Teilnehmerin nur einmal am Ende des Treatments. Diese Ergebnisse zeigen, dass das untersuchte Fitnessprogramm keinen Einfluss auf eine Binge Eating Disorder hatte. Der Prozentsatz von 7-12 \% der Teilnehmer mit einer BED in dieser Studie unterscheidet sich deutlich von den Prävalenzdaten in der Literatur. In klinischen Populationen, die mit dem Ziel einer Gewichtsreduktion ärztliche oder psychologische Hilfe aufsuchen, finden sich ca. $30 \%$, die die erforderlichen Kriterien einer BED erfüllen (de Zwaan, 2002). Die These, dass BED mit einer höheren Komorbidität anderer psychischer Störungen (insbesondere einer höheren depressiven Symptomatik) einhergeht, kann durch die Datenlage der vorliegenden Studie partiell bestätigt werden (Stunkard, 2004). Etwa die Hälfte der Teilnehmerinnen mit einer BED Symptomatik wiesen Werte einer mäßigen bzw. klinisch relevanten Depressivität auf.

Obwohl es sich bei dem psychoedukativen Bewegungsprogramm um kein störungsspezifisches Behandlungsprogramm für Personen mit Binge Eating Disorder handelt, zeigt sich zusammenfassend, dass es hinsichtlich dieses essstörungsbezogenen Parameters einen positiven Einfluss gibt.

\subsection{Subjektive Einschätzung der Wirkfaktoren der Bewegungsprogramme durch die Teilnehmer}

Die Wirkfaktoren der Bewegungsprogramme wurden mit dem DFBT von Hölter (2004) nach der Neustrukturierung von Ungerer-Röhrig (2001) gemessen. Der Einfluss der beiden bewegungstherapeutischen Programme auf die Erfahrungen und das Erleben der Teilnehmer wurde einmalig nach Beendigung der therapeutischen Beeinflussung untersucht. Die Erfassung schloss folgende 4 Faktoren ein: a) biographische Erfahrungen; b) Körper- und Selbsterfahrung; c) Erleben von Gruppenbeziehungen und d) Erleben von Bewegung und Wohlbefinden. So erhielt man 
Aufschluss darüber, als wie wirksam die bewegungstherapeutischen Interventionen empfunden wurden.

Der DFBT ist z. Zt. das einzige Instrument, welches die Wirkungsweise bewegungsund sporttherapeutischer Interventionen hinsichtlich psychologischer Parameter misst, da das Instrument besonders dann geeignet ist, wenn die bewegungstherapeutische Intervention eine psychotherapeutische Zielsetzung aufweist. Durch die sechsstufige Beantwortungsskala lassen sich auch kleinere Veränderungen erfassen.

Die Teilnehmer beider Gruppen bewerteten die durch verschiedene Subskalen abgefragten Aspekte bewegungstherapeutischer Praxis unterschiedlich. So wurde der Faktor „Biographische Erfahrung“ von beiden Gruppen mit „trifft eher nicht zu“ am niedrigsten bewertet. Dies überrascht wenig, da keines der beiden Programme eine auf das biographische Wiedererleben gerichtete Zielsetzung oder entsprechende Inhalte transportiert.

Hinsichtlich der Faktoren „Erfahrungen von Körper und Selbst“ wie auch „Erleben von Beziehungen“ war die Bewertung mit „trifft eher zu“ wesentlich höher. Obwohl es keinen signifikanten Unterschied bezüglich dieser beiden Faktoren zwischen den beiden Gruppen gab, lagen die Werte der Experimentalgruppe höher als die der Kontrollgruppe.

Dieses lässt sich auf die Inhalte des psychoedukativen Bewegungsprogramms zurückführen, da die Körpererfahrung wie auch die Selbsterfahrung durch die Bewegung zentrale Themen des Konzepts sind. Auch der Aspekt der Gruppenbeziehung konnte durch Gruppen fördernde Gestaltung der edukativ ausgerichteten Therapieeinheiten der Experimentalgruppe verstärkt werden. Es erstaunt jedoch, dass eine „eindimensionale Intervention“ wie das Fitnesstraining hinsichtlich der o. g. zwei Parameter vergleichbare Ergebnisse erzielte.

Der Faktor „Erleben von Bewegung und Wohlbefinden“ erhielt von beiden Gruppen mit „trifft zu“ die höchste Bewertung. Beide Interventionen waren hinsichtlich dieses Faktors am wirksamsten. Aber auch hier scheint die Experimentalgruppe einen Vorsprung zu haben, wie ein signifikanter Gruppenunterschied zeigt. Die Größenordnung des Effekts ist mit $\eta^{2}=0,155$ als groß zu bewerten.

Die positive Bewertung des letzten Faktors durch beide Gruppen deckt sich weitgehend mit den Inhalten der bewegungstherapeutischen Programme. Es wird angenommen, dass Freude und Spaß an der Bewegung ein zentrales Ziel jeder 
bewegungs- und sporttherapeutischen Maßnahme bei der Adipositastherapie ist (Wirth, 2003; Wing \& Jakicic, 2000; Brownell, 1995). Für die intrinsische Motivation und die mit ihr verbundenen Konsequenzen (vgl. Kap. 4.9.8.3) ist es insbesondere wichtig, Menschen mit Adipositas an die Bewegung so heranzuführen, dass positive Erfahrungen durch die Bewegung vermittelt werden.

Die Tatsache, dass das psychoedukative Bewegungsprogramm besser bewertet wurde, lässt sich auch darauf zurückführen, dass ein besonderer Schwerpunkt auf den Faktor „Wohlbefinden“, vermittelt durch verschiedene Entspannungsmöglichkeiten, gelegt wurde.

Ein Vergleich mit ähnlichen Populationen ist zum jetzigen Zeitpunkt nicht möglich, da die Datenbasis fehlt. Die Ergebnisse von Studien, in denen der DFBT angewendet wurde (Deimel, 2001; Alexandridis, 2005), lassen sich auf Grund der unterschiedlichen Krankheitsbilder nicht direkt vergleichen.

Zusammenfassend kann gesagt werden, dass beide Bewegungsprogramme hinsichtlich ihrer Wirksamkeit positiv eingeschätzt wurden. Teilnehmer empfanden insbesondere die Gruppen- und Körpererfahrungen positiv und erlebten ein starkes Gefühl von Wohlbefinden durch die Bewegung.

\subsection{Allgemeine Ausdauerleistungsfähigkeit gemessen durch fahrradergo- metrische Belastungsuntersuchung und Walkingtest}

Die allgemeine Ausdauerleistungsfähigkeit wurde in einer fahrradergometrischen Belastungsuntersuchung nach dem WHO-Schema und durch den Walkingtest ermittelt. Der fahrradergometrische Test ist durch eine exakte Belastungsdosierung und Reproduzierbarkeit gekennzeichnet. Das Testschema ist standardisiert. Im Bereich der Adipositas ist die gewichtsentlastende Funktion des Fahrrads von großer Bedeutung.

Es werden große Teile der gesamten Muskulatur beansprucht, aber dennoch weniger als beim Gehen. Einige Probanden können ihre submaximale, kardiopulmonale Ausbelastung jedoch aufgrund mangelnder Beinkraft nicht erreichen.

Die Herzfrequenzen wurden für die einzelnen Belastungsstufen über ein Polar Herzfrequenz-Messgerät registriert. Sie wurden zur Berechnung der „Power Work Capacity“ (PCW) und zur Beurteilung der Leistungsfähigkeit im Vergleich mit der Norm herangezogen. Eine noch genauere Bestimmung der Leistungsfähigkeit durch 
Messung der Laktatwerte auf den einzelnen Belastungsstufen konnte aus organisatorischen Gründen nicht durchgeführt werden.

Die Ergebnisse der fahrradergometrischen Untersuchung zeigen, über die Gesamtstudienzeit betrachtet, keine signifikanten Unterschiede zwischen den beiden Gruppen (vgl. Kap. 6.7).

Als Erklärung für die leichte Verbesserung in beiden Gruppen während der Baselineperiode wird angenommen, dass diese durch einen Wiederholungseffekt entstanden ist. Mögliche Faktoren könnten sein, dass die Testsituation in dem EKGRaum der Klinik den Probanden beim zweiten Mal vertrauter war und dass kurz vor dem Beginn des Bewegungsprogramms eine höhere Motivation bestand.

Die Effekte nach der Behandlungsperiode waren für beide Gruppen vergleichbar. Die Ausdauerfähigkeit der Probanden verbesserte sich signifikant, sowohl absolut betrachtet als auch relativ, d.h. aufs Körpergewicht bezogen (siehe Tabelle 35 und Abbildung 21). Die Effektstärke, die für die absolut geleistete Wattanzahl mit $\eta^{2}=0,301$ berechnet wurde, gilt als ein großer Effekt. Das Ausmaß dieser Verbesserungen zeigt, dass beide Behandlungsprogramme ähnlich positiv auf die aerobe Ausdauer wirken und keine der beiden Interventionen der anderen bzgl. der Ausdauerleistung überlegen ist.

Dieses Ergebnis entspricht den Ergebnissen anderer Studien, die eine Steigerung der geleisteten Wattanzahl nach bewegungstherapeutischen Interventionen gemessen haben (Völker, Lagerstrøm, \& Matheis, 1985; Duperly, 1998). Die Messungen nach der Follow-up-Periode zeigen eine nachhaltige Wirkung beider Programme. Der beobachtete leichte Werterückgang ist nicht signifikant, das durch die Programme erreichte Fitnessniveau zeigte sich auch sechs Monate nach der Interventionszeit.

Trotz der o.g. positiven Interventionseffekte wiesen Probanden mit Adipositas zweiten Grades und Probanden mit extremem Übergewicht in einem deutlicheren Maße eine starke Einschränkung der Leistungsfähigkeit auf, wenn die Ergebnisse in Relation zu den von Rost, Heck und Hollmann, (ohne Jahrangabe) genannten Sollleistungen gesetzt werden.

Die zusätzliche Anwendung des 2-km-Walkingtests dient der Bestimmung der walkingspezifischen Leistungsfähigkeit, die ein umfassendes Bild hinsichtlich der körperlichen Ausdauerfähigkeit der Teilnehmer darstellt. Die zur Interpretation herangezogenen Werte sind Erfahrungswerte, die aus der Studie zur Entwicklung des 
Tests gewonnen wurden. In der vorliegenden Studie gab es zunächst Teilnehmer, die aus medizinischen Gründen (Gelenkprobleme, Schmerzen, akuter Darminfekt, Schwangerschaft etc.) nicht am Walkingtest teilnehmen konnten. Insgesamt konnten über die Gesamtstudienzeit 7 Fälle nicht in die Auswertungen mit einbezogen werden.

Des Weiteren zeigte sich, dass bei den Berechnungen des Fitnessindexes die im Manual des Tests angegebene Formel für die Teilnehmer mit einem extremen Übergewicht (BMI 44,7-55,7) ungeeignet war, da negative Zahlen entstanden, die nicht mit den positiven vergleichbar waren. Der Grund für das Entstehen der negativen Werte bei der Anwendung der Formel liegt zum Teil an den hohen BMIWerten und teilweise an der deutlich längeren Walkingzeit (vgl. Berechnung des Walking-Leistungsindex, Kap. 5.3.10). So mussten weitere 4 Probanden (1 Experimentalgruppe/3 Kontrollgruppe) aus der Bewertung ausgeschlossen werden. Eine weitere Besonderheit bezüglich der Interpretation der Walkingtestergebnisse ist die Tatsache, dass es in der Baselineperiode eine signifikante Verbesserung der Walkingzeit bzw. des Leistungsindex in beiden Gruppen gab. Die Effektstärke ist mit $\eta^{2}=0,276$ groß. Das Phänomen der gesteigerten Fitness ohne Intervention zeigt sich beim Walkingtest stärker als bei der Fahrradergometrie. Es wird berichtet, dass die Walkingstrecke beim ersten Test häufig zu langsam gegangen wird. Die Probanden neigen dazu sich $\mathrm{zu}$ unterschätzen. Um ein Gefühl für die individuelle Maximalgeschwindigkeit zu gewinnen wird empfohlen, diese vorher auszuprobieren (Bös, 1994). Außerdem wird bei Probanden mit Adipositas vermutet, dass psychologische Parameter wie Aufregung, Angst, Unsicherheit wegen der unbekannten und schwer einschätzbaren Strecke eine entscheidende Rolle spielen.

Durch die Behandlung kam es zu weiteren deutlichen Veränderungen. Die Interventionseffekte zeigen einen signifikanten Unterschied hinsichtlich des Faktors Zeit und des Faktors Gruppe. Die Verbesserung der Gehleistung bezogen auf beide Gruppen hat die Effektstärke von $\eta^{2}=0,449$ und ist somit als ein großer Effekt zu werten.

Der Gruppenunterschied deutet darauf hin, dass die Experimentalgruppe durch das psychoedukative Bewegungsprogramm mehr profitiert als die Kontrollgruppe. Die Berechnung der Effektstärke mit $\eta^{2}=0,124$ weist auf einen mittleren Effekt hin. Der Werterückgang sechs Monate nach der Behandlungszeit ist signifikant für beide Gruppen. Die Leistungsfähigkeit nimmt ab, bleibt aber in beiden Gruppen noch 
höher als vor dem Treatment. Hier handelt es sich um einen mittleren Effekt $\left(\eta^{2}=0,095\right)$.

Zusätzlich findet sich ein signifikanter Gruppenunterschied. Die Ausdauerleistung der Kontrollgruppe reduziert sich signifikant deutlicher als die der Experimentalgruppe. Auch dieser Effekt ist bei $\eta^{2}=0,091$ von einer mittleren Größenordnung.

Es fällt auf, dass die Ergebnisse von fahrradergometrischer Belastungsuntersuchung und Walkingtest hinsichtlich des Untersuchungsverlaufs vergleichbar sind. Das psychoedukative Bewegungsprogramm hat auch auf die walkingspezifische Leistungsfähigkeit einen höheren Einfluss und das über den Behandlungszeitraum hinaus.

Die Bedeutung von Walking als einer alltagsnahen, einfach erlernbaren, unaufwendigen Trainingsform für die Behandlung der Adipositas nahm in den letzten Jahren zu. Das Walking (Nordic Walking) wird sehr häufig als alleinige bewegungstherapeutische Intervention oder im Rahmen von multimodalen Gewichtsreduktionsprogrammen eingesetzt und in seiner Wirksamkeit erforscht (Fogelholm et al., 2006). Walking als therapeutischer Inhalt war mit 4 Terminen in beide Interventionen der vorliegenden Studie integriert. Es wird angenommen, dass dieses Training, welches der Testsituation sehr ähnlich ist, zur Steigerung der Leistungsfähigkeit beigetragen hat.

Der Vergleich mit Normwerten verdeutlicht jedoch, dass, obwohl ähnlich wie bei der fahrradergometrischen Untersuchung positive Effekte in Bezug auf die Ausdauerleistungsfähigkeit der Teilnehmer erreicht wurden, diese bei der untersuchten Stichprobe im Vergleich zu der Norm immer noch als niedrig zu bewerten sind.

Ordnet man die gemessenen Ergebnisse der walkingspezifischen Ausdauerleistungsfähigkeit in die Leistungskategorien des Walkingtests ein, so zeigt sich, dass die Teilnehmer der vorliegenden Stichprobe als „sehr schwach“ eingestuft werden (Bös et al., 1992). Trotz der niedrigen Klassifizierung bezüglich beider Testverfahren ist die erreichte verbesserte Leistungsfähigkeit angesichts der gesundheitlichen Auswirkungen sehr bedeutsam. Nach Blair und Leermakers (2004) besteht bei verbesserter körperlicher Leistungsfähigkeit sowohl für normalgewichtige als auch für übergewichtige Männer und Frauen eine niedrigere Mortalität. Diese Ergebnisse werden durch Untersuchungen von Blair \& Brodney (1999) und Paffenbarger et al., (1993) bestätigt. 
Zusammenfassend verdeutlichen die Ergebnisse dieser Untersuchung die Wirksamkeit bewegungstherapeutischer Programme auf das Herz-Kreislauf-System bzw. auf die körperliche Ausdauerleistungsfähigkeit bei Menschen mit Adipositas. Dieser Leistungszuwachs ist auch nach der Katamnesezeit $\mathrm{zu}$ finden. Keines der beiden bewegungstherapeutischen Programme zeigte sich dem anderen bezüglich der Ausdauerleistung deutlich überlegen. Das psychoedukative Bewegungsprogramm scheint jedoch im Bezug auf die walkingspezifische Ausdauerleistungsfähigkeit effektiver zu sein.

\subsection{Koordinative Fähigkeiten}

Die besondere Bedeutung der Koordination für Menschen mit Adipositas ergibt sich aus der Tatsache, dass die Körperfülle die Motorik erschwert. Motorische Einschränkungen entstehen nicht ausschließlich aufgrund einer mangelnden Ausdauerleistungsfähigkeit, sondern auch aufgrund der Koordination. Die Koordination sollte gefördert werden, um durch ökonomisch und richtig ausgeführte Bewegungen die Belastung des Körpers, der ohnehin durch das Übergewicht vorbelastet ist, gering zu halten.

Die Koordinationsfähigkeit wurde mittels des BKT-Kur von Bös et al. (1992) gemessen. Die Testaufgaben beinhalten die koordinativen Anteile motorischer Leistungsfähigkeit und schließen konditionelle Anforderungen fast vollständig aus. Die Testübungen sollen Störungen und Schwächen im Bereich der koordinativen Fähigkeiten aufdecken (Wydra, 2004).

Kritisch anzumerken ist die dichotome Beurteilung der Testaufgaben in gelöst bzw. ungelöst. Es können keine Abstufungen für eine Aufgabe vorgenommen werden. Angesichts der Spannweite bei der Ausführung in der Praxis erscheint eine differenziertere Beurteilung jedoch angebracht. Bös et al. (1992) weisen zwar darauf hin, dass bei der Ergebnisinterpretation ,auch qualitative Aspekte der Motorik berücksichtigt werden“ (S. 160) sollen, genauere Angaben machen sie aber nicht. Manche Testübungen eignen sich aufgrund der anatomischen Besonderheiten der Testpopulation (BMI $\geq 35$ ) nicht und sollten überdacht werden.

Menschen mit Übergewicht sind unsicher hinsichtlich ihres Gleichgewichts, sie haben insbesondere Angst vor dem Fallen. So sind die Gleichgewichtsaufgaben auf 
dem Balken, „Balancieren rückwärts“, „Ballprellen“ für sie mit einer besonderen Unsicherheit und Stürzgefahr verbunden.

Testaufgaben wie „Hampelmann“ und „Hopserlauf“ beinhalten aufgrund des Springens für einige der Testpopulation eine Fehl- bzw. Überbelastung und stellen ein Verletzungsrisiko dar. Das Springen ist wegen des Übergewichts an sich und wegen der mit Adipositas assoziierten orthopädischen Gelenkbeschwerden eine wenig geeignete Bewegungsform.

Die Testaufgabe „Ball umgreifen“ ist für einige Teilnehmer aufgrund des großen Bauch- und Oberschenkelumfangs besonders schwer oder gar nicht ausführbar. Da zur Überprüfung der Koordination bei Erwachsenen der BKT-Kur momentan der einzige veröffentlichte standardisierte Test ist, erschien sein Einsatz in der vorliegenden Untersuchung trotz der Einschränkungen die optimale Lösung zu sein.

Betrachtet man die Koordinationsfähigkeit über die Gesamtstudienzeit an allen Messzeitpunkten bei beiden Gruppen, sieht man einen fast parallelen Verlauf. Bezüglich der Koordinationsfähigkeit zeigen sich keine signifikanten Gruppenunterschiede.

Die koordinativen Fähigkeiten beider Gruppen liegen bei Beginn der Behandlung trotz eines leichten Anstiegs vor der Intervention unter Berücksichtigung des Alters und des Geschlechts im unteren, d.h. schwachen Bereich. Dies entspricht den Beobachtungen von Ehrhardt und Seeck (1990), nach denen bei ,inaktiven Adipösen weniger gut ausgeprägte koordinative Fähigkeiten vorkommen“" (S. 1150). Die leicht verbesserten Werte zu Beginn der Therapie sind signifikant. Die Effektstärke ist mit $\eta^{2}=0,244$ als groß zu bewerten.

Das Phänomen der Verbesserung ohne Intervention in der Baseline-Periode lässt sich ähnlich wie beim Walkingtest durch einen Wiederholungseffekt erklären. Beim zweiten Mal sind Testsituation und Testaufgaben bekannter und vertrauter.

Durch die bewegungstherapeutischen Maßnahmen verbesserte sich die Koordinationsfähigkeit hochsignifikant (siehe Tabelle 39, Abbildung 24). Die Effektstärke liegt bei $\eta^{2}=0,546$, das heißt, es handelt sich um eine beachtliche Verbesserung.

Die Summenwerte entsprechen im Mittel bezogen auf Alter und Geschlecht dem unteren „mittleren Koordinationsbereich“. Bös et al. (1992) bezeichnen eine sehr gute bis mittlere Testbeurteilung als „normale“ Koordination, die keiner „speziellen Fördermaßnahmen zur Verbesserung der Koordination“ (S. 160) bedarf. Hübschmann (1997) und Kloos (1993) konnten bei ihren Probandinnen nach einem 
Bewegungsprogramm nur tendenzielle Verbesserungen der Koordination feststellen. Allerdings lagen in dieser Untersuchung die Ausgangswerte bereits im mittleren Normbereich. Bei einem schwachen Ausgangsniveau, wie es bei der vorliegenden Untersuchung der Fall ist, lassen sich in kurzer Zeit deutlichere Verbesserungen erzielen.

Die Ergebnisse der vorliegenden Studie fielen deutlicher aus als die der Studie von Duperly (1998). Auch Duperly stellte signifikante Verbesserungen der Koordinationsfähigkeit bei extremer Adipositas nach einem achtwöchigen Trainingsprogramm fest. Die Tatsache, dass die Teilnehmer der vorliegenden Studie eine bessere Entwicklung zeigen, könnte auf die beiden Bewegungsprogramme zurückgeführt werden. Obwohl innerhalb keiner der beiden Interventionen ein explizites Koordinationstraining durchgeführt wurde, waren die Bewegungsaufgaben vielseitig. In der Katamnesezeit bleiben die Effekte bezüglich der Koordination im Allgemeinen recht gut erhalten. Beide Behandlungsansätze hatten eine ähnliche Auswirkung auf die Ausdauerleistungsfähigkeit, eine kleine Tendenz zu einer besseren Wirksamkeit lässt sich zugunsten der Experimentalgruppe verzeichnen. Sie verbesserte sich leicht, während bei der Kontrollgruppe ein minimaler Wertrückgang gemessen wurde.

Diese deutlichen Ergebnisse weisen darauf hin, dass keine neurologischen Schädigungen bei den Probanden vorlagen. Die schwache Koordination kann auf ,sozial bedingte motorische Fehlentwicklungen in der frühen Kindheit, im Sportunterricht der Schule“ (Bös et al., 1992, S. 151) zurückgeführt werden. Hinzu kommt, dass die Verbindung von Inaktivität und Übergewicht an sich eine wichtige Rolle spielt.

Die hochsignifikante Verbesserung der Koordination durch das bewegungstherapeutische Programm kann bedeutsame, positive Auswirkungen auf das Bewegungsverhalten der Probanden mit Adipositas haben.

„Ein Koordinationstraining hat dabei immer positive Auswirkungen auf andere Dimensionen der Leistungsfähigkeit“ (Bös et al., 1992, S. 48). Die Koordination ermöglicht erst das Erlernen bestimmter Techniken für alle denkbaren Sportarten. Auch geringfügige Störungen der Koordination verhindern die harmonische, effektive und ökonomische Ausführung von Bewegungshandlungen in Sport und Alltag (Beruf). Auf der anderen Seite können durch schwere Koordinationsstörungen einschneidende Einschränkungen entstehen, die die Lebensqualität erheblich mindern können (Bös et al., 1992). 
Verbesserungen der koordinativen Fähigkeiten bieten nach Ehrhardt und Seeck (1990) „ . . . bei richtiger Übungsauswahl die Chance, über schnelle Lernfortschritte und ein verbessertes Bewegungsverhalten Erfolgserlebnisse, Bestätigung, Bewegungsfreude und eine überdauernde Motivation für Bewegung zu schaffen bzw. zu stärken“ (S. 1151).

Diese Zusammenhänge zeigen die Bedeutung von koordinationsverbessernden Maßnahmen gerade auch in der Adipositasbehandlung auf.

Zusammenfassend verdeutlichen die Ergebnisse, dass bei Menschen mit Adipositas in der Regel mäßige bis geringe Einschränkungen der koordinativen Fähigkeiten vorliegen, die durch bewegungstherapeutische Interventionen in ihrem Ausprägungsgrad verbessert werden konnten. Der Vergleich der beiden Interventionsprogramme zeigt, dass beide Behandlungsformen bezüglich der Koordination ähnlich effizient sind.

\subsection{Kraft}

Die Kraft als motorische Fähigkeit bei Menschen mit Adipositas soll insbesondere unter zwei Gesichtspunkten betrachtet werden. Sie wird zum einen durch den Bewegungsmangel und zum anderen durch indikations- bzw. störungsspezifische Aspekte, d.h. durch das ungünstige Verhältnis von Körpergewicht und Muskelmasse, geprägt (Ehrhardt \& Seeck, 1990).

Die Bedeutung des Krafttrainings als einer Trainingsform für die Behandlung der Adipositas liegt neben den metabolischen Effekten darin, eine Verbesserung der o.g. Relation zu erzielen. Der ohnehin in Mitleidenschaft gezogene Bewegungs- und Stützapparat der Adipösen soll geschützt werden (vgl. Kap. 4.9.2)

Durch den Krafttest konnte ein umfassendes Bild bzgl. des Fitnesslevels und der körperlichen Leistungsfähigkeit der Teilnehmer gewonnen werden. Ein sportpraktischer Krafttest mit drei Testübungen wurde zur Beurteilung der Kraftfähigkeit ausgewählt. Die Testaufgaben sollen den Verlauf von Funktions- und Leistungsschwächen, insbesondere im Bereich der Rumpfbeugemuskulatur, Schulterblattfixatoren und der Beinmuskulatur, während der gesamten Studienzeit überprüfen.

Es wurden die maximale Anzahl der Sit-ups und der Liegestütze und die maximale erreichte Sprunghöhe beim „Jump and Reach“ erfasst (Bös et al., 1992).

Es handelt sich um eine ökonomische Testbatterie von Kraftübungen, die ohne großen organisatorischen Aufwand in den Räumen der Klinik stattfinden konnten. 
Eine Kraftmessung an speziellen Geräten war nicht möglich. Obwohl die Testbatterie Nachteile gegenüber Labortests hat, ist das Gütekriterium der Testökonomie jedoch ein wichtiges Argument. Durch den mehrmaligen Einsatz der gleichen Testbatterie sind jedoch gute Vergleiche hinsichtlich der motorischen Entwicklung möglich. Die Ergebnisse hinsichtlich der Sit-ups zeigen über die Baseline nur für die Kontrollgruppe einen leichten Anstieg der Leistungswerte. Da die Experimentalgruppe im gleichen Zeitraum keine Veränderung der Kraftleistung zeigt, lässt sich die Verbesserung in der Kontrollgruppe schwer erklären.

In der Interventionszeit kommt es durch beide Bewegungsprogramme zu einer signifikanten Verbesserung der Kraftausdauer der Bauchmuskulatur. Die Effektstärke war mit $\eta^{2}=0,558$ groß. In der Follow-up-Periode bleiben die Werte der beiden Gruppen stabil.

Obwohl die Aufgabe „Sit-ups“ für die Testpopulation modifiziert wurde, waren einige Probanden nicht in der Lage die Aufgabe durchzuführen. Gründe dafür waren körperliche Schmerzen und auch muskuläre Schwäche. Die Daten der Testaufgabe „Liegestütze“ zeigen, dass in der Baselinephase ein signifikanter Wertanstieg für beide Gruppen stattfindet. Auch diese Verbesserung ist schwer interpretierbar. Es wird angenommen, dass es beim zweiten Mal, dadurch dass die Übung bekannt war, zu ein einer größeren Einsatzbereitschaft und Motivation der Teilnehmer kam.

Nach der Behandlung steigt bei beiden Gruppen die Anzahl der Liegestützwiederholungen signifikant mit einer großen Effektstärke $\left(\eta^{2}=0,784\right)$.

Die Kraft der Arm- und Schultermuskulatur beider Gruppen ist am Ende des Treatments vergleichbar. Da jedoch am Anfang der Behandlung die Experimentalgruppe etwas zurücklag, ist die Verbesserung für diese Gruppe größer.

Während der Follow-up-Periode nimmt die Anzahl der geleisteten Liegestütze für beide Gruppen signifikant ab.

Es ist deutlich, dass sich ähnlich wie bei den „Sit-ups“ beide Programme hinsichtlich der Kraftkomponente als sehr wirksam erweisen. Diese Trainingseffekte waren zu erwarten, denn sowohl das psychoedukative Bewegungsprogramm als auch das Fitnesstraining beinhalteten gezielte Elemente des Muskelaufbautrainings nach funktionellen Gesichtspunkten. Erstaunlich ist, dass der Effekt der beiden Interventionen ähnlich ist, obwohl das Ausmaß an Kräftigungsübungen im Fitnessprogramm deutlich höher ist. 
Betrachtet man die Ergebnisse der Testaufgabe „Jump and Reach“ über die Gesamtstudienzeit, so zeigen sich keine wesentlichen Veränderungen hinsichtlich der Schnellkraft der Beinmuskulatur. Die Werte bleiben in beiden Gruppen über alle Messzeitpunkte in etwa gleich. Dieses Ergebnis erklärt sich zum einem dadurch, dass die Testmotivation der Teilnehmer bei der Aufgabe gering war. Obwohl auf die Beschaffenheit des Bodens und die Sicherheit bei der Landung geachtet wurde, ist das Springen für die Testpopulation mit einem erhöhten Sturzrisiko und somit mit einer gewissen Angst und Vorsicht verbunden. Die Testübung stellt eine beträchtliche biomechanische Beanspruchung dar (Bös et al., 1992), welche aufgrund des massiven Übergewichts noch intensiver wird.

Aus diesen Gründen wurde auf die Bewegungsform des Springens als Übungsform bei beiden Interventionen verzichtet. Ausgenommen sind die Trainingseinheiten im Wasser, in denen Sprünge in verschiedenen Ausführungen geübt wurden.

Zusammengefasst verdeutlichen die Daten, dass die bewegungstherapeutischen Maßnahmen einen günstigen Einfluss auf die Muskelkraft der Teilnehmer haben. Durch die bewegungstherapeutischen Programme konnten Veränderungen der Kraftfähigkeit bestimmter Muskelgruppen bewirkt werden. Es konnten keine Effektivitätsunterschiede hinsichtlich der Kraftfähigkeit zwischen beiden Gruppen festgestellt werden.

\subsection{Sport- und Bewegungsverhalten}

Inaktivität wird sowohl als eine Ursache als auch als Folge der Adipositas diskutiert. Das für die Erfassung der körperlichen Aktivität ausgewählte Instrument ist der Selbsteinschätzungsfragebogen. Um den mit derartigen Instrumenten verbundenen Effekt der sozialen Erwünschtheit zu kontrollieren, wurden entsprechende Kontrollfragen gestellt und differenzierte Angaben erbeten. So wurden die Aussagen der Teilnehmer weitmöglichst überprüft und relativiert.

Die Interpretation wird weiter durch das Phänomen der Überschätzung des Ausmaßes der körperlichen Aktivität erschwert. Wie in Kapitel 4.4.1 beschrieben, neigen Adipöse dazu, Dauer und Intensität ihrer Aktivität zu überschätzen. Es werden die Ergebnisse an den zwei Messzeitpunkten, Beginn und Follow-up der Untersuchung diskutiert. Die Frage „Treiben Sie Sport?“ führte zunächst zu einer dichotomen Teilung der Stichprobe in „Sportler“ oder „Nicht-Sportler“. Zu Beginn 
der Studie erklärten sich mehr als die Hälfte der Gesamtstichprobe, d.h. $60 \%$, zu „Nicht-Sportlern“.

Der Vergleich der Verteilung von „Sportlern“ und „Nicht-Sportlern“ auf die zwei Gruppen ist zu diesem Zeitpunkt statistisch nicht signifikant. Sechs Monate nach der Interventionszeit verringert sich der Anteil der „Nicht-Sportler“ mit $33 \%$ fast um die Hälfte. Dieser Anstieg der Sporttreibenden am Ende der Studie lässt sich zum Großteil durch den Anstieg der sportlich Aktiven in der Experimentalgruppe erklären. Während bei Studienbeginn nur 10 Teilnehmer der Experimentalgruppe aktiv sind, sind es zum Zeitpunkt der Katamnese 22. In der Kontrollgruppe kommen nur 3 Sportler dazu.

Des Weiteren wurde das Ausmaß der aktuellen sportlichen Aktivität anhand der vier Merkmale Intensität, Häufigkeit, Dauer und Art der sportlichen Aktivität erfasst. Hinsichtlich der Intensität, d.h. der subjektiv empfunden Intensität der Belastung im Training, zeigten sich Unterschiede zwischen den Gruppen. So gibt es nur in der Kontrollgruppe Probanden, die ihre Trainingsintensität als „locker und leicht“ empfinden. Die Mehrheit innerhalb beider Gruppen gab an, „flott und zügig“ zu trainieren. Es gibt nur einen kleinen Anteil von Probanden, die die Intensität ihres sportlichen Trainings als „hart und angestrengt“ bezeichnen. Dieser Anteil nimmt zum Messzeitpunkt der Katamnese $\mathrm{zu}$ und wird durch die Probanden der Experimentalgruppe bestimmt.

In Bezug auf die Häufigkeit (Trainingseinheiten pro Woche) gibt es unterschiedliche Angaben der aktiven Teilnehmer. Weniger als einmal die Woche trainierte nur ein Teilnehmer der Kontrollgruppe. Die meisten Teilnehmer beider Gruppen gaben an, einmal wöchentlich zu trainieren. Dieses bleibt bei beiden Messzeitpunkten trotz des leichten Anstiegs der „Sportler“ in der Katamnese konstant. Die Anzahl der Teilnehmer, die zweimal wöchentlich eine Trainingseinheit absolvierten, sind im Vergleich dazu wesentlich weniger. Während der Katamnese zeichnete sich allerdings ein Anstieg der Teilnehmer, die diese Frequenz angaben, ab. Ähnlich wenige Probanden trainierten zu Beginn der Studie dreimal oder öfter pro Woche aktiv. Auch dieses Verhältnis änderte sich nach der Interventionszeit, denn in der Katamnese berichteten mehr Probanden von einem dichteren Trainingswochenprogramm. Diese Zunahme betraf ausschließlich die Experimentalgruppe, in der Kontrollgruppe nahm die Anzahl der Probanden, die wöchentlich dreimal und öfter trainierten, sogar ab. 
Das Gesamtbild der Trainingsfrequenzen zeigte, dass mehr als die Hälfte der untersuchten aktiven Sportler einmal wöchentlich ihre Trainingseinheit absolviert. Diese Relation veränderte sich wenig nach der Behandlungsperiode. Die Ergebnisse der Katamnese zeigten, dass die dazu gewonnenen Sportler ein höher frequentiertes Trainingswochenprogramm auswählen. Besonders deutlich zeigte sich dieses in der Experimentalgruppe.

Die Antworten der Teilnehmer auf die Frage nach der Dauer (Stunden pro Woche) der sportlichen Aktivität können in zwei Kategorien eingeteilt werden. Die Dauer einer Trainingseinheit wurde entweder mit 20-40 min oder mehr als $40 \mathrm{~min}$ geschätzt. Vor der Untersuchung berichteten acht Probanden der Experimentalgruppe, dass ihre Trainingseinheit mehr als $40 \mathrm{~min}$ wöchentlich dauert, und nur zwei Probanden gaben eine Dauer von 20-40 min an. Bei den aktiven Sportlern der Kontrollgruppe ist die Verteilung auf die unterschiedliche Trainingsdauer mit sechs zu sieben Probanden ausgeglichen.

Im Zeitraum der Katamnese nahm die Trainingsdauer derer, die Sport treiben, zu, 18 \% der Experimentalgruppe trainierten mehr als $40 \mathrm{~min}$ und nur vier berichten von kurzen Trainingseinheiten mit einer Dauer von 20-40 min. In der Kontrollgruppe zeigten sich kaum Veränderungen bzgl. der Länge der Trainingseinheiten. Die Erfassung der sportlichen Aktivität durch die Selbstaussagen der Teilnehmer wurde durch die Frage nach den am häufigsten betriebenen Sportarten vervollständigt.

Es wurden insgesamt zwölf Sportarten genannt. Die populärsten waren Gymnastik, Schwimmen, Radfahren, Walking und Krafttraining. Es ist offensichtlich, dass diese Nennungen mit den allgemein empfohlenen Sportarten und Trainingsformen übereinstimmen.

Die Anzahl der Nennung von Gymnastik und Walking stieg nach der Intervention an, erklärbar dadurch, dass beide Trainingsformen Bestandteile der Behandlungsprogramme waren.

Fasst man alle diese Ergebnisse zusammen, zeigt sich eindeutig, dass nach der Behandlung insbesondere die Teilnehmer der Experimentalgruppe aktiver werden. Sie trainieren häufiger und länger als vor der Untersuchung. Dieses zeigt sich auch bei der Berechnung des Indexes zur sportlichen Aktivität. Mit Hilfe der Angaben zur Intensität, Häufigkeit und Dauer werden vier Typen hinsichtlich der sportlichen Aktivität gebildet (vgl. Kap. 6.11). Bezüglich der Kategorien „Regelmäßige Sportler“ und „Intensivsportler“ wird sechs Monate nach der Behandlung 
insbesondere in der Experimentalgruppe ein Zuwachs ersichtlich. Statistisch gesehen handelt es sich um signifikante Gruppenunterschiede. Die Anzahl der „,regelmäßigen Sportler“ in der Experimentalgruppe verdoppelt sich von sechs auf zwölf, während sie in der Kontrollgruppe nur mäßig von fünf auf sieben steigt. Die gesteigerte körperliche Aktivität in der Experimentalgruppe wird in der Kategorie „Intensivsportler“ noch deutlicher. In der Kontrollgruppe treibt zum Messzeitpunkt der Katamnese ein Teilnehmer intensiv Sport, während sich in der Experimentalgruppe die Zahl der „Intensivsportler“ von zwei vor der Untersuchung auf sieben nach der Behandlung erhöht.

Vergleicht man die sportlichen Aktivitäten der Teilnehmer mit den Richtlinien der “American College of Sports Medicine (ACSM)" (1995) und dem U.S. Department of Health and Human Services (1996), so findet man, dass selbst unter den „Intensivsportlern“ keiner die Empfehlungen bzgl. des Ausmaßes seiner sportlichen Aktivität erreicht.

Auf die oben genannte aktuelle Diskussion der sportlichen Aktivitäten folgt nun die Diskussion der „Sportgeschichte“ der Teilnehmer. Die Ermittlung dieser habituellen sportlichen Aktivität zu Beginn der Untersuchung gibt Aufschluss über die Sportbiographie der Teilnehmer und betont die Bedeutung von dauerhaften Verhaltensweisen für die Prognose des Gesundheitszustandes. Die Fragen wurden von beiden Gruppen ähnlich beantwortet (vgl. Kap. 6.11). Etwa die Hälfte der Gesamtstichprobe war vor 10 Jahren sportlich aktiv. Eine Teilnehmerin der Kontrollgruppe und drei der Experimentalgruppe berichteten, seit der Schulzeit ununterbrochen sportlich aktiv gewesen zu sein. Die meisten dieser Teilnehmer geben an, in der Vergangenheit im Vergleich zum aktuellen Messzeitpunkt mehr Sport getrieben zu haben. Auch hier konnte anhand des Fragebogens durch den Vergleich der gegen-wärtigen mit den vergangenen sportlichen Aktivitäten ein Index hinsichtlich der habituellen sportlichen Aktivität erstellt werden. Es wurden vier Kategorien gebildet. Anhand dieser Einteilung konnten $43 \%$ der Teilnehmer als „Nichtsportler“, $17 \%$ als „Abbrecher“, $12 \%$ als „Einsteiger“ sowie $28 \%$ als „,regelmäßig Sporttreibende“ charakterisiert werden. Der Prozentsatz der „regelmäßig Sporttreibenden“, d.h. Personen, die zur Zeit der Untersuchung wie auch vor 10 Jahren aktiv waren, ist sicherlich für die Stichprobe beachtlich groß. Nach der Einteilung der Probanden der beiden Gruppen in diese vier Kategorien ergeben sich keine Gruppenunterschiede. 
Die Beurteilung des Aktivitätsniveaus bei Adipösen ausschließlich über die Erfassung von sportlichen Aktivitäten greift zu kurz. Gerade um dem Konzept der „Lifestyle Activity“ gerecht $\mathrm{zu}$ werden, müssen die genannten körperlichen Aktivitäten, die nicht als sportliche Handlung gesehen werden, mit in die Diskussion einbezogen werden. Die Erfassung der körperlichen Aktivität in der Freizeit außer beim Sport ergibt sich aus der Tatsache, dass auch körperliche Alltagsaktivitäten gesundheitsfördernde Wirkungen haben können (vgl. Kap. 4.7). Auch wenn wie bei der sportlichen Aktivität keine Schätzung des Energieverbrauchs durch die körperliche Aktivität vorgenommen wurde, bekommt man ein umfassenderes Bild bzgl. des Bewegungsverhaltens der Teilnehmer.

Durch Selbstbeobachtung erfasst wurde zunächst die von den Teilnehmern an einem Werktag zurückgelegte Fußstrecke. Der Vergleich zwischen den Gruppen und den zwei Messzeitpunkten zeigt dabei keine signifikanten Unterschiede. Den vorliegenden Angaben zufolge legen die meisten Teilnehmer 1-2 km bzw. 3-5 km am Tag zurück. Die Prozentsätze der beiden Distanzkategorien verändern sich in der Katamnese nur für die Experimentalgruppe. Hier gab es eine Verschiebung von der 1-2 km Distanz zur 3-5 km Distanz. Bemerkenswert ist es, dass insgesamt sehr wenige Probanden die Kategorie „fast keine Fußstrecke“ und die Kategorie „6-9 km“ angeben. Es gab keinen Teilnehmer der „10 km und mehr“ täglich geht.

Ungefähr ein Drittel der Teilnehmer gaben am Anfang der Studie an, ihr Fahrrad in ihrem Alltag zu benutzen. Auch dieser Prozentsatz nahm in der Katamnese zu. Außerdem berichteten zu Studienbeginn sechzehn Teilnehmer der Gesamtstichprobe, fast täglich eine andere körperlich anstrengende Freizeittätigkeit auszuführen. Im Zeitraum der Nachuntersuchung wuchs die Anzahl dieser Teilnehmer auf zweiundzwanzig.

Obwohl die Erhebung zur Erfassung der körperlichen sportlichen Aktivität anhand des Fragebogens in der Form eines Interviews stattfand, kann „soziale Erwünschtheit" als ein die Antworten beeinflussendes Phänomen nicht ausgeschlossen werden. Die Tatsache, dass für die Untersuchung kein standardisiertes Instrument angewendet wurde, erschwert den Vergleich mit nationalen wie internationalen Daten.

Die Erfassung körperlicher Aktivität ist problematisch, und es besteht die Notwendigkeit, eine stärkere Standardisierung wie auch Kalibrierung der praktikablen Verfahren und Instrumente zu fördern. Weiterhin sollten Messmethoden entwickelt werden, die auch Lebensstil-Aktivitäten (z.B. in Beruf, Haushalt, Erziehung und 
Pflege) erfassen, die den Lebensbereichen und Aktivitätsmustern von Menschen mit Adipositas angemessener sind. Moderne Informationstechnologien könnten für die Neuentwicklung von Verfahren stärker als bisher genutzt werden.

Zusammenfassend weisen die Ergebnisse der Untersuchung hinsichtlich der sportlich-körperlichen Aktivität auf eine heterogene Untersuchungsgruppe hin. Es handelt sich um eine Stichprobe, in der der Anteil der Aktiven beachtlich ist. Obwohl es statistisch nicht eindeutig belegt wurde, scheint das psychoedukative Bewegungsprogramm im Vergleich zur Kontrollgruppe einen größeren Einfluss auf das Sportund Bewegungsverhalten der Teilnehmer zu haben. 


\section{Zusammenfassung und Ausblick}

Bewegungstherapeutische Maßnahmen gelten bei der Behandlung der Adipositas als unerlässlich und haben sich im klinischen wie im ambulanten Bereich im Rahmen multimodaler Therapiekonzepte als feste Bestandteile durchgesetzt. Unterstützt durch die Grundannahme, dass die Ursache der Adipositas an der Disbalance von Energieaufnahme und Energieabgabe liegen, werden bewegungstherapeutische Konzepte durch eine biophysiologische Sichtweise beeinflusst. Die wichtige Rolle der Bewegung im Sinne von körperlich-sportlicher Aktivität für die Entstehung sowie für die Behandlung der Adipositas ist ausreichend fundiert. Mehrere Untersuchungen der letzten Jahre haben die Wirksamkeit sport- und bewegungstherapeutischer Behandlungsprogramme bei Adipositas nachweisen können (Fogelholm, Stallknecht \& Van Baak, 2006; Bensimhon, Kraus \& Donahue, 2006). Diese Programme basieren in erster Linie auf intensiven sportlich - aktivierenden Maßnahmen mit der Gewichtsreduktion als Hauptzielsetzung. Es ist bekannt, wie schwierig es ist, eine langfristige Gewichtsstabilisierung nach einer Gewichtsreduktion zu erreichen. Auch die langfristige Aufrechterhaltung der körperlichen sportlichen Aktivität stellt für Menschen mit Adipositas eine Herausforderung dar. Aus sportwissenschaftlicher Sicht liegt die höchste Priorität darin, die vorhandenen sporttherapeutischen Behandlungsansätze effizienter zu gestalten.

Die Tatsache, dass Adipositas nicht nur somatische Folgeerscheinungen hat, sondern auch psychosoziale Auswirkungen, z.B. auf die Lebensqualität, sowie die Tatsache, dass eine langfristige Aufrechterhaltung der körperlich-sportlichen Aktivitäten von Menschen mit Adipositas ungenügend realisiert wird, sollten dazu führen, dass weitere bewegungstherapeutische Interventionen entwickelt und evaluiert werden. Abgesehen von Untersuchungen zum Thema Trainingsmaßnahmen und Gewichtsmanagement, die vorwiegend aus dem angloamerikanischen und dem skandinavischen Raum stammen, gibt es kaum sportwissenschaftliche Studien, die die Wirksamkeit bewegungstherapeutischer Interventionen für adipositasrelevante Themen erfassen. Es fehlen wissenschaftlich überprüfte Aussagen aus den Bereichen der allgemeinen körperlichen Funktionsfähigkeit, des Körperbilds, des Essverhaltens und der Sportmotivation. Zusammenhänge zwischen körperlicher Aktivität, Körperkonzept und Selbstwerteinschätzung sowie kognitiver Entwicklung und sozialer 
Kompetenzentwicklung weisen darauf hin, dass Bewegungstherapie durch psychotherapeutische Inhalte effektiver werden könnte.

Der im Theorieteil beschriebene „State of the Art“ zur wissenschaftlichen Überprüfung der Wirksamkeit sporttherapeutischer Maßnahmen hinsichtlich therapeutisch relevanter Aspekte wie Lebensqualität und Körperbild zeigte, dass Behandlungsprogramme, die Sporttherapie mit Psychoedukation und Körpertherapie kombinieren, nicht ausreichend erforscht sind. Die vorliegende Studie ist folglich als ein Schritt anzusehen, die aktuellen Themen der Adipositasforschung aufzunehmen und die Entwicklung wirkungsvoller Therapieansätze zu unterstützen.

Nach der theoretischen Einordnung des psychoedukativen Bewegungsprogramms in bestehende sporttherapeutische wie verhaltensorientierte Behandlungsformen wurden in der empirischen Untersuchung die Effekte des theoretisch begründeten Behandlungsansatzes über die praktische Anwendung evaluiert.

In dieser randomisierten, kontrollierten Studie wurde überprüft, ob für die Zielgruppe der Menschen mit Adipositas das psychoedukative Bewegungsprogramm konventionellen Fitnessprogrammen überlegen ist.

Für die Untersuchung wurde eine anfallende Stichprobe bestehend aus 58 Menschen mit Adipositas Grad II akquiriert und in Kontroll- und Experimentalgruppe unterteilt. 30 Teilnehmer wurden mit dem psychoedukativ - orientierten Bewegungsprogramm in Form eines standardisierten Programms behandelt („Psychoedukatives Bewegungsprogramm für Menschen mit Adipositas"). Die übrigen 28 Teilnehmer der Kontrollgruppe nahmen über den gleichen Behandlungszeitraum an einem standardisierten Fitnesstraining teil. Alle Teilnehmer wurden systematisch 4 Wochen vor Programmbeginn, bei Programmbeginn, 8 Wochen nach Programmbeginn (Ende der Intervention) und 6 Monate nach Ende des Programms untersucht. Die Vergleichbarkeit der beiden Gruppen bei Aufnahme in die Studie war gewährleitstet. Zur Interpretation der Studienergebnisse und, um die Frage nach einer Generalisierbarkeit der Ergebnisse zu beantworten, müssen einige Besonderheiten berücksichtigt werden. Es handelt sich um eine Stichprobe von Probanden mit einer Adipositas zweiten Grades (Mittelwert BMI = 38,8). Die Teilnehmer an der vorliegenden Studie waren hoch motiviert, ein Umstand, der sich u. a. in der geringen Anzahl von drei Studienabbrechern widerspiegelt.

Weiterhin sollte berücksichtigt werden, dass die Mehrheit der Teilnehmer, 53 von insgesamt 58, Frauen waren. Dies ist allerdings in der Adipositasforschung keine 
Seltenheit, denn der Leidensdruck bei Übergewicht und der Wunsch nach einer Gewichtsabnahme sind bei Frauen größer (Friedman \& Brownell, 2002).

Aus der Untersuchung können Schlussfolgerungen auf verschiedenen Ebenen abgeleitet werden. An unterschiedlichen körperlichen wie psychischen Messfaktoren wurde offensichtlich, dass es sich bei den Übergewichtigen und Menschen mit Adipositas um eine sehr heterogene Gruppe handelt.

Das initiale Niveau der körperlichen Fitness war sehr niedrig. Trotz der signifikanten Verbesserungen in allen sportmotorischen Bereichen durch das Training lagen die Werte der Stichprobe auch bei Behandlungsende deutlich unter der Norm. In der Praxis zeigte sich, dass aufgrund des Übergewichts und den damit verbundenen Gesundheitsrisiken, Begleiterkrankungen sowie motorischen Einschränkungen gängige sportmotorische Testverfahren ungeeignet und zum Teil sogar gefährlich sind. Sie mussten für den Zweck der Untersuchung modifiziert werden. Spezifische, standardisierte sportmotorische Instrumente für die Population der übergewichtigen Bevölkerung fehlen und sollten entwickelt werden.

Die Untersuchung bestätigt die therapeutische Wirkung beider Bewegungsprogramme auf einer grundlagenorientierten sport- bzw. gesundheitswissenschaftlichen Ebene. Es können zwei übergeordnete Aussagen gemacht werden:

I. Sowohl in der Experimentalgruppe als auch in der Kontrollgruppe haben sich der körperliche Funktions- und Leistungsstatus und einige psychologische Aspekte signifikant gebessert.

II. Betrachtet man die vergleichende statistische Auswertung zwischen Experimental- und Kontrollgruppe, so ergeben sich Hinweise für eine bessere Wirksamkeit des psychoedukativen Bewegungsprogramms.

Die Aussage I wird dadurch gestützt, dass beide Bewegungsprogramme zu einem mäßigen Gewichts- bzw. Körperfettverlust führen. Beide bewegungstherapeutischen Programme haben einen Einfluss auf die subjektive Lebensqualität. Nach der Interventionsperiode konnte für beide Gruppen ein signifikanter Zuwachs an Lebensqualität in fast allen Subskalen (sieben von acht) des SF-36 erreicht werden. Das Ausmaß der Verbesserung war so deutlich, dass die körperliche Lebensqualität der Probanden zum Ende der Behandlung nur geringfügig unter der der Normalbevölkerung und oberhalb der von Hypertonie- bzw. Diabetespatienten lag. 
Die Bewegungsprogramme verbesserten das Gesamtkörperkonzept signifikant. Nach der Behandlung zeigen sich in sieben der neun Subskalen des Fragebogens zum Körperkonzepts (FKKS) signifikante Verbesserungen. Durch die Bewegungsprogramme verschwand die depressive Symptomatik, die sowohl in der Versuchsgruppe als auch in der Kontrollgruppe gemessen wurde. Beide bewegungstherapeutischen Programme haben deutliche Effekte auf die Fitness. Dies wurde durch die Erfassung der aeroben Ausdauerleistung sowie der Kraft- und Koordinationsfähigkeit belegt. Die Akzeptanz beider bewegungstherapeutischer Programme durch die Teilnehmer war sehr hoch.

Eine Überlegenheit des psychoedukativen Bewegungsprogramms zeigte sich hinsichtlich des Körpergewichts in der Follow-up-Periode. Die Experimentalgruppe konnte die Gewichtsreduktion besser halten. Weiterhin führte das psychoedukative Bewegungsprogramm im Vergleich zum Fitnesstraining zu einem signifikant stärkeren Zuwachs der psychischen Lebensqualität. Auch dieser Gruppenunterschied blieb im Zeitraum der Katamnese erhalten. Eine nachhaltige Wirkung des psychoedukativen Bewegungsprogramms ließ sich vor allem für das Körperkonzept belegen, und auch die Stimmung wurde durch das psychoedukative Bewegungsprogramm wirkungsvoller aufgehellt. Die Abnahme der Depressivität war in der Experimentalgruppe signifikant größer und blieb auch in der Katamnese bei mehr Patienten unauffällig. Es zeigte sich, dass das psychoedukative Bewegungsprogramm im Gegensatz zum Fitnesstraining einen Einfluss auf die essstörungsspezifische Pathologie, konkret auf die Binge Eating Disorder, der Teilnehmerinnen hatte. Hinsichtlich der Wirkungsweise der Bewegungstherapie wurde das psychoedukative Bewegungsprogramm als wirkungsvoller in Bezug auf Bewegungserleben und Wohlsein eingeschätzt als das Fitnesstrainingsprogramm. Die Analyse der Daten zum Bewegungsverhalten zum Messzeitpunkt der Katamnese zeigte eine deutlichere, wenn auch rein deskriptive und statistisch nicht signifikante Zunahme der körperlichen und sportlichen Aktivität der Experimentalgruppe im Vergleich mit der Kontrollgruppe. Die Experimentalgruppe unterscheidet sich somit bzgl. der Mehrzahl der gemessenen Parameter signifikant von der Kontrollgruppe. Die deutlichsten Effekte des psychoedukativen Bewegungsprogramms zeigen sich hinsichtlich der verbesserten psychischen und nicht der sportspezifischen Parameter. 
Die Hypothese einer größeren Effizienz in der Experimentalgruppe findet in der durchgeführten Untersuchung eine Unterstützung.

Abschließend kann festgestellt werden, dass das psychoedukative Bewegungsprogramm eine effektive Therapieform darstellt, welche nicht nur physiologische, sondern insbesondere psychologische Aspekte der Adipositasbehandlung beeinflussen kann. Die vorliegende Arbeit belegt, dass eine Verbindung von bewegungstherapeutischen Behandlungsmethoden und Verhaltenstherapie die Qualität einer Behandlung verbessert. Die Sportwissenschaft, die den Lerntheorien ohnehin eng verbunden ist, sollte den Bereich der Verhaltenstherapie durch Sport und Bewegung verstärkt in die Lehrpläne der Sporttherapeuten einbeziehen. 



\section{Literaturverzeichnis}

Alexandridis, K. (2005). Bewegungstherapie und Bulimia nervosa Evaluation einer stationären Körpertherapie. dissertation.de - Verlag im Internet GmbH.

American Psychiatric Association [APA], (1994). Diagnostic and statistical manual of mental disorders (4th ed.). Washington DC: Author.

Andersen, R.E., Wadden, T.A., Bartlett, S.J., Zemel, B., Verde, T.J. \& Franckowiak, S.C. (1999). Effects of lifestyle activity vs. structured aerobic exercise in obese women: a randomized trial. Journal of the American Medical Association, 281, 335340 .

Andersen, R.E. (Ed.). (2003). Obesity Etiology Assessment Treatment and Prevention. Champaign, IL: Human Kinetics.

Aronne, L.J. (2002). Current pharmacological treatments for obesity. In C.G. Fairburn \& K.D. Brownell (Ed.), Eating Disorders and Obesity A Comprehensive Handbook (Second Edition) (pp. 551-556). New York: Guilford Press.

Atkinson, R.L. (1999). Etiologies of obesity. In D.J. Goldstein (Ed.), The Management of eating disorders and obesity (pp. 84-92). Totowa, New Jersey: Humana Press.

Bässler, R. (1988). Methodische Überlegungen zur empirischen Erhebung und Beschreibung der „Teilnahme der Bevölkerung am Sport“. SWS- Rundschau, 28, 191-197.

Baker, C.W. \& Brownell, K.D. (2000). Physical activity and maintenance of weight loss: physiological and psychological Mechanisms. In C. Bouchard (Ed.), Physical Activity and Obesity (pp. 311-328). Champaign, IL: Human Kinetics.

Ballor, D.L., \& Poehleman, E.T. (1994). Exercise-training enhances fat-free mass preservation during diet-induced weight loss: a meta-analytical finding. International Journal of Obesity, 18, 35-40.

Bandura, A. (1995). Exercise of personal and collective efficacy in changing societies. In A. Bandura, (Ed.), Self-efficacy in changing societies (pp. 1-45). New York: Cambridge University Press.

Benecke, A. \& Vogel, H. (2003). Übergewicht und Adipositas Gesundheitsberichterstattung des Bundes -Heft 16. Zugriff am 10 Februar 2005 unter

http://www.rki.de/cln_006/nn_226040/DE/Home/homepage_node.html_nnn=true

Benecke, A. (2002). Verhaltenstherapie bei Adipositas. Verhaltenstherapie, 12 (4), 297-309. 
Bergmann, K.E., Bergmann, R.E. \& Richter, R. (2005). Epidemiologie von Erwachsenen in Europa. In H. Erbersdolber, H. Heseker \& Wolfram G. (Hrsg.), Adipositas - Eine Herausforderung für's Leben? (S. 13-29). Stuttgart: Hoffmann Schorndorf.

Bensimhon, D.R., Kraus, W.E. \& Donahue, M.P. (2006). Obesity and physical activity: A review. American Heart Journal, 151, 598-603.

Biddle, S. \& Mutrie, N. (2001). Psychology of Physical Activity. London: Routledge.

Bielefeld, J. (Hrsg.). (1991). Körpererfahrung. Grundlagen menschlichen Bewegungsverhaltens (2., Aufl.). Göttingen: Hogrefe.

Biospace, (2001). Lookin `Body InBody Data Management System User Guide. Seoul: Biospace Corporation Limited.

Björntorp, P. (2002). Definition and classification of obesity. In C.G. Fairburn \& K.D. Brownell (Ed.), Eating Disorders and Obesity A Comprehensive Handbook (Second Edition) (pp. 337-381). New York: Guilford Press.

Black, A.E., Coward, W.A., Cole, T.J. \& Prentice, A.M. (1996). Human Energy expenditure in affluent societies: An analysis of 574 doubly-labeled water measurements. European Journal of Clinical Nutrition, 50, 72-92.

Blair, S.N. \& Brodney, S. (1999). Effects of physical inactivity and obesity on morbidity and mortality: Current evidence and research issues. Medicine and Science in Sports and Exercise, 31, (11, Suppl.) 646-662.

Blair, S.N. \& Holder, S. (2002). Exercise in the management of obesity. In C.G. Fairburn \& K.D. Brownell (Ed.), Eating Disorders and Obesity A Comprehensive Handbook (Second Edition) (pp. 518-523). New York: Guilford Press.

Blair, S.N. \& Leermakers, E.A. (2004). Exercise and weight management. In T.A. Wadden \& A.J. Stunkard (Ed.), Handbook of obesity Treatment (pp. 283-300). New York: Guilford Press.

Bortz, J. (1985). Lehrbuch der Statistik für Sozialwissenschaftler (2. vollständig neu bearbeitete und erweiterte Aufl.). Berlin: Springer.

Bortz, J., Lienert, G. A. \& Boehnke, K. (1990). Verteilungsfreie Methoden in der Biostatistik. Heidelberg: Springer.

Bös, K., Wydra, G. \& Karisch, G. (1992). Gesundheitsförderung durch Bewegung, Spiel und Sport. Erlangen: perimed

Bös, K., (1994). Handbuch für Walking. Aachen: Meyer \& Meyer.

Bosy-Westphal, A., Kromeyer-Hausschild, K., Pirlich, M., Schlattmann A., Scholz, G. H., Müller, M.J., (2006). Körperzusammensetzung - Was kann man wie und mit welchem Wert in der Praxis messen?. Aktuelle Ernährungsmedizin, 31, 189-195. 
Bouchard, C. (Ed.). (2000). Physical Activity and Obesity. Champaign, IL: Human Kinetics.

Bouchard, C., (2000). Introduction. In C. Bouchard (Ed.), Physical Activity and Obesity (pp. 3-19). Champaign, IL: Human Kinetics.

Bray, G.A. (2000). Overweight, mortality, and morbidity. In C. Bouchard (Ed.), Physical Activity and Obesity (pp. 31-53). Champaign, IL: Human Kinetics.

Brownell, K.D., Stunkard, A.J. \& Albaum, J.M. (1980). Evaluation and modification of exercise patterns in the natural environment. American Journal of Psychiatry 137, $1540-1545$.

Brownell, K.D. (1995). Exercise in the treatment of obesity. In C.G. Fairburn \& K.D. Brownell (Ed.), Eating Disorders and Obesity A Comprehensive Handbook (pp. 473478). New York: Guilford Press.

Brownell, K.D. (2002). The environment and obesity. In C.G. Fairburn \& K.D. Brownell (Ed.), Eating Disorders and Obesity A Comprehensive Handbook (Second Edition) (pp. 433-438). New York: Guilford Press.

Bühl, A. \& Zöfel, P. (2002). SPSS 11. Einführung in die moderne Datenanalyse unter Windows (8. Auflage). München: Pearson Studium.

Bullinger, M. \& Kirchberger, I. (1998). Der SF-36 Fragebogen zum Gesundheitszustand: Handbuch für die deutschsprachige Fragebogenversion. Göttingen: Hogrefe.

Bundesagentur für Außenwirtschaft (2005).US-Sportmarkt boomt wieder. Zugriff am 20. April 2006 unter http://www.bfai.de/ext/ExportEinzelsicht/DE/Content/_SharedDocs/Links-EinzeldokumenteDatenbanken/fachdokument,templateId=renderPrint/MKT20050301090813.pdf

Cohen, J. (1988). Statistical power analysis for the behavioral sciences ( $2^{\text {nd }}$ edition). Hillsdale, New Jork: Erlbaum.

Cash, T.F. (1990). The psychology of physical appearance: Aesthetics, attributes, and images. In T.F. Cash \& Pruzinsky (Ed.), Body images: Development, deviance, and change (pp. 51-79). New York: Guilford Press.

Calle, E.E., Thun, M.J., Petrelli, J.M., Rodriguez, C. \& Heath C.W. (1999). Body Mass Index and mortality in a prospective cohort of U.S. adults. The New England Journal of Medicine, 341, 1097-1105.

Caspersen, C.J., Powell, K.E. \& Christensen, G.M. (1985). Physical activity, exercise, and physical fitness: definitions and distinctions for health-related research. Public Health Reports, 100, 126-131. 
Catanese, D.M., Hyder, M.L., Poston, W.S.C. \& Foreyt, J.P. (2003). Future directions in treating obesity. In R.E. Andersen (Ed.), Obesity Etiology Assessment Treatment and Prevention (pp. 271-289). Champaign, IL: Human Kinetics.

Cooper, Z. \& Fairburn, C.G. (2004). Cognitive- behavioral treatment of obesity. In T.A. Wadden \& A.J. Stunkard (Ed.), Handbook of obesity Treatment (pp. 465-479). New York: Guilford Press.

Curioni, C.C. \& Lourenco, P.M. (2005). Long-term weight loss after diet and exercise: a systematic review. International Journal of Obesity, 29, 1169-1174.

Deimel, H. (2001). Möglichkeiten und Grenzen einer integrativ ausgerichteten Bewegungstherapie im ambulanten Bereich - Erste Ergebnisse. In Fachverband Sucht e.V. (Hrsg.), Rehabilitation der Suchterkrankungen - mehr als Psychotherapie (S. 148-155). Geesthacht: Neuland Verlag.

Deusinger, I. M. (1986). Die Frankfurter Selbstkonzeptskalen - FSKS. Ein Verfahren zur Messung von 10 differenzierten Selbstkonzepten. Göttingen: Hogrefe.

Deusinger, I. M. (1998). Die Frankfurter Selbstkonzeptskalen (FKKS). Testmanual. Göttingen: Hogrefe.

Deutsche Adipositas Gesellschaft (2006). Evidenzbasierte Leitlinie Prävention und Therapie der Adipositas Version 2006. Zugriff am 20. März 2006 unter http://www.adipositas-gesellschaft.de/daten/Adipositas-Leitlinie-2006.pdf

Deutsche Adipositas Gesellschaft (2006). Evidenzbasierte Leitlinie Chirurgische Therapie der extremen Adipositas. Zugriff am 20. März 2006 unter http://www.adipositas-gesellschaft.de/daten/Leitlinie-Chirurgie.pdf

de Zwaan, M. (2002). Binge eating disorder (BED) und Adipositas. Verhaltenstherapie, 12 (4), 288-295.

DiPietro, L., Kohl, H.W., Barlow, C.E. \& Blair S.N. (1998). Improvements in cardiorespiratory fitness attenuate age-related weight gain in healthy men and women: the Aerobics Center Longitudinal Study. International Journal of Obesity, $22,55-62$.

Dunn, A.L., Andersen, R.E. \& Jakicic, J.M. (1998). Lifestyle physical activity interventions. History, short- and long- term effects, and recommendations. American Journal of Preventive Medicine, 15 (4), 398-412.

Dunn, A.L., Marcus, B.H., Kampert, J.B., Garcia, M.E., Kohl, H.W. \& Blair, S.N. (1999). Comparison of lifestyle and structured interventions to increase physical activity and cardiorespiratory fitness: A randomized trial. Journal of the American Medical Association, 281, 327-334.

Duperly, J. (1988).Über den Einfluss der Bewegungstherapie auf Insulin und Leptin bei Übergewicht. Unveröff. Dissertation, Deutsche Sporthochschule, Köln. 
Ehrhardt, M. \& Seeck, U. (1990). Essstörungen. In Bundesministerium für Arbeit und Sozialordnung (Hrsg.), Bewegung, Spiel und Sport mit Behinderten und von Behinderung Bedrohten. Indikationskatalog und Methodenmanual, Bd. 3. Indikationskatalog: Sinnesbehinderungen. Lern- und geistige Behinderungen. Psychische Störungen (S. 1119-1155). Bonn.

Ehrsam, R., Stoffel, S., Mensink, G. \& Melges, T. (2004).Übergewicht und Adipositas in den USA, Deutschland, Österreich und der Schweiz. Deutsche Zeitschrift für Sportmedizin, 55 (11), 278-285.

Ellert, U. \& Bellach, B-M. (1999). Der SF-36 im Bundes-Gesundheitssurvey Beschreibung einer aktuellen Normstichprobe. Gesundheitswesen, 61 (Sonderheft 2), 184-190.

Ellrott, T. \& Pudel, V. (1998). Adipositastherapie (2., aktualisierte Aufl.). Stuttgart: Thieme.

Ellrott, T. (2003). Medizinische Behandlung, Medikamente und chirurgische Maßnahmen. In F. Petermann \& V. Pudel (Hrsg.), Übergewicht und Adipositas (S. 183-206). Göttingen: Hogrefe.

Europäisches Informationszentrum für Lebensmittel. (2006). Sport und körperliche $\begin{array}{lllll}\text { Bewegung Zugriff am } 3 . \quad \text { März } 2006 & \text { unter }\end{array}$ http://www.eufic.org/jarticle/de/lebensmittel-ernahrungsfuhre/sport-korperlichebewegung/expid/basics-korperlichen-aktivitaten/

Fairburn, C.G. \& Brownell, K.D. (Ed.). (2002). Eating Disorders and Obesity A Comprehensive Handbook (Second Edition). New York: Guilford Press.

Falkner, N.H., French, S.A., Jeffery, R.W., Neumark-Sztainer, D. Sherwood, N.E. \& Morton, N. (1999). Mistreatment due to weight: Prevalence and sources of perceived mistreatment in women and men. Obesity research, 7, 572-576.

Fichter, M.M. \& Quadflieg, N. (1999). Strukturiertes Inventar für Anorektische und Bulimische Essstörungen (SIAB). Göttingen: Hogrefe.

Field, A.E., Barnoya, J. \& Colditz, G.A. (2004). Epidemiology and health and economic consequences of obesity. In T.A. Wadden \& A.J. Stunkard (Ed.), Handbook of obesity Treatment (pp. 3-18). New York: Guilford Press.

Fitzgibbon, M.L.,Stolley, M.R. \& Kirschenbaum, D.S. (1993). Obese people who seek Treatment have different characteristics than those who do not seek treatment. Health Psychology, 12, 342-345.

Flegal, K.M., Graubard, B.I., Williamson, D.F. \& Gail, M.H. (2005). Excess deaths associated with underweight, overweight, and obesity. JAMA, 293, 1861-1867.

Fogelholm, M. \& Kukkonen-Harjula, K. (2000). Does physical activity prevent weight gain-a systematic review. Obesity Reviews,1 (2), 95-111. 
Fogelholm, M., Stallknecht, B. \& Van Baak, M. (2006). Exercise and Obesity. European Journal of Sport Science, 6 (1), 15-24.

Fontaine, K.R. \& Bartlett, S.J. (2003). Health-Related Quality of Life in Obese Individuals . In R.E. Andersen (Ed.), Obesity Etiology Assessment Treatment and Prevention (pp. 59-72). Champaign, IL: Human Kinetics.

Foreyt, J.P., Brunner, R.L., Goodrick, G.K., St Jeor, S.S.T. \& Miller, G.D. (1995). Psychological correlates of reported physical activity in normal-weight and obese adults: the Reno diet-heart study. International Journal of Obesity and Related Metabolic Disorder, 19 (4 Suppl.), 69-72.

Foreyt, J.P. \& Poston, W.S., (1998). What is the role of cognitive- behaviour therapy in patient management? Obesity Research, 6 (Suppl.1), 18-22.

Foster, G.D. \& Wadden, T.A. (2002). Social and psychological effects of weight loss. In C.G. Fairburn \& K.D. Brownell (Ed.), Eating Disorders and Obesity A Comprehensive Handbook (Second Edition) (pp. 500-504). New York: Guilford Press.

Fox, K.R. (1992). A clinical approach to exercise in the severely obese. In T.A. Wadden \& T.B. Van Itallie (Ed.), Treatment of severe obesity by diet and lifestyle modification (pp. 354-382). New York: Guilford Press.

Franckowiak, S.C. \& Andersen, R.E. (2003). Physical activity treatment. In R.E. Andersen (Ed.), Obesity Etiology Assessment Treatment and Prevention (pp. 187201). Champaign, IL: Human Kinetics.

French, S.A., Jeffery, R.W., Forster, J.L., McGovern, P.G., Kelder, S.H. \& Baxter, J.E. (1994). Predictors of weight change over two years among a population of working adults: the Healthy Worker Project. International Journal of Obesity, 18, 145-154.

Friedman, M.A. \& Brownell, K.D. (2002). Psychological consequences of obesity. In C.G. Fairburn \& K.D. Brownell (Ed.), Eating Disorders and Obesity A Comprehensive Handbook (Second Edition) (pp. 393-398). New York: Guilford Press.

Fuchs, R. \& Schwarzer, R. (1994). Selbstwirksamkeit zur sportlichen Aktivität: Reliabilität und Validität eines neuen Messinstruments. Zeitschrift für Differentielle und Diagnostische Psychologie, 15, 141-154.

Fuchs, R. (2003). Sport, Gesundheit und Public Health. Göttingen: Hogrefe.

Garner, D.M. (1997). The 1997 body image survey results. Psychology Today, p. 30.

Gortmaker, S.L., Must, A., Perrin, J.M., Sobol, A.M. \& Dietz, W.H. (1993). Social and economic consequences of overweight in adolescence and young adulthood. New England Journal of Medicine, 329, 1008- 1012. 
Garrow, J.S. \& Summerbell, C.D. (1995). Metaanalysis: effect of exercise, with or without dieting, on the body composition of overweight subjects. European Journal Clinical Nutrition, 49, 1-10.

Graf, C. \& Predel, H.G. (2005). Die Rolle der körperlichen Aktivität in der Adipositasentstehung. In H. Erbersdolber, H. Heseker \& Wolfram G. (Hrsg.), Adipositas - Eine Herausforderung für's Leben? (S. 55-62). Stuttgart: Hoffmann Schorndorf.

Grillo, J.M., Brownell, K.D. \& Stunkard, A.J. (1993). The metabolic and psychological importance of exercise in weight control. In A.J. Stunkard \& T.A. Wadden (Ed.), Obesity: theory and therapy (Second Edition) (pp. 253- 273). New York: Raven Press.

Grillo, J.M. (2003). Treatment of obesity: an integrative model. In J.K. Thompson (Ed.), Body Image, Eating Disorders, and Obesity (pp. 389-423). Washington, DC: American Psychological Association.

Grundy, S.M., Blackburn, G., Higgins, M., Lauer, R., Perri, M.G. \& Ryan, D. (1999). Physical activity in the prevention and treatment of obesity and its comorbidities. Medicine \& Science in Sports \& Exercise, 31 (11 Suppl.), 502-508.

Grundy, S.M., Cleemann, J.I., Daniels, S.R., Donato, K.A., Eckel, R.H., Franklin, B.A., Gordon, D.J., Krauss, R.M., Savage, P.J., Smith, S.C. Jr, Spertus, J.A. \& Costa F. (2005). Diagnosis and management of the Metabolic Syndrome. An American Heart Association/National Heart, Lung, and Blood Institute scientific statement. Circulation, 112, 2735-2752.

Hauner, H. \& Berg, A. (2000). Körperliche Bewegung zur Prävention und Behandlung der Adipositas. Deutsches Ärzteblatt, 97 (12), 768-774.

Hautzinger, M., Bailer, M., Worall, H. \& Keller, F. (1995). Beck Depressions Inventar (BDI) (2., überarb. Aufl.). Bern: Huber.

Hebebrand, J., Dabrock, P., Lingenfelder, M., Mand, M., Rief, W. \& Voit, W. (2004). Ist Adipositas eine Krankheit? Interdisziplinäre Perspektiven. Deutsches Ärzteblatt, 101 (37), 2468-2476.

Herpertz, S. \& Saller, B. (2001). Psychosomatische Aspekte der Adipositas. Psychotherapie, Psychosomatik, medizinische Psychologie, 51, 336-349.

Herpertz, S. \& Senf, W. (2003). Psychotherapie der Adipositas. Deutsches Ärzteblatt, 100 (20), 1367-1373.

Hill, J.O. (2002). An overview of the Etiology of Obesity. In C.G. Fairburn \& K.D. Brownell (Ed.), Eating Disorders and Obesity A Comprehensive Handbook (Second Edition) (pp. 460-464). New York: Guilford Press. 
Hölter, G. (2004). Diagnostik des körper- und bewegungsbezogenen Erlebens und Verhaltens. In K. Schüle \& G. Huber (Hrsg.), Grundlagen der Sporttherapie (2. Auflage) (S. 109-120). München: Elsevier.

Horgen, K.B. \& Brownell, K.D. (2004). Confronting the toxic environment: Environmental and public Health actions in a world crisis. In T.A. Wadden \& A.J. Stunkard (Ed.), Handbook of obesity Treatment (pp. 95-106). New York: Guilford Press.

Huber, G. (2004). Abnormales Gewicht als normale Antwort auf eine abnormale Umwelt. Bewegungstherapie und Gesundheitssport, 20 (6), 223-227.

Huber, G. (2007). Fitnesslügen. Bewegungstherapie und Gesundheitssport, 23 (1), 32.

Hübschmann, C. (1997). Effektivitätsüberprüfung einer ambulanten Sporttherapie im Rahmen eines multimodalen Rehabilitationskonzeptes für Personen mit Adipositas der BKK Bayer. Unveröff. Dipl. Arbeit, Deutsche Sporthochschule, Köln.

Husemann, B. (1997). Die chirurgische Therapie der extremen Adipositas. Deutsches Ärzteblatt, 94 (33), 2132-2136.

Husemann, B. (1998). Chirurgische Therapie der extremen Adipositas. In J.G. Wechsler (Hrsg.), Adipositas Ursachen und Therapie (S. 267-289). Berlin: Blackwell.

Jakicic, J.M., Wing, R.R., Butler, B.A. \& Robertson, R.J. (1995). Prescribing exercise in multiple short bouts versus one continuous bout: effects on adherence, cardiorespiratory fitness, and weight loss in overweight women. International Journal of Obesity, 19, 893-901.

Jakicic, J.M., Wing, R.R., Butler, B.A. \& Jeffery, R.W. (1997). The relationship between the presence of exercise equipment in the home and physical activity level. American Journal of Health Promotion, 11 (5), 363-365.

Jakicic, J.M., Winters, C., Lang, W. \& Wing, R.R. (1999). Effects of intermittent exercise and use of home exercise equipment on adherence, weight loss, and fitness in overweight women. Journal of the American Medical Association, 282, 15541560 .

Jakicic, J.M. (2003). Physical activity as a therapeutic modality. In R.E. Andersen (Ed.), Obesity Etiology Assessment Treatment and Prevention (pp. 203-215). Champaign, IL: Human Kinetics.

Jakicic, J.M. \& Otto, A.D. (2005). Physical activity considerations for the treatment and prevention of obesity. The American Journal of clinical Nutrition, 82 (suppl.), 226-229. 
Johnson, C. (2004). Obesity, weight management and self-esteem. Obesity and body image disturbance. In T.A. Wadden \& A.J. Stunkard (Ed.), Handbook of obesity Treatment (pp. 480-493). New York: Guilford Press.

Kanfer, F.H., Reinecker, H. \& Schmelzer, D. (2006). Selbstmanagement-Therapie (4. Auflage). Berlin: Springer.

Kaukua, J. (2004). Health-related quality of life in clinical weight loss studies. Dissertation, Universität Helsinki.

Kielmann, R. \& Herpertz, S. (2002). Möglichkeiten und Grenzen der Adipositas Chirurgie aus psychotherapeutischer Sicht. Verhaltenstherapie, 12 (4), 319-326.

King, A.C. Taylor, C.B., Haskell, W.L. \& Debusk, R.F. (1988). Strategies for increasing early adherence to and long-term maintenance of home-based exercise training in healthy middle-aged men and women. American Journal of Cardiology $61(8), 628-632$.

King, A.C., Frey-Hewitt, B., Dreon, D.M. \& Wood, P.D. (1989). Diet vs. exercise in weight maintenance. The effects of minimal intervention strategies on long-term outcomes in men. Archives of Internal Medicine; 149 (12), 2741-2746.

Kloss, S. (1993). Auswirkungen eines 4monatigen Bewegungsprogrammes auf das Selbstkonzept, die Körperwahrnehmung und das Bewegungsverhalten adipöser Frauen. Unveröff. Dipl. Arbeit Deutsche Sporthochschule, Köln.

Knapp, D.N. (1988). Behavioral management techniques and exercise promotion. In R.K. Dishman (Ed.), Exercise Adherence (pp. 203-236). Champaign, IL: Human Kinetics.

Kolotkin, R.L., Meter, K., \& Williams, G.R. (2001). Quality of life and obesity. Obesity Reviews, 2, 219-229.

Kolotkin, R.L., Crosby, R.D. \& Williams, G.R. (2002). Health-Related Quality of Life varies among obese subgroups. Obesity Research, 10 (2), 748-756.

Kossmann, B., Aidelsburger, P. \& Wasem, J. (2006). Adipositas - eine Krankheit? Explizite und implizite Kriterien für oder gegen die Definition von Adipositas als Krankheit auf Basis medizinischer Leitlinien Synopse nationaler und internationaler Leitlinien zur Diagnostik und Therapie der Adipositas. Zugriff am 20. Oktober 2006 unter http://www.uni-due.de/medman/Lehrstuhl/Aktuelles/060821_LL_Synopse_gesamt.pdf

Kuhn, D. (2001). Big Gym Fitness für Dicke. Marburg: Kilian.

Kushner, R.F. \& Foster, G. (2000). Obesity and quality of life. Nutrition, 16, 947952.

LaMonte, M.J., Ainsworth, B.E. \& Tudor-Locke, C. (2003). Assessment of physical activity and energy expenditure. In R.E. Andersen (Ed.), Obesity Etiology 
Assessment Treatment and Prevention (pp. 111-140). Champaign, IL: Human Kinetics.

Latifi, R., Kellum, J.M., De Maria, E.J. \& Sugerman, H.J. (2004). Surgical Treatment of obesity. In T.A. Wadden \& A.J. Stunkard (Ed.), Handbook of obesity Treatment (pp. 339-358). New York: Guilford Press.

Lauterbach, K., Hauner, H., Westenhöfer J. \& Wirth, A. (1998). Adipositas Leitlinie. Evidenz-basierte Leitlinie zur Behandlung der Adipositas in Deutschland. Zugriff am $20 \quad$ April unter http://www.westenhoefer.de/infos/adipositasleitlinie_expertenversion.pdf

Lee, I.M. \& Skerrett, P.J. (2001). Physical activity and all-cause mortality: what is the dose-response relation? Medicine \& Science in Sports \& Exercise, 33, 459-471.

Lehrke, S. \& Laessle, R.G. (2003). Adipositas. In U. Ehlert (Hrsg.), Lehrbuch der Verhaltensmedizin (S. 497 - 529). Heidelberg: Springer.

Lichtman, S.W., Pisarska, K., Berman, E.R., Pestone, M., Dowling, H., Offenbacher, E., Weisel, H., Heshka, S., Matthews, D.E.\& Heymsfield, S.B. (1992). Discrepancy between self-reported and actual caloric intake and exercise in obese subjects. New England Journal of Medicine, 327 (27), 1893-1898.

Lohman, T.G. (2002). Body composition. In C.G. Fairburn \& K.D. Brownell (Ed.), Eating Disorders and Obesity A Comprehensive Handbook (Second Edition) (pp. 6266). New York: Guilford Press.

Lombard, D.N., Lombard, T.N., \& Winett, R. A. (1995). Walking to meet health guidelines: The effect of prompting frequency and prompt structure. Health Psychology, 14 (2), 164-170.

Lombard, D.N. \& Lombard, T.N. (2003). Exercise Management of the obese Patient. In J. Foreyt, W.C. Poston, K. Mcinnis, J. Rippe (Ed.), Lifestyle Obesity Management (pp. 34-57). Massachusetts: Blackwell Publishing.

Long, B.C. (1993). A cognitive perspective on the stress reducing effects of physical exercise. In R. Seraganian (Ed.), Exercise Psychology: The Influence of Physical Exercise on Psychological Processes (pp. 339-357). New York: John Wiley \& Sons.

Levine, J.A., Eberhardt, N.L. \& Jensen, M.D. (1999). Role of nonexercise activity thermogenesis in resistance to fat gain in humans. Science, 283, 212-214.

McAuley, E., Bane, S. \& Mihalko, S.L. (1995). Exercise in middle-aged adults: Selfefficacy and self-presentational outcomes. Preventive Medicine, 24, 319-328.

Mannix, E.D., Dempsey, J.M., Engel, R.J., Schneider, B. \& Busk, M.F. (1999). The role of physical activity, exercise, and nutrition in the treatment of obesity. In D.J. Goldstein (Ed.), The Management of eating disorders and obesity (pp. 155-172). Totowa, New Jersey: Humana Press. 
Manson, J.E. Skerrett, P.J. \& Willett, W.C. (2002). Epidemiology of Health Risks associated with Obesity. In C.G. Fairburn \& K.D. Brownell (Ed.), Eating Disorders and Obesity A Comprehensive Handbook (Second Edition) (pp. 422-428). New York: Guilford Press.

Margraf, J. (2002). Spezielle Psychotherapie. Zugriff am 12 September 2004 unter http://www.ifap-index.de/bda-manuale/adipositas/therapie/psycho.html

Marlatt, G.A. (1995). Relapse: a cognitive-behavioral model. In C.G. Fairburn \& K.D. Brownell (Ed.), Eating Disorders and Obesity A Comprehensive Handbook (pp. 541-546). New York: Guilford Press.

Marlatt, G.A. \& Gordon, J.R. (Ed.). (1985). Relapse prevention. Maintenance Strategies in the Treatment of Addictive Behaviors New York: Guilford Press.

Mehnert, H. (2000). Typ-2-Diabetes (2. Auflage). München: Medikon Verlag.

Melanson, K.J., McInnis, K.J. \& Rippe, J.M. (2003). Modern Management of Obesity: The Value of a Multidisciplinary Approach. In J. Foreyt, W.C. Poston, K. Mcinnis, J. Rippe (Ed.), Lifestyle Obesity Management (pp. 1-33). Massachusetts: Blackwell Publishing.

Mensink, G.B.M. (1999). Körperliche Aktivität. Gesundheitswesen, 61 (Sonderheft 2), 126-131.

Mensink, G.B.M. (2003). Bundes-Gesundheitssurvey: körperliche Aktivität. Zugriff am $13 \quad$ May 2005 unter http://www.rki.de/cln_049/nn_199884/DE/Content/GBE/Gesundheitsberichterstattun $\mathrm{g} / \mathrm{GBEDownloadsB} /$ koerperliche_aktivitaeten,templateId=raw,property=publication File.pdf/koerperliche_aktivitaeten.pdf

Mensink, G.B.M., Lampert, T. \& Bergmann, E. (2005). Übergewicht und Adipositas in Deutschland 1984-2003. Bundesgesundheitsblatt - Gesundheitsforschung Gesundheitsschutz, 48, 1348-1356.

Miller, W.C., Koceja, D.M. \& Hamilton, E.J. (1997). A meta-analysis of the past 25 yearsof weight lossresearch using diet, exercise or diet plus exercise intervention. International Journal of obesity, 21 (10), 941-947.

Miller, K. \& Hell, E. (2002). Malabsorptionsmethoden in der bariatrischen Chirurgie. Zugriff am 10. Oktober 2004 unter http://www.fettsucht.at/Miller_Malabsorptionsmethoden_Zentralblatt.pdf

Müller, M.J., Mast, M., Bosy-Westphal, A. \& Danielzik, S. (2003). Diagnostik und Epidemiologie. In F. Petermann \& V. Pudel (Hrsg.), Übergewicht und Adipositas (S. 29-45). Göttingen: Hogrefe.

Munsch, S. (2002). Epidemiologie der Adipositas. Verhaltenstherapie, 12, 278-287. 
Munsch, S. \& Margraf, J. (2003). Prinzipien der Verhaltenstherapie der Adipositas. In F. Petermann \& V. Pudel (Hrsg.), Übergewicht und Adipositas (S. 223-238). Göttingen: Hogrefe.

National Heart, Lung, and Blood Institute (NHLBI), (1998). Clinical guidelines on the identification, evaluation, and treatment of overweight and obesity in adults: The Evidence report. Obesity Research, 6 (Suppl.) 51-210.

National Heart, Lung, and Blood Institute (NHLBI) \& North American Association for the Study of Obesity (NAASO), (2000). Practical Guide to the Identification, Evaluation and Treatment of Overweight and Obesity in Adults. Zugriff am 20. Oktober 2005 unter http://www.nhlbi.nih.gov/guidelines/obesity/prctgd_c.pdf

Paffenbarger, R.S. Jr, Hyde, R.T., Wing, A.L., Lee, I.M., Jung, D.L. \& Kampert, J.B. (1993). The association of changes in physical activity level and other lifestyle characteristics with mortality among men. New England Journal of Medicine, 328, 538-545.

Pate, R.R., Pratt, M., Blair, S.N., Haskell, W.L., Macera, C.A., Bouchard, C., Buchner, D., Ettinger, W., Heath, G.W., King, A.C. et al. (1995). Physical activity and public health: a recommendation from the Centers for Disease Control and Prevention and the American College of Sports Medicine. Journal of the American Medical Association, 273, 402-407.

Pavlou, K.N. Krey, S. \& Steffee, W.P. (1989). Exercise as an adjunct to weight loss and maintenance in moderately obese subjects. American Journal of clinical Nutrition, 49, 1115-1123.

Perri, M.G. (2002). Improving Maintenance in behavioral treatment. In C.G. Fairburn \& K.D. Brownell (Ed.), Eating Disorders and Obesity A Comprehensive Handbook (Second Edition) (pp. 593-598). New York: Guilford Press.

Pescatello, L.S. \& VanHeest, J.L. (2000). Physical activity mediates a healthier body weight in the presence of obesity. British Journal of Sports Medicine, 34, 86-93.

Petermann, F. \& Pudel, P. (Hrsg.). (2003). Übergewicht und Adipositas. Göttingen: Hogrefe.

Petermann, F. \& Häring, J. (2003). Verhaltenstherapeutische Methoden in der Adipositastherapie. In F. Petermann \& V. Pudel (Hrsg.), Übergewicht und Adipositas (S. 238-262). Göttingen: Hogrefe.

Petermann, F. \& Winkel, S. (2003). Selbstkonzept und Körperbild bei Menschen mit Adipositas. In F. Petermann \& V. Pudel (Hrsg.), Übergewicht und Adipositas (S. 127-152). Göttingen: Hogrefe.

Plante, T.G. \& Rodin, J. (1990). Physical fitness and enhanced psychological health. Current Psychology: Research and Reviews, 9, 3-24. 
Platte, P. (2003). Der Energiestoffwechsel. In F. Petermann \& V. Pudel (Hrsg.), Übergewicht und Adipositas (S. 47-55). Göttingen: Hogrefe.

Pi-Sunyer, F.X. (1999). Medical consequences of obesity. In D.J. Goldstein (Ed.), The Management of eating disorders and obesity (pp. 84-92). Totowa, New Jersey: Humana Press.

Pories, W.J. \& Beshay, J.E. (2002). Surgery for obesity: Procedures and weight loss. In C.G. Fairburn \& K.D. Brownell (Ed.), Eating Disorders and Obesity A Comprehensive Handbook (Second Edition) (pp. 562-567). New York: Guilford Press.

Prentice, A.M. \& Jebb, S.A. (1995). Obesity in Britain: Gluttony or sloth? British Medical Journal, 311, 437-439.

Prentice, A.M. \& Jebb, S.A. (2000). Physical activity level and weight control in adults. In C. Bouchard (Ed.), Physical Activity and Obesity (pp. 247-261). Champaign, IL: Human Kinetics.

Prentice, A.M. (2002). Measurement of energy expenditure. In C.G. Fairburn \& K.D. Brownell (Ed.), Eating Disorders and Obesity A Comprehensive Handbook (Second Edition) (pp. 131-135). New York: Guilford Press.

Prochaska, J.O. \& DiClemente, C.C. (1983). Stages and processes of self-change of smoking: towards an integrative model of change. Journal of Consulting Clinical Psychology, 51, 390-395.

Pudel, V. (1998). Psychologische Aspekte der Adipositas - Prävention, Therapie und Gewichtserhaltung. In J.G. Wechsler (Hrsg.), Adipositas Ursachen und Therapie (S. 200-214). Berlin: Blackwell.

Puhl, R. \& Brownell, K.D. (2002). Stigma, Discrimination, and Obesity. In C.G. Fairburn \& K.D. Brownell (Ed.), Eating Disorders and Obesity A Comprehensive Handbook (Second Edition) (pp. 108-112). New York: Guilford Press.

Rathmanner, T., Meidlinger, B., Baritsch, C., BEng, K.L., Dorner, T. \& Kunze, M. (2006). Erster Österreichischer Adipositasbericht 2006. Zugriff am 5 Februar 2007 unter http://www.adipositas-austria.org/pdf/3031_AMZ_Adipositas_3108_final.pdf

Raynor, D.A., Coleman, K.J., \& Epstein, L.H. (1998). Effects of proximity on the choice to be physically active or sedentary. Research Quarterly for Exercise and Sport, 69, 99-103.

Rodin, J. Silberstein, L.R. \& Striegel-Moore, R.H. (1985). Women and weight: A normative discontent. In T.B. Sondereggar (Ed.), Nebraska Symposium on Motivation: Psychology and gender (pp. 267-307). Lincoln: University of Nebraska Press. 
Rosen, J.C. (2002). Obesity and body image. Obesity and Quality of life. In C.G. Fairburn \& K.D. Brownell (Ed.), Eating Disorders and Obesity A Comprehensive Handbook (Second Edition) (pp. 399-402). New York: Guilford Press.

Rosen, J.C. (2003). Improving body image in obesity. In J.K. Thompson (Ed.), Body Image, Eating Disorders, and Obesity (pp. 425-440). Washington, DC: American Psychological Association.

Rost, R., Heck, H. \& Hollmann, W. (o.J.). Die Fahrradergometrie in der Praxis (2. Aufl.). o.O.: Bayer.

Salbe, A.D. \& Ravussin, E. (2000). The Determinants of Obesity. In C. Bouchard (Ed.), Physical Activity and Obesity (pp. 69-102). Champaign, IL: Human Kinetics.

Sallis, J.F., Hovell, M.F., Hofstetter, C.R., Elder, J.P., Caspersen, C.J., Hackley, M., \& Powell, K.E. (1990). Distance between homes and exercise facilities related to the frequency of exercise among San Diego residents. Public Health Reports, 105, 179185.

Sallis, J.F. \& Owen, N. (1999). Physical activity \& behavioural medicine. Thousand Oaks: Sage Publication.

Sallis, J.F. \& Zabinski, M.F. (2002). measurement of physical activity. In C.G. Fairburn \& K.D. Brownell (Ed.), Eating Disorders and Obesity A Comprehensive Handbook (Second Edition) (pp. 136-140). New York: Guilford Press.

Saris, W.H. (2002). Metabolic Effects of exercise in overweight individuals. In C.G. Fairburn \& K.D. Brownell (Ed.), Eating Disorders and Obesity A Comprehensive Handbook (Second Edition) (pp. 495-499). New York: Guilford Press.

Saris, W.H., Blair, S.N., van Baak, M.A., Eaton, S.B., Davies, P.S., Di Pietro, L., Fogelholm, M., Rissanen, A., Schoeller, D., Swinburn, B., Tremblay, A., Westerterp, K.R. \& Wyatt, H. (2003). How much physical activity is enough to prevent unhealthy weight gain? Outcome of the IASO 1st Stock Conference and consensus statement. Obesity Reviews, 4 (2), 101-114.

Sarwer, D.B., Wadden, T.A. \& Foster, G. (1998). Assessment of body dissatisfaction in obese women: Specificity, severity and clinical significance. Journal of consulting and clinical psychology, 66, 651-654.

Sarwer, D.B., Wadden, T.A., Pertschuk, M.J. \& Whitaker, L.A. (1998). The psychology of cosmetic surgery: A review and reconceptualization. Clinical Psychology Review, 18, 1-22.

Sarwer, D.B. \& Thompson, J.K. (2004). Obesity and body image disturbance. In T.A. Wadden \& A.J. Stunkard (Ed.), Handbook of obesity Treatment (pp. 447-464). New York: Guilford Press. 
Schmidt, F., Deimel, H., Alexandridis, J., Alexandridis, K., Ehrig, C. \& Cuntz, U. (2000). Bewegungstherapeutische Maßnahmen bei Adipositas. Gesundheitssport und Sporttherapie, 16 (6), 217-221.

Schneider, R. (1996). Relevanz und Kosten der Adipositas in Deutschland. Ernährungs-Umschau, 43 (10), 369-374.

Schüle, K. \& Schnieders, S. (2004). Indikationskatalog. In K. Schüle \& G. Huber (Hrsg.), Grundlagen der Sporttherapie (2. Auflage) (S. 301-324). München: Elsevier.

Schulz, L.O. \& Schoeller, D.A. (1994). A compilation of total daily energy expenditures and body weights in healthy adults. American Journal of Clinical Nutrition, 60, 676-681.

Schutz, Y., Weinsier, R.L. \& Hunter, G.R. (2001). Assessment of free-living physical activity in humans: An overview of currently available and proposed new measures. Obesity research, 9 (6), 368-379.

Seidell, J.C. \& Tijhuis, M.A.R. (2002). Obesity and Quality of life. In C.G. Fairburn \& K.D. Brownell (Ed.), Eating Disorders and Obesity A Comprehensive Handbook (Second Edition) (pp. 388-392). New York: Guilford Press.

Simkin, L.R., \& Gross, A.M. (1994). Assessment of coping with high-risk situations for exercise relapse among healthy women. Health Psychology, 13, 274-277.

Sørensen, M., Anderssen, S., Hjerman, I., Holme, I. \& Ursin, H. (1997). Exercise and diet interventions improve perceptions of self in middle-aged adults. Scandinavian Journal of Medicine Science in Sports, 7, 312-320.

Staffieri, J.R. (1967). A study of social stereotype of body image in children. Journal of Personality and Social Psychology, 7, 101-104.

Stunkard, A.J., (1959). Eating patterns and obesity. Psychiatric Quarterly, 33, 284294.

Stunkard, A.J.\& Mendelson, M. (1961). Disturbances in body image of some obese persons. Journal of the American Dietetic Association, 38 (4), 328-331.

Stunkard, A.J. \& Mendelson, M. (1967). Obesity and body image: I. Characteristics of disturbances in the body image of some obese Persons. American Journal of Psychiatry, 123, 1296-1300.

Stunkard, A.J. (2004). Bing-Eating Disorder and the Night-Eating Syndrome. In T.A. Wadden \& A.J. Stunkard (Ed.), Handbook of obesity Treatment (pp. 107-121). New York: Guilford Press.

Sundquist, J. \& Johansson, S.E. (1998). The influence of socioeconomic status, ethnicity and lifestyle on body mass index in a longitudinal study. International Journal of Epidemiology, 27, 57-63. 
Tataranni, P.A. \& Ravussin, E. (2004). Energy metabolism and obesity. In T.A. Wadden \& A.J. Stunkard (Ed.), Handbook of obesity Treatment (pp. 42-72). New York: Guilford Press.

Telch, C. \& Agras, S. (1994). Obesity, binge eating and psychopathology: Are they related? International Journal of Eating Disorders, 1, 53-61.

Thompson, D. \& Wolf, A.M. (2001).The medical-care cost burden of obesity. Obesity Reviews, 2 (3), 189-197.

Thompson, J.K. \& Tantleff-Dunn, S. (1998). Assessment of body image disturbance in obesity. Obesity Research, 6, 375-377.

Thompson, J.K. (2003). Introduction: Body image, eating disorders, and obesity -an emerging synthesis. In J.K. Thompson (Ed.), Body Image, Eating Disorders, and Obesity. (pp. 1-20). Washington, DC: American Psychological Association.

Thompson, J.K. (Ed.). (2003). Body Image, Eating Disorders, and Obesity. Washington, DC: American Psychological Association.

Thompson, J.L., Manore, M.M. \& Thomas, J.R. (1996). Effects of diet and diet-plus exercise programs on resting metabolic rate: a meta-analysis. International Journal of Sport Nutrition, 6, 41-61.

Toplak, H. (2003). Aktuelle Aspekte der Pharmakotherapie der Adipositas. Journal für Kardiologie, 10 (10), 424-426. Zugriff am 24. Februar 2004 unter http://www.kup.at/kup/pdf/3645.pdf

Trappe, H.J. \& Löllgen, H. (2000). Leitlinien zur Ergometrie herausgegeben vom Vorstand der deutschen Gesellschaft für Kardiologie - Herz und Kreislaufforschung, bearbeitet im Auftrag der Kommission für klinische Kardiologie. Zeitschrift für Kardiologie, 89, 821-837.

Tuschhoff, T., Benecke-Timp, A. \& Vogel, H. (1995). Adipositas. In F. Petermann (Hrsg.), Verhaltensmedizin in der Rehabilitation (S. 337-370). Göttingen: Hogrefe.

Ungerer-Röhrig, U. (2001). Neustrukturierung des Dortmunder Fragebogens zur Bewegungstherapie. Gesundheitssport und Sporttherapie, 17, 187-188.

U.S. Departement of Health and Human Services. (1996). Physical Activity and Health : A report of the Surgeon General. Atlanta, GA: Centers for Disease Control and Prevention.

U.S. Departement of Health and Human Services. (2005). Dietary Guidelines for Americans. Dietary Guidelines Advisory Committee Report. Zugriff am 20 December 2005 unter http://www.health.gov/dietaryguidelines/dga2005/report/HTML/G1_Glossary.htm 
Valdez, R. \& Williamson, D.F. (2002). Prevalence and demographics of obesity. In C.G. Fairburn \& K.D. Brownell (Ed.), Eating Disorders and Obesity A Comprehensive Handbook (Second Edition) (pp. 417-421). New York: Guilford Press.

Vanden-Abeele, J. \& Schüle, K. (2004). Wissenschaftliche Begründung der Sporttherapie aus dynamischer und handlungsorientierter Sicht. In K. Schüle \& G. Huber (Hrsg.), Grundlagen der Sporttherapie (2. Auflage) (S. 7-37). München: Elsevier.

Vögele, C. (2003). Sport und Bewegung als Behandlungsansatz. In F. Petermann \& V. Pudel (Hrsg.), Übergewicht und Adipositas (S. 283-302). Göttingen: Hogrefe.

Völker, K. Lagerstrøm, D. \& Matheis, L. (1985). Einfluss einer 8 wöchigen Bewegungstherapie auf die Gewichtsabnahme und die Leistungsfähigkeit adipöser Frauen und Männer. Die Medizinische Welt, 36, 72-76.

Votruba, S.B., Horvitz, M.A. \& Schoeller, D.A. (2000). The role of exercise in the treatment of obesity. Nutrition; 16, 179-188.

Wadden, T.A. \& Sarwer, D.B. (1999). Behavioral treatment in obesity: new approaches to an old disorder. In D.J. Goldstein (Ed.), The Management of Eating disorders and Obesity (pp. 173-200). Totowa, New Jersey: Humana Press.

Wadden, T.A. \& Phelan, S. (2002). Assessment of quality of life in obese individuals. Obesity Research, 10 (1), 50-57.

Wadden, T.A. \& Phelan, S. (2004). Behavioral assessment of the obese patient. In T.A. Wadden \& A.J. Stunkard (Ed.), Handbook of obesity treatment (pp. 186-226). New York: Guilford Press.

Wadden, T.A., Womble, L.G., Stunkard A.J. \& Anderson, D.A. (2004). Psychological Consequences of Obesity and Weight Loss. In T.A. Wadden \& A.J. Stunkard (Ed.), Handbook of obesity treatment (pp. 144-169). New York: Guilford Press.

Wadden, T.A., Butryn, M.L. \& Byrne, K.J. (2004). Efficacy of lifestyle modification for long-term weight control. Obesity Research, 12 (Suppl.), 151-162

Wadden, T.A. \& Stunkard, A.J. (Ed.). (2004). Handbook of obesity treatment. New York: Guilford Press.

Wagner, K. (2005). nTOBEC: eine neue Methode zur Erfassung der Körperzusammensetzung. Dissertation, Universität Potsdam.

Wallace, J.P., Raglin, J.S. \& Jastremski, C. (1995). Twelve month adherence of adults who joined a fitness program with a spouse vs. without a spouse. Journal of Sports Medicine and Physical Fitness, 35, 206-213. 
Weinsier, R.L. \& Kushner, R.F. (2002). Clinical assessment of obese patients. In C.G. Fairburn \& K.D. Brownell (Ed.), Eating Disorders and Obesity A Comprehensive Handbook (Second Edition) (pp. 512-517). New York: Guilford Press.

Wilhelm, M., Strütt-Neeb, P., Opielka, C. \& Cuntz, U. (2002). Verhaltenstherapeutische Ernährungstherapie bei Adipositas. Verhaltenstherapie, 12 (4), 311-318.

Willamson, D.F., Madans, J., Anda, R.F., Kleinman, J.C., Kahn, H.S. \& Byers, T. (1993) Recreational physical activity and ten-year weight change in a US national cohort. International Journal of Obesity, 17, 279-286.

Williamson, D.F. (1996). Dietary intake and physical activity as predictors of weight gain in observational, prospective studies of adults. Nutrition Reviews, 54, 101-109.

Willimczik, K. (1997). Forschungsmethoden in der Sportwissenschaft, Bd. 1. Statistik im Sport. Grundlagen - Verfahren - Anwendungen. mit ausführlich kommentierten SPSS- Ausdrucken (3. überarbeitete Aufl.). Hamburg: Czwalina.

Wilson, G.T. \& Brownell, K.D. (2002). Behavioral treatment for obesity. In C.G. Fairburn \& K.D. Brownell (Ed.), Eating Disorders and Obesity A Comprehensive Handbook (Second Edition) (pp. 524-528). New York: Guilford Press.

Wing, R.R. (1999). Physical activity in the treatment of the adulthood overweight and obesity: current evidence and research issues. Medicine \& Science in Sports \& Exercise, 21 (11, Suppl.), 547-552.

Wing, R.R. \& Jakicic, J.M. (2000). Changing lifestyle: Moving from sedentary to active. In C. Bouchard (Ed.), Physical Activity and Obesity (pp. 372-389). Champaign, IL: Human Kinetics.

Wing, R.R. \& Hill, J.O. (2001). Successful weight loss maintenance. Annual Review of Nutrition, 21, 323-341.

Wing, R.R. (2004). Behavioral weight control. In T.A. Wadden \& A.J. Stunkard (Ed.), Handbook of obesity Treatment (pp. 301-316). New York: Guilford Press.

Wing, R.R. \& Phelan, S. (2005). Long-term weight loss maintenance. American Journal of Clinical Nutrition; 82 (suppl.), 222-225.

Wirth, A. (2000). Adipositas. Epidemiologie, Ätiologie, Folgekrankheiten, Therapie (2. überarbeitete und erweiterte Aufl.). Berlin: Springer.

Wirth, A. (2003). Adipositas- Fibel (2., vollständig überarbeitete Auflage). Berlin: Springer.

Wirth, A. (2003). Adipositas -assoziierte Krankheiten. In F. Petermann \& V. Pudel (Hrsg.), Übergewicht und Adipositas (S. 105-126). Göttingen: Hogrefe. 
Wolf, A.E. (2002). The Health Economics of Obesity and Weight Loss. In C.G. Fairburn \& K.D. Brownell (Ed.), Eating Disorders and Obesity A Comprehensive Handbook (Second Edition) (pp. 453-459). New York: Guilford Press.

Woll, A. (2004). Diagnose körperlich-sportlicher Aktivität, Fitness und Gesundheit: Methodenband I. dissertation.de - Verlag im Internet GmbH.

Woll, A. \& Bös, K. (2004). Wirkungen von Gesundheitssport. Bewegungstherapie und Gesundheitssport, 20, 1-10.

Wood, P.D., Stefanick, M.L., Dreon, D.M., Frey-Hewitt, B., Garay, S.C., Williams, P.T., Superko, H.R., Fortmann, S.P., Albers, J.J., Vranizan, K.M., et al., (1988). Changes in plasma lipids and lipoproteins in overweight men during weight loss through dieting as compared with exercise. New England Journal of Medicine, 319, 1173-1179.

Wood, P.D., Stefanick, M.L., Williams, P.T. \& Haskell, W.L. (1991). The effects on plasma lipoproteins of a prudent weight-reduced diet, with or without exercise, in overweight men and women. New England Journal of Medicine, 325, 461-466.

World Health Organization [WHO] (2000). Obesity: Preventing and managing the global epidemic. Report of a WHO consultation. WHO Technical Report Series, 894. Zugriff am 20. April 2005 unter http://whqlibdoc.who.int/trs/WHO_TRS_894.pdf

World Health Organization [WHO] (2003). Obesity and Overweight. Global Strategy on Diet, Physical Activity and Health. Zugriff am 25. April 2005 unter http://www.who.int/hpr/NPH/docs/gs_obesity.pdf

World Health Organization [WHO] (2005). Adipositas: Eine Herausforderung für die Europäische Region der WHO. Faktenblatt EURO/13/05. Zugriff am 15. Dezember 2006 unter http://www.euro.who.int/document/mediacentre/fs1305g.pdf

World Health Organization [WHO] (2006). Obesity and overweight. Fact sheet $N^{\circ} 311$. Zugriff am $15 . \quad$ Dezember 2006 unter http://www.who.int/mediacentre/factsheets/fs311/en/index.html

World Health Organization [WHO] (2006). Internationale Statistische Klassifikation der Krankheiten und verwandter Gesundheitsprobleme 10. Revision Version 2006. Zugriff am $20 . \quad$ April 2005 unter http://www.dimdi.de/dynamic/de/klassi/diagnosen/icd10/htmlamtl2006/fr-icd.htm

Woweries, J. (2004). Bewegung/körperliche Aktivität/Sport als Behandlungsprinzip bei Adipositas im Kindes- und Jugendalter. Deutsche Zeitschrift für Sportmedizin, 55 (4), 6-7.

www.adipositas-online.de. (2006). Fachinfos. 5. Verhaltenstherapeutische Komponenten. Zugriff am 11 November 2006 unter http://www.adipositasonline.de/diaet-abnehmen-12.htm 
Wydra, G. (2004). Problemorientierte Diagnosestrategie für die Sporttherapie. In K. Schüle \& G. Huber (Hrsg.), Grundlagen der Sporttherapie (2. Auflage) (S. 99-108). München: Elsevier.

Yalom, I.D. (1989). The theory and practice of group psychotherapy. New York: Basic Books.

Yeung, R.R. (1996). The acute effects of exercise on mood state. Journal of Psychosomatic Research 40, 123-141. 
10 Anhang 
Flussdiagramm,Adipositasprävention und -therapie“ DAC 2006

\begin{tabular}{|c|c|c|}
\hline $\begin{array}{l}\text { Grad des Körpergewichts } \\
\text { und der Gesundheitsgefährdung }\end{array}$ & Ziel & Maßnahmen \\
\hline Normalgewicht (BMI 18,5 - 24,9) & Gewichtsstabilisierung & Ggf. Gewichtsmonitoring \\
\hline $\begin{array}{l}\text { Normalgewicht (BMI } 18,5-24,9 \text { ) } \\
\text { plus Risikofaktor und/oder Komorbiditäten }\end{array}$ & $\begin{array}{l}\text { Gewichtsstabilisierung, bei familiärer } \\
\text { Prädisposition Gewichtszunahme }>3 \mathrm{~kg} \text { ver- } \\
\text { hindern. Risikofaktoren-Management, } \\
\text { z.B. Aufgabe des Rauchens, gesunder Lebensstil }\end{array}$ & $\begin{array}{l}\text { Gewichtsmonitoring, Risikofaktoren- } \\
\text { Management, Therapie der Komorbiditäten, } \\
\text { Beratung über gesundheitsförderlichen Lebensstil }\end{array}$ \\
\hline Präadipositas (BMI 25 - 29,9) & Verhinderung einer Gewichtszunahme & $\begin{array}{l}\text { Gewichtsmonitoring, } \\
\text { Beratung über gesundheitsförderlichen Lebensstil }\end{array}$ \\
\hline $\begin{array}{l}\text { Präadipositas (BMI } 25-29,9 \text { ) } \\
\text { plus Risikofaktor und/oder Komorbiditäten } \\
\text { oder Taillenumfang w: }>80 \mathrm{~cm} \mathrm{m:}>94 \mathrm{~cm}\end{array}$ & Dauerhafte Gewichtsreduktion um $5-10 \%$ & $\begin{array}{l}\text { Basisprogramm*, Risikofaktoren- } \\
\text { Management, Therapie der Komorbiditäten, bei } \\
\text { BMI }>27 \mathrm{~kg} / \mathrm{m}^{2} \text { frühestens nach } 12 \text { wöchiger Therapie } \\
\text { zusätzliche medikamentöse Therapie erwägen }\end{array}$ \\
\hline Adipositas Grad I (BMI $30-34,9$ ) & Dauerhafte Gewichtsreduktion um 5-10\% & $\begin{array}{l}\text { Basisprogramm * } \\
\text { Beratung über gesundheitsförderlichen Lebensstil }\end{array}$ \\
\hline $\begin{array}{l}\text { Adipositas Grad I (BMI } 30-34,9 \text { ) } \\
\text { plus Risikofaktor und/oder Komorbiditäten } \\
\text { oder Taillenumfang w: }>88 \mathrm{~cm} \mathrm{m:}>102 \mathrm{~cm}\end{array}$ & Dauerhafte Gewichtsreduktion um 5-10\% & $\begin{array}{l}\text { 1.Basisprogramm *, Risikofaktoren- } \\
\text { Management, Therapie der Komorbiditäten } \\
\text { 2. Wenn kein Erfolg, frühestens nach } \\
\text { 12 Wochen zusätzliche medikamentöse } \\
\text { Therapie erwägen }\end{array}$ \\
\hline Adipositas Grad II (BMI $35-39,9)$ & $\begin{array}{l}\text { Dauerhafte Gewichtsredultion um } \\
\geq 10 \%\end{array}$ & $\begin{array}{l}\text { Basisprogramm * } \\
\text { Beratung über gesundheitsförderlichen Lebensstil }\end{array}$ \\
\hline $\begin{array}{l}\text { Adipositas Grad II (BMI } 35-39,9 \text { ) } \\
\text { plus Risikofaktor und/oder Komorbiditäten }\end{array}$ & $\begin{array}{l}\text { Dauerhafte Gewichtsreduktion um } \\
10-20 \%\end{array}$ & $\begin{array}{l}\text { 1. Basisprogramm *, Risikofaktoren- } \\
\text { Management, Therapie der Komorbiditäten } \\
\text { 2.wenn kein Erfolg, frühestens nach } 12 \text { Wochen } \\
\text { zusätzliche medikamentöse Therapie erwägen } \\
\text { 3.bei erfolgloser konservativer Therapie } \\
\text { chirurgische Maßnahmen erwägen }\end{array}$ \\
\hline Adipositas Grad III (BMI > 40) & Dauerhafte Gewichtsreduktion um $10-30 \%$ & $\begin{array}{l}\text { 1.Basisprogramm *, Risikofaktoren- } \\
\text { Management, Therapie der Komorbiditäten } \\
\text { 2.wenn kein Erfolg, frühestens nach } 12 \text { Wochen } \\
\text { zusätzliche medikamentöse Therapie erwägen } \\
\text { 3. bei erfolgloser konservativer Therapie } \\
\text { chirurgische Therapie erwaggen }\end{array}$ \\
\hline
\end{tabular}

* Das Basisprogramm setzt sich zusammen aus Ernährungstherapie(Kapitel 6.4.2), Bewegungstherapie (Kapitel 6.4.3) und Verhaltensmodifikation (Kapitel 6.4.4). 


\section{Presseinformation \\ Erschienen am 16 April 2003 im „Oberbayrischen Volksblatt“}

Bewegung hilft bei starkem Übergewicht

Ein ambulantes Bewegungsprogramm für Männer und Frauen mit starkem Übergewicht in der Medizinisch-Psychosomatischen Klinik Roseneck.

Die überragende Bedeutung von körperlicher Aktivität und Sport im Vergleich zur rein diätetischen Behandlungsansätze in der Behandlung des Übergewichts wird durch zahlreiche Forschungsergebnisse der letzten Jahre immer deutlicher. Körperliche Aktivität führt zu einer Zunahme an Muskelmasse und damit zu einem gesteigerten Energieverbrauch. Die häufig erhöhten Blutzuckerwerte werden dabei normalisiert, der Blutdruck sinkt - damit wird nicht nur das Körpergewicht selbst, sondern vor allem auch die negativen Auswirkungen des Übergewichts auf die Gesundheit vermindert

Viele der Betroffenen wissen das auch schon lange. Das Kernproblem liegt offensichtlich in der Problematik, das eigene Wissen in tägliche körperlicher Aktivität umzusetzen.

In der Klinik Roseneck werden seit Jahren Patienten mit krankhaftem Übergewicht behandelt. Durch intensive eigene Forschung im Rahmen der stationären Behandlung wurde ein Bewegungsprogramm in der Klinik entwickelt und mittlerweile über Jahre erprobt, das Übergewichtige erfolgreich dabei unterstützt, neue und für sie angemessene Bewegungsgewohnheiten aufzubauen.

Dieses Programm soll nun im Rahmen eines ambulanten Forschungsprojektes auch Betroffenen der Umgebung Prien zugute kommen. Ziel ist es daher, Übergewichtige so zu motivieren, dass ihnen der Spaß an der Bewegung vermittelt wird ohne sich dabei zu überfordern.

Walking, Wassergymnastik, aber auch Körperwahrnehmungs- und Entspannungsübungen gehören genauso zu den Inhalten wie Informationen über Bewegung in Alltag und Freizeit.

Durch die regelmäßige Bewegung wird eine Verbesserung der Leistungsfähigkeit erreicht. Es wird gezeigt wie Fitness, Lust und Freude an der Bewegung und ein beschwerdefreierer Umgang mit dem Körper auch bei starkem Übergewicht möglich sind.

Das Programm ist intensiv und wird sportwissenschaftlich betreut. Der individuelle Erfolg wird durch sportmedizinische Testungen belegt. Die Teilnehmer erhalten genaue Informationen über den Verlauf gesundheitsrelevanter Parameter wie Ausdauer, Kraft und Körperzusammensetzung.

Durch eine weitere Testung 6 Monate nach Kursende werden die Effekte des Programms weiter verfolgt. 
Voraussetzung für eine Kursteilnahme ist ein Übergewicht mit einem Body- Mass Index (BMI) von mindestens $35 \mathrm{Kg} / \mathrm{m}^{2}$.

Den Body- Mass- Index (BMI) berechnet man mit der Formel Körpergewicht in Kg geteilt durch Körpergröße mal Körpergröße.

Beispiel: $\mathrm{BMI}=95 \mathrm{Kg} /(1,60 \mathrm{~m} X 1,60 \mathrm{~m})=35 \mathrm{~kg} / \mathrm{m}^{2}$

Der Kostenbeitrag für die Teilnahme beträgt 50 Euro (100 DM). Bei Abschluss des gesamten Kurses werden von den Krankenkassen $80 \%$ des Unkostenbeitrages an die Teilnehmer rückerstattet.

Der Kurs findet in den Räumen der Klinik Roseneck statt. Er läuft über acht Wochen bei 2 - 3 Termine wöchentlich. Wenn Sie an einer Teilnahme interessiert sind, wenden Sie sich über die Klinik Roseneck an die Kontaktperson Herrn Jannis Alexandridis, Sporttherapeut (M.A. EMDAPA) Abteilung für Sport- und Bewegungstherapie Tel. 08051/96 29 12, $08051 / 682009$ oder email: jannis.alexandridis@arcormail.de 


\section{Brief an die niedergelassenen Praxen}

\section{Ankündigung eines ambulanten Bewegungsprogramms für Männer und Frauen mit starkem Übergewicht in der Klinik Roseneck}

Sehr geehrter Herr Dr./Fr. Dr.

mit diesem Schreiben möchten wir Sie über ein ambulantes sporttherapeutisches Adipositasprogramm informieren, das künftig in der Klinik Roseneck in Prien am Chiemsee in Kooperation mit der Universität Heidelberg stattfinden wird.

Bei diesem Adipositasprojekt handelt es sich um eine sportwissenschaftliche Evaluationsstudie, die die Effekte eines speziellen Bewegungsprogramms für übergewichtige Menschen (Adipositas 2. Grades) untersucht. Eine genaue Beschreibung des Projektes finden Sie auf den beiliegenden Seiten.

Wir möchten anfragen, ob Sie an einer Zusammenarbeit Interesse haben und für unser Projekt geeignete Patienten aus Ihrer Praxis empfehlen können?

Betreut wird dieses Projekt von Herrn Jannis Alexandridis, der seit mehreren Jahren als Sporttherapeut (Sportstudium, Sport in Prävention und Rehabilitation) in der Klinik Roseneck mit stark übergewichtigen Patienten arbeitet. Er würde sich in den nächsten Tagen bei Interesse gerne telefonisch oder persönlich bei Ihnen vorstellen.

Das Projekt wird durch die gesetzlichen Krankenkassen unterstützt (Übernahme der Kursgebühren von ca. 100 DM bei regelmäßiger Teilnahme).

Über eine Kooperation mit Ihrer Praxis würden wir uns sehr freuen.

Für weitere Fragen und Rückmeldungen stehen wir Ihnen jederzeit gern zur Verfügung.

Kontaktperson:

Mit freundlichen Grüssen
Jannis Alexandridis, Medizinisch-Psychosomatische Klinik Roseneck Abteilung Sporttherapie

Am Roseneck 6, 83209 Prien am Chiemsee

Tel. 08051/682009

Email: jannis.alexandridis@arcormail.de 


\section{Brief an die Krankenkassen}

An die BEK

Geschäftsstelle Rosenheim

Herrn

Prien, den 28.11.01

\section{Anfrage wegen der Förderung eines ambulanten Bewegungsprogramms für Männer und Frauen mit starkem Übergewicht in der Klinik Roseneck durch die BEK}

Sehr geehrter Herr

ein ambulantes sporttherapeutisches Adipositasprogramm soll künftig in der Klinik Roseneck in Prien am Chiemsee in Kooperation mit der Universität Heidelberg stattfinden.

Bei diesem Adipositasprojekt handelt es sich um eine sportwissenschaftliche Evaluationsstudie, die die Effekte eines speziellen Bewegungsprogramms für übergewichtige Menschen (Adipositas 2. Grades) untersucht. Eine genaue Beschreibung des Projektes finden Sie auf den beiliegenden Seiten.

Ziel des Projekts ist die Entwicklung und Implementierung neuer und effektiverer Therapieansätze in der Adipositasbehandlung.

Im Rahmen der Untersuchung wird übergewichtigen Menschen aus Prien und Umgebung eine sehr wichtige ambulante sporttherapeutische Behandlung geboten. Bewegungsbezogene Maßnahmen dienen der Gesundheitsförderung und können als Präventivstrategie Folgeschäden wie -kosten verringern helfen.

Mit diesem Schreiben möchte ich anfragen, ob seitens der BEK die Möglichkeit besteht, die ambulante sporttherapeutische Adipositasbehandlung zu unterstützen. Dieses ambulante Projekt betrifft keine stationär in der Klinik Roseneck behandelte Patienten.

1. Die BEK könnte uns bei der Patientenrekrutierung behilflich sein.

Meinen Sie, dass es möglich wäre, dass Sie interessierte BEK Patienten mit Adipositas in diesem Programm schicken könnten?!

2. Zum anderen möchten wir anfragen, ob und unter welchen Bedingungen der aus motivationalen und therapeutischen Gründen geforderte Eigenbeitrag (von ca. $100 \mathrm{DM}$ ) von der Barmer Ersatzkasse übernommen werden könnte?

Über eine Kooperation mit der BEK würden wir uns sehr freuen.

Für weitere Fragen und Rückmeldungen stehe ich Ihnen jederzeit gern zur Verfügung. Kontaktperson: Jannis Alexandridis, Klinik Roseneck, Abteilung Sporttherapie, Am Roseneck 6, 83209 Prien am Chiemsee, Tel. 08051/682009

Mit freundlichen Grüssen 


\section{Einverständniserklärung}

Evaluationsstudie

Zur Wirksamkeit des ambulanten Bewegungsprogramms

für Männer und Frauen mit starkem Übergewicht

(Name, Vorname des Teilnehmers)

Sie haben sich für das ,ambulante Bewegungsprogramm für Männer und Frauen mit starkem Übergewicht" , das in der Klinik Roseneck stattfindet angemeldet.

Das Programm hat das Ziel, die Wirksamkeit der ambulanten bewegungstherapeutischen Maßnahmen im bezug auf das Übergewicht zu überprüfen.

Die Teilnahme an diesem Bewegungsprogramm beinhaltet zusätzlich das Ausfüllen von Fragebögen und die Teilnahme an sportmotorischen Tests vor und nach dem Kurs:

Testzeitpunkte sind:

4 Wochen vor Kursbeginn Fragebogenmappe,

sportmotorische Fitnesstests

Körperfettanteilbestimmung

bei Kursbeginn $\quad$ Fragebogenmappe,

sportmotorische Fitnesstests

Körperfettanteilbestimmung

bei Kursende

Fragebogenmappe, sportmotorische Fitnesstests Körperfettanteilbestimmung

6 Monate nach Kursende Fragebogenmappe, sportmotorische Fitnesstests Körperfettanteilbestimmung

Mir wurde versichert, dass meine sämtlichen Angaben streng vertraulich behandelt werden, nicht an Dritte weitergegeben werden und grundsätzlich der Schweigepflicht unterliegen. Die Auswertung der Ergebnisse erfolgt anonymisiert; Rückschlüsse auf einzelne Personen sind z.B. bei späteren wissenschaftlichen Veröffentlichungen nicht möglich.

Ich bin über Sinn und Zweck des Programms informiert worden und ich habe Gelegenheit gehabt, Fragen zu stellen und klärende Antworten zu bekommen.

Ich erkläre mich damit einverstanden, an dieser Studie teilzunehmen

(Ort, Datum)

(Unterschrift des

Teilnehmers) 


\section{Persönlicher Fragebogen/Screening}

Name:

Geschlecht :

Telefon:1)

2)

Straße:

PLZ/Wohnort:

Gewicht /BMI

Geburtsdatum:

Familienstand :

Beruf

Raucher $\square$ nicht Raucher

Behandelnder Arzt

Krankenkasse

Flyer

Bekannter

Zeitung

Allgemeiner Gesundheitszustand (Screening- Eingangsdiagnose)

- Erhöhter Blutzuckerspiegel bekannt/Zuckerkrankheit Ja ( )Nein ( )

- Lungenkrankheit ?.........

- Sind Sie Herzkrank? Ja ( ) nein ( )

- Haben Sie hohen Blutdruck? Ja ( ) nein ( )

- Nehmen Sie herzfrequenzsenkende Medikamente?

- Nehmen Sie blutdrucksenkende Medikamente?

z.B. Betablocker? Ja ( ) Nein ( )

Welche $? . \ldots \ldots \ldots \ldots \ldots$. . .

- Medikamente?................

- Bewegungseinschränkungen Ja ( ) nein ( ) wenn ja

Welche?

- Psychotherapie Behandlung Ja ( ) nein ( )

- Sind Sie schon mal stationär wegen Ihres Übergewichts behandelt worden? Ja ( ) nein ( )

Meines Wissens bestehen keine medizinische Einschränkung der körperlichen Belastbarkeit

Unterschrift: 


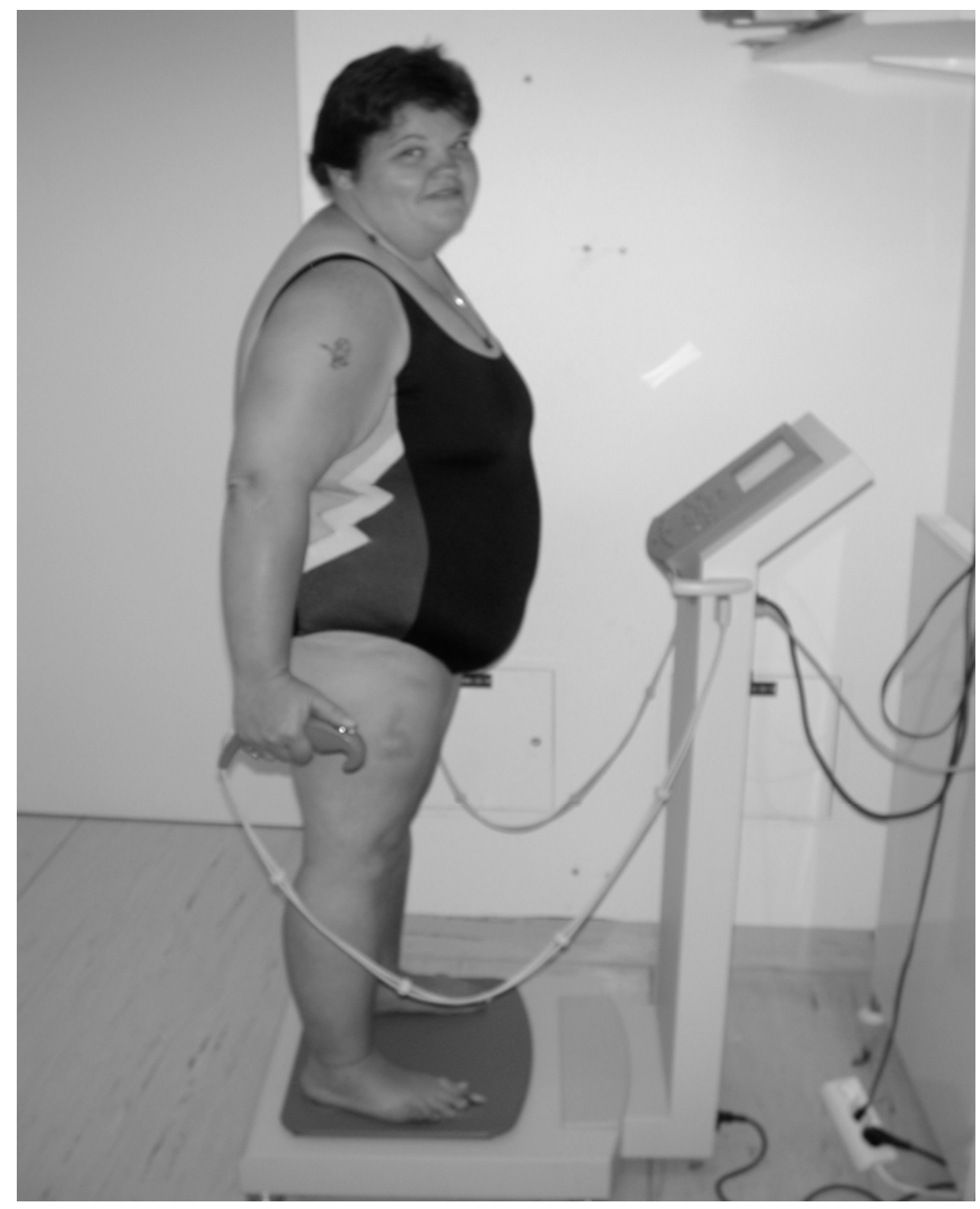

Bild: Körperzusammensetzungsbestimmung mit InBody 3.0 


\section{Fragebogen zur Erfassung der sportlich- körperlichen Aktivität}

\section{Ihre sportliche Aktivität}

1) Treiben Sie Sport?

ja [1] nein [0] Wann haben Sie damit angefangen?

2) Wie intensiv ist Ihre sportliche Aktivität dabei in der Regel?

locker und leicht (ohne Schwitzen oder Kurzatmigkeit)

flott und zügig (etwas Schwitzen und Kurzatmigkeit)

hart und angestrengt (deutliches Schwitzen und Kurzatmigkeit)

3) Wie oft sind Sie in der Regel sportlich aktiv?

weniger als einmal pro Woche

einmal pro Woche

zweimal pro Woche

dreimal oder öfter pro Woche

4) Wie lange dauern Ihre Übungseinheiten in der Regel? (Bitte berücksichtigen Sie nur die reine Übungszeit ohne Transportwege, Duschen, ...)

weniger als 20 Minuten

20 bis 40 Minuten

mehr als 40 Minuten

5) Welche Sportarten betreiben Sie?

\begin{tabular}{|ll|l|}
\hline Sportart & Min./Woche & $\mathrm{Km} /$ Woche \\
Joggen & & \\
Schwimmen & & \\
\cline { 2 - 3 } Radfahren & \multicolumn{1}{l}{} \\
\hline Skilanglauf (im Winter) & Min./Woche & \\
\hline Andere Sportarten: (Bitte eintragen!) & & \\
\hline
\end{tabular}

6) Sind Sie Mitglied in einem Sportverein oder einem anderen Sportanbieter (Kirche, Betriebssport, Selbsthilfegruppe)?

7)Treiben Sie außerhalb Organisationen oder Vereine Sport ? :

8) Haben Sie seit Ihrer Schulzeit durchgehend Sport betrieben? (keine Pausen, die länger als ein Jahr dauerten)

ja

nein [0] 
Ihr sportlicher Werdegang

9) Blicken Sie bitte zurück! Haben Sie vor 10 Jahren Sport betrieben?
ja [1]
nein [0]

Wenn "ja", vergleichen Sie bitte das Ausmaß Ihrer damaligen sportlichen Aktivität mit heute: Haben Sie vor 10 Jahren weniger [1] genauso viel [2] mehr [3] Sport betrieben als heute?

10) Blicken Sie in die Zukunft! Glauben Sie, daß Sie in 10 Jahren Sport treiben werden?

ja

nein [0]

Wenn "ja", glauben Sie, daß Sie in 10 Jahren weniger [1] genauso viel [2] mehr [3] Sport treiben werden als heute?

\section{Ihre körperliche Aktivität außer beim Sport}

11) Wie groß ist die Entfernung, die Sie an einem typischen Wochentag zu Fuß gehen? (Berücksichtigen Sie dabei alle Strecken auf dem Weg zur Arbeit, während der Arbeit und in der Freizeit.)

ich gehe fast nie zu Fuß

[1]

weniger als $1 \mathrm{~km} / \mathrm{Tag}$ (nur im Haus)

1-2 km/Tag (im Haus und kleinere Gehstrecken)

3-5 km/Tag (größere Gehstrecken außer Haus)

6-9 km/Tag

$10 \mathrm{~km}$ und mehr [6]

12) Benutzen Sie das Fahrrad - wenn das Wetter es zuläßt - täglich oder fast täglich zur Fortbewegung (zur Arbeit, zum Einkaufen etc.) an einem typischen Wochentag?

nein .................. [0 0 ja

[1] und meine tägliche Fahrradstrecke beträgt ca. $\mathrm{km}$.

13) Führen Sie andere körperlich anstrengende Freizeittätigkeiten fast täglich aus? (z.B. Gartenarbeit) ja [1] nein [0]

Falls ja, welche?

Wieviel Zeit verbringen Sie insgesamt damit (außer Sport)? Min./Woche

14) Wie würden Sie Ihre Tätigkeit am Arbeitsplatz, oder falls Sie Hausfrau/mann sind, ihre Hausarbeit, beschreiben? (Kreuzen Sie bitte die für Sie zutreffende Aussage an)

Ich arbeite überwiegend sitzend.

Ich arbeite überwiegend stehend.

Ich bin bei der Arbeit überwiegend in Bewegung.

15) Wieviel körperliche Anstrengung erfordert diese Tätigkeit?

keine besondere körperliche Anstrengung

mäßige körperliche Anstrengung

schwere körperliche Anstrengung 


\section{Der Dortmunder Fragebogen zur Bewegungstherapie (DFBT)}

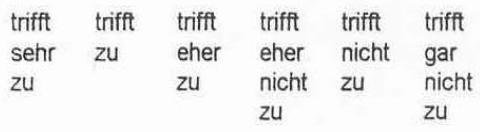

1. In dem Kurs konnte ich negative bzw. positive Gefühle offen gegenüber anderen Gruppenmitgliedern äußern.

2. In dem Kurs gehörte ich zu einer Gruppe von Menschen, die mich verstand und akzeptierte.

3. Durch den Kurs sind meine körperlichen Empfindungen vielfältiger und intensiver geworden.

4. In dem Kurs habe ich darüber sprechen können, was mich innerlich bewegt.

5. Durch den Kurs habe ich besser verstanden, wie ich in meiner Familie aufgewachsen bin.

6. In dem Kurs habe ich erfahren, wie ich mehr auf meinen Körper achte.

7. Durch den Kurs konnte ich mich selbst wieder besser "in Griff" bekommen.

8. Durch den Kurs habe ich erfahren, warum ich so und nicht anders denke und fühle.

9. Durch den Kurs habe ich erfahren, dass mein heutiges Fühlen und Verhalten mit meiner Kindheit und meiner Entwicklung zusammen hängt.

10. In dem Kurs konnte ich negative bzw. positive Gefühle offen gegenüber der/dem Therapeutin/ Therapeuten äußern.

11. In dem Kurs war die Gruppenatmosphäre für mich angenehm.

12. In dem Kurs habe ich gelernt, wie ich meine Bewegungen und meine Haltung deutlicher spüren kann.

13. Durch den Kurs kann ich die Vorgänge in meinem Körper jetzt besser verstehen. 
14. In dem Kurs habe ich mich als Gruppenmitglied wie in einer Familie verstanden und akzeptiert gefühlt.

15. Durch den Kurs kann ich mich jetzt besser entspannen.

16. Durch den Kurs habe ich die Spannungen besser zu verstehen gelernt, die ich in der Vergangenheit mit meinen Eltern,

Geschwistern und anderen wichtigen Personen hatte.

17. In dem Kurs habe ich erfahren, dass ich mich Menschen und Situationen gegenüber mit solchen Gefühlen verhalte, wie ich sie von früher kenne.

18. In dem Kurs erlebte ich irgendwie die Familie, in der ich aufwuchs.

19. In dem Kurs habe ich erlebt, dass mir die Bewegung einfach gut tut.

20. In dem Kurs konnte ich aktiv etwas für meine Gesundheit tun.

21. In dem Kurs habe ich erlebt, dass ich mich nach körperlicher Anstrengung wohlfühle.

22. In dem Kurs konnte ich die körperliche Bewegung einfach genießen. 


\section{Exemplarische Darstellung einer Therapieeinheit (90 min) des psychoedukativen Bewegungsprogramms für Männer und Frauen mit Adipositas}

Therapieziele: Körperkontakt, Körperberührung, Nähe und Distanz

Interventionsmerkmal: Sporttherapeutische und psychomotorische Elemente

\begin{tabular}{|c|c|c|c|}
\hline Phase & Inhalte & Methode/Organisationsform & Ziele \\
\hline Ausklang & $\begin{array}{l}\text { Kleine Spiele } \\
\text { (Kraft betonend wie „Esel treiben“) } \\
\text { „Kampfübungen“ und Kraftübungen } \\
\text { (z.B. Armdrücken) } \\
\text { Abklopfen und Massage verschiedener } \\
\text { Körperteile } \\
\text { Igelballmassage der Rückseite des } \\
\text { Körpers } \\
\text { Rückenlage, „Im eigenen Raum liegen“ } \\
\text { Entspannungsübungen mit Musik }\end{array}$ & $\begin{array}{l}\text { Partnerarbeit im Stehen } \\
\text { (wenn nötig im Sitzen) } \\
\text { ، “ “ “ “ “ } \\
\text { ، “ } \\
\text { Partnerarbeit im Liegen } \\
\text { Unterstützende und beratende } \\
\text { Funktion des Therapeuten } \\
\text { Entspannungsübungen in Ruhe } \\
\text { Einsatz von Musik } \\
\text { unterstützende und beratende } \\
\text { Funktion des Therapeuten }\end{array}$ & $\begin{array}{l}\text { Erleben der Körperspannung } \\
\text { Angenehmes, wertfreies } \\
\text { Berühren und Sich-Berühren-Lassen } \\
\text { körperliche Nähe in einem } \\
\text { sportlich-bewegungstherapeutischen } \\
\text { Kontext spüren, persönliche } \\
\text { Reflexion über Nähe als } \\
\text { Grundbedürfnis }\end{array}$ \\
\hline
\end{tabular}


Exemplarische Darstellung einer Therapieeinheit des psychoedukativen Bewegungsprogramms (90 min) für Männer und Frauen mit Adipositas

Therapieziele: Wahrnehmung des Körpers im Wasser, Verbesserung der körperlichen Leistungsfähigkeit, Informationsvermittlung, Motivationssteigerung

Interventionsmerkmal: Sporttherapie, Psychomotorik im Wasser, Kognitive Therapie

Sporttherapeutische Einheit im Wasser (60 min)

\begin{tabular}{|c|c|c|c|}
\hline Phase & Inhalte & Methode/Organisationsform & Ziele \\
\hline Einstimmung & $\begin{array}{l}\text { verschiedenen Bewegungsformen des } \\
\text { Aquajoggings (Laufen) }\end{array}$ & $\begin{array}{l}\text { Gruppenmitglieder laufen nebeneinander, } \\
\text { Einsatz von Musik } \\
\text { Anleitung durch den Therapeuten }\end{array}$ & psychisches und physisches Aufwärmen \\
\hline Hauptteil & $\begin{array}{l}\text { Wasserball spielen } \\
\text { Fahrrad fahren am Beckenrand } \\
\text { Füße und Beine unter Wasser massieren }\end{array}$ & $\begin{array}{l}2 \text { Gruppen } \\
\text { Einzelarbeit } \\
\text { “ “ “ " “ } \\
\text { unterstützende und beratende Funktion des } \\
\text { Therapeuten }\end{array}$ & $\begin{array}{l}\text { Freude an Spiel mit anderen gewinnen } \\
\text { Förderung der Ausdauerfähigkeit }\end{array}$ \\
\hline Ausklang & $\begin{array}{l}\text { „blind“ mit der Hilfe des sehenden Partners } \\
\text { im Wasser gehen } \\
\text { „blind“ allein im Wasser gehen, stehen } \\
\text { bleiben bzw. hinlegen u. vom Wasser } \\
\text { getragen werden }\end{array}$ & $\begin{array}{l}\text { paarweise } \\
\text { Entspannungsübungen in Ruhe } \\
\text { unterstützende und beratende Funktion des } \\
\text { Therapeuten }\end{array}$ & $\begin{array}{l}\text { das Gefühl des „Getragenwerdens“ erleben } \\
\text { Spannungsregulation, Ausklang } \\
\text { “ “ “ “ “ }\end{array}$ \\
\hline
\end{tabular}

Psychoedukative Einheit im Anschluss an die wassertherapeutische Einheit (30 min)

\begin{tabular}{|c|c|c|}
\hline $\begin{array}{l}\text { „Auswahl der Sportarten bei Adipositas“ } \\
\text { „Bewegungstagebuch, }\end{array}$ & $\begin{array}{l}\text { Vortrag } \\
\text { Gruppendiskussion- Austausch } \\
\text { Gruppe sitzt in Kreisform }\end{array}$ & $\begin{array}{l}\text { Wissensvermittlung } \\
\text { Erfahrung von sozialer Unterstützung }\end{array}$ \\
\hline
\end{tabular}


Ambulantes Adipositas Bewegungsprogramm Jannis Alexandridis

Klinik Roseneck

\title{
Prien
}

Arbeitsblätter

\author{
zum \\ ambulanten
}

Bewegungsprogramm

für Männer und Frauen

mit starkem

Übergewicht

Name:. ........ 


\section{$\underline{\text { Warum Bewegung/Fitness? }}$}

Übergewicht entsteht meistens aus dem Ungleichgewicht zwischen

Nahrungsaufnahme (Kalorienzufuhr) und Energieausgabe

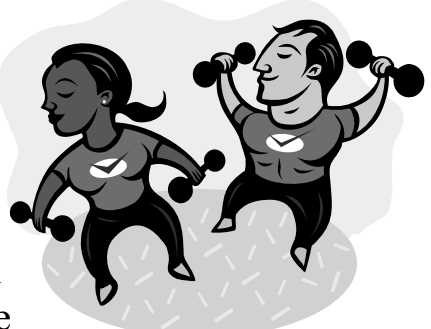

(Kalorienverbrauch). So wie wir leben, gibt es auf der einen Seite ein uneingeschränktes Angebot an Nahrungsmitteln und auf der anderen Seite ist körperliche Bewegung immer weniger nötig. Wer ständig mehr isst als er verbraucht, setzt dauerhaft Fettpolster an.

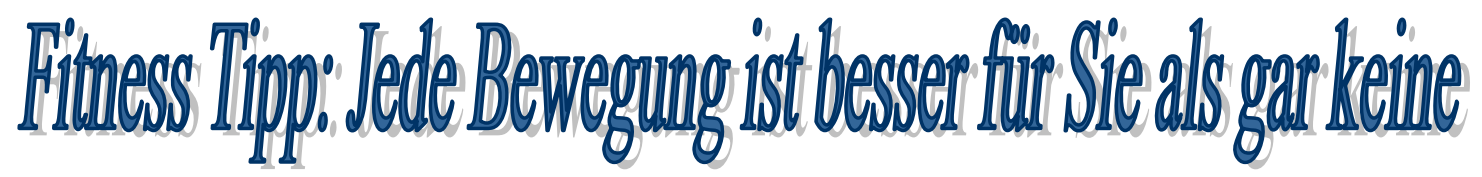

Was versteht man unter Bewegung?

Bewegung kann eine sportliche Aktivität sein, wie 1 Stunde Tischtennis spielen, aber genauso jede alltägliche körperliche Handlung wie Haushalt, Gartenarbeit, mit dem Hund spaziergehen, tanzen gehen etc.. -Bewegung ist Leben und Leben ist Bewegung

\section{Bewegung verbessert die Fitness.}

\section{Je aktiver Sie werden, desto mehr gewinnen Sie an Fitness}

\section{Positive Effekte einer verbesserten Fitness sind:}

$>$ gesündere Blutwerte, Blutdruck etc..

$>$ schnellere Gewichtsabnahme

$>$ Sie halten nach Ende der Therapie Ihr Gewicht im „grünen Bereich“

$>$ eine bessere und positivere Einstellung zu sich selbst zu bekommen.

$>$ Das Wohlbefinden steigt.

$>$ weniger Depressionen und Ängste.

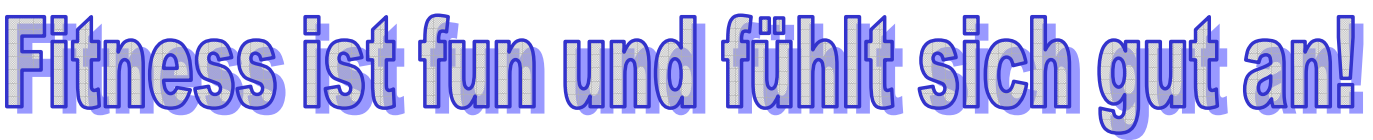


Welcher Sport-Sportart ist für mich qeeignet?

FITNESS TIPP: DIE IDEALE SPORTART IST IMMER DIE, DIE IHNEN AM MEISTEN

SPAß MACHT UND ZU IHNEN PASST...

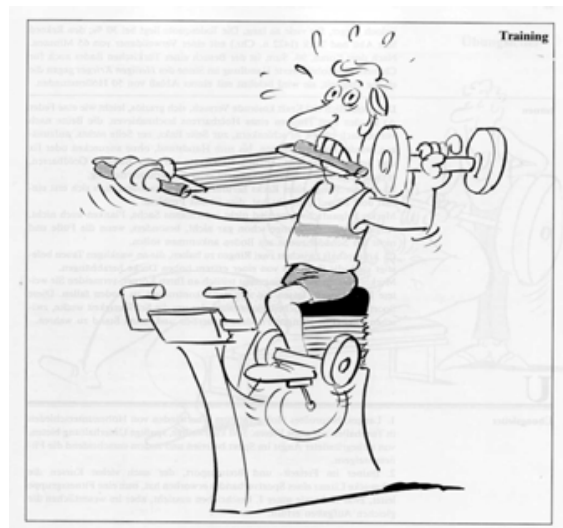

Nicht jeder von uns hat die gleiche Veranlagung und die gleichen Interessen auch bei Sport nicht, deshalb finden Sie „Ihre Bewegung“, die Ihnen Spaß macht, denn:

SPAB IST DIE HALBE MIETE

Welche ist Ihre körperliche oder sportliche Lieblingsaktivität?

Welche Sportarten sind geeignet?

Als besonders geeignet für übergewichtige Menschen sind Sportarten die gelenkschonend und rückenschonend den ganzen Körper fordern, z. B. Walken, Radeln, Schwimmen, Gymnastik.

Wichtig für Ihre Kondition und körperliche Fitness ist eine Kombination aus Beweglichkeit, Ausdauer und Kraftübungen und ein Wechselspiel verschiedener Sportarten.

Achten Sie auf Abwechslung 


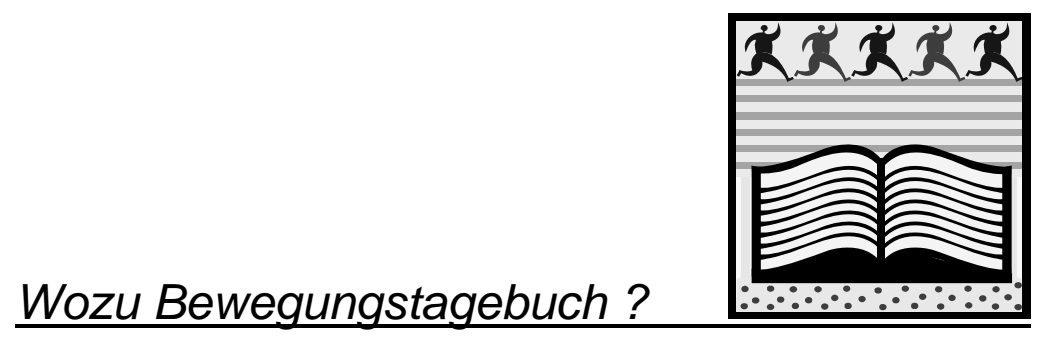

\author{
FITNESS TIPP: DAS BEWEGUNGSTAGEBUCH SOLL IHNEN \\ HELFEN ÜBER IHRE KÖRPERLICHEN AKTIVITÄTEN (BEWUSST) \\ NACHZUDENKEN.
}

\title{
Wie aktiv sind Sie ?
}

\section{Wie aktiv wollen Sie sein?}

Mit der Hilfe eines Wochenplans können Sie die Zeit und die Art ihrer täglichen körperlichen und sportlichen Aktivitäten aufschreiben. Seien Sie genau!

Das Bewegungsbuch dient zunächst dazu eine Eigenkontrolle über Ihre körperlichen Aktivitäten zu haben. Weiterhin sollen Sie dadurch die Hindernisse identifizieren, die sie von Ihren körperlichen Aktivitäten abhalten.

Durch das Bewegungstagebuch können Sie Ihre Fortschritte messen.

Das Bewegungstagebuch hilft Ihnen sich Ihre persönlichen Ziele für die kommende Woche zu setzen.

Beschreiben Sie Ihre Ziele zeitlich so genau wie möglich. 
Wie sehen die Bewegungen in Ihrem Alltag aus?

Beispiel: Durchschnittlicher Bewegungsalltag von Büroangestellten

\section{Eln extremer bewegungsarmer Tag}

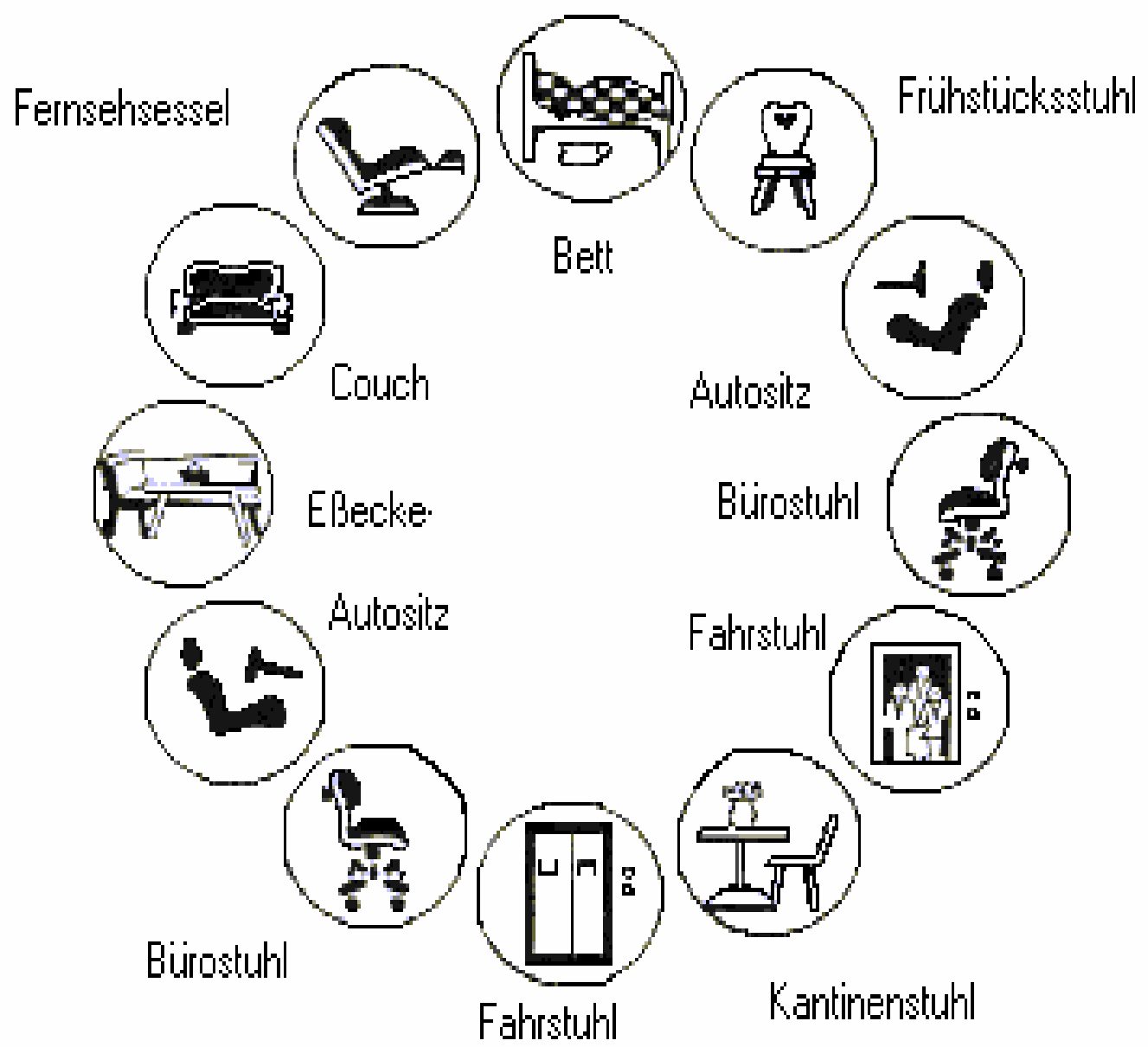

Wie sieht Ihr Tag aus?

Haben Sie und möchten Sie weiterhin Bewegung in diesen Kreis reinbringen? 
- Nach dem Essen spazieren gehen statt hinsetzen

- Treppen statt Fahrstuhl benutzen

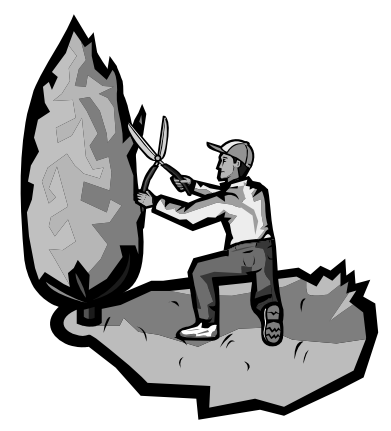

- Mit dem Fahrrad statt mit dem Auto einkaufen oder zur Arbeit fahren

- Kurze Strecken mit dem Fahrrad statt mit dem Auto fahren

- Das Auto 2 Blocks früher parken und das letzte Stück zu Fuß gehen

- Mit öffentlichen Verkehrsmitteln zur Arbeit fahren

- Steigen Sie aus dem Bus eine Haltestelle früher aus und gehen Sie das letzte Stück zu Fuß

- Mit Freunden öfter tanzen oder schwimmen statt Kino- oder Kneipenbesuche

- Stadtbummel statt Kaffeeklatsch

- Falls sie einen Hund haben, gehen Sie oft mit ihm spazieren

- Gehen Sie beim Telefonieren

- Benutzen Sie die Werbepausen im Fernsehen, um aufzustehen. Gehen Sie ins Freie und schnappen Sie frische Luft

- Aktives Hobby auswählen, z.B. Gartenarbeit, Drachen steigen lassen, schwimmen statt lesen und Fernsehen/Video, PC-Spielen 


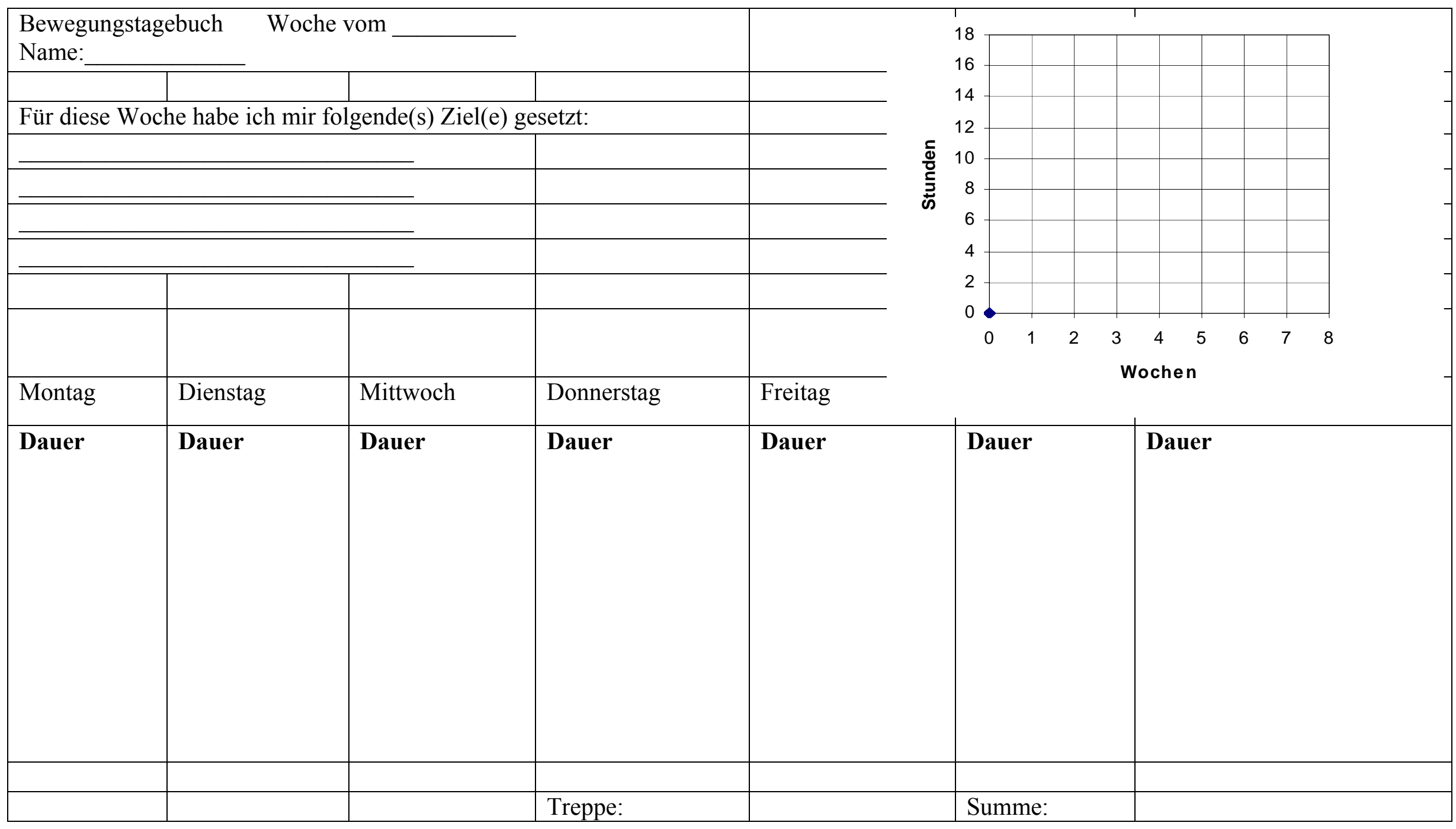

